\title{
STUDIES TOWARDS THE SYNTHESIS OF PELORUSIDE A
}

by

Bridget Louise Stocker

VICTORIA UNIVERSITY OF WELLINGTON

Te Whare Wananga o te Upoko o te Ika a Maui



A thesis

submitted to the Victoria University of Wellington in fulfilment of the

requirements for the degree of

Doctor of Philosophy in Chemistry

Victoria University of Wellington

2004 


\section{Abstract}

In the search for new treatments for cancer, advances in biology have provided targets for the destruction of cancer cells. One such structure the microtubule, a protein required for cell division, has been the target of many successful anticancer agents including the multi-million dollar earning Taxol® (paclitaxel) and the epothilones, currently in late-stage clinical trials. More recently it has been shown that peloruside A $\mathbf{1}$, a secondary metabolite isolated from the New Zealand marine sponge Mycale hentscheli, prevents cell division by stabilising microtubules, and thus offers promise as a novel anticancer agent. However, due to its limited natural abundance, significant quantities of peloruside A can only be obtained through chemical synthesis.



1

A retrosynthetic analysis of peloruside A divided the molecule into four key fragments:

a) the commercially available $\mathrm{C}-1$ to $\mathrm{C}-2$ benzyloxy acetic acid fragment;

b) the C-3 to C-7 fragment;

c) the C-8 to C-11 fragment and

d) the remaining C-12 to C-24 portion of the macrocycle and side chain.

The C-3 to C-7 and C-8 to C-11 fragments combine to form a key intermediate pyranose ring. This thesis however, addresses the synthesis of two of these key fragments, namely the $\mathrm{C}-8$ to $\mathrm{C}-11$ and $\mathrm{C}-12$ to $\mathrm{C}-24$ fragments. 
An efficient synthesis of the C-8 to C-11 fragment of peloruside A, starting from commercially available pantolactone, has been developed. This synthesis proceeds in good overall yield, and has been successfully reproduced on the multigram scale. The significant portion of this thesis, however, is dedicated to the synthesis of the C-12 to C24 fragment. After our initial strategy proved unviable, a short, facile method for the synthesis of the C-12 to C-24 fragment, involving the formation of a bis-silyl ether, was developed. The protocol for its desired coupling, via a boron-mediated, remote 1,5-antiinduction aldol reaction has also been established. These and subsequent studies provided valuable insight into the origin of 1,5-anti induction in boron-mediated aldol reactions. 
For those departed, and for those who remain. And in particular, for Darren.

Take off my shield And take my hand And with arms outstretched Expose my body and mind I give these to you freely

Then set me free

Take away my tears And dance through my soul Until only the dreams remain And I can believe

My angel 


\section{Acknowledgements}

$\mathrm{A} \mathrm{PhD} \mathrm{is} \mathrm{a} \mathrm{journey.} \mathrm{Not} \mathrm{only} \mathrm{of} \mathrm{scientific} \mathrm{discovery,} \mathrm{but} \mathrm{more} \mathrm{importantly,} \mathrm{of} \mathrm{personal}$ growth. It is interesting to look back and think about where I have come from, and where I am going, and to think about all of the people that have shaped my experience. If there is one thing that I am certain about, it's that I've certainly changed a lot over the last few years, and for this I thank the following people.

First I must thank my supervisor John. Thank you for believing in me, and thank you for your honesty and for giving me the freedom to do essentially what I like. And thank you for your friendship. Hopefully we'll meet up again some day. Equally importantly, I have to thank my Mum for teaching me the importance of determination, perseverance and self-integrity, and for always having the time to listen to - even if it is at lam in the morning. I also have to thank Egg, for offering me all of the support that only a big brother can offer.

I must also thank Peter. Thank you for your help in determining the relative stereochemistry of our key peloruside fragment, for your guidance during my dabble at natural products chemistry, and for your unique perceptiveness. And Paul. Thank you for the modelling studies, for your cheerfulness and for your willingness to always offer help. And of course I must thank Andy. Thank you for always keeping an eye on our group, and for you (stupidly) offering to read my thesis, and for doing such a fantastic job - it was a "facile" process! And then there is Prof Spencer. Thank you for being a fantastic "second" supervisor, for listening so thoughtfully, and for simply being on the first floor and peering into our lab.

And of course, during my time here, I have made three fantastic friends - forever. So thank you Sylvia. I wouldn't have shared all that time alone, in a closed room, with anybody else but you! Thank you for your amazing free spirit. And Shivali. Thank you for always finding the time to talk, especially during those "second year blues". I'll never 
have another friend quite like you! And Anna. Thank you for being my social organising committee and for never failing to encourage me to have a life. Even though you are the youngest, thank you for being the mother hen to all of us.

And then there are my other good friends that have always been there for me. So thank you Paul. Your honesty and openness is incredible. Thank you for always making me see the best in people. You will always be an inspiration to me. A one in a million friend. And Tim. Even though we don't see a lot of each other these days, it is reassuring to know that you are always there if I need to talk. You define the meaning of having a good time. Thank you for your brightness. And Pete. Although we parted along the way, you will always be really special to me. You really are a kind and caring person. Thank you for everything you've done for me over the years and for your unconditional support.

And of course, there is the rest of the lab group, students and staff around here, past and present. So thank you Carissa, Lyndon, Rhys, Laine, Ben, Darren, Ying, James, Wayne, Corin, Maria, Steve, Rob, John, John, Kirstin, Andy, Mike, Thomas, Ray, Jo, Oleg, Teressa, Jackie, Dave, Allan, Dave, Grant, Rhyl, Jenny, Dr Pearce, Prof Halton, Dr Weatherburn, Rob and anybody else I have forgotten. Thank you all for your uniqueness and for making a difference to my time here. And special mention must go Graham, for being my technical support guy and friend, and Simon, for disrupting us in the graduate room! (And for your friendship also). And of course I must thank Sally. Your resilience is amazing. Thank you for bringing some life into the department and for the chats. And thank you Bill, for favouring Sylvia over me!

And of course I have to thank Lyndsey, Christine, Mick and John, and especially Stevie, for teaching me the importance of growth and forgiveness and for reminding me how to dream.

"When the rain washes you clean ... you'll know"

S. Nicks, CA 2003 


\section{Table of Contents}



Preface 1

\section{Chapter 1: Introduction}

1.1: Tubulin stabilising antimitotic compounds - an overview ...................... 2



1.3: Tubulin binding agents and cell death

1.3.1: The two divisions of antimitotic compounds ......................... 9

1.3.2: Mode of action of the antimitotic agents ............................. 13

1.4: Biochemistry of peloruside $\mathrm{A}$



1.4.2: Cytotoxicity and the identification of apoptotic pathways ............... 16







1.8: Determination of the absolute stereochemistry of peloruside A $\ldots \ldots \ldots \ldots \ldots \ldots .22$ 
1.9: Synthetic strategies towards the synthesis of peloruside A



1.9.2: Synthesis of the C-3 to C-11 fragment of peloruside A ................. 24

1.9.3: Synthesis of the C-12 to C-24 fragment of peloruside A ............... 28



\section{Chapter 2: Determination of the absolute stereochemistry of peloruside A}







\section{Chapter 3: Synthesis of the C-8 to C-11 fragment of peloruside A}





\section{Chapter 4: Synthesis of the C-12 to C-24 fragment of peloruside A: Strategy 1:}

4.1: The lactone approach

\section{Chapter 5: Synthesis of the C-12 to C-24 fragment of peloruside A: Strategy 2:}

5.1: Initial aldol studies with the cyclic bis-silyl ethers

5.2: Adaptation to a more linear approach for the synthesis of the C-12 to C-24 fragment of peloruside A

5.3: $\quad$ Revisiting the original cyclic bis-silyl ether strategy ......................92



\section{Chapter 6: Asymmetric synthesis of the $\mathrm{C}-12$ to $\mathrm{C}-24$ fragment of peloruside $A$}

6.1: Key stereochemical features 
6.2: Synthesis of (R)-4-hydroxy-5-methylhex-5-en-2-one

6.2.1: The kinetic resolution of ( \pm )-4-hydroxy-5-methylhex-5-en-2-one .99

6.2.2: Asymmetric aldol reactions

6.3: Boron mediated 1,5-anti aldol reactions

6.3.1: A brief overview 107

6.3.2: The proposed transition state for the boron mediated 1,5-anti induction for the synthesis of peloruside $\mathrm{A}$ 110

6.3.3: Studies into the synthesis of peloruside A. 111

6.3.4: Determination of the anti stereochemistry.... 116

6.4: Putting the pieces of the puzzle together 121

\section{Chapter 7: Studies into the origin of 1,5-anti induction in boron-mediated aldols}

7.1: Acyclic systems

7.1.1: The tert-butyl diphenyl silyl derivative 124

7.1.2: The dimethylphenyl silyl derivative

7.1.3: Benzyl as a protecting group 128

7.1.4: Conclusions 130

7.2: Cyclic systems

7.2.1: The pyranose system.

7.3: Boron mediated 1,5-anti induction - a summary

Chapter 8: Concluding Remarks .139

\section{Chapter 9: Experimental Section}

























\section{List of Figures}

\section{Chapter One}

1. Polymerisation of tubulin to microtubules.................................. 6

2. Dynamic equilibria of tubulin microtubules................................... 6



4. Blocking of mitotic spindle division by antimitotic compounds............... 9

5. Selected natural products promoting tubulin depolymerisation $\ldots \ldots \ldots \ldots \ldots \ldots \ldots \ldots \ldots \ldots \ldots \ldots \ldots$

6. Selected natural products with tubulin polymerisation and microtubule stabilisation properties..................................................... 12

7. TEM of pel A induced polymerised tubulin................................. 15

8. Western blotting showing the effects of Taxol, peloruside A and colchicine



9. H441 Human lung adrenocarcinoma cells showing cytoskeletal bundles.........16





\section{Chapter Two}

12. Gas chromatograph trace of the racemic aldehyde $44 \mathrm{~b}$ co-injected with the $(S)$-enantiomer 44

13. Model substrates for small-scale ozonolysis reactions

\section{Chapter Three}

14. ${ }^{1} \mathrm{H}$ NMR of gem dimethyl ketone 8 . 60

\section{Chapter Four}

15. Examples of common metal-alkylidine metathesis catalysts .62

16. ${ }^{13} \mathrm{C}$ NMR of lactone $\mathbf{5 6 b}$ 


\section{Chapter Five}

17. ${ }^{\mathrm{I}} \mathrm{H}$ NMR spectrum of 4,8-O-diphenylsilanediyl-5-methyloct-5-en-2-one $\mathbf{6 5 a} . \ldots . .82$

18. 4,8-O-diphenylsilanediyl-5-methyloct-5-en-2-one 65a $\ldots \ldots \ldots \ldots \ldots \ldots \ldots \ldots \ldots \ldots \ldots \ldots \ldots \ldots \ldots$



\section{Chapter Six}

20. Structural similarities between peloruside A and epothilone B................ 97

21. Classic versus Dynamic Kinetic Resolution.............................. 100

22. GC trace of the silylated enantiomers ...................................... 106

23. Examples of boron mediated 1,5-anti aldol reactions........................ 108

24. $\pi$ stacking effects in asymmetric synthesis................................. 109

25. ${ }^{13} \mathrm{C}$ NMR of a diastereomeric mixture of 7,11-O-diisopropylsilanediyl -2,2-dimethyl-3-hydroxyundec-8-en-5-one.

26. ${ }^{13} \mathrm{C} \mathrm{NMR}$ of a diastereomeric mixture of 7,11-O-diphenylsilanediyl-2,2dimethyl-3-hydroxyundec-8-en-5-one.

27. ${ }^{1} \mathrm{H}$ NMR of $(3 R, 7 R)$-7,11- $O$-diphenylsilanediyl-2,2,8-trimethylundec-8-en5-one.

28. ${ }^{13} \mathrm{C}$ NMR of (3R,7R)-7,11-O-diphenylsilanediyl-2,2,8-trimethylundec-8-en5-one.

29. NOe correlations of $(3 R, 7 R)$-7,11-O-diphenylsilanediyl-3-hydroxy-2,2,8-trimethylundec-8-en-5-one.

30. Molecular modelling of ${ }^{\mathrm{i}} \mathrm{Pr}_{2} \mathrm{Si}$ and $\mathrm{Ph}_{2} \mathrm{Si}$ enol ethers.

120

\section{Chapter Seven}

31. Possible conformations for the tert-butyldiphenylsilyl boron enol ether 125

32. Proposed transition state for the boron enolate of $\mathbf{7 9}$....

33. Molecular modelling of the methyl enol ether of pyranose $\mathbf{1 0 3}$ 133

34. Proposed transition state for the boron-mediated aldol reaction 


\section{Concluding remarks}

35. Peloruside A with the C-8 to C-24 framework highlighted................... 139 


\section{List of Schemes}

\section{Chapter One}

1. Methodology for the determination of the absolute stereochemistry of peloruside $\mathrm{A}$





4. Control of the stereoselectivity of the pyranose ring by B-alkoxy lithium chelation

5. Alternative strategy for the synthesis of the pyranose ring.

6. Synthesis of ketone $\mathbf{3 8}$ starting with glyoxal and isobutyrlaldehyde.

7. Synthesis of ketone 38 starting with pantolactone................................



9. Initial retrosynthetic analysis for the formation of the C-12 to C-24 fragment....28







\section{Chapter Two}

13. Strategy for the determination of the absolute stereochemistry of peloruside A ..33

14. Formation of tert-butyldimethylsiloxypropanal............................. 34



16. Attempted synthesis of $N$-cyclohexyl-3-(tert-butyldimethysiloxy)imine $\ldots \ldots \ldots 35$

17. Alternative strategy for the racemic synthesis of the fragment $\mathbf{3 b} \ldots \ldots \ldots \ldots \ldots \ldots$

18. Literature precedent for the synthesis of (-)-(2S)-2-((tert-butyldimethylsiloxy)-



19. Final oxidation of the enantiomerically pure alcohol......................... 37

20. The attempted silylation of peloruside A................................... 38




22. Alternative strategy for the synthesis of an enantiomerically pure side chain $\mathbf{4 4 . . 4 0}$

23. Formation of the acetylated racemic side chain $44 \mathrm{~b}$ 41

24. Synthesis of the acetylated, enantiomerically enriched side chain $44 \ldots \ldots \ldots \ldots . . \ldots 2$



26. Attempted ozonolysis of acetylated peloruside A.......................... 46

27. Swern oxidation of alcohol 39b......................................... 47



29. Model studies for the oxidative cleavage of peloruside A....................48

30. Attempted osmate/periodate olefinic cleavage of peloruside A.................48

\section{Chapter Three}





33. Modified strategy for the formation of gem-dimethylketone $8 \ldots \ldots \ldots \ldots \ldots \ldots \ldots 1$

34. Protection of pantolactone 17 and attempted reduction .......................5 52















\section{Chapter Four}

42. Retrosynthetic analysis for the synthesis of the C-12 to C-24 fragment....

43. Complete retrosynthetic analysis for the synthesis of the model $\mathrm{C}-12$ to C-24 fragment

44. Formation of B-hydroxyketones 24a and 24b 65

45. Synthesis of esters $\mathbf{5 7} \mathbf{a}$ and $\mathbf{5 7} \mathbf{b}$ 






48. Metathesis reactions of esters $57 \mathbf{b}$ using first generation Grubbs' catalyst........69

49. Proposed pathways for catalyst generation and decomposition....................70



51. Model aldol studies with lactones 56a and 56b......................... 73

52. Alternative strategy for the synthesis of the C-12 to C-24 fragment of peloruside A.

\section{Chapter Five}

53. Retrosynthetic strategy (II) for the synthesis of the C-12 to C-24 fragment



54. Simplified retrosynthetic analysis for synthesis of the C-12 to C-24 fragment.... 76

55. Synthesis of the but-3-en-1-oxydialkylsilyl chlorides ........................ 77

56. Synthesis of the but-3-en-1-oxydialkylsilyl chloride derivatives $66 \ldots \ldots \ldots \ldots \ldots 78$

57. Attempted reversal of addition of the hydroxy substrates..................... 79

58. Desired RCM of the but-3-en-1-oxydialkylsilyl chloride derivatives.............. 80

59. Model aldol reactions with the bis-silyl ethers.............................. 84





62. Attempted formation of the tert-butyl tethered ether......................... 87

63. Attempted RCM of the dialkylated di-tert-butyl silyl derivative $98 \ldots \ldots \ldots \ldots 87$

64. A more linear approach for the synthesis of peloruside A..................... 88



66. Silylation of the $B$-hydroxy ketones......................................... 89

67. Etherification of the B-hydroxy ketones...................................... 90

68. Model aldol studies with pivaldehyde...................................... 90

69. Benzylation of the aldol adducts with benzyl trichloroacetimidate.............. 91





72. Attempted synthesis of $\mathbf{6 5 b}$ by Mo-catalysed RCM........................ 94 


\section{Chapter Six}

73. Retrosynthesis of peloruside A showing the required 1,5-anti relationship between the C-11 and C-15 stereocentres .................................. 98

74. Retrosynthesis for the formation of the C-12 to C-24 fragment of peloruside A. 98

75. Mechanism of the ruthenium-catalysed hydrogen transfer reaction. 100

76. Proposed products from the in situ racemisation of the starting substrate during the attempted DKR of the B-hydroxy ketone

77. Attempted kinetic resolution of ( \pm )-4-hydroxy-5-methyl-5-hexen-2-one using either Candida antartica or Candida rugosa.

78. (L)-Proline catalysed asymmetric aldol reaction for the synthesis of

(R)-4-hydroxy-5-methylhex-5-en-2-one.

79. Proposed mechanism of the $(L)$-proline catalysed aldol reaction............... 105

80. The basic boron mediated 1,5 -anti aldol reaction ......................... 107

81. Proposed transition state for asymmetric control.........................110

82. Boron mediated 1,5-anti aldol studies.................................. 112

83. Proposed transition state for the boron mediated 1,5-anti aldol reactions



84. Proposed conformations for the acyclic phenyl derivatives...................122

\section{Chapter Seven}

85. Synthesis of the tert-butyldiphenylsilyl protected B-hydroxy ketones............ 126

86. Aldol studies with the tert-butyldiphenylsilyl derivatives................... 126

87. Synthesis of the dimethylphenylsilyl derivatives .......................... 127

88. Aldol studies with the dimethylphenylsilyl derivatives..................... 127

89. Aldol studies with the benzyl protected derivative...........................129

90. Boron mediated 1,5-anti aldol studies in cyclic systems.................... 132









95. Boron mediated 1,5-anti aldol studies conducted by De Brabander et. al........135 


\section{List of Tables}

\section{Chapter Five}

1. Yields for the formation of 3-butenoxydialkylsilyl chloride derivatives..........78

2. Synthesis of bis-silyl cyclic ethers 72 by RCM........................... 81



\section{Chapter Six}

4. Boron mediated 1,5-anti aldol studies ......................................



\section{Chapter Seven}

6. Boron mediated aldol studies with the tert-butyldiphenylsilyl derivatives....... 126

7. Boron mediated aldol studies with the dimethylphenylsilyl derivatives......... 128






\section{Glossary}

\begin{tabular}{|c|c|}
\hline $\mathrm{AcOH}$ & acetic acid \\
\hline BINOL & 1,1'-Bi(2-naphthol) \\
\hline bs & broad singlet \\
\hline $\mathrm{CDCl}_{3}$ & deuterated chloroform \\
\hline CDI & carbonyldiimidazole \\
\hline $\mathrm{CH}_{2} \mathrm{Cl}_{2}$ & dichloromethane \\
\hline COD & cyclooctadienyl \\
\hline COSY & correlation spectroscopy \\
\hline Cy & cyclohexyl \\
\hline d & doublet \\
\hline $\mathrm{DABCO}$ & 1,4-diazabicyclo[2.2.2]octane \\
\hline DIBAL-H & diisobutylaluminium hydride \\
\hline DCC & dicyclohexylcarbodiimide \\
\hline DEPT & Distortionless Enhancement by Polarisation Transfer \\
\hline DKR & Dynamic Kinetic Resolution \\
\hline DMAP & dimethylaminopyridine \\
\hline DMF & $N, N$-dimethylformamide \\
\hline DMSO & dimethylsulfoxide \\
\hline ds & diastereoselectivity \\
\hline ee & enantiomeric excess \\
\hline $\mathrm{Et}_{2} \mathrm{O}$ & diethylether \\
\hline EtOAc & ethyl acetate \\
\hline $\mathrm{E} 1_{\mathrm{cb}}$ & Elimination unimolecular conjugate base \\
\hline EDCI & 1-[3-(Dimethylamino)propyl]-3-ethyl-carbodiimide hydrochloride \\
\hline FID & Flame Ionisation Detector \\
\hline G1 & gap 1 \\
\hline G2 & gap 2 \\
\hline $\mathrm{G} 2 / \mathrm{M}$ & gap $2 /$ mitosis \\
\hline
\end{tabular}




\begin{tabular}{|c|c|}
\hline $\mathrm{GC}$ & gas chromatograpy \\
\hline GDP & guanosine 5'-diphosphate \\
\hline GTP & guanosine 5'-triphosphate \\
\hline HMPA & hexamethylphosphoramide \\
\hline HP-20S & Poly(styrene-divinylbenzene) stationary support (Supelco) \\
\hline HPLC & high pressure liquid chromatography \\
\hline HRESIMS & High Resolution Electro-spray Ionisation Mass Spectroscopy \\
\hline HSQC & Heteronuclear Single Quantum Coherence \\
\hline $\mathrm{IC}_{50}$ & $50 \%$ Inhibitory Concentration \\
\hline Ipc & isopentylcamphenyl \\
\hline IR & Infrared \\
\hline$J$ & scalar coupling constant \\
\hline $\mathrm{LiAlH}_{4}$ & lithium aluminium hydride \\
\hline $\mathrm{LiBH}_{4}$ & lithium borohydride \\
\hline Lipase $P S$ & Lipase Pseudomonas cepacia \\
\hline LDA & lithium diisopropylamide \\
\hline LTMP & lithium tetramethylpiperidide \\
\hline $\mathrm{m}$ & multiplet \\
\hline M phase & mitosis phase \\
\hline MAPs & microtubule associated proteins \\
\hline mes & mesitylene (1,3,5-trimethylbenzene) \\
\hline MDR & multiple drug resistant \\
\hline MOM & methoxymethyl ether \\
\hline MTOC & microtubule organising centre \\
\hline $\mathrm{NaBH}_{4}$ & sodium borohydride \\
\hline nOe & nuclear Overhauser effect \\
\hline NMR & nuclear magnetic resonance \\
\hline $\mathrm{P}_{\mathrm{i}}$ & orthophosphate \\
\hline PCC & pyridinium chlorochromate \\
\hline PDC & pyridinium dichromate \\
\hline pel A & peloruside $\mathrm{A}$ \\
\hline
\end{tabular}




$\begin{array}{ll}\text { P-gp } & \text { P-glycoprotein } \\ \text { PMB } & \text { para-methoxy benzyl } \\ \text { Py } & \text { pyridine } \\ \text { RCM } & \text { ring closing metathesis } \\ \mathrm{R}_{\mathrm{f}} & \text { retention factor } \\ \text { rt } & \text { room temperature } \\ \text { t } & \text { triplet } \\ \text { TES } & \text { triethylsilyl } \\ \text { TEM } & \text { Transmission Electron Micrograph } \\ \text { TEMPO } & \text { 4-methoxy-2,2,6,6-tetramethyl-piperidine-1-oxyl } \\ \text { Tf } & \text { triflate } \\ \text { TBDPS } & \text { tert-butyldiphenylsilyl } \\ \text { TBHP } & \text { tert-butylhydroperoxide } \\ \text { TBS } & \text { tert-butyldimethylsilyl } \\ \text { THF } & \text { tetrahydrofuran } \\ \text { TLC } & \text { thin layer chromatography } \\ \text { TMS } & \text { trimethylsilyl } \\ \text { Ts } & \text { tosyl } \\ \text { UV } & \text { ultra violet } \\ \text { WST1 } & \text { water soluble tetrazolium } \\ { }^{13} \mathrm{C} \text { NMH } & \text { Proton Nuclear Magnetic Resonance } \\ & \end{array}$




\section{Preface}

Cancer is a group of diseases characterised by the uncontrolled growth and spread of abnormal cells that invade and disrupt other tissues and spread to other areas of the body. If the spread is not controlled, it can result in death. Cancer is a growing public health problem whose estimated worldwide new incidences are over six million cases per year. ${ }^{1}$ In New Zealand in 1999, approximately 16,700 people were diagnosed with cancer, and about 7,600 died as a result of this invasive disease. This makes cancer the leading cause of death in New Zealand, accounting for over $27 \%$ of deaths from all causes. ${ }^{2}$

This thesis represents part of a collaborative effort towards the total synthesis of the novel anti-cancer agent, peloruside $\mathrm{A}$, with the overall view to providing a practical and efficient synthesis of this compound. Studies towards the determination of the absolute stereochemistry of peloruside A will also be discussed. However, as with many scientific journeys, results can lead in unexpected directions. During the synthesis of a key fragment of peloruside $\mathrm{A}$, the opportunity arose to investigate the origin of the selectivity in boron mediated 1,5-anti aldol reactions. These results are reported accordingly. 


\section{Chapter One}

\section{Introduction}

\section{1: Tubulin stabilising antimitotic compounds - an overview}

In the search for anticancer drugs, compounds from natural sources, such as paclitaxel, extracted in 1971 from the bark of the Pacific Yew tree ${ }^{3}$ and bryostatin, isolated from a marine bryozan, ${ }^{4}$ have displayed useful anticancer activity and proven successful in clinical trials. In fact, paclitaxel, known clinically as Taxol, is one of the most successful cancer drugs ever produced. Since 1992 it has added months to the lives of over 800,000 patients with cancers of the breast, ovary or lung - in the process accumulating over $\$ 1.5$ billion a year in revenue for Bristol-Myers Squibb. Eight years after the initial discovery of paclitaxel, it was determined that its unique anti-cancer activity can be attributed to its tubulin-binding mechanism of action. ${ }^{5}$ Taxol functions by crippling a cell's ability to divide, or undergo mitosis. The drug binds to and stabilises the rod-like structures inside the cell called microtubules that, like moving train tracks, help chromosomes arrange and split in two during cell division.

However, Taxol and its late-stage semisynthetic derivative Docetaxel (taxotere) are not miracle drugs. Clinical trials with Taxol on metastasising renal ${ }^{6}$ and colon $^{7}$ carcinomas have been disappointing. Furthermore, some cancers mutate and become resistant to Taxol. ${ }^{8,9}$ Thus even when a tumour is all but eliminated by Taxol, a few cells possessing mutant phenotypes may remain, growing into more deadly tumours. Additionally, the therapeutic index of Taxol is narrow due to mechanism-based side effects of neutropenia, peripheral neuropathy, alopecia and hypersensitivity reactions. ${ }^{10}$ Taxol is also highly hydrophobic, necessitating delivery in Cremophor, which in itself can induce cardiac arrhythmias and extensive hypersensitivity reactions. ${ }^{11}$ 
Nevertheless, the phenomenal clinical success of Taxol promoted the search for new drugs with similar mechanisms of action. Once again, mother nature offered a solution in the form of the epothilones A and B, whose isolation in the late 1980's from culture extracts of the cellulose-degrading mycobacterium Sorangium cellulosum (first found in soil collected from the banks of the Zambesi River $)^{12}$ provided an additional class of tubulin stabilising anti-cancer drugs. The epothilones exhibit superiority over paclitaxel as a killer of tumour cells, particularly with cell lines exhibiting multiple drug resistance (MDR). ${ }^{13,14}$ In some of the cytotoxicity experiments, epothilone B demonstrated a 20005000 fold higher potency than paclitaxel, a striking enough observation to awaken and stimulate the interest of many in both the academic community and the pharmaceutical industry. Consequently, the epothilones and analogues thereof, are currently undergoing advanced clinical trials.

Even though paclitaxel and the epothilones are the most well known tubulin-stabilising antimitotic drugs, a small number of other tubulin-stabilising compounds exist. Of particular interest to us is the discovery that peloruside A (pel A) (1), a novel secondary metabolite isolated from a New Zealand marine sponge, Mycale hentscheli, ${ }^{15}$ exhibits potent paclitaxel-like microtubule-stabilising activity. ${ }^{16,17}$



1

Pel A belongs to one of three classes of tubulin-stabilising compounds - namely the macrolide class which includes the epothilones (to which peloruside is structurally related) and the laulimalides, ${ }^{18}$ isolated from the marine sponge Cacospongia mycofijiensis. Other classes are a) the terpenoids, including the taxanes, 
eleutherobin/sarcodictyin ${ }^{19}$ (isolated from marine corals), and the bacterial metabolite WS9885B; ${ }^{20}$ and b) the polyhydroxylated alkatetraene lactones, including discodermolide, isolated from a Carribean sponge. ${ }^{21,22}$ Reported $\mathrm{IC}_{50}$ values for the cytotoxicity of these drugs are generally in the low nM range.

Although it is possible that pel A may not introduce anything substantially new to the antimitotic group of drugs, it is anticipated that its unique structure, and hence differing bioactivity, will offer distinct advantages when compared to the other known microtubule-stabilizing compounds. Furthermore, therapeutic anti-cancer drug cocktails comprise a mixture of drugs having unique and complementary properties, ${ }^{23,24}$ to which pel A may be a valuable constituent. Multi-drug treatment regimes applied either as a single cocktail or in a rotating sequence, may help to prevent the development of resistant subclones that arise in long-term chemotherapy.

As is the case with many natural products, obtaining a sufficient quantity of the desired compound from its natural source can be a serious limitation in drug development. Although Mycale hentscheli is endemic to New Zealand, occurring in scattered populations in calm coastal water around both the North and South Islands, pel A levels in these populations are not large, with less than one third of the individuals in a population containing detectable levels of the metabolite. ${ }^{25}$ It has been estimated that to obtain $30 \mathrm{mg}$ of peloruside, $30 \mathrm{~kg}$ of sponge will have to be collected. To date, the scarcity of pel A has prevented in-depth biological studies from being conducted. However, before an overview of our strategy for the synthesis of pel A is discussed, it will be instructive to first examine further the molecular and cellular biology of tubulin binding agents, and how pel A relates to them. 


\section{2: Tubulin, microtubules and the cytoskeleton}

Tubulin is a heterodimeric protein primarily composed of $\alpha$ - and $\beta$-tubulin subunits and represents the monomeric building blocks of microtubules. Microtubules, in turn, are one of the fundamental structural components of the cytoskeleton in all eukaryotic cells. Operating either alone, or in conjunction with other proteins to form more complex structures, microtubules serve as structural beams and conveyer belts within cells. They rigidify the cell and translocate vesicles, granules, organelles and chromosomes. Although these filamentous protein networks are usually transparent and hence often neglected in cell drawings, this "nanolevel web" plays a crucial role in the dynamic organization of the interior of living cells and its existence should not be over looked.

Structually, microtubules are regular internetworked linear polymers (protofilaments) of highly dynamic assemblies of heterodimers of $\alpha$ - and $\beta$-tubulin. When thirteen of these protofilaments are arranged parallel to a cylindrical axis they self-assemble to form microtububes of approximately $24 \mathrm{~nm}$ in diameter and up to several $\mu \mathrm{m}$ in length. The $\alpha, \beta$ heterodimer is itself very compact but can be divided into three functional domains: the amino-terminal domain containing the nucleotide-binding region, the intermediate domain containing the paclitaxel-binding site, and the carboxy-terminal domain, which probably constitutes the binding surface for motor proteins. ${ }^{26}$

Formation of microtubules proceeds by a nucleation-elongation mechanism and occurs within an area of the cell called the "aster", ${ }^{27}$ Nucleation is the initial phase of the process in which preformed heterodimers of $\alpha$ - and $\beta$-tubulin assemble in the presence of $\mathrm{Mg}^{2+}$, guanosine 5'-triphosphate (GTP) and microtubule-associated proteins (MAPs). This process is relatively slow until a short microtubule is formed, triggering the much faster elongation process. The elongation phase involves the extension of the microtubule nucleus at both ends by a reversible, non-covalent addition of tubulin heterodimers to form growing oligomers that become linear rows of tubulin beads. Although both ends can either grow or dissociate, the (+)-end, which is kinetically more 
dynamic, usually grows faster than the (-)-end, (which is less dynamic). Conversely, net shortening takes place at the (-)-end, figure 1. "Treadmilling" or "flux" occurs when both of these dynamic processes occur at once, resulting in a net flow of subunits from the plus to the minus end without a significant change in microtubule length. ${ }^{27}$ Figure 2 depicts the assembly of tubulin into microtubules and the influence that paclitaxel and the epothilones have on tubulin polymerisation.

Figure 1: Polymerisation of tubulin to microtubules ${ }^{1}$


Figure 2: Dynamic equilibria of tubulin-microtubule 
Bound GTP molecules regulate the growth and dissolution of microtubules. Each tubulin dimer carries two GTP molecules, one on the $\alpha$-subunit or N site and one in the $\beta$-subunit. However only the exchangable $\beta$ subunit appears to function as a GTPase. ${ }^{28}$ During polymerisation, the GTP molecule hydrolyses to guanosine diphosphate (GDP) and orthophosphate $\left(\mathrm{P}_{\mathrm{i}}\right)$. GTP, GDP, and $\mathrm{P}_{\mathrm{i}}$ form a "cap" on the ends of the microtubules that facilitates further growth due to their high affinity for additional tubulin subunits. ${ }^{29}$ Conversely, hydrolysis of the GTP subunit on the end of the microtubule causes shortening of the microtubule. ${ }^{30}$ While the half-life of tubulin at $37{ }^{\circ} \mathrm{C}$ is nearly a full day, that of a given microtubule may be only about 10 minutes. Accordingly, microtubules are in a constant state of flux responding to the needs of the cell. This state of stochastic switching between shortening and growing phases at the ends of the individual microtubule is called "dynamic instability" and is controlled by regulatory processes within the cell. ${ }^{29,31}$

Tubulin is a major component of the cellular apparatus known as the mitotic spindle, and hence plays a crucial role throughout mitosis - the process during cell replication in which the duplicated genetic material, in the form of chromatids, is partitioned equally between the two daughter cells. Mitotic spindle microtubules are especially dynamic, and exchange their tubulin with tubulin in the cytoplasmic pool with half-lives of less than a minute. ${ }^{32}$ A cell enters mitosis after transcending through the Gap 1 (G1), chromosome replication (S phase) and Gap 2 (G2) phases of the cell cycle (collectively known as interphase), figure 3. During prophase, the first division of mitosis, the homologous sister chromatids contract and pair within the nucleus. Outside the nucleus, two dipolar spindle-shaped arrays of microtubules are formed outward from organising centres known as centrioles.' The centrioles, or microtubule-organising centres (MTOC), act as the major site of microtubule nucleation by lowering the critical concentration of tubulin required for polymerisation and anchoring the (-)-ends of the resulting microtubules. ${ }^{33}$ Near the end of prophase the nuclear envelope breaks down, allowing the spindle microtubules to enter the nuclear area. Kinetochores (mirotubule

\footnotetext{
${ }^{\mathrm{I}}$ Centrioles consist of a ring of nine groups of three fused microtubules
} 
binding proteins) then form on either face of the centromere ${ }^{\text {II }}$ and become attached to special microtubules called kinetochore microtubules. These microtubules radiate in opposite directions from each side of every chromosome and interact with the (+)-end of the spindle microtubules. Once the spindle microtubule/kinetochore/chromosome complex is formed, metaphase commences.



Figure 3: Schematic diagram of the cell cycle ${ }^{1}$

At metaphase, the sister chromatids are assembled to an equatorial position on the mitotic spindle by the dynamic action of the microtubules and associated motor enzymes. This eventual congression at the cellular equator is the net result of both Pmotion (towards the pole) and AP-motion (away from the pole) and can be thought of as a gentle jostling motion to align the chromatids along the equator. The $\mathrm{P}$ movement is driven by a (-)-end directed motor protein in the kinetochore, while the AP movement is driven by a (+)-end motor protein. The kinetochores also contain motor enzymes that destabilise the microtubules, leading to a loss of tubulin heterodimers from the (+) end,

\footnotetext{
II The centromere is the region on the chromomsome where the individual sister chromatids meet
} 
or net microtubule shrinkage. ${ }^{32,34}$ Movement of the microtubules is also dependent upon the inherent microtubule dynamics. ${ }^{30,32,33,34 \mathrm{a}}$

The next stage of cell division is anaphase during which the microtubule dynamics change and the chromatids partition and move to the new spindle poles on the dynamic microtubules, where new cells are being formed. Anaphase microtubule dynamics are particularly rapid, with motor proteins at the poles effectively reeling in the chromosomes by shortening the microtubules. ${ }^{34}$ In this process, duplicate chromosomes are provided to each of the two daughter cells.

\section{3: Tubulin binding agents and cell death}

\subsection{1: The two divisions of antimitotic compounds}

Interruption of microtubule dynamics has proven to be an effective target for cancer chemotherapy. These rapid dynamics are extremely sensitive to tubulininteractive agents that exert their antimitotic action at the metaphase to anaphase transition, possibly by mimicking the actions of naturally occurring regulatory proteins, ${ }^{35}$ figure 4 . Ultimately, this results in arrest at the G2/M transition. Many antimitotic compounds, including the well established chemotherapeutic agents



Figure 4: Blocking of mitotic spindle division by antimitotic compounds ${ }^{1}$ colchicine, the Vinca alkaloids, (including vincristine and vinblastine) and podophylotoxin, operate by interfering with the formation and growth of microtubules during mitosis. ${ }^{36}$ At appropriate concentrations, these drugs inhibit the formation of spindle microtubules or 
depolymerise existing ones. Tubulin is instead diverted into other types of aggregates. This class of antimitotic compounds further includes combretastatin, ${ }^{37}$ maytansine, ${ }^{38}$ rhizoxin, ${ }^{38}$ phomopsin, ${ }^{38}$ the dolastatins, ${ }^{38,39,40}$ cryptonphycins, ${ }^{41}$ benzimidazoles (such as nocadazol), ${ }^{29,31 a, 42}$ the curacins ${ }^{43}$ and the baceterial metabolite RPR $112378 .{ }^{20}$ Selected natural products promoting the depolymerisation of tubulin are shown in figure 5 .



vinblastine<smiles>C=N[C@@H]1CCc2cc(OC)c(OC)c(OC)c2-c2ccc(OC)c(=O)cc21</smiles>

colchicine



rhizoxin<smiles>COc1cc([C@H]2c3cc4c(cc3[C@@H](O)[C@@H]3COC(=O)[C@H]23)OCO4)cc(OC)c1OC</smiles>

podophyllotoxin



RPR1 12378

Figure 5: Selected natural products promoting tubulin depolymerisation

Paclitaxel however, has a mechanism of action unlike any of the tubulin-targeting agents that preceded it to the clinic. In contrast to the antimitotic drugs described above, paclitaxel promotes tubulin-polymerisation, stabilises microtubules and thereby alters normal microtubule dynamics. This results in the formation of abnormal mitotic spindles, mitotic arrest, and the initiation of apoptosis (programmed cell death). ${ }^{44,45}$ At concentrations that are stoichiometric or near-stoichiometric with respect to the 
concentration of tubulin present in solution, paclitaxel causes thick microtubule bundles to form in cultured cells, even in the absence of GTP or MAPs. Once formed, the microtubules are resistant to cold or calcium induced depolymerisation. ${ }^{5}$ Data supports the hypothesis that binding of a molecule of paclitaxel to a tubulin subunit in microtubules induces a conformational change in that subunit that strongly reduces its ability to dissociate when the subunit becomes exposed at the microtubule end. ${ }^{46}$

For more than 15 years paclitaxel, and the structurally related taxanes, were the only compounds known to have this mechanism of action. However, over time, additional drug classes have been shown to act by a similar mechanism. In particular the recently discovered epothilones, identified after screening tens of thousands of compounds for their ability to polymerise tubulin, have been shown to induce tubulin polymerisation in a manner similar to that of pacliltaxel. ${ }^{14,47}$. In fact, when compared to paclitaxel, both epothilones A and B have reported higher potencies as tubulin polymerisation agents, although in vivo studies identified epothilone B as being too toxic for use as an anticancer drug. The lactam analogue of epothilone B however, referred to as azaepothilone $\mathrm{B}$, has shown considerable potential in oncology, and has recently entered phase II clinical trials. ${ }^{48}$

A computer-assisted search for novel compounds with structural analogy to colchicine site inhibitors, also identified the marine natural product (+)-discodermolide, isolated from the sponge Discodermia dissolute, as an antimitotic agent. ${ }^{21,49}$ Indeed, discodermolide-treated breast carcinoma cells displayed spectacular rearrangement of the microtubule cytoskeleton, including extensive microtubule bundling at concentrations one tenth of those required by paclitaxel. ${ }^{21}$ The enhanced bundling observed with both discodermolide and the epothilones when compared with paclitaxel is readily understood in terms of the apparent increased affinity of these compounds for tubulin relative to paclitaxel. 
However, paclitaxel, the epothilones and discodermolide are not the only antimitotic agents that stimulate tubulin polymerisation. Gradually other natural products have been added to this class of compounds. These include laulimalide, ${ }^{18}$ eleutherobin, ${ }^{50}$ sarcodictyin and more, recently peloruside A, figure 6 .





sarcodictyin A


epothilone $B(R=M e)$





eleutherobin



laulimalide



peloruside $\mathrm{A}$

Figure 6: Selected natural products with tubulin polymerisation and microtubule stabilisation properties.

\subsection{2: Mode of action of the antimitotic agents}

It had long been thought that the mechanisms of action of the two classes of compounds were fundamentally different due to the easily recognisable visible effects of either microtuble polymerisation or depolymerisation. However, this concept was 
recently revisited because at low concentrations both classes of compounds appear to inhibit cell proliferation by kinetically stabilising the dynamics of spindle microtubules and not by changing the mass of polymerised material. ${ }^{51}$ This is best illustrated by paclitaxel, which is perhaps the most widely studied antimitotic agent. Numerous studies have shown that in addition to stimulating microtubule polymerisation, paclitaxel also inhibits tubulin exchange at microtubule ends and treadmilling at polymer mass steady state. ${ }^{52,53}$ Such observations however, depend greatly upon the stoichiometry of binding of paclitaxel to the microtubule. ${ }^{46}$ Comparisons between paclitaxel and vinblastine yielded similar results and lead to the hypothesis that differences in the actions of antimitotic drugs may result from the relationship between the stoichiometry of binding of the drug to the microtubules and the degree to which the specific dynamic parameters, such as the extent of growing or shortening, are suppressed.

In the case of paclitaxel, induction of apoptosis has generally been directly associated with $\mathrm{G} 2 / \mathrm{M}$ arrest and occurs as a direct consequence of centrosomal impairment, induction of abnormal spindles and suppression of microtubule dynamics. ${ }^{54}$ However, in some instances, the cell may pass through the $M$ phase of the cell cycle yet arrest at the G1 stage. ${ }^{55}$ Significant debate exists concerning the mechanism by which paclitaxel induces a stable late-G1 block. One scenario involves a critical element of G1 control being sequestered on microtubules, and that the activation of the element requires its release. The release and activation of this element can occur either in a dynamic microtubule system or can be triggered in the absence of microtubules, but would not occur when microtubules are stabilised by paclitaxel. Alternatively, by inducing hyperassembly of microtubules, paclitaxel might impose a checkpoint control upon the cells. Checkpoint controls, which normally function to assure the integrity of the genome, arrest the cell cycle at a discrete point until all prerequisite events have been completed. ${ }^{56}$ However, the triggering of apoptosis by aberrant mitosis or by subsequent multinucleated G1-like state related to mitotic slippage, largely depends on cell type and drug schedule. ${ }^{54}$ Other antimitotic agents, including the epothilones ${ }^{1}$ have also been reported to arrest cells at the $\mathrm{Gl}$ phase of the cell cycle. 
Although numerous studies and their insights have identified a myriad of cellular and molecular effects of antimitotic agents that may offer promise in cancer therapy, these results have often generated contradictory observations. ${ }^{57}$ What is known though is that the cytotoxicity of these antimitotic compounds involves signalling distinct from DNA damaging drugs but common to all microtubule-active drugs with the specific hallmark of $\mathrm{Bcl}-2$ hyperphosphorylation. ${ }^{58.59} \mathrm{Bcl}-2$ is a receptor found on the mitochondrial membrane that plays a regulatory role in apoptosis. The serine/threonine kinase(s) responsible for $\mathrm{Bcl}-2$ phosphorylation, however, have not been conclusively identified.

The controversial role of $\mathrm{p} 53^{\mathrm{III}}$ in paclitaxel-induced apoptosis is still under debate. Although it was initially thought that paclitaxel-induced apoptosis is p53independent $^{60,61}$ again, this depends on cell type and drug concentration. At concentrations too low to inhibit mitotic progression $(3-6 \mathrm{nM})$ paclitaxel can completely inhibit cell proliferation in cells producing higher than normal amounts of p53. Hence, paclitaxel has been shown to compliment the effects of p53 sufficiently to cause arrest at G1 and G2. ${ }^{62}$

\section{4: Biochemistry of peloruside A}

\subsection{1: Cell cycle arrest and effects on the cytoskeleton}

Like its famous predecessor paclitaxel, peloruside A also causes cells to accumulate at the G2/M phase of the cell cycle, as evidenced by an increase in the number of cells in metaphase during flow cytometry studies. ${ }^{17}$ Such observations support the hypothesis that pel A is interacting with the cytoskeleton of cells, in particular, with the microtubules involved in the formation of the spindle. In a manner analogous to the other microtubule stabilisers, pel A causes the formation of microtubules in the absence of microtubule-associated proteins (MAPs) and GTP. Once

III $\mathrm{p} 53$ is a tumour suppressor gene that often plays a crucial role in apoptosis 
formed, the microtubules are resistant to cold or calcium induced depolymerisation. ${ }^{17}$ Figure 7 is a Transmission Electron Micrograph of pel A induced polymerised microtubules, (in vivo preparation). ${ }^{17}$

Although studies comparing pel A with paclitaxel indicated that the arrest in G2/M was more complete for paclitaxel, Western blot results indicate that pel A appears to be more potent than paclitaxel in inducing microtubule polymerisation. ${ }^{17}$ A simple cellular assay was used in which the shift in tubulin from depolymerised (soluble) to polymerised (particulate) forms was followed by electrophoresis and Western blotting of centrifuged soluble



Figure 7: TEM of PelA induced polymerised tubulin (S) and polymerised (P) fractions, figure 8. At $100 \mathrm{nM}$ concentrations, complete tubulin polymerisation was observed for both pel A and paclitaxel. Colchicine, on the other hand, drove the process toward the depolymerised form as expected, figure $8 \mathrm{~A}$. The shift in tubulin dynamics was rapid for both pel $\mathrm{A}$ and paclitaxel, being complete for $100 \mathrm{nM}$ pel $\mathrm{A}$ at or before ' 0 min' (about 30 min after addition of compund to intact cells) and complete for $100 \mathrm{nM}$ paclitaxel at ' 5 min' (35 min after compound addition), figure $8 \mathrm{~B}$.

A



B


Paclitaxel



Peloruside



Figure 8: Western blotting showing the effects of paclitaxel, peloruside $\mathrm{A}$ and colchicine on tubulin polymerisation 
Upon the treatment of human lung carcinoma (H441) cells with pel A, abnormal cytoskeletal rod-like fibres were also observed. Figure 9 shows cells treated for 48 hours with $100 \mathrm{nM}$ peloruside (B) or $100 \mathrm{nM}$ paclitaxel (C). Control cells (A) were left untreated. The fibres did not stain with anti-tubulin or anti-actin ${ }^{\mathrm{IV}}$ antibodies, and thus appear not to be microtubular bundles or actin fibres. Other abnormal cytoskeletal elements, including multiple asters were also observed. ${ }^{17}$ These observations are consistent with studies of this nature on other known tubulin-binders including discodermolide and the epothilones $\mathrm{A}$ and $\mathrm{B}$.


Figure 9: H441 Human lung carcinoma cells showing cytoskeletal bundles. A: Control. B Treated for 48 hours with $100 \mathrm{nM}$ peloruside A. C: Treated for 48 hours with $100 \mathrm{nM}$ paclitaxel

\subsection{2: Cytotoxicity and the identification of apoptotic pathways}

The cytotoxic activity of pel A has been determined in a number of cell lines including the human myeloid leukaemic cell line HL-60, the nontransformed murine myeloid cell line 32D, the human lung adenocarcinoma cell line H441 and 32D cells transformed with ras and bcr/abl oncogenes. $\mathrm{IC}_{50}$ values for pel A ranged from $4 \mathrm{nM}$ to $15 \mathrm{nM}$ in the different cell lines. ${ }^{17}$ These values are comparable to those of other antimitotic drugs.

\footnotetext{
${ }^{\text {IV }}$ Actin is a filamentateous protein found in muscle tissue and in the cytoskeleton of cells
} 
However, considerable work is still required to determine the exact mode of action of pel A. Although the microtubule is presumably the major site of action of pel A, it is not necessarily this particular interaction that kills the cells. A proteomics approach has been used to decipher some of the downstream changes due to the action of pel A, with tryptic digest finger printing identifying a $37 \mathrm{kDa}$ protein altered by both pel $\mathrm{A}$ and pacitaxel, as an isoform of $\alpha$-tubulin. ${ }^{63}$ Further proteomics analysis has revealed points of difference between paclitaxel and pel A, however this work is on-going and awaits further confirmation. Preliminary in vitro studies examining the effects of paclitaxel and pel A on neutrophil superoxide production also revealed differences in the immunosuppressive action of both compounds. ${ }^{64}$ Neutrophils are cells involved with the immune system's response that, among other functions, destroy foreign bodies by producing superoxide radicals. Again, these observations point to differences in the secondary targets of paclitaxel and pel A.

What is perhaps more striking though, are the differing effects of paclitaxel and pel A on the metabolic activity of cells as measured by WST-1 (water soluble tetrazolium) dye reduction. Only metabolically active cells achieve reduction as this requires functional mitochondrial dehydrogenases. Pel A $(180 \mu \mathrm{M})$ stimulated WST-1 dye reduction by $20 \pm 12 \%$, rather than inhibiting it, as might be expected for a potent cytotoxic agent. By contrast, Taxol inhibited WST-1 reduction by $70 \pm 2 \%$ at $120 \mu \mathrm{M}$ but has little effect at $15-30 \mu \mathrm{M} .{ }^{63}$ Such results point to key differences in the initial cellular responses to the two compounds and suggest unique mechanisms of action, depending on concentration and time of exposure.

It is also interesting to note that the sodium borohydride reduction product of pel $\mathrm{A}, 2,{ }^{65}$ does not stabilise microtubules, figure $10,{ }^{17}$ yet is only 30 -fold less cytotoxic than pel A, figure $11 .^{16}$ The observation that 2 causes cell death at nanomolar concentrations without polymerising microtubules




further illustrates the importance of cytotoxic pathways independent of tubulin polymerisation.



Figure 10: Western blot of pel $\mathrm{A}$ and the reduction product 2



Figure 11: Cytotoxicity of pel $\mathrm{A}$ and the reduction product $\mathbf{2}$

\section{5: Tubulin binding sites}

The diverse, seemingly unrelated structures of antimitotic anticancer agents raises the question of whether they bind to the same or different sites on tubulin. Primarily, two very distinct binding sites have been identified, corresponding to the primary mode of action of the antimitotic agent. The first site, found only on unpolymerised tubulin dimer but not the intact microtubule, is the colchicine site. ${ }^{66}$ Many other natural products such as cornigerine, podophyllotoxin, and combretastatin A also bind to the colchicine site, thus preventing tubulin polymeristation. It is also believed that the recently discovered antimitotic agent, RPR 112378 binds to, or near, the colchicine-binding site. ${ }^{20}$ The other predominant binding site is the paclitaxel-binding site..$^{28,67}$ 
Each $\alpha / \beta$-tubulin heterodimer contains one paclitaxel-binding site located between the protofilaments formed from $\alpha$ and $\beta$ tubulin association. Paclitaxel does not bind to free tubulin. ${ }^{68}$ The epothilones, ${ }^{13}$ discodermolide ${ }^{13.69}$ and eleutherobin all inhibit the binding of $\left[{ }^{3} \mathrm{H}\right]$ paclitaxel to tubulin polymers, while laulimalide has been shown to bind to a unique site on B-tubulin. ${ }^{70}$ To date, it is not known if pel A binds directly to microtubules, and if so, whether it binds to the same site as paclitaxel.

\section{6: Multiple Drug Resistance}

Resistance to paclitaxel has generally been associated with either over-expression of the P-glycoprotein efflux pump, ${ }^{71}$ decreased intracellular levels of polymerised tubulin, ${ }^{71}$ or as a result of a mutation of the paclitaxel-binding site on tubulin. ${ }^{72}$ Phosphoglycoprotein is a membrane glycoprotein that functions as a drug efflux pump, thereby reducing the effective intracellular concentration of anticancer drugs. Overexpression of P-gp leads to the multiple drug resistant (MDR) phenotype, wherein tumour cells become simultaneously resistant to a multitude of drugs. ${ }^{13,73}$ Eleutherobin shows similar MDR sensitivity to paclitaxel, however, epothilones, discodermolide, ${ }^{22}$ sarcodictyins and laulimalide look promising in this regard, being only transported to a limited extent by P-gp. ${ }^{13}$ Studies comparing the cytotoxicity of pel A with paclitaxel in cell lines that either overexpress or do not express P-gp are under investigation.

Mutations in the $\beta$-tubulin structural gene results in the resistance of some cell lines to both paclitaxel and the epothilones. ${ }^{696,74}$ Such cell lines include the ovarian carcinoma


respectively. Other selected mutations in similar cell lines affecting paclitaxel cytotoxicity but not discodermolide toxicity have also been reported. ${ }^{72}$ Laulimalide was also active in epothilone- ${ }^{74 \mathrm{a}}$ and paclitaxel-resistant ${ }^{7 \mathrm{~b}}$ cell lines bearing paclitaxel site mutations in the M40 B-tubulin gene. ${ }^{70}$ These studies suggest that even those tubulinbinding drugs that compete with radiolabeled paclitaxel for what appears to be the same binding site, display subtle differences in their interaction with the specific amino acid 
residues of the $\beta$-tubulin binding site. Hence each antimitotic drug offers distinctive advantages over one another with respect to resistant cell lines. It is thus anticipated that pel A will exhibit its own unique cytotoxity with respect to various resistant cancer cell lines.

\section{7: The development of peloruside $A$ as a viable drug candidate}

Although the strategy of using tubulin as a target for cancer chemotherapy has been well established, ${ }^{75}$ different microtubule inhibitors vary in their ability to selectively destroy tumour cells in a clinical setting. While the chemotherapeutic effect of microtubule inhibitors would seem in some way linked to the most evident effect of the drugs, mitotic arrest, there is no direct evidence in support of this assumption - the difference in chemotherapeutic efficacy may lie in more subtle effects. For example both paclitaxel and nocodazole impose mitotic arrest on mammalian cells, ${ }^{55}$ yet paclitaxel has shown promise in chemotherapy, particularly in the treatment of ovarian and breast cancers, while nocadazole has not.

Fortunately though, recent in vivo tests have shown pel A to be efficacious in tumour regression. ${ }^{76}$ A sample of pel A $(5 \mathrm{mg})$ was tested on mice bearing the colon 38 tumour using a single intraperitoneal dose of $40 \mathrm{mg} / \mathrm{kg}$. At this dose, pel A was found to be toxic in four of the five mice, however the remaining mouse showed no toxicity and survived for the duration of the experiment with no evidence of tumour. A further sample of drug ( $3 \mathrm{mg}$ ) was then tested on mice bearing the colon 38 tumour using a lower single intraperitoneal dose of $25 \mathrm{mg} / \mathrm{kg}$. It was again toxic in four of the five mice. The surviving mouse however, showed complete tumour regression with further tumour regression being observed in all the dead mice. Although further studies are required in order to determine the effective dosage in vivo, these results are particularly encouraging because they involved a single bolus dose of pel A, yet anti-cancer treatments normally require multiple doses or intravenous infusion to be effective. 
Whether pel A will equal the utility of Taxol in treating cancer however, can only be answered after significant investment in the drug development and clinical trial process, which ultimately requires an abundant supply of pel A. Due to its scarce natural abundance, it is anticipated that significant quantities of pel A can only be obtained through chemical synthesis. However, at the time of commencing this project, the absolute stereochemistry of pel A was unknown. Ideally, it would be desirable to know the absolute stereochemistry of pel A at the beginning, if not during the early stages of the synthesis, to avoid any possible repetition of work. Thus, methods for determining the absolute stereochemistry of pel A will be discussed before an overview of our synthetic strategies for the synthesis of pel $\mathrm{A}$ is provided. 


\section{8: Determination of the absolute stereochemistry of peloruside A}

Possible methods of determining the absolute stereochemistry of pel A include: a) the synthesis of one enantiomer of pel A followed by comparison of the optical rotation of this enantiomer with that of natural pel $\mathrm{A}$; b) ring opening the macrolide under mild, aqueous conditions, followed by esterification of the resulting carboxylic acid moiety with a chiral reagent and comparison of the absolute stereochemistry at C-2 by ${ }^{1} \mathrm{H}$ NMR spectroscopy in a manner similar to Mosher's method; ${ }^{77.78}$ and c) comparing a synthesised fragment of pel A of known stereochemistry with a similar fragment cleaved from the natural product.

The last strategy initially appeared to be the most viable, with the details of this chosen fragmentation given in scheme 1. This approach involves selectively protecting the primary hydroxyl group of pel A with the tert-butyldimethylsilyl (TBS) moiety, followed by ozonolysis and work-up to produce the side chain fragment $\mathbf{3}$. The synthesis of an identical fragment of known chirality at $\mathrm{C}-18$ has been reported. ${ }^{79}$ Chiral gas chromatography would then be used to compare the retention times of the cleaved side chain 3 and both the synthetically derived racemic, and enantiomerically pure, versions of 3. The attraction of this strategy is that it not only enables the absolute stereochemistry of C-18 to be determined, and hence the absolute stereochemistry of the entire molecule, but also results in the formation of the macrolide segment 4 which may have its own unique biological properties.



1



3) $\mathrm{Me}_{2} \mathrm{~S}$

Scheme 1: Methodology for the determination of the absolute stereochemistry of pel A 


\section{9: Synthetic strategies towards the synthesis of peloruside A}

\subsection{1: Retrosynthetic Analysis}

Although the structure of pel $\mathrm{A}$ is considerably less complex than that of paclitaxel, it nevertheless poses some considerable synthetic challenges, particularly in the formation of the pyranose ring and control of the 10 stereogenic centres. Moreover, pel A provides many opportunities for the discovery and development of new synthetic technologies and strategies. Scheme 2 illustrates the fragmentation of the synthetic target to three main building blocks: the pyranose ring $\mathbf{5}$, commercially available benzyloxyacetic acid $\mathbf{6}$, and the remaining side chain and macrocycle fragment 7 . The two bond disconnections depicted on pyranose ring $\mathbf{5}$ facilitate the synthesis of this core.


Scheme 2: Retrosynthetic analysis of peloruside A 
Formation of pyranose 5a, a synthon for the C-3 to C-11 fragment, would commence with a diastereoselective aldol reaction between the enolate of gem-dimethyl ketone $\mathbf{8}$ and aldehyde 9 to give B-hydroxy ketone $\mathbf{1 0}$, scheme 3. In situ deprotection of the triethylsilyl (TES) moiety under mildly acidic conditions, with subsequent cyclisation, ${ }^{80}$ was envisioned to afford $\mathbf{5 a}$.<smiles>CC(C)(COCc1ccccc1)C(=O)COCc1ccccc1</smiles>



9

Scheme 3: Formation of pyranose 5a

Although the total synthesis of pel $\mathrm{A}$ is outlined in this thesis, the research contains in this thesis involvedd developing a plausible strategy for the synthesis of the C-8 to C-24 carbon backbone of pel A. Accordingly, this required synthesising the two key retrosynthetic fragments, the C-12 to C-24 fragment 7 and the gem-dimethyl ketone fragment $\mathbf{8}$. Progress towards the incorporation of ketone $\mathbf{8}$ into pyranose $\mathbf{5 a}$ is outside the scope of this thesis, however for completeness, strategies for the synthesis and assembly of all the key fragments of pel A will be outlined in the subsequent pages of this chapter.

\subsection{2: Synthesis of the C-3 to C-11 fragment of peloruside A}

Controlling the stereochemical outcome during the formation of the pyranose ring of pel A will be one of the more challenging aspects of the synthesis. First, the desired axial orientation of the C-8 benzyloxy substituent in $\mathbf{5 a}$ requires formation of the $E$ enolate of gem-dimethyl ketone $\mathbf{8}$. Although the close proximity of the gem-dimethyl substituents to the carbonyl group will favour formation of the $Z$ enolate, it is anticipated that the use of lithium tetramethylpiperidide (LTMP), a sterically hindered base, will give the desired $E$ geometry, ${ }^{81}$ scheme 4. 
Controlling the facial selectivity of the subsequent aldol reaction necessitates a transition state involving chelation control of the B-hydroxy substituent. Lithium ${ }^{\vee}$ has been reported to display useful levels of chelation control, ${ }^{82}$ as depicted in scheme 4 . Here lithium chelates to the enolate anion and to both the aldehyde carbonyl oxygen and the B-alkoxy oxygen of the approaching aldehyde. Chelation of the lithium enolate to the approaching aldehyde moiety occurs via a six-membered twist-boat Zimmerman-Traxler transition state, $^{83}$ with coordination of lithium to the OTES substituent resulting in the chair conformation of the aldehyde partner. Chelation occurs with both the gem-dimethyl side chain and the alkyl chain of the B-hydroxy aldehyde adopting an equatorial orientation. Thus, the anticipated net outcome of this reaction is the desired anti-relationship between the C-5 and C-7 and C-7 and C-8 substituents.



10

Scheme 4: Proposed control of the stereochemistry of the pyranose ring by ß-alkoxy lithium chelation

${ }^{v}$ Magnesium has also been shown to have similar levels of chelation control 
If a stereoselective aldol reaction proved unviable, an alternative, although slightly more involved approach for the formation of the pyranose ring has been proposed. An unselective aldol reaction between the enolate of ketone 8 and aldehyde $\mathbf{9}$ would give, after in situ deprotection of the TES substituent, pyranose $\mathbf{1 1}$ as a mixture of diastereomers with the two alkyl chains adopting the equatorial orientation, scheme 5 . Upon methylation, hydrogenation and oxidation, cyclic intermediate 12 would be formed. The C-7 methoxy could then be epimerised to the equatorial position to give $\mathbf{1 3}$ by treatment of $\mathbf{1 2}$ in a basic medium. ${ }^{84}$ At a later stage in the synthesis, the cyclic ketone could be asymmetrically reduced with $\mathrm{NaBH}_{4}$, or other suitable reducing agent, to give the axial conformation of the C-8 ring hydroxyl.



11



3) Oxidation



12



13

Scheme 5: Alternative strategy for the synthesis of the pyranose ring

Two strategies have been proposed for the synthesis of ketone $\mathbf{8}$. The first of these commences with an aldol reaction between glyoxal and isobutyraldehyde, forming the intermediate acetal 14, scheme 6 . The base catalysed thermal rearrangement of $\mathbf{1 4}$ gives hydroxy furanone $\mathbf{1 5}$, which is subsequently reduced to diol $\mathbf{1 6}$, then benzylated to afford 8. Although it is conceivable that difficulties may be encountered in preventing selfcondensation during the first aldol reaction, a patented procedure for this synthesis has been reported. ${ }^{85}$ 




14

15<smiles>CC(C)(COc1ccccc1)C(=O)COCBr</smiles>

8<smiles>CC(C)(CO)C(=O)CO</smiles>

16

Scheme 6: Synthesis of ketone 38 starting with glyoxal and isobutyraldehyde

Alternatively, reduction of commercially available pantolactone $\mathbf{1 7}$, followed by selective benzylation of the primary hydroxy groups and oxidation of the remaining secondary alcohol yields ketone $\mathbf{8}$, scheme 7. Although this three-step synthesis appears to be ideal, difficulties could be encountered in selectively benzylating the primary hydroxyl groups in the presence of the secondary alcohol. Thus the alternative five-step strategy commencing with the protection of pantolactone to give 17 a may be required, scheme 7 . The subsequent reduction of $\mathbf{1 7} \mathbf{a}$, followed by benzylation, deprotection and oxidation yields ketone 8 . Even though the primary hydroxyls could be selectively protected with alternative protecting groups, such as silylanes, ${ }^{86}$ due to literature precedent for the formation of benzloxy enolates ${ }^{87}$ and its overall fit within the synthetic strategy, synthesis of the ketone derivative in its benzylated form was preferred.<smiles>CC1(C)COC(=O)C1O</smiles><smiles>CC(C)(COCc1ccccc1)C(O)COCc1ccccc1</smiles><smiles>[CH2-]</smiles>

$17 \mathrm{R}=\mathrm{H}$

$17 a \quad R=$ Protecting group

Scheme 7: Synthesis of ketone $\mathbf{8}$ starting with pantolactone<smiles>CC(C)(COCc1ccccc1)C(=O)COc1ccccc1</smiles> 
It was proposed that the synthesis of the final fragment of the pyranose ring, aldehyde $\mathbf{9}$, would commence with the mono silylation of commercially available propane-1,3-diol. Oxidation, followed by asymmetric allyl boration using ally diisopinocampheyl-borane, ${ }^{88}$ yields the intermediate alcohol $\mathbf{1 9}$, which is further silylated and ozonolysed, to give aldehyde $9,^{80}$ scheme 8 .<smiles></smiles>

18<smiles>CC=CCC(O)CCO[Pb]</smiles>

19



9

Scheme 8: Synthesis of aldehyde 9

\subsection{3: Sythesis of the C-12 to C-24 fragment of peloruside A}

Two strategies were considered for the synthesis of the C-12 to C-24 fragment of pel A. The key intermediate in the first of these strategies is the keto-lactone $\mathbf{2 0}$, which when reduced, gives the C-12 to C-24 fragment 7, scheme 9. Lactone $\mathbf{2 0}$ results from the ring closing metathesis (RCM) of ester 21 , itself formed from an esterification and aldol condensation reaction, as depicted. For clarity, it should be noted that a regio- and stereoselective aldol reaction between keto-lactone $\mathbf{2 0}$ and the pyranose fragment of pel A would first be conducted before lactone $\mathbf{2 0}$ is reductively opened.



7



20



21

Scheme 9: Initial retrosynthetic analysis for the formation of the C-12 to C-24 fragment 
Amongst the strategies considered for the synthesis of the C-12 to C-24 fragment, the aldol reaction with keto-lactone $\mathbf{2 0}$ was perhaps the most intriguing. Despite a multitude of precedent for the formation of kinetic verses thermodynamic enolates, as the $\alpha$ lactone proton in $\mathbf{2 0}$ is also susceptible to deprotonation, this may result in double bond isomerization and in difficulties with controlling the regiochemistry of the aldol reaction. However, as it was believed that the synthesis of $\mathbf{2 0}$ would be relatively straightforward, this strategy warranted investigation.

In the eventuality of encountering difficulties in controlling the regiochemistry of the aforementioned aldol reaction, the alternative synthesis of the bis-alkoxysilyl ether $\mathbf{2 2}$ was considered for the formation of the C-12 to C-24 fragment, scheme 10. Bis-alkoxy ether $\mathbf{2 2}$ is essentially the "tethered" analogue of $\mathbf{7}$. Initial concerns about the synthesis of this eight membered bis-alkoxysilyl ether by the RCM of the corresponding acyclic precursor 23 were subsequently alleviated by recent publications reporting the synthesis of similar molecules by RCM ${ }^{89,90}$ Diene $\mathbf{2 3}$ could in turn be synthesised by the silylation of B-hydroxyketone $\mathbf{2 4}$ and (S)-3-hydroxymethylpent-1-ene $\mathbf{2 5}$.



22



23



24



25

Scheme 10: Alternative retrosynthesis of the C-12 to C-24 fragment

\subsection{4: The assembly of peloruside $A$}

To accommodate for the variability in the synthesis of the key fragments of pel A, different methodologies for the assembly of these fragments have been developed. In particular, two key strategies have been proposed reflecting the inclusion of the different C-12 to C-24 fragments into the total synthesis. The first of these strategies involves the incorporation of lactone $\mathbf{2 0}$ into the overall synthetic plan, as outlined in scheme 11. 




$5 b$
1) $\mathrm{Ph}_{3} \mathrm{C}^{+} \mathrm{BF}_{4}^{-}$

2) Swern oxidation

3)<smiles>C=C(C[C@H]1OC(=O)[C@H](CC)C=C1C)O[Na]</smiles>

4) $\mathrm{Sml}_{2}, \mathrm{EtCHO}$

5) Methylation



28

1) Hydrolysis

2) Deprotect $R^{1}$

3) Macrolactonisation

4) Deprotection

1)<smiles>[X]OC(=O)COCc1ccccc1</smiles>

2) Methylation



1) $\mathrm{ZnBH}_{4}$

2) a) $\mathrm{TBSCl}$

b) $R^{1} X$

c) $\mathrm{RX}$

3) $\mathrm{KOH}$

4) Swem oxidation $\checkmark$

\section{pelor uside A}

Scheme 11: Synthesis of peloruside A: Strategy 1

To facilitate the coupling of fragment 20a (the TMS enol ether of $\mathbf{2 0}$ ), pyranose 5 a would be first methylated to give $\mathbf{5 b}$, selectively debenzylated, using triphenylmethyltetrafluoroborate, ${ }^{91}$ the ensuing hydroxyl oxidised to an aldehyde under Swern conditions, ${ }^{92}$ and an asymmetric aldol reaction with 20 a conducted. It was envisioned that a Lewis acid catalysed Mukaiyama aldol reaction would be used to control the stereochemistry of this reaction. ${ }^{93}$ A Evans-Tischenko reduction ${ }^{97}$ when then be employed to give the desired C11 to $\mathrm{C}-13$ anti relatioship and the resulting hydroxyl methylated to give $\mathbf{2 6}$, (where $\mathrm{R}=$ $\mathrm{EtCO}_{2}$ ). The lactone would then be reductively opened using $\mathrm{ZnBH}_{4}$, which would also result in the deprotection of the propanyl ester. Thus, this overall transformation would result in three hydroxyl moieties, a primary, allylic and secondary which can be 
selectively protected, before the primary tert-butyldiphenylsilyl protecting group is deprotected $^{94}$ and the resulting hydroxyl oxidised to give aldehyde 27 . An asymmetric aldol reaction between 27 and the enolate of the benzyloxy acid derivative $6 \mathbf{a}$, followed by methylation of the resulting alcohol will follow to yield 21 . It is proposed that a chiral auxiliary (X), such as Evans' oxazolidinone ${ }^{95}$ will exert the necessary $\pi$-facial selectivity required for this aldol reaction. Finally, hydrolysis of the auxiliary, followed by deprotection of the allylic hydroxy substituent, Yamaguchi macrolactonisation, ${ }^{96}$ and deprotection of the remaining protecting groups, including the methoxy acetal, gives peloruside A.

Alternatively, if the C-12 to C-22 bis-alkoxysilyl ether fragment $\mathbf{2 2}$ is incorporated into the synthesis, the order of assembly of the key fragments varies slightly, as depicted in scheme 12 overleaf. First, the tert-butyldiphenylsilyl group on pyranose $\mathbf{5} \mathbf{b}$ will be removed and the resulting hydroxyl oxidised to the aldehyde. An aldol reaction with the benzyloxy derivative $\mathbf{6 a}$, followed by methylation of the newly formed hydroxyl, then gives intermediate 29. Selective clevage of the primary benzyl ether, followed by Swern oxidation, and an asymmetric aldol reaction with bis-alkoxysilyl ether $\mathbf{2 2}$ would then give the complete carbon skeleton 30. An Evans-Tishchenko reduction of $\mathbf{3 0}$ with samarium iodide and propanal, then establishes the correct stereochemistry for the 1,3,5-triol sequence and protects the C-11 hydroxyl as the propanoyl ester. ${ }^{97}$ Methylation of the resulting hydroxyl, followed by desilylation of the bis-silyl ether gives intermediate $\mathbf{3 1}$. Following the hydrolysis of the chiral auxiliary, and propanoyl ester (R), a Yamaguchi macrolactonisation will then be attempted directly with $\mathbf{3 1}$ to give peloruside A. If a mixture containing the 16,21 or the 11 membered lactones is formed, a series of protection and deprotection strategies will be required to selectively protect the C-11 and C-24 hydroxyl groups before the macrolactonisation. 
<smiles>CO[C@H]1C[C@@H](C)OC(O)([C@](C)(Br)COCc2ccccc2)[C@H]1Br</smiles>

$5 b$



4) Methylation



29
1) $\mathrm{Ph}_{3} \mathrm{C}^{+} \mathrm{BF}_{4}$

2) Swem

3)<smiles>CC[C@H]1/C=C(/C)C(CC(C)=O)O[Si](C)(P)OC1</smiles>

22

30



$31 \mathrm{R}=\mathrm{EtCO}$

1) $\mathrm{Sml}_{2}, \mathrm{EtCHO}$

2) Methylation

3) TBAF

\section{1. $\mathrm{R}=\mathrm{E} C \mathrm{CO}$}

1) Hydrolysis

2) Macrolactonisation

pelor uside A

Scheme 12: Synthesis of peloruside A: Strategy 2

Although not without its own unique challenges, the above synthesis appears flexible and convergent enough to enable modifications to be made as required, and at a later date, to facilitate the synthesis of analogues of peloruside A. More recently, several papers concerning the synthesis or partial synthesis or peloruside A have appeared in the literature. These publications are reviewd in the appendix (page 178). 


\section{Chapter Two}

\section{Determination of the absolute stereochemistry of peloruside A}

\section{1: Review}

As previously discussed, determination of the absolute stereochemistry of pel A was to be achieved by determining the stereochemistry at C-18 of the side chain, scheme 13. One possible approach to this requires silylation of the primary hydroxyl of pel A, followed by ozonolysis to produce 2-((tert-butyldimethyl)-siloxymethyl)butanal 3. After synthesising both the racemic and enantiomerically pure versions of $\mathbf{3}$, chiral gas chromatography would first be used to compare the retention times of these derivatives, thus enabling the order of elution of the $(R)$ - and $(S)$ - enantiomers to be determined. By co-injecting a synthetic racemic mixture of $\mathbf{3}$ with the cleaved fragment of pel $\mathrm{A}$, the relative retention time of the cleaved fragment, and hence the chirality at $\mathrm{C}-18$, could then be deduced. Due to the limited abundance of pel A, investigations into this strategy began with the synthesis of the racemic alkyl side chain. This would provide valuable insight into any potential problems that may arise with handling a substrate such as $\mathbf{3}$, and enable the optimum conditions for the separation of the two enantiomers by chiral gas chromatography to be determined.


Scheme 13: Strategy for the determination of the absolute stereochemistry of peloruside A 


\section{2: Strategy One}

The availability of propan-1,3-diol $\mathbf{1 8}$ provided a convenient starting point for the synthesis of the racemic alkyl fragment. Monosilylation of diol 18, ${ }^{98}$ followed by a DessMartin oxidation ${ }^{99}$ resulted in the formation of 3-(tert-butyldimethyl-siloxy)propanal 32, scheme 14. However, due to its apparent volatility, propensity to decompose, and difficulties in finding an adequate stain for thin layer chromatography (t.l.c) analysis, purification of aldehyde $\mathbf{3 3}$ proved to be somewhat troublesome. Nevertheless, eventually gram quantities of $\mathbf{3 3}$ were produced in an overall modest yield.



Scheme 14: Formation of tert-butyldimethylsiloxypropanal

To complete the racemic synthesis of the side chain, an $\alpha$-alkylation reaction of $\mathbf{3 3}$ with ethyl iodide was required. Although the alkylation of aldehydes is problematic, with dialkylation and self-condensation frequently being observed, during the attempted alkylation of $\mathbf{3 3}$ it was nonetheless disappointing to observe none of the desired alkylated adduct $\mathbf{3 b}$. A variety of conditions were investigated, as detailed in scheme 15, however in all instances, the starting material simply decomposed.



33<smiles>CCC(C=O)C[18OH]</smiles>

Scheme 15: Attempted $\alpha$-alkylation reactions 
In an endeavour to synthesise a substrate more amenable to $\alpha$-alkylation, attempts were made to convert 33 into the potentially more robust $N$-cyclohexyl-3-(tertbutyldimethylsiloxy)imine 34 , scheme 16. Although ${ }^{1} \mathrm{H}$ NMR spectroscopic analysis indicated that imine $\mathbf{3 4}$ had indeed been synthesised, on the sub-gram scale, attempts at separating the excess cyclohexylamine from the desired immine proved futile. Consequently, this procedure was abandoned.<smiles>O=CCC[OH+][SbH2]</smiles>

33



$\mathrm{C}_{6} \mathrm{H}_{6}, 4 \AA \mathrm{MS}$<smiles>[SnH3]OCC/C=N\C1CCCCC1</smiles>

34

Scheme 16: Attempted synthesis of $N$-cyclohexyl-3-(tert-butyldimethylsiloxy)imine

In view of these findings an alternative, and indeed more elegant, strategy for the synthesis of $\mathbf{3} \mathbf{b}$ was devised, scheme 17. This involved the reduction of diethylethylmalonate, ${ }^{100}$ monosilylation of the ensuing diol and a Dess-Martin oxidation to form the racemic fragment $\mathbf{3 b}$. Fortunately, these transformations proceeded relatively smoothly. Although literature precedent called for the reduction of the malonate in diethylether at room temperatrure, to ensure complete conversion of the starting substrate to the requisite diol, the reaction mixture was heated at reflux. Yields were comparable to those previously reported. ${ }^{100}$ Unfortunately, yields for the oxidation were once again modest as difficulties were encountered in encouraging this reaction to go to completion whilst preventing the decomposition of starting material. Similar difficulties were encountered with the attempted oxidation of $\mathbf{3 6}$ using pyridium chlorochromate (PCC) as the oxidant. 
<smiles>CCOC(=O)C(CC)C(=O)OCC</smiles>

<smiles>CCC(CO)CO</smiles>

35<smiles>[B-][O+]CC(CC)CO</smiles>

36



$\mathrm{CH}_{2} \mathrm{Cl}_{2}, 44 \%$<smiles>CCC(C=O)CO[Ga]</smiles>

3b

Scheme 17: Alternative strategy for the racemic synthesis of fragment $\mathbf{3 b}$

Having synthesised racemic $\mathbf{3 b}$, investigations into determining the optimum conditions required for the separation of the enantiomers using a $30 \mathrm{~m} \times 0.25 \mathrm{~mm}$ Cyclodex-B ${ }^{\mathrm{TM}}$ chiral gas chromatography column were conducted. As the retention times for the enantiomers were very similar, some problems were encountered when attempting to separate the enantiomers. The apparent instability of aldehyde $\mathbf{3 b}$, which rapidly oxidises to the corresponding acid within a few days (even with storage under argon at $<4^{\circ} \mathrm{C}$ ), also provided an additional hurdle. Numerous changes to the run conditions were required before distinguishable, yet overlapping peaks, for the enantiomers were observed.

Thus with a suitable synthetic strategy for the racemic synthesis of the alkyl side chain in place, work began on the synthesis of the enantiomerically pure alkyl side-chain. Literature precedent ${ }^{79}$ for the synthesis of (-)-(2S)-2-((tert-butyldimethylsiloxy)methyl)butan-1-ol initially involved an enantioselective alkylation reaction using Evan's chiral oxazolidinone 37, titanium(IV) chloride, triethylamine, and trioxane, scheme 18. Subsequent in situ protection of the hydroxyl group using tertbutyldimethylsilylchloride gives $\mathbf{3 8}$. The enantiomeric excess of the alkylation reaction was reported to be greater than $99 \%$, yet the reported overall yield for the two transformations was a mere $48 \%$. 
<smiles>CCCC(=O)N1C(=O)OC[C@H]1Cc1ccccc1</smiles>

37



$\mathrm{Et}_{3} \mathrm{~N}, \mathrm{DMAP}$



$\mathrm{Ph}$<smiles>CCC(C[O+])CO</smiles>

39

Scheme 18: Literature precedent for the synthesis of (-)-(2S)-2-((tert-butyldimethylsiloxy)methyl) butan-1-ol 39

Reproduction of the literature methodology for the titanium tetrachloride mediated $\alpha$ alkylation reaction gave astonishingly poor yields. Grams of substrate $\mathbf{3 7}$ would yield only milligram quantities of the requisite product. In total, only $10 \mathrm{mg}$ of $\mathbf{3 8}$ was ever isolated.

Thus with only a $10 \mathrm{mg}$ sample of $\mathbf{3 8}$, the auxiliary was apprehensively cleaved using a solution of $\mathrm{LiBH}_{4}$ in wet THF. Fortunately this proceeded smoothly. Due to the small sample of the desired alcohol 39, purification of the crude material by flash chromatography was particularly challenging. Nevertheless, $5 \mathrm{mg}$ of pure (-)-(2S)-2((tert-butyldimethylsiloxy)methyl)butan-1-ol 39 was isolated.

Once again, the Dess-Martin oxidation protocol was followed for the oxidation of $\mathbf{3 9}$. This protocol is particularly facile for the oxidation of milligram quantities of substrate. After a simple filtration of the crude reaction mixture through a Pasteur pipette sized silica gel plug, a sufficient quantity of the enantiomerically pure aldehyde $\mathbf{3} \mathbf{c}$ was obtained for analysis by gas chromatography; scheme 19. Thus, with great anticipation, enantiomerically pure $\mathbf{3 c}$ and a freshly prepared sample of the racemic aldehyde $\mathbf{3 b}$ were co-injected into the gas chromatogram.<smiles></smiles>

39



$\mathrm{CH}_{2} \mathrm{Cl}_{2}$<smiles>CCC(C=O)CO[SbH3]</smiles>

$3 c$

Scheme 19: Final oxidation of the enantiomerically pure alcohol 
Although separation of the enantiomers by gas chromatography was not ideal, it was nevertheless possible to conclude that the $(S)$-enantiomer was the first enantiomer eluted, as evidenced by an increase in the peak height of the first of the two eluted peaks. Thus all that remained in this strategy was to silylate pel A with the tert-butyldimethylsilyl group then cleave the olefinic bond, via ozonolysis, to form the corresponding aldehyde.

Unfortunately this was where considerable difficulties arose. Due to the $1 \mathrm{mg}$ sample size of pel A that was available, silylation proved unviable, scheme 20. Presumably this was due to the dilution at which the reaction was conducted - effective silylation requires concentrated conditions. ${ }^{101}$ Although we acknowledged that small-scale reactions are often troublesome and present their own unique practical difficulties, this was nevertheless an exceptionally disappointing result. An alternative approach was required.



1

40

Scheme 20: The attempted silylation of peloruside A 


\section{3: Strategy Two}

Two factors were of utmost concern when redefining the target molecule. First, a protecting group had to be chosen that had a much greater likelihood of being incorporated onto a natural product on such a small scale, and second, there had to be a facile synthesis for the corresponding enantiomerically pure side chain. Although one could contemplate simply performing ozonlysis on unprotected pel $\mathrm{A}$, there were some concerns with this strategy. While an Oppenauer-type oxidation of diol 35 using $\mathrm{Cp}_{2} \mathrm{ZrH}_{2}$ could be conducted, scheme $21,{ }^{102}$ formation of the required enantiomer would be difficult and would ultimately require the manipulation of (-)-(2S)-2-((tert-butyldimethylsiloxy)methyl)butan-1-ol 39, or similarly protected alcohol. Given the difficulties in synthesising the siloxy alcohol, further chemical manipulations on this segment were unpractical. It was also conceivable that $\mathbf{4 1}$ would be a difficult molecule to work with due to its low molecular mass and highly polar nature. Consequently, this strategy was discarded.<smiles>CCC(CO)CO</smiles>

35



Cyclohexanone<smiles>CCC(C=O)CO</smiles>

41

Scheme 21: Possible Oppenauer-type oxidation of $\mathbf{3 5}$

Literature precedent for the acetylation of many natural products ${ }^{103}$ prompted us to believe that the primary hydroxyl group of the side chain of pel A would also be amenable to acetylation. However unlike the previous silylation strategy, in which only the primary hydroxyl terminus was expected to be protected, acetylation of pel A would ultimately result in the acetylation of many, if not all, of the free hydroxyls in pel A. Although it was initially desirable to retain the remaining portion of pel $\mathrm{A}$ in its unprotected form after oxidative cleavage (for potential biological testing), this was not of fundamental importance to the strategy. Thus, a review of the literature was conducted to determine if there was synthetic methodology for the synthesis of the enantiomerically pure acetylated 
alkyl side chain. Fortuitously, such methodology had been reported, ${ }^{100}$ as outlined in scheme 22. Here the lipase, extracted from the microorganism Pseudomonas cepacia, kinetically deacetylates, and hence resolves, the diacetylated precursor, $\mathbf{4 2}$. Alcohol $\mathbf{4 3}$ is subsequently oxidised under Swern conditions to give enantiomerically pure $\mathbf{4 4}$.



42<smiles>CC[C@H](C=O)COC(C)=O</smiles>

44

Scheme 22: Alternative strategy for the synthesis of an enantiomerically pure side chain 44

Thus with a plausible strategy for the synthesis of the enantiomerically pure acetylated side chain in place, all that remained was a methodology for the synthesis of the racemic acetylated side chain. Needing both the monoacetylated alcohol $\mathbf{4 3 b}$, (for the racemic synthesis) and the diacetylated alcohol $\mathbf{4 2}$ (for the enzymatic resolution), a solution of diol 35 was acetylated under standard conditions but with only 1.2 equivalents of acetyl chloride, scheme 23. This produced an easily separable mixture of the monoacetylated and diacetylated alcohols, $\mathbf{4 3 b}$ and $\mathbf{4 2}$ respectively, in a 1:1 ratio. Although there was literature precedent for the oxidation of $\mathbf{4 3 b}$ under Swern conditions, the Dess-Martin oxidation protocol had been effectively adapted to meet our requirements for small-scale sample sizes and therefore this later oxidation protocol was followed. However like its silylated counterpart, $\mathbf{4 4 b}$ was also prone to decomposition and was difficult to detect via t.l.c. Numerous stains were screened ${ }^{104}$ before a combination of reagents, saturated $\sigma$ dianisidine in glacial acetic acid, was found to be suitable. 
$\widehat{\mathrm{HO}} \widehat{\mathrm{OH}}$

35



$80 \%$ total yield<smiles>CCC(CO)COC(C)=O</smiles>

$43 b$<smiles>CCC(COC(C)=O)COC(C)=O</smiles>

42<smiles>CCC(COC(C)=O)C(=O)O</smiles>

44b

Scheme 23: Formation of the acetylated racemic fragment $44 \mathrm{~b}$

Thus with the racemic aldehyde in hand, attempts were then made at separating the two acetylated enantiomers using the $30 \mathrm{~m} \times 0.25 \mathrm{~mm}$ Cyclodex-B ${ }^{\mathrm{TM}}$ column. Although numerous changes were made to the operating conditions during the GC studies, regrettably, these enantiomers were practically inseparable with this particular column. After some consideration, a decision was made to invest in a $60 \mathrm{~m}$ x $0.25 \mathrm{~mm}$ Cyclodex$\mathrm{B}^{\mathrm{TM}}$ column as it was anticipated that the increased length of the column would result in a greater separation of the two peaks for the eluted enantiomers. Thankfully this assumption came to fruition. The acetylated enantiomers could indeed be separated when using the 60 $\mathrm{m} \times 0.25 \mathrm{~mm}$ Cyclodex-B ${ }^{\mathrm{TM}}$ column, albeit after an extended two hour run time! The optimum run conditions were as follows: Inlet: Split $275^{\circ} \mathrm{C}$; Detector: FID $320^{\circ} \mathrm{C}$; Carrier gas: hydrogen; Flow: $35.8 \mathrm{~cm}^{3} \mathrm{~s}^{-1}$; Temperature programme: Isothermal at $60^{\circ} \mathrm{C}$.

Having established the conditions required for the adequate separation of the two acetylated enantiomers, our focus then shifted towards the enzymatic resolution of the diacetylated precursor $\mathbf{4 2}$. As surmised in the literature, ${ }^{100}$ diacetate $\mathbf{4 2}$ readily undergoes hydrolysis into the racemic monoacetate in the absence of lipase $P S$, (thus $\mathbf{4 2}$ must be added to an aqueous solution of lipase PS buffered at $\mathrm{pH} 7.2$ ). Although the literature reported enantiomeric excesses of greater than $88 \%$ at $69 \%$ hydrolysis, the cost of the 
enzyme meant that this reaction could only be conducted at $5 \%$ of the scale reported. Consequently, it was a little more difficult to monitor the hydrolysis rate and the ee value, ${ }^{\mathrm{VI}}$ using only $100 \mathrm{mg}$ of the lipase, was only $72 \%$. This result however, was still adequate.

Thus with only $40 \mathrm{mg}$ of enantiomer 43 available, and with the knowledge that the corresponding aldehyde $\mathbf{4 4}$ has limited stability, a small $(8 \mathrm{mg}$ ) sample of purified $\mathbf{4 3}$ was subjected to a Dess-Martin oxidation. Once again, this methodology proved to be successful with milligram quantities of aldehyde 44 being produced after purification though a small (pasteur pipette sized) silica gel plug, scheme 24.



Scheme 24: Synthesis of the acetylated, enantiomerically enriched side chain.

Concurrently, racemic 2-(acetoxymethyl)butanal was synthesised and the racemic and enantiomerically pure fragments co-injected into the gas chromotograph. As anticipated, the two samples co-eluted. The $(R)$ enantiomer elutes first from the column with a retention time of 116.5 minutes, while the $(S)$-enantiomer has a longer retention time of 119.5 minutes. The gas chromatograph trace of racemic aldehyde $\mathbf{4 4 b}$ coinjected with the $(S)$ enantiomer $\mathbf{4 4}$ is given in figure 12.

\footnotetext{
${ }^{\mathrm{V}} \mathrm{The}$ enantiomeric excess was determined by g.c analysis of the corresponding aldehyde
} 




Figure 12: Gas chromatograph trace of the racemic aldehyde $44 \mathrm{~b}$ co-injected with the $(S)$-enantiomer $\mathbf{4 4}$

Once again, the point in the strategy had now been reached where pel A had to be manipulated. But this time only $0.5 \mathrm{mg}$ of pel A was available. However, even before any attempts at acetylating pel A were made, two important questions needed to be answered. First at this scale, would the final enantiomer even be detectable by the gas chromatograph, and second, how feasible were small-scale ozonolysis reactions?

To determine the sensitivity of the gas chromatograph, some approximate concentration studies were conducted. Due to the instability of $40 \mathrm{~b}$ and the 2 hour run times required for good peak resolution, pinene was instead chosen as a standard. Fortunately, $3 \mu \mathrm{L}$ aliquots taken at dilutions as low as $0.01 \mathrm{mg}$ of pinene per $1 \mathrm{~mL}$ of dichloromethane could be detected by the GC. This equated to $2.2 \times 10^{-10}$ moles of substrate and indicated that, when starting with a $0.5 \mathrm{mg}$ sample of pel A $\left(9.11 \times 10^{-7}\right.$ moles $)$, the cleaved acetylated side chain should be detected, even if the acetylation and ozonolysis reactions went to only $10 \%$ completion. 
The easily prepared model compounds $\mathbf{4 5}$ and $\mathbf{4 6}$, figure $\mathbf{1 3}$, were then synthesised in order to investigate small-scale ozonolysis reactions. What became apparent in these studies was that the length of time that the substrate was exposed to ozone was crucial between two and three seconds was ideal for samples sizes of less than $1 \mathrm{mg}$. Furthermore, triphenylphosphine, as compared to dimethylsulphide, appeared to be a better reagent by which to reductively work-up the reaction. Nevertheless, these were difficult reactions. Undesired side products were always formed, and there was a fine balance between the complete conversion of the alkenes to the corresponding aldehydes with additional side products, or incomplete reactions and fewer side products. Isoprene was added to the reaction mixture in an attempt to "mop-up" the excess ozone, although this proved futile. All the same, as much experimenting as was practical had been conducted and the acetylation of pel A was attempted.



45



46

Figure 13: Model substrates for small-scale ozonolysis reactions

A $0.5 \mathrm{mg}$ sample of pel A was thus dissolved in $1 \mathrm{ml}$ of a $1: 1$ mixture of acetic anhydride:pyridine, then left to stir for 18 hours at room temperature in the dark, scheme 25. The solution was then passed through a small glass column packed with HP-20S (polystyrene divinylbenzene) beads and the eluent combined with $3 \mathrm{~mL}$ of distilled water and passed through the same column. The resulting eluent was diluted with a further $3 \mathrm{~mL}$ of water and passed through the column. The column was then washed with approximately $25 \mathrm{~mL}$ of water to remove most of the remaining pyridine and acetic anhydride, then $10 \mathrm{~mL}$ of $20: 1$ water:acetone, and $2.5 \mathrm{~mL}$ of $1: 1$ water:acetone to remove any unacetylated pel A. The reaction product was then eluted with $6 \mathrm{~mL}$ of acetone and the solvent removed under reduced pressure. Silica gel t.l.c analysis of the crude material revealed the presence of two major spots with $R_{f}$ values of approximately 0.50 to 0.55 in 
$5 \% \mathrm{MeOH}: \mathrm{CH}_{2} \mathrm{Cl}_{2}$. It was believed that these two products were the tetra and penta acetylated adducts. The crude material was then purified further by silica gel chromatography using a solvent gradient from pure $\mathrm{CH}_{2} \mathrm{Cl}_{2}$ to $10 \% \mathrm{MeOH}: \mathrm{CH}_{2} \mathrm{Cl}_{2}$. Due to the exceptionally small sample size, the two major products were collected as one fraction.



Scheme 25: Acetylation of peloruside A

Spectral analysis of the combined products by ${ }^{1} \mathrm{H}$ NMR revealed the presence of two major acetyl methyl peaks at $\delta 2.24$ and $\delta 2.07$, three peaks of slightly lower intensity at $\delta$ $2.19, \delta 2.14$ and $\delta 2.22$, and several other minor acetyl peaks in the same region of the spectrum, Characteristic peaks of pel A, such as the methoxy peaks between $\delta 3.3$ and $\delta$ $3.5 \mathrm{ppm}$ were also present in spectrum. This suggested that although we had not cleanly acetylated every free hydroxyl of pel A, we speculated that the least sterically encumbered primary hydroxyl was definitely acetlyated. Due to the small sample size, we were unable to obtain any other conclusive evidence that we had acetylated pelA, however chromatographic analysis most definitely suggested that pel A had been acetylated. For example, native pel A elutes from HP-20S beads with a 3.3:1 solution of acetone:water. ${ }^{65}$ Our sample however, was washed with a 1:1 solution of acetone:water, which would most certainly remove any unacetylated pel A. The less polar acetylated adduct however, would remain on the column. Furthermore, pel A cannot be eluted from a silica gel column when using a solvent gradient from pure $\mathrm{CH}_{2} \mathrm{Cl}_{2}$ to $10 \% \mathrm{MeOH}$. 
$0.25 \mathrm{mg}$ of the purified acetylated pelA was then subjected to ozonolysis, scheme 26 , with a sample from the crude reaction mixture being subsequently injected into the gas chromatograph. The initial GC trace looked encouraging - an exceptionally small peak with a retention time of approximately $116 \mathrm{~min}$ was observed. Although this differed slightly from the retention times of 116.5 and 119.5 minutes previously reported, we postulated that this was within experimental error and subjected a second sample, spiked with a freshly prepared sample of the racemic aldehyde, to GC analysis. Unfortunately, in this and subsequent runs, three peaks were observed. Unbelievably, an impurity present had a retention time within 20 seconds of the desired enantiomers.



$1 a$



44



$4 a$

Scheme 26: Attempted ozonolysis of acetylated peloruside A

To say the least, these results were tremendously disappointing. In an attempt to determine why the desired compound was not detected, a small sample of the racemic aldehyde was subjected to our ozonolysis conditions. Although in theory it is the intermediate ozonolide, and not the subsequent aldehyde, that will be exposed to ozone, it was important to determine how stable the resulting aldehyde is in the presence of excess ozone. Indeed, the ${ }^{1} \mathrm{H}$ NMR of our crude product told an interesting story - there was no sign of the aldehyde substrate. Obviously, on such a small scale, even the smallest trace of excess ozone would destroy our desired compound. It became apparent that on this scale, and with our particular substrate, this was not a plausible strategy. 
Although this strategy was beginning to look particularly dubious, after investing so much time into this project it seemed careless to not to at least consider one last option - namely that of a dihydroxylation of the olefinic double bond using osmium tetroxide followed by a periodate oxidative cleavage. ${ }^{105}$ As I was unwilling to take any uncalculated risks, model studies on a trisubstitued alkene closing resembling that of the side chain of pel A first needed to be conducted. Indeed, such a substrate could be synthesised by performing a Wittig $^{106}$ reaction on aldehyde $44 \mathrm{~b}$ using triphenylphosphoranylidene isopropane. However, to add to the frustration, this meant returning to the starting point of the synthesis as all of the precursors had been consumed.

Thus aldehyde 44b was synthesised in a manner similar to that previously discussed, although, due to the larger scale, the Swern oxidation procedure was used, scheme 27. Although there was literature precedent for this synthesis, ${ }^{100}$ it was found that a slight modification of the literature procedure was required to give yields similar to those previously reported. It is interesting to note that the yields for both the Dess-Martin and Swern oxidations of alcohol $\mathbf{4 3 b}$ are comparable.



Scheme 27: Swern oxidation of alcohol 39b

Isopropyltriphenylphosphonium bromide, synthesised according to literature procedure, ${ }^{107}$ was then used as the precursor for the in situ formation of triphenyl-phosphoranylidene isopropane, scheme 28 overleaf. Aldehyde $44 \mathrm{~b}$ was subsequently added to the ylide, with the model alkene 47 being synthesised in modest yield. 




Scheme 28: Synthesise of the model alkene substrate 47

Alkene 47 was then subjected to oxidative cleavage using $10 \mathrm{~mol}_{\%} \mathrm{OsO}_{4}$ and sodium periodate, scheme 29 . Fortunately, on the $15 \mathrm{mg}$ scale, the required aldehyde $44 \mathrm{~b}$ was isolated and characterised. This reaction was then scaled down to the $0.5 \mathrm{mg}$ scale and analysed via gas chromatography. Again results looked promising, with two peaks eluting with the same retention times as those for the racemic aldehyde.<smiles>CCC(C=C(C)C)COC(C)=O</smiles>

47



2) $\mathrm{NaIO}_{4}, \mathrm{rt}, 90 \mathrm{~min}$<smiles>CCC(C=O)COC</smiles>

$44 b$

Scheme 29: Model studies for the oxidative cleavage of pel A

After preparing a second sample of acetylated pel A, all that remained was the oxidative cleavage of the double bond of pel A. Thus, a $0.5 \mathrm{mg}$ sample of acetylated pel A was subjected to the dihydroxylation conditions previously devised for the model studies, scheme 30 . The crude sample was subsequently injected into the gas chromatograph. Upon observing a single peak near those of our desired enantiomers, a second sample, spiked with the racemic aldehyde was injected. 




1a



2) $\mathrm{NalO}_{4}, \mathrm{rt}, 90 \mathrm{~min}$

Scheme 30: Attempted osmium/periodate olefinic cleavage of peloruside A

Disappointingly, the peaks did not co-elute. There was no evidence of the desired cleaved side chain fragment from pel A, only an impurity that again had a retention time within 20 seconds of the desired enantiomers. The source of this impurity remained a mystery. It was not observed in the previous model studies.

We could only speculate as to why this strategy failed. Perhaps the reaction conditions weren't specific enough for the cleavage of the olefinic bond in pel A and alternative reactions, that ultimately destroyed the desired fragment, occurred. In any case, with a limited natural abundance of pel A, it was not feasible to repeat these experiments. From the observed results it appeared that a larger sample size of pel A was required if this strategy was to succeed. Consequently, the project was discontinued. ${ }^{\mathrm{VII}}$

\footnotetext{
VII The total synthesis, and hence determination of the absolute stereochemistry, of pel A was subsequently published by Xibin Liao, Yusheng Wu and Jef K. De Brabander; see Angewante Chemie, Int. Ed. Engl. 2003, 42, 1648. The absolute configuration of pel A was reported to be opposite to the one shown throughout this thesis
} 


\section{Chapter Three}

\section{Synthesis of the C-8 to C-11 fragment of Peloruside A}

\section{1: Strategy One}

Amongst the strategies considered for the synthesis of the gem-dimethyl ketone $\mathbf{8}$, the aldol reaction between glyoxal and isobutyraldehyde appeared to offer the most potential as the starting point in our synthesis, scheme 31 . Although this strategy has the advantage in that it does not require the regioselective protection of primary alcohols in the presence of a secondary alcohol, it was quickly established that the formation of $\mathbf{1 4}$ was more difficult than originally envisioned. The large-scale patent procedure ${ }^{85}$ that was followed for the formation of $\mathbf{1 4}$ appeared to be unsuitable on the multigram scale. Rather than cleanly producing $\mathbf{1 4}$, a menagerie of aldol condensation and self-condensation was observed. The methodology required for the successful implementation of this protocol appeared to require very precise conditions. Determining these exact conditions would be a particularly laborious process and hence, an avenue we were not prepared to explore at this stage.


Scheme 31: Synthesis of the gem-dimethylketone 8: Strategy One 


\section{2: Strategy Two}

Consequently, we focussed our attention on the formation of gem-dimethylketone 8 from pantolactone $\mathbf{1 7}$, scheme 32 . Literature precedent indicated that the reductive ring opening of 17 could be achieved using a solution of lithium aluminium hydride $\left(\mathrm{LiAlH}_{4}\right)$ in THF. ${ }^{108}$ However in our hands, the yields for this procedure were disappointingly low even though several attempts were made at this strategy. Consequently attempts were made at reducing the lactone using a solution of $\mathrm{NaBH}_{4}$ in ethanol, but again, the yield of the desired triol was exceptionally low.



17



8

Scheme 32: Proposed route for formation of gem-dimethyl ketone $\mathbf{8}$

Our limited success in reducing unprotected pantolactone $\mathbf{1 7}$ prompted us to investigate the reductive ring opening of a suitably protected species, scheme 33 . We felt that the highly polar nature of the triol and the workup conditions, which requires filtration through a small silica gel plug, may have lowered the yields. Although the protection of pantolactone would add additional steps to the synthesis, the protected diol 48 has the advantage in that regioselective protection strategies are no longer required when protecting the primary alcohols.<smiles>[3H][C@]1(C)COC(=O)C1O[13C](=O)[O-]</smiles>

Scheme 33: Modified strategy for the formation of gem-dimethylketone $\mathbf{8}$ 
The ability to perform a one-pot desilylation-oxidation ${ }^{109}$ of aliphatic tertbutyldimethylsilyl ethers using catalytic quantities of $\mathrm{PdCl}_{2}\left(\mathrm{MeCN}_{2}\right.$ prompted us to first investigate the protection, and subsequent reduction of the silyl protected lactone $\mathbf{1 7} \mathbf{a}$, scheme 34. Although protection of lactone 17 as the silyl ether proceeded smoothly, initial investigations within our laboratory into the reduction of this lactone were disappointing. Upon reduction of the lactone the silyl group preferentially migrated to the primary terminus. ${ }^{110}$ In many respects this was not an entirely unexpected observation as the ability of a silyl group to migrate from one hydroxyl to another is not uncommon and frequently causes problems in organic synthesis. ${ }^{111}$ In fact, the TBS group is particularly renowned for its migratory ability. ${ }^{112}$ Thus, an alternative protecting group was sought.

Accordingly, it was proposed that a tosyl group would suffice as a protecting group. Even though one may question the wisdom of reducing the lactone in the presence of the tosyl functionality, literature precedent existed for the tosylation of pentalactone $\mathbf{1 7}$, followed by reductive ring opening using diisobutylaluminium hydride (DIBAL-H). ${ }^{113}$ Thus this strategy warranted investigation, scheme 34 . Although synthesis of protected lactone $\mathbf{1 7} \mathbf{b}$ proceeded smoothly in yields comparable to those reported in the literature, in our hands, the subsequent reductive ring opening using DIBAL-H was unsuccessful. Diol $\mathbf{4 8 b}$ was only ever formed in abysmal yields, even after the addition of excess DIBAL-H and the implementation of longer reaction times.


$$
\text { 17a: } R=\text { TBS }
$$

17b: $R=T s$

48a: $R=T B S$

48b: $R=T s$

Scheme 34: Protection of pantolactone 17 and attempted reduction

Consequently, alternative reduction protocols were investigated. The portion-wise addition of $\mathrm{NaBH}_{4}$ to a solution of the tosyl protected lactone $\mathbf{1 7} \mathbf{b}$ in $\mathrm{THF}$ at $0{ }^{\circ} \mathrm{C}$ produced a myriad of undesired side products, with no trace of the desired diol $\mathbf{4 8} \mathbf{b} .{ }^{1} \mathrm{H}$ 
NMR of the crude product suggested that the lactone had not undergone ring opening. Similar results were observed when lithium borohydride $\left(\mathrm{LiBH}_{4}\right)$ was added in portions at $0{ }^{\circ} \mathrm{C}$ to a solution of $\mathbf{1 7 b}$ in THF. Although a solution of lactone $\mathbf{1 7} \mathbf{b}$ and $\mathrm{LiAlH}_{4}$ in refluxing THF primarily resulted in the decomposition of the lactone, there was some evidence by ${ }^{1} \mathrm{H}$ NMR that the lactone had undergone reductive ring opening.

Even though these results were less than desirable, they suggested that if a more stable protecting group was used, the lactone might be successively reduced using a solution of $\mathrm{LiAlH}_{4}$ in refluxing THF. Being rather chemically inert, it was therefore postulated that an ether-protecting group would be suitable. In particular, an allyl ether was chosen for this purpose as there was some evidence for dual deprotection/oxidation at a later stage in the synthesis. ${ }^{114}$

A standard Williamsons ether synthesis protocol, ${ }^{115}$ using allyl bromide as the alkyl halide, was followed to synthesise the allyl protected lactone. The use of the more polar DMF, rather than THF, as a solvent produced optimal results. However, due to an untimely quench of the NMR magnet, we were unable to conclusively prove that the desired allyl protected lactone had been synthesised. Conclusive mass spectral data on the compound was also unattainable. Nevertheless, due to the interests of time, and from IR and t.l.c analysis, it was concluded that the hydroxyl functionality had been protected with an allyl group, and we decided to continue on with the synthesis.

Thus, the next step in this strategy was the reduction of the allyl protected lactone. Based on our previous observations, $\mathrm{LiAlH}_{4}$ was initially chosen as a reducing agent. Fortunately, after a little experimentation with the reaction conditions, the lactone was successfully reduced in a modest $57 \%$ yield. The workup procedure in this synthesis was important, requiring the quenching of the reaction with hydrated sodium sulfate followed by filtration though a small silica gel plug for optimum yields.

Having successively reduced the lactone, the next functional group transformation involved the protection of the two primary hydroxyl groups. As there is literature 
precedent for the formation of $\beta$-benzyloxy enolates,${ }^{87}$ and the commercially available benzyloxyacetic acid is also a key fragment in our retrosynthetic analysis, it was decided that the protection of the hydroxyl groups as benzyl ethers was most appropriate. This would allow for a global deprotection of the benzyl groups upon the completion of our synthesis. Consequently our proposed diol was benzylated, again following Williamson's ether synthesis protocol, in $77 \%$ yield.

Thus at this stage of the synthesis, strategies for the deprotection of the allyl group were investigated. Ideally, removal of the allyl group can be effected by metal catalysed isomerization of the allyl ether to the corresponding propenyl ether, with subsequent $\mathrm{H}^{+}$ or $\mathrm{Hg}^{2+}$ hydrolysis ${ }^{116}$ or oxidative cleavage. ${ }^{117}$ Appropriate metal catalysts include palladium on carbon, ${ }^{118}$ palladium tetrakis-triphenylphosphine, ${ }^{119}$ and $t r i s$ triphenylphosphine rhodium(I) chloride (Wilkinson's catalyst). ${ }^{120}$ The aforementioned strategies were thus attempted on our allyl-protected substrate, but with little success. Consequently alternative strategies, including the use of sodium borohydride and iodine (it is believed that $\mathrm{NaBH}_{4} / \mathrm{I}_{2}$ in THF is a source of diborane which initially coordinates to the ethereal oxygen), ${ }^{121}$ oxidative cleavage using selenium dioxide and acetic acid ${ }^{122}$ or titanium trichloride and magnesium, ${ }^{123, \mathrm{VIII}}$ were investigated. Allyl transfer reactions, involving palladium chloride and acetic acid ${ }^{124}$ or palladium chloride, copper chloride and $\mathrm{O}_{2}{ }^{125}$ were also attempted. Unfortunately, none of these were successful.

Not surprisingly, these results were particularly puzzling. However just as frustration set in, we obtained access to another NMR instrument and were able to fully analyse the synthetic precursors. This provided us with some valuable information. Both ${ }^{1} \mathrm{H}$ and ${ }^{13} \mathrm{C}$ NMR analysis revealed that even though we had protected the hydroxyl group of the starting lactone 17, the initial allyl protected lactone underwent a subsequent $\alpha$ substitution reaction, forming the di-substituted species $\mathbf{5 0}$, scheme 35 . Thus, our previous reduction and benzylation strategies, as discussed above, had actually been

\footnotetext{
VIII Although $\mathrm{TiCl}_{3}$ and $\mathrm{Mg}$ is also known to cleave benzyl ethers, the cleavage of allyl ethers is more facile as the $\eta 3$-allyl complexes formed in the reaction are more stable than the corresponding $\eta 3$-benzyl complexes; see reference 123
} 
conducted on the disubstituted species $\mathbf{5 0}$. Suddenly the discrepancies in our data and the potential problems with our methodology became glaringly obvious.



17



49



50



52

51

Scheme 35: Formation of the dialkylated species

In an attempt to prevent disubstitution from occurring, for every mole of lactone used the number of moles of $\mathrm{NaH}$ and allyl bromide were limited to 1.1 and 1.05, respectively. Previously four moles of $\mathrm{NaH}$ and three moles of allyl bromide had been used. For the most part, these conditions prevented disubstitution from occurring, however they also resulted in incomplete reactions. These problems were circumvented by the use of 1 equivalent of potassium-tert-butoxide in a $1: 1$ solution of THF/DMF, followed by the addition of 1.1 equivalents of allyl bromide. The desired allyl protected lactone $17 \mathbf{c}$ was cleanly produced in $82 \%$ yield, scheme 36 . Initially we proposed that the steric bulk of the base may favour mono-substitution, although it was later found that an excess of the base would also result in the disubstituted adduct. ${ }^{126}$ Thus, simply the ability to accurately measure one equivalent of potassium-tert-butoxide, when compared to $\mathrm{NaH}$, resulted in the clean formation of the desired mono alkylated adduct $\mathbf{1 7} \mathbf{c}$. 
Fortunately the techniques developed for the reduction of the lactone $\mathbf{5 0}$ were also applicable for the reduction of the allyl protected lactone $17 \mathbf{c}$. In fact, when compared to the reduction of $\mathbf{5 0}$, the yield for the reduction of $\mathbf{1 7 c}$ improved dramatically with the desired diol $\mathbf{5 3}$ being synthesised in a respectable $77 \%$ yield. Subsequent benzylation of $\mathbf{5 3}$ again proceeded smoothly with the dibenzylated species $\mathbf{5 4}$ being synthesised in $\mathbf{9 0 \%}$ yield, scheme 36 .<smiles>CC1(C)COC(=O)C1O</smiles>

17
1) $\mathrm{KO} \mathrm{B}^{\mathrm{B}} \mathrm{Bu}, 1 \mathrm{eq}, \mathrm{DMF} / \mathrm{THF}$ $0^{\circ} \mathrm{C}, 30 \mathrm{~min}$


$81 \%$



$17 \mathrm{c}$



1) $\mathrm{NaH}, 5 e q, \mathrm{DMF}$

$\mathrm{O}^{\circ} \mathrm{C}, 30 \mathrm{~min}$

2) $\mathrm{BnBr}, 2.05 \mathrm{eq}$ rt, $14 \mathrm{~h}$ $90 \%$



54

Scheme 36: Synthesis of the benzyl protected allyl ether $\mathbf{5 4}$

With the allyl ether $\mathbf{5 4}$ successfully synthesised, the next major challenge was removing the allyl group. As alluded to earlier, it was proposed that a two-step, one pot, dual deprotection/oxidation of the allyl ether $\mathbf{5 4}$ was plausible. Although such methodology is sparse, there is literature precedent indicating that the combination of tert-butyl hydroperoxide (TBHP) and $\mathrm{CrO}_{3}$ initially deprotects an allyl group, then oxidises the resulting alcohol. ${ }^{114}$ One can infer from the literature that this deprotection arises from the allylic hydroxylation of the allyl substituent to from a hemiacetal, which decomposes to an unsaturated aldehyde and an alcohol. Upon the addition of a second equivalent of TBHP (to reoxidise the chromium catalyst), the resultant alcohol is then oxidised to the ketone. ${ }^{127}$ However when these conditions were applied to allyl ether $\mathbf{5 4}$, starting material was predominately isolated, scheme $37 .{ }^{1} \mathrm{H}$ NMR analysis of the crude 
material however, indicated the presence of trace amounts of an aldehyde moiety, suggesting that these reagents preferentially deprotected and oxidised the benzyl groups.



54



$\mathrm{CH}_{2} \mathrm{Cl}_{2}, \mathrm{rt}, 12 \mathrm{~h}$



55

Scheme 37: Attempted dual deprotection/oxidation of allyl ether $\mathbf{5 4}$

Allyl groups can also be oxidatively deprotected with chromia-pilliared montmorillonite clay and tert-butyl hydroperoxide. ${ }^{127,128}$ However due to mechanistic similarities between this system and the previous example, and the observation by Choudary et. al. that such systems can be extended successfully to oxidative debenzylation reactions, we opted against exploring this strategy and focussed on simply deprotecting the allyl group. Even though a dual deprotection/oxidation strategy may still be applicable for our system, this would involve a significant investment of time, and was not of utmost importance to our synthesis.

Ideally, a catalytic methodology was sought for the deprotection of the allyl group so the previous experimental conditions, using palladium on carbon to isomerise the double bond, ${ }^{118}$ were repeated on the correct substrate. Although this methodology looked somewhat promising, the reaction would not go to completion with extended reaction times resulting in the decomposition of the starting substrate. Consequently, the $\mathrm{PdCl}_{2}$, $\mathrm{CuCl}, \mathrm{O}_{2}$ system was investigated. Again initial results looked promising, but unfortunately similar difficulties to those observed with the $\mathrm{Pd} / \mathrm{C}$ system were encountered.

Although only a small proportion of the thirty or so methods for the deprotection of allyl groups $^{129}$ had been surveyed, an intuitive hunch prompted us to investigate the milder 
reaction conditions of palladium chloride, acetic acid, sodium acetate and water. ${ }^{124}$ Gratifyingly, the allyl ether was cleanly deprotected, forming the corresponding secondary alcohol $\mathbf{5 5}$ in a respectable $83 \%$ yield, scheme 38.



54



55

Scheme 38: Deprotection of the allyl group

This deprotection strategy however, is not without its pitfalls. Stoichiometric amounts of palladium chloride are required, which due to its expense, does not facilitate a particularly economic synthesis upon scale-up. Nevertheless, we decided to continue on with our strategy and experiment further with allyl deprotection methodologies at a later stage. Thus the final obstacle to overcome in this synthesis was the oxidation of the secondary alcohol.

Ideally, a fairly benign oxidation procedure was desirable, so investigations into the oxidation of alcohol $\mathbf{5 5}$ using an aqueous solution of sodium hypochlorite and acetic $\operatorname{acid}^{130}$ were conducted. Unfortunately, these conditions were not particularly effective for our system, with the desired ketone being formed in a mere $10 \%$ yield. The starting material predominantly decomposed, presumably as a consequence of over oxidation. Next, a TEMPO oxidation was investigated. This is also a fairly benign oxidation procedure, requiring only catalytic amounts of 4-methoxy-2,2,6,6,-tetramethylpiperidine1-oxyl with continuous generation of the oxoammonium salt by $\mathrm{NaOCl}$ under aqueous organic two-phase conditions. ${ }^{131}$ However, in contrast to the previous oxidation strategy, starting material was only ever isolated using this protocol.

Although not an ideal choice of reagent, we nevertheless turned towards the use of chromium derivatives as oxidising agents. Initially a solution of chromium trioxide in 
acetic acid looked promising, ${ }^{132}$ though the ketone was only ever obtained in a mere $30 \%$ yield. A solution of PCC and $\mathrm{CaCO}_{3}$ in dichloromethane, however, held more potential. Although the desired ketone 8 was synthesised in approximately $50 \%$ yield, the reaction would not go to completion, even if excess PCC and longer reaction times were used. Substitution of $\mathrm{CaCO}_{3}$ for $\mathrm{NaOAc}$ as the base offered some improvement, enabling ketone 8 to be synthesised in a $60 \%$ yield. Nevertheless, we were still plagued by incomplete reactions, with approximately $10 \%$ of the preceding alcohol still remaining.

A subsequent review of the literature however, indicated that such practical difficulties were frequently encountered. Unduly long reaction times and the requirement of a large excess of pyridinium chromate oxidising agents, leading to unsatisfactory yields, are common problems. ${ }^{133}$ Czernecki et $a l^{133 a}$ however, determined that the use of anhydrous acetic acid and freshly activated molecular sieve powder in combination with pyridinum dichromate (PDC) were the decisive factors for achieving reproducible and successful oxidations. Such adaptations enabled the amount of toxic PDC to be limited to 1.5 mole equivalents. Fortunately this methodology was also applicable for alcohol 55, with complete oxidation to the desired ketone 8 occurring in a respectable $84 \%$ yield, scheme 39.



Scheme 39: Synthesis of ketone 8

The ${ }^{1} \mathrm{H}$ NMR spectrum of ketone 8 is shown in figure 14 overleaf. The C-1 and C-4 protons, previously reported as doublet of doublets at $\delta 3.64$ and 3.49 , and as doublets at $\delta 3.38$ and $3.27 \mathrm{ppm}$, respectively, in alcohol $\mathbf{5 5}$ are now reduced to two singlets at $\delta 4.38$ and $\delta 3.44 \mathrm{ppm}$ in ketone $\mathbf{8}$. Similarly, the previously diastereotopic methyl substituents at $\delta 0.97$ and $0.96 \mathrm{ppm}$ in alcohol $\mathbf{5 5}$, are now homotopic with a chemical shift of 1.17 ppm in ketone $\mathbf{8}$. 




Figure 14: ${ }^{1} \mathrm{H}$ NMR of gem-dimethyl ketone 8 
Although it is highly plausible that another oxidation strategy may improve the yield or eliminate the need for a chromium reagent during the oxidation of $\mathbf{5 5}$, we were nevertheless satisfied with our methodology and the knowledge that the synthesis of the final gem-dimethyl ketone is plausible. All that remained was to reinvestigate alternative methods for the deprotection of the allyl group.

Unfortunately studies into the use of the $\mathrm{NaBH}_{4} / \mathrm{I}_{2}$ system ${ }^{121}$ and the $\mathrm{SeO}_{2}$ system $^{122}$ for the deprotection of allyl ether $\mathbf{5 4}$ were disappointing. Both resulted in the decomposition of the starting substrate. The use of Wilkinson's catalyst and DABCO (1,4-diazabicyclo[2.2.2] octane) in $10 \%$ aq ethanol initially looked more promising. ${ }^{120}$ Surprisingly though, analysis of the crude substrate by ${ }^{1} \mathrm{H}$ NMR indicated that there was a small amount of the debenzylated adduct along with the requisite deprotected allyl ether. This was unexpected as literature precedent indicated that benzyl groups are stable to these reaction conditions but the unwanted reaction may have occurred as a result of unduly long reaction times. ${ }^{\mathrm{IX}}$ An additional problem with this rhodium catalysed deprotection was the cleavage of the intermediate isomerised ether. We wished to avoid the $\mathrm{Hg}^{2+}$ promoted cleavage of the propenyl ether, and opted instead for an acidic workup, but this resulted in the incomplete cleavage of the isomerised ether. Although it appeared highly likely that these difficulties might be surpassed by careful manipulation of the reaction conditions, a fortuitous sequence of events prompted us to disregard this strategy.

A further review of the literature drew our attention to work published by Nagakura et. al. in 1997, whereby allyl ethers could be cleaved using $p$-toluenesulfinic acid as both an allyl acceptor and proton source in combination with catalytic amounts of $\mathrm{Pd}(0)$ in $\mathrm{CH}_{2} \mathrm{Cl}_{2} \cdot{ }^{134}$ As the sulfinic acid was not readily available, this methodology was not initially attempted. Simultaneously, a guest lecturer revealed that they had been

\footnotetext{
[x Significantly longer times than those reported in the literature were used as GC analysis indicated that the reaction was not complete
} 
having similar difficulties with the deprotection of allyl groups and found that with a slight modification of Nagakura's procedures, they were able to reproducibly deprotect allyl groups in good yields. A communication by Kunz and Opatz ${ }^{135}$ thus indicated that the choice of solvent played a critical role in this reaction with the use of 1,2dimethoxyethane or dioxane, as compared to dichloromethane, produced more reproducible results.

Thus the precedent of Kunz and Opatz was followed and attempts were made at deprotecting allyl ether $\mathbf{5 4}$ in dioxane with p-toluenesulfinic acid and catalytic (6 mol\%) $\mathrm{Pd}\left(\mathrm{PPh}_{3}\right)_{4}$; a slightly lower loading than that reported. Gratifyingly, a clean conversion of allyl ether $\mathbf{5 4}$ to alcohol $\mathbf{5 5}$ was observed. This initial result prompted further experimentations with catalytic loadings and solvents. Although additional reductions in the catalytic loading resulted in incomplete reactions, dioxane could be successfully substituted with the more environmentally benign and less hazardous THF, scheme 40 . Thus allyl ether $\mathbf{5 4}$ could be reproducibly deprotected with THF as the solvent.



54
$\mathrm{Pd}\left(\mathrm{PPh}_{3}\right)_{4}(6 \mathrm{~mol} \%)$ $p-\mathrm{TolSO}_{2} \mathrm{H}$

THF, rt, 7 h, $83 \%$<smiles>CC(C)(COCc1ccccc1)C(O)COCc1ccccc1</smiles>

55

Scheme 40: Catalytic deprotection of the allyl ether $\mathbf{5 4}$ with $\mathrm{Pd}\left(\mathrm{PPh}_{3}\right)_{4}$

The catalytic deprotection of the allyl ether $\mathbf{5 4}$ thus represents the last synthetic challenge in the synthesis of gem-dimethylketone $\mathbf{8}$. For clarity, the overall synthetic scheme is shown in scheme 41 overleaf, with the overall yield for this synthesis being a respectable $35 \%$ over the five steps. Furthermore, additional work in our laboratory has demonstrated that this strategy can be performed on the multi-gram scale. ${ }^{136}$ 
$\sum_{n+1} \mathrm{OH}$

17
1) $\mathrm{KO}^{\mathrm{t}} \mathrm{Bu}$

2) $\mathrm{Br}$ $81 \%$<smiles>C=CCOC1C(=O)OC[C@]1(C)C(F)(F)F</smiles>

$17 \mathrm{c}$



$\mathrm{HOH}_{\mathrm{H}}^{\mathrm{HOH}}$

53



Scheme 41: Overall synthesis of gem-dimethyl ketone $\mathbf{8}$ 


\section{Chapter Four}

\section{Synthesis of the C-12 to C-24 fragment of Peloruside A: Strategy 1}

\section{4:1: The lactone approach}

The first strategy investigated for the synthesis of the C-12 to C-22 fragment of pel A involved formation of lactone $\mathbf{2 0}$, scheme 42 . Lactone $\mathbf{2 0}$ is reductively opened, following a regio-and stereoselective aldol reaction with the pyranose fragment of pel A (depicted in red) to give the carbon skeleton below.


20

Scheme 42: Retrosynthetic analysis for the synthesis of the C-12 to C-24 fragment

To initially test the viability of this strategy, a racemic synthesis of the target lactone was investigated, with the ethyl substituent from the side chain also being omitted, scheme 43 . Modified lactone $\mathbf{5 6}$ results from the RCM of diene $\mathbf{5 7}$, itself formed from the esterification reaction of vinyl acetic acid $\mathbf{5 8}$ with the aldol adduct $\mathbf{2 4}$. With a view to investigating the bioactivity of analogues of pel $\mathrm{A}$, synthesis of the non-methylated $(\mathrm{R}=$ $\mathrm{H})$ analogue of pel A was also explored 




56<smiles>C#CCC(OC(=O)CC=C)C(=C)P</smiles>

57<smiles>[R]C(=C)C(O)CC(C)=O</smiles>

58

Scheme 43: Retrosynthetic analysis for the synthesis of the model C-12 to C-24 fragment

The starting point in this synthesis involved formation of the $\beta$-hydroxyketones, 24a and 24b, using standard, low temperature aldol reactions, scheme 44. Literature precedent was followed for the synthesis of ( \pm )-4-hydroxy-5-hexene-2-one $\mathbf{2 4 a}$, whereby the lithium enolate of acetone was captured by acrolein. ${ }^{137}$ A slight adaptation of this protocol, with methacrolein being substituted for acrolein, enabled us to synthesise the methylated derivative $\mathbf{2 4 b}$, in $84 \%$. The successful reproduction of these results however depended greatly upon the purity and dryness of the solvents and reagents, particularly that of the THF. Inadequately dry THF often resulted in lower yields and a propensity of the resulting $\beta$-hydroxy ketone to undergo elimination, forming the highly unsaturated dienone $\mathbf{5 9}$.
<smiles>C=C(P)C(O)CC(C)=O</smiles>

$24 \mathrm{a} R=\mathrm{H}(80 \%)$

$24 b \mathrm{R}=\mathrm{Me}(84 \%)$



59

Scheme 44: Formation of ß-hydroxyketones $24 \mathrm{a}$ and $24 \mathrm{~b}$

The $\beta$-hydroxyketone was then coupled to vinyl acetic acid following a standard dicyclohexylcarbodiimide (DCC), dimethylaminopyridine (DMAP) esterification protocol. Although this reaction successfully produced the corresponding esters, 57a and $\mathbf{5 7 b}$, scheme 45 , purification proved to be somewhat tedious. Flash chromatography would not adequately remove all of the dicyclohexyl urea by-product formed during this 
reaction without severely jeopardising the yield, yet bulb-to-bulb distillation alone would not separate the small portion of the $\alpha, \beta$ unsaturated dienone $\mathbf{5 9}$ also formed during the reaction. Consequently the most successful purification strategy involved a combination of flash chromatography, followed by bulb-to-bulb distillation. Although this was not ideal methodology, yields of $80 \%$ or greater were nevertheless obtained for the synthesis of esters $\mathbf{5 7} \mathbf{a}$ and $\mathbf{5 7} \mathbf{b}$.



$24 a \mathrm{R}=\mathrm{H}$

24b $R=M e$



$0^{\circ} \mathrm{C}-\mathrm{RT}, 14 \mathrm{~h}$

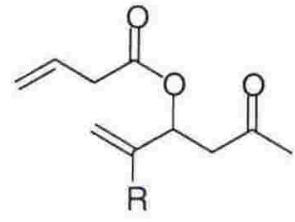

$57 a \mathrm{R}=\mathrm{H}, 84 \%$

$57 b \mathrm{R}=\mathrm{Me}, 80 \%$

Scheme 45: Synthesis of esters $\mathbf{5 7 a}$ and $\mathbf{5 7 b}$

Due to these difficulties, alternative coupling reagents were investigated. Although the use of 1-[3-(Dimethylamino)propyl]-3-ethylcarbodiimide hydrochloride (EDCI) resulted in the formation of the desired ester, a significant portion of the conjugated enone $\mathbf{5 9}$ resulted, even after formation of a pre-mixed solution of vinyl acetic acid and EDCI at $0{ }^{\circ} \mathrm{C}$. The alternative choice of carbonyldiimidazole (CDI) as a coupling reagent resulted in an uncharacterisable mixture of products.

Nevertheless, with the esters $\mathbf{5 7} \mathbf{a}$ and $\mathbf{5 7} \mathbf{b}$ in hand, the next stage in the strategy involved the synthesis of the corresponding lactones via RCM. Some of the more common alkylidene-metal complexes widely used for RCM include the alkoxy-imido molybdenum complex (the Schrock/Hoyveda catalyst) $\mathbf{6 0},{ }^{138}$ the benzylidene ruthenium complex (Grubbs' Catalyst) $\mathbf{6 1}^{139}$ and the modified benzylidene ruthenium complexes coordinated to 1,3-dimesitylimidazol-2-ylidene ligands (Grubbs' second generation


appropriate catalyst for our substrate were reactivity, stability and availability/cost. 




60



61



62

Figure 15: Examples of common metal-alkylidene metathesis catalysts

Compared to Grubbs' catalyst $\mathbf{6 1}$, the molybdenum complex $\mathbf{6 0}$ exhibits high reactivity towards a broad range of substrates containing numerous steric and electronic variations. ${ }^{140}$ Unfortunately though, it also suffers from extreme sensitivity to air and moisture as well as decomposition upon storage. Grubbs' second-generation catalyst $\mathbf{6 2}$ however, exhibits activity similar to that of the molybdenum complex $\mathbf{6 0}$ yet has also retained remarkable air and moisture stability characteristic of the parent benzylidene ruthenium complex 61. ${ }^{141}$ The enhanced reactivity of Grubb's second-generation ruthenium catalyst over its first generation counterpart is believed to arise as a result of the greater basicity of the imidazole ligands, when compared to their unsaturated analogues, which in turn translates into increased activity. ${ }^{142}$ This enhanced basicity is due to the lack of carbene stabilisation provided by the absence of $\pi$-interactions.

Although one would thus expect the second-generation ruthenium catalyst $\mathbf{6 2}$ to be the catalyst of choice, due to its expense (or the additional steps required for its synthesis) ${ }^{141}$ we opted to first explore the viability of the commercially available Grubbs' first generation ruthenium catalyst $\mathbf{6 1}$. This catalyst generally exhibits excellent activity for ring closing metathesis resulting in the formation of five or six-membered rings, although its activity drops dramatically for the synthesis of larger cyclic compounds. ${ }^{140}$ Difficulties in the RCM of substituted olefins using Grubbs' first generation catalyst have also been reported. ${ }^{140,141}$ 
As the synthesis of lactone 56a required RCM involving an unsubstitued olefin, we were fairly confident that Grubbs' first generation catalyst would be adequate for this purpose. Accordingly, we were justly rewarded when lactone 56a was synthesised in a $90 \%$ yield, scheme 46. One disadvantage of this strategy however, was that $5 \mathrm{~mol}$ percent of the catalyst was required for complete cyclisation. Several attempts were made at decreasing the catalytic loading, but without success. As a consequence, purification of the lactone proved to be somewhat difficult and tedious. ${ }^{x}$ Separation of the residual ruthenium from the desired product by flash chromatography was problematic, and bulb-to-bulb distillation simply resulted in decomposition of lactone.



Scheme 46: Synthesis of lactone 56a

An interesting observation however was made upon the attempted cyclisation of ester $\mathbf{5 7 b}$, scheme 47 . The ${ }^{1} \mathrm{H}$ NMR of the product, after a solution of ester $\mathbf{5 7 \mathbf { b }}$ was refluxed with five mol \% of 61 in $\mathrm{CH}_{2} \mathrm{Cl}_{2}$ for 24 hours, revealed some anomalous peaks, particularly in the olefinic region of the spectrum. Two singlets were observed at $\delta 4.98$ and $\delta 4.90 \mathrm{ppm}$, integrating for two protons each, along with two multiplets at $\delta 5.76$ and $\delta 5.68 \mathrm{ppm}$. Additionally, the ${ }^{13} \mathrm{C}$ NMR of this sample revealed thirteen ${ }^{13} \mathrm{C}$ resonances, four more than that of the anticipated lactone $\mathbf{5 6 b}$.<smiles>C=CCC(=O)OC(CC(C)=O)C(=C)C</smiles>

$57 \mathrm{~b}$

<smiles>CCC(=O)CC1OC(=O)CC=C1C</smiles>

$56 b$

Figure 47: Attempted RCM of ester 57b using Grubbs' catalyst 61

\footnotetext{
${ }^{x} 5$ mol\% of the catalyst equates to approximately $25 \mathrm{wt} \%$
} 

product still prevailed. Clearly the methyl substituent on the olefin was hindering intramolecular cyclization.

Given this observation, it was concluded that a more active metathesis catalyst was required for the RCM of the methylated substrate. Due to the availability of materials, studies into the applicability of the ruthenium vinylidene metathesis catalyst $\mathbf{6 4}$ generated in situ (scheme 49), ${ }^{143}$ were initially explored. Although reaction rates are slightly slower for this vinylidene catalyst, its general reactivity profile towards a variety of substrates is similar to the analogous, highly active, second generation ruthenium alkylidene compounds.



$-\mathrm{HO}^{\mathrm{t} B u}$

$$
\left[\left\{(\text { p-cymene }) \mathrm{RuCl}_{2}\right\}_{2}\right]
$$



$$
\mathrm{H}={ }_{-\mathrm{Bu}}^{\mathrm{H}} \mathrm{B}
$$


Scheme 49: Proposed pathways for catalyst generation and decomposition ${ }^{143}$ 
As depicted in scheme 49 , the formation of the ruthenium vinylidene catalyst requires the use of one equivalent of sodium tert-butoxide. Unfortunately the presence of this base during the attempted RCM of $\mathbf{5 7}$ resulted in alkene isomerization. The analogous $\alpha, \beta$ unsaturated ester was the only product isolated. Initially it was proposed that an increase in the length of time between the addition of sodium tert-butoxide and diene $\mathbf{5 7}$ may ensure complete deprotonation of the imidazolinylidene ligand, and hence, prevent the excess $\mathrm{NaO}^{\prime} \mathrm{Bu}$ from causing the unwanted isomerization. However, during these studies we were able to obtain Grubbs' second-generation ruthenium benzylidene catalyst $\mathbf{6 2}$. Thus we opted to investigate the potential of 62 in the RCM of diene $57 \mathbf{b}$ and discontinued the studies involving the in situ formation of the vinylidene catalyst.

Fortunately the greater reactivity of second-generation ruthenium benzylidene catalyst $\mathbf{6 2}$ enabled the desired lactone $\mathbf{5 6} \mathbf{b}$ to be synthesised in an $85 \%$ yield, scheme 50 . The ${ }^{13} \mathrm{C}$ NMR spectrum of lactone $\mathbf{5 6} \mathbf{b}$ is shown in figure 16 overleaf; with the signals for the C-8 and C-9 methyl carbons at $\delta 30.2$ and $\delta 18.8$ ppm, signals for the C-2 and C-6 methylene carbons at $\delta 46.8$ and $\delta 31.3 \mathrm{ppm}$, the signal for the C-5 methine carbon at $\delta 78.9 \mathrm{ppm}$, the signal for the $\mathrm{C}-3$ olefinic methylene carbon at $\delta 117.6 \mathrm{ppm}$, with the remaining quarternary olefinic, ester and ketone carbons signals at $\delta 131.9, \delta 169.0$ and $\delta 204.8$ ppm, respectively. Moreover, the RCM of diene $57 \mathrm{~b}$ was conducted with only a $1.5 \%$ catalytic loading. Given these positive results, the ruthenium benzylidene catalyst $\mathbf{6 2}$ was used for the RCM of the unsubstituted diene 57a. The lower catalytic loading would alleviate some of the problems with the purification of the resulting lactone. Indeed, lactone 56a was once again synthesised in a $90 \%$ yield, but this time only a $1 \%$ catalytic loading was required.




Scheme 50: RCM using second-generation Grubbs' catalyst 62


Figure 16: ${ }^{13} \mathrm{C}$ NMR of lactone 56b

With the key intermediate lactones synthesised, all that remained in this strategy was a regioselective aldol reaction between the lactone and the pyranose ring of peloruside. Even though there is a multitude of precedent for the selective formation of kinetic verses thermodynamic enolates, our system presented additional difficulties with the possibility of both double bond isomerization and a second aldol reaction occurring $\alpha$ or $\gamma$ to the lactone carbonyl. Thus, to investigate this system, aldol reactions were conducted with butyraldehyde or benzaldehyde being used as model substrates for the pyranose ring, scheme 51 . 
<smiles>[R]C1=CCC(=O)OC1CC(C)=O</smiles>

56a $\mathrm{R}=\mathrm{H}$

$56 \mathrm{~b} R=\mathrm{Me}$
1) LDA or LTMP, $-78^{\circ} \mathrm{C}$

2) $\mathrm{R}^{2} \mathrm{CHO}$<smiles>[R]C1=CCC(=O)OC1CC(=O)CC([R])O</smiles>

$\mathrm{R}=\mathrm{H}, \mathrm{Me}$

$\mathrm{R}^{2}=\mathrm{Ph}, \mathrm{C}_{3} \mathrm{H}_{7}$

Scheme 51: Model aldol studies with lactones 56a and 56b

Initial studies involved enolate formation at $-78{ }^{\circ} \mathrm{C}$ using $\mathrm{LDA}$ as the base. Unfortunately, these reactions were problematic. The desired aldol product was formed along with a multitude of other products; as indicated by ${ }^{1} \mathrm{H}$ NMR and HRESIMS analysis. Furthermore there was evidence for an aldol reaction occurring $\alpha$ to the lactone. Consequently it was postulated that a more sterically hindered base, such as LTMP, would favour the formation of the kinetic enolate at $-78{ }^{\circ} \mathrm{C}$. Again, numerous aldol reaction studies were conducted with LTMP as the base, but once again, mixtures of products resulted. Although disappointing, these results were not entirely unexpected.

The pKa values of ester and ketone $\alpha$ protons are approximately 25 and 20, respectively. Thus one would expect the more acidic ketone $\alpha$-proton (C-8) of $\mathbf{5 6}$ to deprotonate preferentially. Incorporation of the adjacent olefin moiety in lactone $\mathbf{5 6}$ however, would result in a decrease in the pKa value of the lactone $\alpha$-proton (C-2) in 56. At the time of commencing the synthesis, we were unsure about the influence that the olefinic moiety would have on the pKa of the lactone $\alpha$-proton. However from the above experimental results, the pKa at C-2 appears to be equivalent to, or perhaps even lower, than that at C8 , as a mixture of aldol products were obtained.

Subsequently, a pKa prediction server called SPARC ${ }^{\mathrm{X} 1}$ was used to predict the pKa values of the $\alpha$ protons at the methylene C-2 and methyl C-8 positions of 56 . Remarkably, a pKa of 11.5 was reported for the C-2 position, with a value of 17.3 being 
given for $\mathrm{C}$ - 8 . The dramatic decrease in the $\mathrm{pKa}$ of the lactone $\alpha$-proton was somewhat unexpected.

Potentially the problems in controlling the regiochemisty of the aldol reactions could be circumvented by protecting the ketone under neutral conditions using 1,2-di(trimethylsilyoxy)ethane, scheme 52. Reduction of the lactone, followed by protection of the resulting diol, would then enable a regioselective, kintetically controlled, aldol reaction to be preformed at the desired $\alpha$-methyl position. However, this modification adds several steps to our synthesis and is, for the most part, undesirable.<smiles>[R]C1=CC([R])C(=O)OC1CC(C)=O</smiles>

$56 \mathrm{a} \mathrm{R}=\mathrm{H}$ $56 \mathrm{~b} R=\mathrm{Me}$



TMSOTf



1) $\mathrm{LiAH}_{4}$

2) Protection



Scheme 52: Alternative strategy for the synthesis of the C-12 to C-24 fragment of peloruside $\mathrm{A}$

Alternatively, one may speculate that the combination of the ethyl substituent, where $\mathrm{R}^{2}=$ Et, and a sterically hindered base such as LTMP, may prevent the lactone $\alpha$-proton from being deprotonated. Although a plausible suggestion, we believed that enough time had been spent on this strategy and an alternative, less problematic, methodology should be investigated. Consequently we discontinued these studies and turned our attention towards the synthesis of the $\mathrm{C}-12$ to $\mathrm{C}-24$ fragment using the cyclic bis silyl ether tether.

\footnotetext{
${ }^{\mathrm{xI}}$ See, http://ibmlc2.chem.uga.edu/sparc/index.cfm
} 


\section{Chapter Five}

\section{Synthesis of the C-12 to C-24 fragment of peloruside A: Strategy Two}

\section{1: Initial aldol studies with the cyclic bis-silyl ethers}

As the previously described strategy for the synthesis of the C-12 to C-24 fragment of pel A was problematic, an alternative strategy involving the synthesis of the bis-alkoxysilyl ether 22 was investigated, scheme 53. It was anticipated that RCM of the appropriate acyclic precursor 23 would form the desired bis-silyl ether $\mathbf{2 2}$. Diene $\mathbf{2 3}$ could in turn, be synthesised by the addition of $\beta$-hydroxyketone 24 and ( $S$ )-2-ethyl-but3-ene-1-ol 25 to the appropriate dichlorosilane. In this way, the silane acts as both a tether for the RCM and a protecting group for both alcohols.



22



23





24



25

Scheme 53: Retrosynthetic stragety (II) for synthesis of the C-12 to C-24 fragment of peloruside A

There were many factors that needed to be considered when choosing the appropriate dichlorodialkylsilyl substrate as a tether for this reaction. First, the bis-silyl ether 22 needed to be robust enough to withstand the aldol reaction and subsequent chemical transformations. Second, the intermediate acyclic silyl tethered ether $\mathbf{2 3}$ needed to be amenable to RCM and finally, the impact of the silyl substituents $(\mathrm{R})$ in the subsequent asymmetric aldol reactions needed to be addressed. Although it was anticipated that the 
larger phenyl substituents would form a more robust cyclic ether and may offer advantages in controlling the subsequent asymmetric aldol reaction (see chapter 6), many of the reported RCM studies forming similar silyl tethered ethers had been conducted with the smaller methyl substituents. ${ }^{89,90,144}$ Even though many disubstituted isopropyl and phenyl derivatives had been synthesised for other purposes, ${ }^{145}$ it was unknown if the steric, or electronic constraints of these larger substituents would impede the RCM reaction of our substrate. ${ }^{146}$ In view of these considerations, all three alkyl groups were initially investigated in order to find the combination of reagents that would best suit our purposes.

The above retrosynthetic strategy was further modified in that although both $(R)$ - and $(S)$ 2-ethylbut-3-en-1-ol are available in one step using a $\mathrm{Zr}$-catalysed ethylmagnesation of 2,5-dihydrofuran, ${ }^{147}$ for simplicity but-3-en-1-ol was coupled to the appropriate silyl derivative, scheme 54. Our emphasis was initially on establishing a racemic synthesis for the C-12 to C-24 fragment of pel A. Once a plausible racemic strategy had been devised, this would then be adapted for the asymmetric synthesis of peloruside A.



65





66

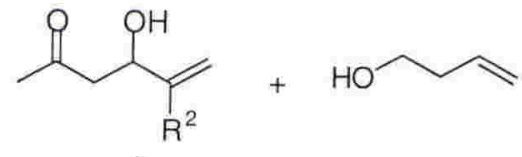

24a $R^{2}=H$ $24 b R^{2}=M e$

Scheme 54: Simplified retrosynthetic analysis for synthesis of the C-12 to C-24 fragment

Thus, the first step in this strategy was the formation of the mono silyl ethers $\mathbf{6 8}$, scheme 55. During these syntheses, problems were encountered in preventing disubstitution from occurring. Even though numerous changes were made to the reaction conditions in an attempt to improve the ratio of mono- to disubstitution adducts, the controlling factor in determining whether mono- or disubstitution occurred appeared to be the size of the substituents on the silyl tether, Disubstitution predominates with small 
substituents when the solution of but-3-en-1-ol in dichloromethane was slowly added, at $0{ }^{\circ} \mathrm{C}$, to a solution of the dichlorosilane in dichloromethane. For example, with dimethyldichlorosilane as a starting substrate, the desired monosilyl ether, but-3-en-1oxydimethylsilyl chloride 68c, was formed in a mere $17 \%$ yield. The undesired diether was formed in $50 \%$ yield. These results progressively improved as the size of the alkyl group increased, with isopropyl $\mathbf{6 8} \mathbf{b}$ giving the desired monosilyl ether in $50 \%$ yield, and the diphenyl substrate 68a giving the analogous diphenyl monosilyl ether in approximately ${ }^{x I I} 75 \%$ yield. No improvement could be made with these yields, even when the temperature of the initial reaction mixture was lowered to $-40{ }^{\circ} \mathrm{C}$. Additionally, the reaction would not go to completion unless the solution was refluxed.


Scheme 55: Synthesis of the but-3-en-1-oxydialkylsilyl chlorides

The B-hydroxy ketone $\mathbf{2 4}$ was then coupled to the silyl tethers $\mathbf{6 8}$ to form the corresponding bis-silyl ether adducts 66, scheme 56. As but-3-en-1-oxydiphenylsilyl chloride 68 a could not be isolated by fractional distillation due to its high boiling point, this substrate was made in situ and the corresponding B-hydroxy ketone added to the reaction vessel. Yields for the synthesis of the but-3-en-1-oxydialkylsilyl chloride derivatives are summarised in Table 1.

\footnotetext{
${ }^{\text {XII }}$ But-3-en-1-oxydiphenylsilyl chloride could not be purified by fractional distillation. The percentage of mono-to di-substituted adduct was based upon ${ }^{1} \mathrm{H}$ NMR analysis of the crude substrate.
} 




68



66

Scheme 56: Synthesis of the but-3-en-1-oxydialkylsilyl chloride derivatives 66

Table 1. Yields for the formation of the but-3-en-1-oxydialkylsilyl chloride derivatives

\begin{tabular}{clll}
\hline Entry & Compound & Substituents & Isolated yield \\
\hline 1 & $\mathbf{6 6 a}$ & $\mathrm{R}^{1}=\mathrm{Ph}, \mathrm{R}^{2}=\mathrm{Me}$ & $61 \%^{\mathrm{a}}$ \\
2 & $\mathbf{6 6 b}$ & $\mathrm{R}^{1}=\mathrm{Ph}, \mathrm{R}^{2}=\mathrm{H}$ & $65 \%^{\mathrm{a}}$ \\
3 & $\mathbf{6 6 c}$ & $\mathrm{R}^{1}={ }^{\mathrm{i}} \mathrm{Pr}, \mathrm{R}^{2}=\mathrm{Me}$ & $86 \%^{\mathrm{b}}$ \\
4 & $\mathbf{6 6 d}$ & $\mathrm{R}^{1}={ }^{\mathrm{i}} \mathrm{Pr}, \mathrm{R}^{2}=\mathrm{H}$ & $82 \%^{\mathrm{b}}$ \\
5 & $\mathbf{6 6 e}$ & $\mathrm{R}^{1}=\mathrm{Me}, \mathrm{R}^{2}=\mathrm{H}$ & $77 \%$ \\
\hline
\end{tabular}

averall yield from $\mathrm{Ph}_{2} \mathrm{SiCl}_{2} ;{ }^{\mathrm{b}}$ Catalytic DMAP required

Although formation of these silyl adducts occurred relatively smoothly and in good yields, it was interesting to note that formation of the butenoxydiisopropylsilyl chloride derivatives, $\mathbf{6 6} \mathbf{c}$ and $\mathbf{6 6} \mathbf{d}$, required the addition of catalytic amounts of DMAP. DMAP displaces the chloride ion, forming the silyl pyridinium ion, which is more amenable to nucleophilic displacement by the approaching alcohol. ${ }^{148}$ DMAP however, is not required for the analogous but-3-en-1-oxydimethylsilyl chloride derivative 66e. Because of the smaller size of the methyl substituents, the approaching alcohol can more readily approach the silicon atom and hence displace the chloride ion in the dimethyl tethered adducts. Even though the diphenyl substituents of the analogous but-3-en-1oxydiphenylsilyl chloride derivatives, $66 \mathrm{a}$ and $\mathbf{6 6} \mathrm{b}$, are similar in size to the isopropyl substituents, presumably the electron donating properties of the phenyl substituents favour the displacement of the chloride ion, making it act as a better leaving group. Thus, this facilitates the nucleophilic displacement by the approaching alcohol. 
In an attempt improve the initial selectivity of the first mono etherification reaction the order of addition of the two alcohols was reversed. By adding the more sterically encumbered $\beta$-hydroxyketone $\mathbf{2 4}$ first we anticipated that this would limit the amount of the disubstituted adduct formed, scheme 57. However, changing the order of addition simply resulted in elimination of a hydroxide ion from the $\beta$-hydroxyketone to form the unsaturated ketone 59. Presumably this elimination occurs via an $E 1_{c b}$ mechanism involving deprotonation of $\mathbf{2 4}$ to form the thermodynamically stable, highly conjugated enone 59. The electron withdrawing chlorides on the dichlorosilane derivatives 67 undoubtedly make these substrates less amenable to alkoxy substitution reactions with the competing $E 1_{\mathrm{cb}}$ elimination being favoured. A variety of conditions were trialed, however in all instances, the unsaturated ketone $\mathbf{5 9}$ predominated.



67



$\mathrm{O}^{\circ} \mathrm{C}-\mathrm{rt}$



69



Scheme 57: Attempted reversal of the order of addition of the hydroxy substrates

Nevertheless, with the required bis silyl ethers 66a-e in hand, investigations into the corresponding RCM reactions were conducted, scheme 58. As Boiteau et. al., ${ }^{149}$ had used Grubbs' second generation ruthenium catalyst $\mathbf{6 2}$ in refluxing benzene for the RCM of similar silyl tethered substrates, these conditions were initally used for the RCM of 66e. Although the desired cyclic ether was formed, an unknown, and inseparable byproduct was also formed in almost equivalent yield. The solvent was then changed to dichloromethane and the solution was heated to reflux with five mole percent of $\mathbf{6 2}$. T.l.c analysis of the reaction solution after 30 minutes however, indicated that at least three compounds were now present. Clearly an impurity was forming at a rate similar to that of the desired cyclic ether. The reaction was then conducted in $\mathrm{CH}_{2} \mathrm{Cl}_{2}$ at room temperature, again with $5 \mathrm{~mol} \%$ of the catalyst. Although the side product was once again formed, 
this time it was far less prevalent. However $5 \mathrm{~mol} \%$ of $\mathbf{6 2}$ was evidently not sufficient for this reaction as the starting acyclic silyl ether was still present.



Scheme 58: Desired RCM of the but-3-en-1-oxydialkylsilyl chloride derivatives

The instability of the dimethyl silyl tether was potentially the cause of the impurity, therefore the RCM of the diphenyl tethered substrate $\mathbf{6 6} \mathbf{b}$, was attempted. It was also unknown if this diphenyl tethered substrate would even be amenable to RCM. Seven mole \% of catalyst $\mathbf{6 2}$ was thus added to a solution of $\mathbf{6 6} \mathbf{b}$ in $\mathrm{CH}_{2} \mathrm{Cl}_{2}$ and the reaction stirred at room temperature for 10 hours. Fortunately, the desired cyclic adduct was formed, but again a small amount of an impurity was present. These results however did indicate that the RCM of the bis-silyl ether is a finely tuned process depending on both the reaction temperature and catalytic loading and not on the alkyl or aryl substituents on the silyl tethers. Thus, by carefully deducing the optimum amount of catalyst required for each system, one should be able to preferentially form the desired cyclic adduct and control undesirable competing reactions.

This assumption was investigated by repeating the $\mathrm{RCM}$ of $\mathbf{6 6} \mathbf{b}$ in $\mathrm{CH}_{2} \mathrm{Cl}_{2}$ at room temperature with a $10 \%$ catalytic loading of 62 . Fortunately, the desired cyclic silyl ether was formed almost exclusively. The optimum conditions for the RCM of the isopropyl tethered silyl ether $\mathbf{6 6} \mathbf{d}$, where $\mathrm{R}^{2}=\mathrm{H}$, also required the addition of at least 10 mole percent of $\mathbf{6 2}$, with the desired 4,8-O-diphenylsilanediyloct-5-en-2-one being formed in a respectable $81 \%$. Due to the very poor yield (17\%) when forming the initial dimethylsilyl chloride adduct, it was decided that the dimethylsilyl tether was an inappropriate reagent to use synthetically and the synthesis 4,8-di-O-dimethylsilanediyl-5-octen-2-one was never optimised. 
The RCM of the acyclic ethers where $\mathrm{R}^{2}=\mathrm{Me}$ however, proved to be a little more problematic. Due to the increased steric requirement of the methyl substituented olefin, and hence slower reaction rates, a minimum of 12 mole percent of the catalyst was required for these RCM reactions. Yields for these cyclisations were also slightly lower at $54 \%$ for the phenyl derivative and $60 \%$ for the isopropyl derivative. A summary of the RCM of the bis-silyl ether precursors is given in table 2.

Table 2. Synthesis of bis-silyl cyclic ethers 65 by RCM

\begin{tabular}{clll}
\hline Entry & Compound & Substituents & Isolated yield \\
\hline 6 & $\mathbf{6 5 a}$ & $\mathrm{R}^{1}=\mathrm{Ph}, \mathrm{R}^{2}=\mathrm{Me}$ & $54 \%$ \\
7 & $\mathbf{6 5 b}$ & $\mathrm{R}^{1}=\mathrm{Ph}, \mathrm{R}^{2}=\mathrm{H}$ & $81 \%$ \\
8 & $\mathbf{6 5 c}$ & $\mathrm{R}^{1}=i \mathrm{Pr}, \mathrm{R}^{2}=\mathrm{Me}$ & $60 \%$ \\
9 & $\mathbf{6 5 d}$ & $\mathrm{R}^{1}=i \mathrm{Pr}, \mathrm{R}^{2}=\mathrm{H}$ & $74 \%$ \\
10 & $\mathbf{6 5 e}$ & $\mathrm{R}^{1}=\mathrm{Me}, \mathrm{R}^{2}=\mathrm{H}$ & $42 \%$ \\
\hline
\end{tabular}

"Due to the low yields of the proceeding monosilyation reaction, RCM was not optimised

The ${ }^{1} \mathrm{H}$ NMR spectrum of 4,8-O-diphenylsilanediyl-5-methyloct-5-en-2-one $\mathbf{6 5 a}$ is given in figure 17 overleaf. The three dimensional representation of $\mathbf{6 5} \mathbf{a}$ is shown as one enantiomer in figure 18 for ease of discussion. Noteworthy features in the ${ }^{1} \mathrm{H}$ spectrum include the differentiation of the diastereotopic protons on C-3 with signals at $\delta 2.94$ (dd, $J=9.9,15.3 \mathrm{~Hz})$ and $\delta 2.62(\mathrm{dd}, J=3.6,15.3 \mathrm{~Hz})$. The large trans coupling of $9.9 \mathrm{~Hz}$ between $\mathrm{H}-4$ and H-3b enables us to assign the signal for $\mathrm{H}-3 \mathrm{~b}$ at $\delta 2.94$, and 3 a therefore at $\delta$ 2.62. Ring protons $\mathrm{H}-7 \mathrm{a}$ and $\mathrm{H}-7 \mathrm{~b}$ are also in quite distinct chemical environments with signals at $\delta 2.93$ and $\delta 2.26$, respectively. As illustrated in figure 18 , the shielding effect of the ring oxygens on $\mathrm{H}-7 \mathrm{~b}$ accounts for its high field shift. A summary of the ${ }^{1} \mathrm{H}$ and ${ }^{13} \mathrm{C}$ NMR spectral assignments for $65 \mathbf{a}$ is given in table 3 . 



Figure 17: 'H NMR spectrum of 4,8-O-diphenylsilanediyl-5-methyloct-5-en-2-one 65a 



Figure 18: 4,8-O-diphenylsilanediyl-5-methyloct-5-en-2-one 65a

Table 3: ${ }^{1} \mathrm{H}$ and ${ }^{13} \mathrm{C}$ NMR data for $65 \mathrm{a}$

\begin{tabular}{lll}
\hline Position & $\boldsymbol{\delta}^{\mathbf{1 3} \mathbf{C}}$ & $\boldsymbol{\delta}^{\mathbf{1}} \mathbf{H}$ \\
\hline 1 & 30.9 & $2.25(\mathrm{~s}, 3 \mathrm{H})$ \\
2 & 207.6 & - \\
$3 \mathrm{a}$ & 49.6 & $2.62(\mathrm{dd}, J=3.6,15.3 \mathrm{~Hz}, 1 \mathrm{H})$ \\
$3 \mathrm{~b}$ & - & $2.94(\mathrm{dd}, J=9.9,15.3 \mathrm{~Hz}, 1 \mathrm{H})$ \\
4 & 71.7 & $4.90(\mathrm{dd}, J=3.6,9.9 \mathrm{~Hz}, 1 \mathrm{H})$ \\
5 & 140.8 & - \\
6 & 125.1 & $5.44(\mathrm{t}, J=8.1 \mathrm{~Hz}, 1 \mathrm{H})$ \\
$7 \mathrm{a}$ & 31.2 & $2.93(\mathrm{~m}, 1 \mathrm{H})$ \\
$7 \mathrm{~b}$ & - & $2.26(\mathrm{~m}, 1 \mathrm{H})$ \\
8 & 64.4 & $4.05(\mathrm{~m}, 2 \mathrm{H})$ \\
$9(\mathrm{Me})$ & 21.5 & $1.60(\mathrm{~s}, 3 \mathrm{H})$ \\
$10 \mathrm{a}$ & 133.7 & - \\
$10 \mathrm{~b}$ & 133.4 & - \\
11 & 134.8 & $7.64(\mathrm{~m}, 4 \mathrm{H})$ \\
12 & 128.1 & $7.39(\mathrm{~m}, 6 \mathrm{H})$ \\
13 & 130.4 & $7.39(\mathrm{~m}, 6 \mathrm{H})$ \\
\hline & &
\end{tabular}


With a plausible strategy for the synthesis of the requisite cyclic ethers in place, model aldol studies were conducted, scheme 59. Initially diphenylsilane $\mathbf{6 5} \mathbf{b}$ was chosen as the starting ketone, and was subjected to a LDA mediated aldol reaction, using butyraldehyde as the aldehyde acceptor. Although the absence of the methyl singlet (formerly at $\delta 2.22$ ) in the ${ }^{1} \mathrm{H}$ NMR of the crude reaction product indicated that an aldol reaction had occurred, the reaction had not proceeded in the desired manner. Not only had a menagerie of products been formed but it also appeared that the silyl tether had been partially cleaved. TLC analysis of the crude product mixture indicated that many polar products had been formed. Similar results were observed for the 4,8-Odiphenylsilanediyl-5-methyloct-5-en-2-one 65a and 4,8-O-dimethylsilanediyloct-5-en-2one 65e derivatives. Unfortunately, attempts made at deciphering the products were unsuccessful due to the vast assortment of products formed.



65
1) LDA or LTMP, $-78^{\circ} \mathrm{C}$

2)<smiles>[B]C=O</smiles>



Scheme 59: Model aldol reactions with the bis-silyl ethers

Even though the aforementioned reactions were conducted under kinetic conditions $\left(-78^{\circ} \mathrm{C}\right)$, it was postulated that potentially excess base had deprotonated an $\alpha$-methylene proton resulting in elimination of the siloxy group via an $\mathrm{E} 1_{\mathrm{cb}}$ type mechanism. A series of singlet peaks at approximately $\delta 7.3,7.2,6.3$ and $6.2 \mathrm{ppm}$ were observed in the ${ }^{1} \mathrm{H}$ NMR spectra for the partically purified products, providing some evidence for the formation of an unsaturated dienone analogous to 59. Thus, the above aldol reaction was repeated, this time using LTMP, a more sterically hindered base. Extreme care was also taken to ensure that no excess base was present. However, similar results were observed. A change in aldehyde acceptor from butylaldehyde to pivaldehyde did not improve the outcome. Again the aldol reaction was repeated, but this time the aprotic solvent 
hexamethylphosphoramide (HMPA) was added in an attempt to solvate the lithium ion, and hence, assist in the formation of the enolate. Unfortunately, once again similar results were observed. It appeared as if an aldol reaction had occurred, yet the silyl tether was simultaneously being cleaved. Due to the vast assortment of products, it was never possible to isolate any single adduct to confirm these propositions.

These results were very frustrating as the silyl tether appeared to simply be not robust enough for these particular aldol reactions. This was a particularly puzzling observation. Although we were unaware of any literature procedures for the aldol reactions of similar cyclic silyl tethered substrates, analogous substrates had been subjected to demanding reaction conditions. For example, a seven membered bis cyclic ether had been synthesised by the $\mathrm{Fe}(\mathrm{CO})_{5}$-promoted cyclocarbonylation of a diyne in toluene at $120^{\circ} \mathrm{C}$ for 24 hours. ${ }^{150}$ However, based upon the above observations, one could only conclude that a more robust tether was required.

An alternative silyl tether that may be a slightly more robust was the di-tert-butyl silyl derivative. Without any ready access to di-tert-butyldichlorosilane, in a somewhat unprecedented manner, attempts were made at forming the acyclic bis-ethers using ditert-butylsilylbis(trifluoromethanesulfonate) $\mathbf{7 0}$ as a starting substrate. ${ }^{\mathrm{XIII}}$ To ascertain if this methodology was feasible, a two-step, one-pot reaction was conducted whereby a solution of 70 in DMF at $-40{ }^{\circ} \mathrm{C}$ was first exposed to but-3-en-1-ol then quenched with an excess of methanol, scheme 60. Indeed the desired bis-ether 71 was formed in a respectable $71 \%$ yield after purification by flash chromatography.

\footnotetext{
XIII Although this is a commonly used reagent for the intramolecular silylation of diols, at the time of these studies, the author was unaware of any reports of the intermolecular disilylation of alcohols.
} 




70

2) $\mathrm{MeOH}, 15 \min , 71 \%$

71

Scheme 60: Initial study on the dietherification of 70

To further confirm the viability of this tethering strategy, a second model study was conducted. A solution of 70 in DMF at $-40^{\circ} \mathrm{C}$ was exposed to one equivalent of but-3en-1-ol, followed by one equivalent of pyridine, then 1.1 equivalents of allyl alcohol, scheme 61. Pyridine was added to neutralise the liberated triflic acid. (As this method was to be adapted for the coupling of the B-hydroxy ketone to the mono substituted silyl tether, it was proposed that the liberated triflic acid could cause the acid catalysed dehydration of the B-hydroxy ketone). Again this modified strategy was successful with the desired bis-ether being 72 obtained in $65 \%$ after purification by flash chromatography.



Scheme 61: Second exploratory study into the dietherification of 70

Numerous attempts were then made at coupling the desired B-hydroxy ketone to the tertbutylsilyl tether, scheme 62. After the addition of one equivalent of but-3-en-1-ol, different ratios of pyridine, ranging from none to two equivalents were added before the addition of the B-hydroxy ketone. In some instances DMAP was added, either in catalytic or stoichiometric quantities, and the reaction temperature varied from $-40{ }^{\circ} \mathrm{C}$ to $80{ }^{\circ} \mathrm{C}$ 
after the initial addition of but-3-en-1-ol. However in the best scenario, with two equivalents of pyridine and catalytic DMAP, ${ }^{1} \mathrm{H}$ NMR analysis of the crude reaction mixture indicated that the desired bis-silylether $\mathbf{7 3}$ had been formed in only approximately $10 \%$ yield. Under most conditions, starting material and a small fraction of the unsaturated dienone $\mathbf{5 9}$ were isolated. Reversal of the addition of the alkoxy substrates, with the ß-hydroxy ketone being added before but-3-en-1-ol, once again resulted in the formation of the unsaturated compound $\mathbf{5 9}$ (refer to page 79).



Scheme 62: Attempted formation of the tert-butyl tethered ether

Furthermore, the attempted RCM of the previously synthesised 4-(3-butenoxy)-3(allyloxy)-di-tert-butylsilane 72, using either Grubbs' first or second-generation ruthenium catalyst, proved troublesome, scheme 63. Starting material was predominantly isolated, even after the addition of six mol \% of the second-generation catalyst $\mathbf{6 2}$. Thus, even in the eventuality of synthesising the desired B-keto substituted derivative $\mathbf{7 3}$, it seemed likely that this substrate would not be particularly amenable to RCM. Evidently, a di-tert-butyl silyl tether was not a viable option.

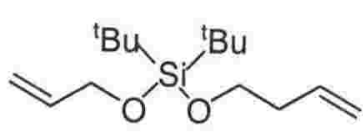

72



$\mathrm{CH}_{2} \mathrm{Cl}_{2}, \mathrm{rt}, 18 \mathrm{~h}$



74

Scheme 63: Attempted RCM of the dialkylated di-tert-butyl silyl derivative 72 


\section{2: Adaptation to a more linear approach for the synthesis of the C-12 to C-24 fragment of peloruside $A$}

In view of the previous results it appeared that a less than ideal linear synthesis, involving the incorporation of the silyl tether at a later stage in the synthesis, was required for the formation of pel $\mathrm{A}$, scheme 64 . This strategy commences with an aldol reaction between the suitably protected $\beta$-hydroxyketone 75 and pyranose $\mathbf{5 c} .{ }^{\mathrm{XIV}}$ The resulting hydroxyl substituent would then be benzylated, to facilitate a global debenzylation at a later stage of the synthesis, and the protecting group $\left(\mathrm{P}^{1}\right)$ removed to allow for attachment of the silyl tether. (S)-2-ethyl-but-3-en-1-ol is then added to the silyl tether, followed by RCM, to give key intermediate $\mathbf{7 6}$.



75



$5 c$



76



2) $\mathrm{RCM}$



1) Benzylation

2) Deprotection

3) $\mathrm{Cl}_{2} \mathrm{SiR}_{2}$



Scheme 64: A more linear approach for the synthesis of peloruside A.

\footnotetext{
${ }^{\mathrm{XIV}}$ It was anticipated that an asymmetric Mukiyama aldol reaction (using $(R)$ or $(S)$ BINOL as a ligand, or a boron mediated 1,5-anti aldol reaction (using PMB as a protecting group) would eventually be used to control the stereochemistry of this aldol reaction.
} 
The asymmetric reduction of $\mathbf{7 6}$ would be the challenging part in this synthesis. A series of reducing agents would need to be investigated to ascertain which choice of reagent would give the highest yield of the desired 1,3-anti product 77. If formation of the desired anti adduct proves elusive, it is anticipated that the ketone could be selectively reduced with $\mathrm{ZnBH}_{4}$ to give the syn product, which could be subsequently inverted by a Mitsunobu reaction. ${ }^{151}$ Similar chemical transformations to those previously discussed in the introduction, including the attachment of the C-1 to C-2 carbon framework, and a Yamaguchi macrolactonisation, are then required to complete the synthesis of pel A.



Scheme 65: Asymmetric reduction of key intermediate 77

In order to test the feasibility of this new synthetic strategy, and in particular the plausibility of the asymmetric reduction, model studies were conducted with pivaldehyde as a substrate for the pyranose ring. Thus, the B-hydroxyketones $\mathbf{2 4}$ were first protected, as either silyl derivatives, or the methoxymethylether (MOM) derivatives, schemes 66 and 67, respectively. Protection of the hydroxyl group as the methoxymethylether proceeded in noticeably lower yields than formation of the silyl derivatives.<smiles>C=C(P)C(O)CC(C)=O</smiles>

$24 a R^{2}=M e$

$24 b R^{2}=H$



$75 a R^{1}=T B S, R^{2}=M e, 73 \%$

$75 b R^{1}=$ TBS, $R^{2}=H, 74 \%$

$75 c R^{1}=$ TBDPS, $R^{2}=M e, 75 \%$

Scheme 66: Silylation of the B-hydroxy ketones 
<smiles>[R]C(=C)C(O)CC(C)=O</smiles>

$24 a R^{2}=M e$

$24 b R^{2}=H$



$75 \mathrm{~d} R^{2}=\mathrm{Me}, 32 \%$ *

75e $\mathrm{R}^{2}=\mathrm{H}, 48 \%$

*unoptimised

Scheme 67: Etherification of the B-hydroxy ketones

The subsequent aldol reactions of the protected B-hydroxy ketones with pivaldehyde as the aldehyde acceptor proceeded smoothly and provided some much needed reassurance that successful aldol reactions were indeed possible! Once again, yields for the reactions with the silyl protecting groups were higher than those with the MOM protecting group, suggesting that protection of the B-hydroxyketone as the methoxymethyl ether was not an optimum strategy, scheme 68. Consequently, further studies with the MOM derivatives were discontinued.<smiles>C=C(P)C(O)CC(C)=O</smiles>

$75 \mathrm{a} \mathrm{R}^{1}=\mathrm{TBS}, \mathrm{R}^{2}=\mathrm{Me}$

$75 b R^{1}=T B S, R^{2}=H$

$75 c R^{1}=$ TBDPS, $R^{2}=M e$

$75 d R^{1}=M O M, R^{2}=M e$
1) LTMP or LDA, THF, $-78{ }^{\circ} \mathrm{C}, 1 \mathrm{~h}$

2)<smiles>CC(C)(C)C=O</smiles>



$78 \mathbf{a} R^{1}=T B S, R^{2}=M e, 65 \%$ $78 b R^{1}=T B S, R^{2}=H, 68 \%$ $78 C R^{1}=$ TBDPS, $R^{2}=M e, 67 \%$

$78 d R^{1}=$ MOM, $R^{2}=M e, 56 \%$

Scheme 68: Model aldol studies with pivaldehyde

Benzylation of the aldol adducts however, proved to be more challenging than expected due to the presence of additional acidic protons $\alpha$ to the ketone. First aldol adduct $\mathbf{7 8 a}$ was exposed to one equivalent of $\mathrm{NaH}$ followed by benzyl bromide. However these conditions resulted in the decomposition of the starting material. In the event that excess $\mathrm{NaH}$ was causing the unwanted decomposition of the substrate, a $1 \mathrm{M}$ solution of 
potassium tert-butoxide in THF was used as the base as this can be more accurately measured than a $60 \%$ dispersion of $\mathrm{NaH}$ in mineral oil. However once again, the starting substrate decomposed. Obviously, a milder method of benzylating the hydroxyl substituent was required.

Attempts were then made at benzylating $78 \mathrm{a}$ by using a suspension of freshly prepared silver oxide and benzyl bromide in DMF. ${ }^{151}$ Yet again, these conditions resulted in the decomposition of the starting substrate. Analysis of the crude material by ${ }^{1} \mathrm{H}$ NMR indicated that the silyl group had been removed under these conditions. Reactions involving a change of solvent from DMF to $\mathrm{CH}_{2} \mathrm{Cl}_{2}$, or the addition of catalytic $\mathrm{KI}$ in an attempt to activate the alkyl halide, fared no better. In all instances, the substrate decomposed.

It was thus decided that the hydroxy group could be more satisfactory benzylated under mildly acidic conditions using benzyl trichloroacetimidate $\mathbf{7 9}$ as a benzyl donor. ${ }^{152}$ Benzyl trichloroacetimidate can be readily synthesised by the $\mathrm{NaH}$ catalysed addition of benzyl alcohol to trichloroacetonitrile. ${ }^{153}$ Fortunately, this protocol worked well with the benzyl adducts $80 \mathrm{~b}$ and $80 \mathrm{c}$ being synthesised in $72 \%$ and $71 \%$ respectively, scheme 69 .



$78 b R^{1}=T B S, R^{2}=H$ $78 \mathrm{C} \mathrm{R}^{1}=$ TBDPS, $\mathrm{R}^{2}=\mathrm{Me}$



$$
\begin{aligned}
& 80 b R^{1}=\text { TBS, } R^{2}=H, 72 \% \\
& 80 c R^{1}=\text { TBDPS, } R^{2}=M e, 71 \%
\end{aligned}
$$

Scheme 69: Benzylation of the aldol adducts with benzyl trichloroacetimidate 


\section{3: Revisiting the original cyclic bis-silyl ether strategy}

Although the above linear strategy was progressing at a satisfactory rate, ultimately this was not the most desirable approach. Since these aldol reactions could be successfully performed on the milligram scale and in respectable yields, it was apparent that there was nothing technically wrong with the manner in which the previous aldol reactions, involving the bis-silyl ethers, were being conducted. Some underlying phenomenon was preventing these cyclic bis-silyl ethers from successfully participating in aldol reactions.

After much consideration, it was proposed that a potential cause of our problems was the bis-silyl cyclic ethers containing sub-milligram quantities of ruthenium by-products. Indeed Maynard and Grubbs ${ }^{154}$ surmised in a recent article that the highly-coloured ruthenium complexes and the residual ruthenium that forms as a by-product during ruthenium alkylidene catalysed RCM reactions often cause problems such as olefin isomerization during product distillation and the decomposition of substrates over time. Thus it seemed only logical that the residual ruthenium was responsible for the problems encountered during the aforementioned aldol reactions.

To investigate this supposition, $\mathbf{6 5} \mathbf{b}$ was subjected to rigorous purification by preparative HPLC with detection by mass-spectromentry, before an aldol reaction between itself and pivaldehyde was conducted, scheme 70 . Gratifyingly, ${ }^{1} \mathrm{H}$ NMR analysis of the crude reaction mixture indicated that the desired aldol adduct $\mathbf{8 1 b}$ had been formed.



$65 b$
1) $L D A,-78^{\circ} \mathrm{C}, 90 \mathrm{~min}$

2)





81b

Scheme 70: Aldol reaction of $65 \mathrm{~b}$ purified by preparative HPLC 
Diisopropylsilyl ether 66d was then subjected to an aldol reaction with pivaldehyde, scheme 71. Again this aldol reaction was a success, with the aldol adduct $\mathbf{8 2}$ being produced in a respectable $59 \%$ yield. Diene $\mathbf{8 2}$ was then subjected to RCM with catalyst $\mathbf{6 2}$ to give the bis-silyl cyclic $\mathbf{8 1 d}$ in a $77 \%$ yield. Alternatively $\mathbf{6 5 d}$ was synthesised by the RCM of the acyclic adduct $66 \mathrm{~d}$ and the residual oil rigorously purified three times by flash chromatography, before being subjected to an aldol reaction with pivaldehyde, figure 71 . Fortunately, this aldol reaction was moderately successful with the desired aldol adduct $\mathbf{8 1 d}$ being formed in $30 \%$. Presumably this low yield was due to the purity of the starting material. However, more importantly, these studies indicated that aldol reactions of the cyclic bis-ethers are indeed viable, provided the cyclic ether is of adequate quality.



$66 d$

$6210 \mathrm{~mol} \%$ $\mathrm{CH}_{2} \mathrm{Cl}_{2}, \mathrm{rt}, 8 \mathrm{~h}$ $74 \%$ ( $x 3$ columns)



$65 d$
1) LTMP, $-78^{\circ} \mathrm{C}, 1 \mathrm{~h}$

2)



1) LTMP, $-78^{\circ} \mathrm{C}, 45 \mathrm{~min}$

2)





82

$6210 \mathrm{~mol} \%$ $\mathrm{CH}_{2} \mathrm{Cl}_{2}, \mathrm{rt}, 8 \mathrm{~h}$ $77 \%$



81d

Scheme 71: Racemic aldol studies 


\section{4: Removing the residual ruthenium}

Ideally, the problems encountered with the removal of the residual ruthenium could be largely alleviated if a more active, or polymer bound, RCM catalyst was used. Recently Hoyveda and Schrock reported the use of the chiral Mo-based catalysts, such as $\mathbf{8 3}$, to synthesise six and seven membered siloxanes bearing tertiary ether centres. ${ }^{155}$ Although such molybdenum catalysts are extremely air and

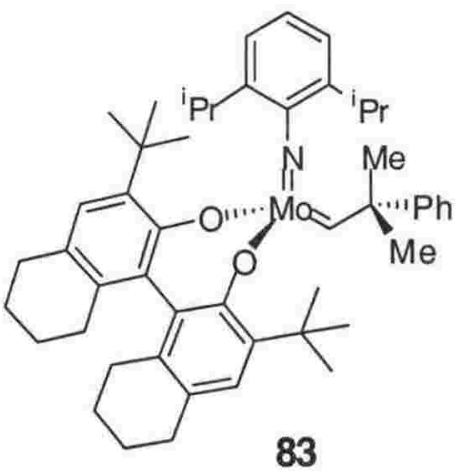
water sensitive, necessitating glove box conditions, the synthesis of the aforementioned siloxanes could be achieved using 1 to $5 \mathrm{~mol} \%$ of the molybdenum catalyst. Thus, it was anticipated that the RCM of our silyl-tethered ethers could also be achieved with low catalytic loadings (1-2 mol \%) of $\mathbf{8 3}$.

In preparation for the Mo-catalysed RCM, benzene was added to precursor $\mathbf{6 6} \mathbf{b}$ and the solvent removed under reduced pressure to allow for the azeotropic distillation of any residual water. This process was repeated three times. The attempted Mo-catalysed RCM of $\mathbf{6 6} \mathbf{b}$ was then conducted in a glove box, with the solution of the diene in benzene being stirred for $24 \mathrm{~h}$ at room temperature in a manner analogous to that reported in the literature, ${ }^{156}$ scheme 72 . Although this procedure was attempted on several occasions, with the catalytic loading being increased to $5 \mathrm{~mol} \%$, unfortunately only the acyclic ether was ever isolated. Obviously this was not a suitable catalyst for our substrate.

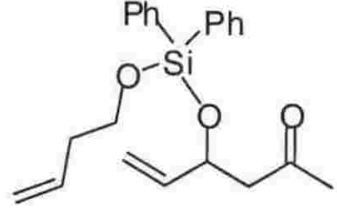

$66 \mathrm{~b}$

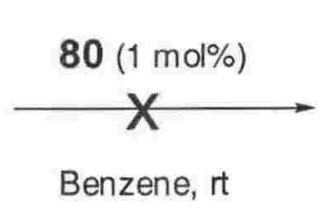

Benzene, it<smiles>CC(=O)CC1C=CCCO[Si](c2ccccc2)(c2ccccc2)O1</smiles>

$65 b$

Scheme 72: Attempted synthesis of $\mathbf{6 5} \mathrm{b}$ by Mo-catalysed RCM 
Even though more time could be invested in finding an alternative RCM catalyst, in the interim, a method for removing the residual ruthenium from the RCM of the bis-siloxy ethers using Grubbs' second-generation catalyst was required. Purification by preparative HPLC was too onerous to be synthetically useful. Reports of the purification of RCM adducts by the addition of water soluble tris(hydroxymethyl)phosphine, as a ligand to capture any unwanted ruthenium, indicated that this might a viable option. ${ }^{155}$ However, although readily available, $\mathrm{P}\left(\mathrm{CH}_{2} \mathrm{OH}\right)_{3}$ is still a somewhat expensive reagent. Other procedures for the removal of ruthenium by-products formed from the Grubbs reagent include the use of the somewhat toxic $\mathrm{Pb}(\mathrm{OAc})_{4}$ to oxidise the ruthenium metal, ${ }^{156}$ or treatment with $\mathrm{Ph}_{3} \mathrm{PO}$ or DMSO. ${ }^{157}$ These methods allowed for the reduction of the ruthenium levels down to approximately $1-2 \mu \mathrm{g}$ per $5 \mathrm{mg}$ of product(s).

More recently though, a superior methodology for the removal of residual ruthenium from RCM reaction products was reported. This involves the sequential treatment of the RCM adduct with silica gel, activated carbon (50 weight equivalent relative to the crude products), and column chromatography on silica gel, to give compounds with residual ruthenium levels of $0.06-0.53 \mu \mathrm{g}$ per $5 \mathrm{mg}$ of product. ${ }^{158}$ Requiring both inexpensive and non-toxic reagents, this methodology was successively applied for the purification of our RCM adducts. Thus, the yields previously reported in Table 2 are those after purification by this methodology.

A future objective of this research however, is to find a more suitable RCM catalyst for this transformation. Either a more active RCM catalyst that can be used in lower catalytic loadings, or a polymer bound catalyst that can be recycled and hence removed from the reaction mixture by simple filtration, is required. Two recent advancements in this area, both by Blechert et. al., include the highly active ruthenium metathesis catalyst $84^{159}$ or the polymer bound derivative $\mathbf{8 5},{ }^{160}$ figure 19 . It is anticipated that the applicability of these, or similar alternatives, will be explored at a later date. 




84



85

Figure 19: Alternative RCM catalysts

Nevertheless, for the time being, a suitable strategy for the racemic synthesis, and subsequent aldol reaction, of the C-12 to C-24 fragment of pel A had been developed. It was now time to extend these basic principles and apply them to the asymmetric synthesis of this fragment. 


\section{Chapter 6}

\section{Asymmetric synthesis of the C-12 to C-24 fragment of pelourside $\mathrm{A}$}

\section{1: Key stereochemical features}

With guidelines in place for the synthesis of the C-12 to C-24 fragment of pel A, the next challenge was to synthesise this fragment asymmetrically and develop a methodology for its stereoselective coupling to the pyranose ring. Due to the apparent structural similarity between pel A and the 16 membered macrolide, epothilone B, figure 20 , it was initially thought that pel A may act as an epothilone surrogate by binding to a common site on B-tubulin. ${ }^{\mathrm{Xv}}$ Initially our focus was on the synthesis of the enantiomer shown below, however a flexible strategy was planned which would allow for the synthesis of either enantiomer of pel A.

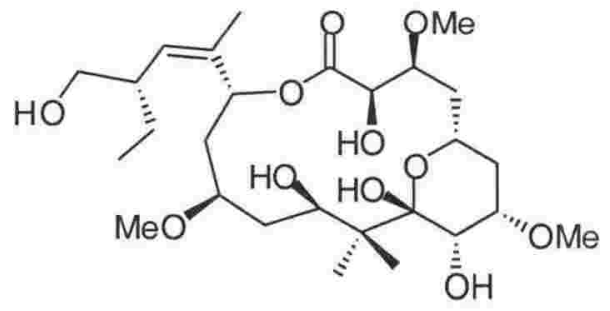

peloruside $A$



epothilone B

Figure 20: Structural similarities between peloruside A and epothilone B

\footnotetext{
${ }^{\mathrm{Xv}}$ This appeared to be a common misconception by many in the academic community. Until its absolute stereochemistry was determined, all publications concerning the synthesis of pel A focussed on synthesising the enantiomer shown.
} 
A review of the retrosynthetic analysis for pel A highlights the required 1,5-anti relationship between the C-11 and C-15 stereocentres, scheme 73 . To achieve this 1,5anti relationship, a boron-mediated aldol reaction between the C-12 to C-24 fragment of pel $\mathrm{A}$ and the aldehyde acceptor of the pyranose ring will be performed. Although Evans $^{161}$ and Paterson ${ }^{162}$ pioneered this area of remote 1,5-induction in boron-mediated aldol reactions, to-date only limited explanations have been given for this surprising phenomenon. Thus, in achieving the 1,5-anti relationship required for the synthesis of pel $\mathrm{A}$, it is anticipated that further insight into the origin of these boron-mediated reactions will be gained.


Scheme 73: Retrosynthesis of peloruside A showing the required 1,5-anti relationship between the C-11 and C-15 stereocentres with the C-12 to C-24 framework in red

A review of the synthesis of the C-12 to C-24 fragment of pel $\mathrm{A}$ is given in scheme 74. The cyclic bis-silyl ether $\mathbf{2 2}$ is the tethered version of the side chain shown in red above. However, for simplicity, the ethyl side chain will be once again omitted. The focus of this chapter is thus on asymmetrically synthesising the B-hydroxyketone $\mathbf{2 4}$ and developing an appropriate protocol for the boron-mediated 1,5-anti aldol reaction of the bis-silyl ether. These two concepts will be discussed in turn.



Scheme 74: Retrosynthesis for the formation of the C-12 to C-24 fragment of pel A 


\subsection{Synthesis of $(R)$-4-hydroxy-5-methylhex-5-en-2-one}

From the outset, we were aware of a published procedure for the synthesis of the desired $(R)$-4-hydroxy-5-methylhex-5-en-2-one 24 . This required a diisopinocamphylborane mediated enantioselective aldol reaction between acetone and methacrolein. ${ }^{163}$ However, as the enantiomeric excess for this reaction was $73 \%$ with a yield of $68 \%$, we optimistically proposed that an alternative, and indeed better, synthesis of this target compound could be developed. Upon reviewing the literature, two general strategies for the enantioselective synthesis of this ß-hydroxy ketone emerged: a) the kinetic resolution of the racemic alcohol and b) an alternative enantioselective aldol reaction.

\subsection{1: The kinetic resolution of $( \pm)-4$-hydroxy-5-methylhex-5-en-2-one}

The kinetic resolution of racemic substrates can be performed using one of two approaches, figure $21 .^{164}$ The first, and more established approach is a classic kinetic resolution. Here resolution of the enantiomers $(R+S)$ occurs when $k_{R} \neq k_{S}$ and when the reaction is stopped at some stage at or near $50 \%$ conversion. The enantiomers are then separated by obtaining the product $(\mathrm{P})$, formed from $\mathrm{R}$, and the non-converted starting substrate $(\mathrm{S})$. A difference in the specific rate constants $\left(\mathrm{k}_{\mathrm{R}}, \mathrm{k}_{\mathrm{S}}\right)$ is obtained by using a chiral catalyst, which may be either chemical or biological nature. Dynamic resolution however, encompasses both classic resolution and an additional racemising process, $\mathrm{k}_{\mathrm{rac}}$. Again substrate racemisation may be achieved either by chemical or biological catalysis and, provided that $\mathrm{k}_{\mathrm{rac}}>\mathrm{k}_{\mathrm{S}}$, all of the substrate is transformed into a single product enantiomer in $100 \%$ theoretical yield. Due to the enhanced yields of dynamic kinetic resolution, this is clearly the more desirable strategy for the resolution of racemic substrates. 


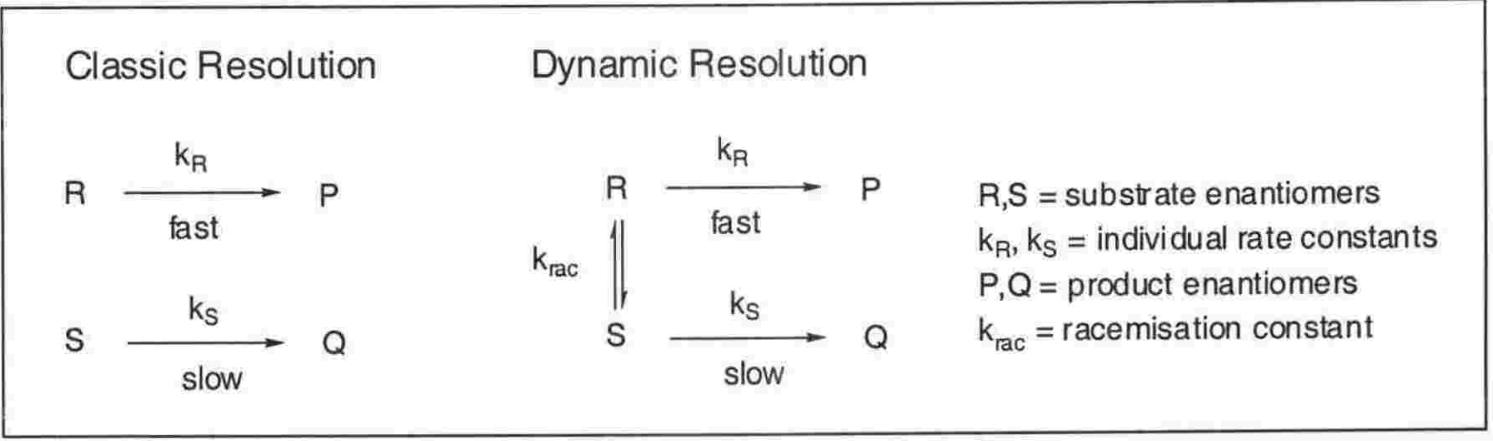

Figure 21: Classic versus Dynamic Kinetic Resolution

However, upon reviewing the procedures required for dynamic kinetic resolutions, it appeared that our B-hydroxy ketone would not be particularly amenable to such methodology. Typically the dynamic kinetic resolution (DKR) of secondary alcohols involves the transition metal-catalysed racemisation of the alcohol coupled with enzymatic resolution. ${ }^{165}$ Ruthenium is frequently the transition metal of choice, with the racemisation of the substrate commencing with a base-mediated hydrogen abstraction from a hydrogen donor to form the intermediate ruthenium alkoxy species $\mathbf{I}$, scheme 75. ${ }^{166 \mathrm{a}}$ Abstraction of the $\alpha$-proton then gives the intermediate ketone and a ruthenium hydride complex II. Finally, the subsequent readdition of the hydrogens to the ketone completes the catalytic cycle to give the racemised substrate.



I

II

Scheme 75: Mechanism of the ruthenium-catalysed hydrogen transfer reaction

Due to the presence of a ketone moiety in our starting substrate, it is unlikely that such a DKR is feasible for the following reasons, scheme 76. First, the $\alpha$-ketone proton could be 
preferentially abstracted leading to the $\alpha, \beta$-unsaturated adduct $\mathbf{A}$, second, the original ketone moiety itself could be reduced to give $\mathbf{C}$, and third, readdition of the hydrogens in a 1,4-fashion would give the diketone D. A biocatalytic DKR of our B-hydroxy ketone, where dehydrogenases are required for the oxidative process and redox-enzymes are required for the reductive process, ${ }^{165}$ could also suffer a similar fate.



Scheme 76: Proposed products from the in situ racemisation of the starting substrate during the attempted DKR of the B-hydroxy ketone

Thus, in light of the potential pitfalls of the DKR of the B-hydroxy ketone, investigations into classical kinetic resolution techniques were made. Traditionally this requires the use of an esterase to resolve the secondary alcohol, however more recently, non-enzymatic strategies ${ }^{166}$ such as palladium catalysed sparteine oxidative kinetic resolutions, ${ }^{167}$ the Sharpless resolution of allylic alcohols, ${ }^{168}$ or Fu's azaferrocene catalysed enatioselective acylation, ${ }^{169}$ have been developed.

Unfortunately, upon closer examination of the non-enzymatic strategies, some potential problems were highlighted. For example, the palladium catalysed oxidative kinetic resolution requires the use of the naturally occurring chiral base, (-)-sparteine, which unfortunately can only be obtained in one enantiomeric form. Unlike traditional esterase resolutions, where either enantiomer can be obtained either directly, or by the deacetylation of acetylated alcohol, if the undesired enantiomer is formed during this 
oxidative strategy, there are no easy methods of obtaining the correct enantiomer. The strategy by $\mathrm{Fu}$ also presented some practical difficulties as the synthesis of the azaferrocene catalyst required the use of preparative chiral HPLC, which was not readily available. Although the Sharpless methodology appeared to offer some promise as either enantiomer can be synthesised, due the extremely stringent conditions required for effective epoxidation strategies and the potentially explosive nature of the requisite tertbutyl hydroperoxide solution, we initially opted to explore alternative strategies.

Thus, the more traditional approach of resolving secondary alcohols using esterases was investigated. Enantioselective esterases are particularly useful enzymes for the kinetic resolution of secondary alcohols due to their efficiency, their applicability to a wide range of substrates and the ease at which the reactions can be performed on a large scale. Unfortunately though, there is little X-ray structural information available about these esterases and the appropriate enzyme is usually chosen by screening. Hence two candidates, lipases from Candia antartica ${ }^{170}$ and Pseudomonus cepia ${ }^{171}$ were investigated for their ability to enantioselectively esterify, and hence kinetically resolve our ß-hydroxy ketone.

The racemic B-hydroxyketone $\mathbf{2 4 b}$ was dissolved in tert-butylmethyl ether with the appropriate esterase and isopropenyl acetate as the acyl donor, scheme 77 . The heterogenous slurry was then stirred rapidly at room temperature with progress of these reactions being monitored by ${ }^{1} \mathrm{H}$ NMR. After seven days for the Candida antartica lipase, and five days for the Pseudomonus cepia lipase, the reactions reached 50\% completion and the lipase was removed by filtration. To determine the enantiomeric excess of these esterification reactions, the $\beta$-hydroxy ketone was separated from the acetylated compound by flash chromatography and a portion converted into the TBS silyl ether. This silylation was necessary in order to make a less polar and bulkier derivative that was more amenable to analysis by chiral gas chromatography. Unfortunately though, chiral gas chromatography revealed that the enantiomeric excess for both esterases was extremely low at approximately $5 \%$. 


<smiles>C=C(C)[C@H](CC(C)=O)OC(C)=O</smiles>

86

Scheme 77: Attempted kinetic resolution of ( \pm )-4-hydroxy-5-methylhex-5-en-2-one using lipase from either Candida antartica or Pseudomonus Cepia

In many respects these results were not surprising. According to the empirical rules of Kazlauskas, ${ }^{172}$ lipase stereoselectivity is set mainly by steric interactions between the enzyme and substrate, with the enantioselectivity of the esterification of secondary alcohols depending upon the relative size of the two neighbouring groups. Thus, if the groups are similar, as with our B-hydroxyketone, poor resolution is obtained. Although non-steric interactions between the enzyme and the substrate can provide for enantioselective differentiation, the electron density of both the carbonyl and olefinic functional groups of the $\beta$-hydroxy ketone provided for little electronic differentiation.


particularly viable and investigations into alternative enantioselective aldol reactions were conducted.

\subsection{2: Asymmetric aldol reaction}

The search for catalysts that mimic the selectivity of biological aldolases has been the subject of intense efforts in recent years. Many catalysts, including the $(R)$ $\mathrm{LnLi}_{3}$ tris(binapthoxide) catalyst 87 developed by Shibasaki et. al. ${ }^{173}$ and the dinuclear $\mathrm{Zn}$ catalyst $\mathbf{8 8}$ developed by Trost et. al. ${ }^{174}$ offered potential for the synthesis of our desired B-hydroxy ketone, however the recently developed procedure by List for the $(L)$-proline 
(89) catalysed asymmetric aldol reaction between acetone and a variety of aldehydes, ${ }^{175}$ appeared to be the more straightforward strategy. Although the reaction between methacrolein and acetone was not reported, we proposed that List's methodology could be adapted for the synthesis $(R)$-4-hydroxy-5-methyl-5-hexen-2-one.



Thus, $30 \mathrm{~mol} \%$ of $(L)$-proline was added to a solution of methacrolein in $4: 1 \mathrm{v} / \mathrm{v}$ DMSO:acetone in a procedure analogous to that reported by List, scheme 78 . Initially we had concerns that the $\alpha, \beta$-unsaturated ketone would be preferentially formed, via a proline catalysed elimination reaction, but with relief, the desired ß-hydroxyketone $\mathbf{2 4}$ was formed in a modest $38 \%$ yield. The replacement of DMSO by acetone, which has been reported to improve the yields of some proline catalysed reactions, ${ }^{176 \mathrm{~b}}$ was unfruitful in our particular studies.



Scheme 78: $(L)$-proline catalysed asymmetric aldol reaction for the synthesis of $(R)-4$ hydroxy-5-methylhex-5-en-2-one 
At present it is assumed that this asymmetric aldol reaction occurs via an enamine mechanism with proline acting as a "micro-aldolase" that provides both the nucleophilic amino group and an acid/base co-catalyst in the form of the carboxylate, scheme $79 .{ }^{176 a}$ The enantioselectivity can be explained with a metal-free version of a ZimmermanTraxler type transition state, ${ }^{83}$ with the tricyclic hydrogen bonded framework accounting for the enantiofacial selectivity. To determine the enantiomeric excess of this reaction, the B-hydroxy ketone was again protected as the tert-butyldimethylsilyl ether. The GC trace of the silylated reaction product is shown in figure 22 overleaf, with the $(R)$ enantiomer, being formed in a respectable $74 \%$ ee. $^{\mathrm{xVI}}$

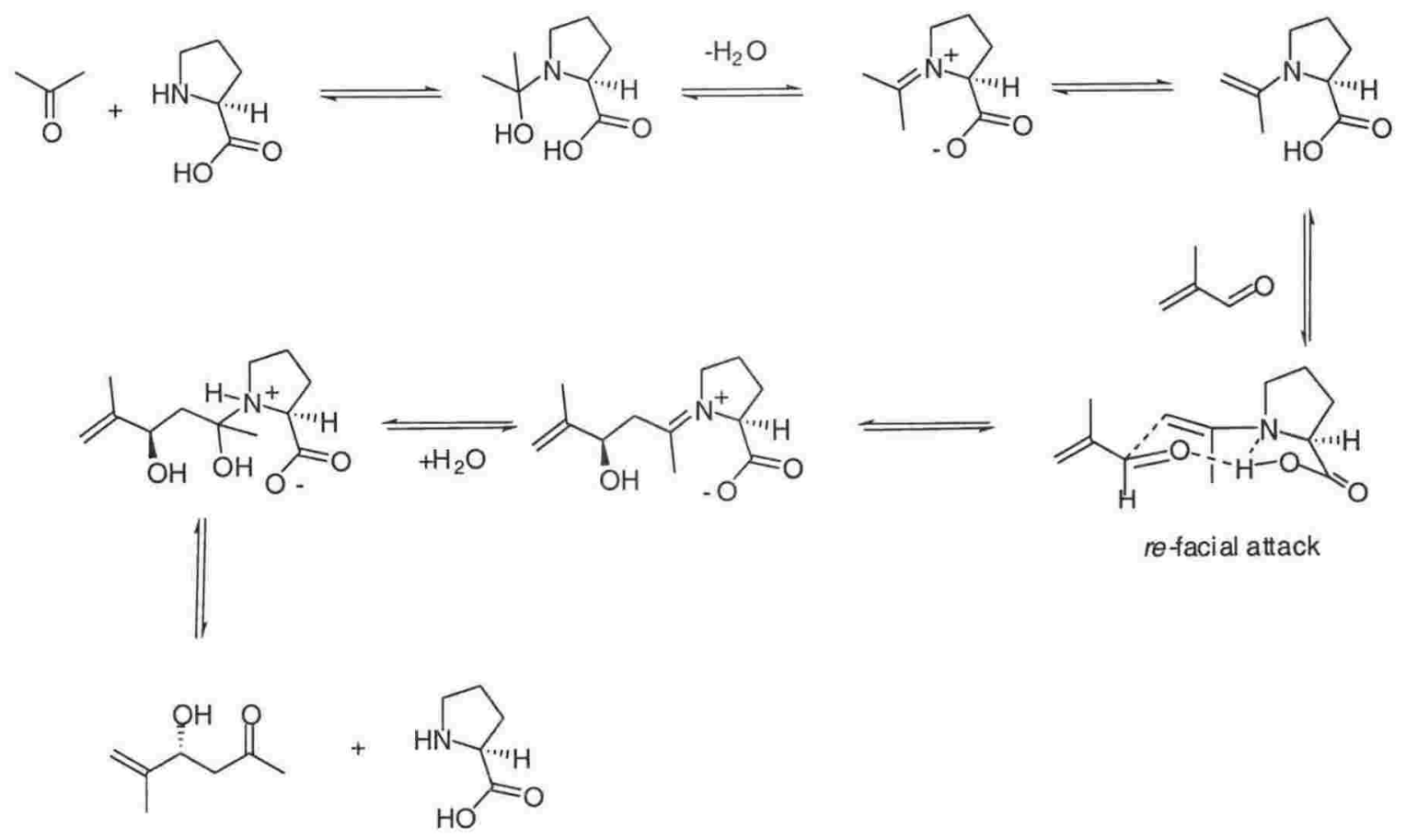

Scheme 79: Proposed mechanism the $(L)$-proline catalysed aldol reaction

${ }^{\mathrm{x} I}$ Precedent from List's work enables use to assign this as the major enantiomer 


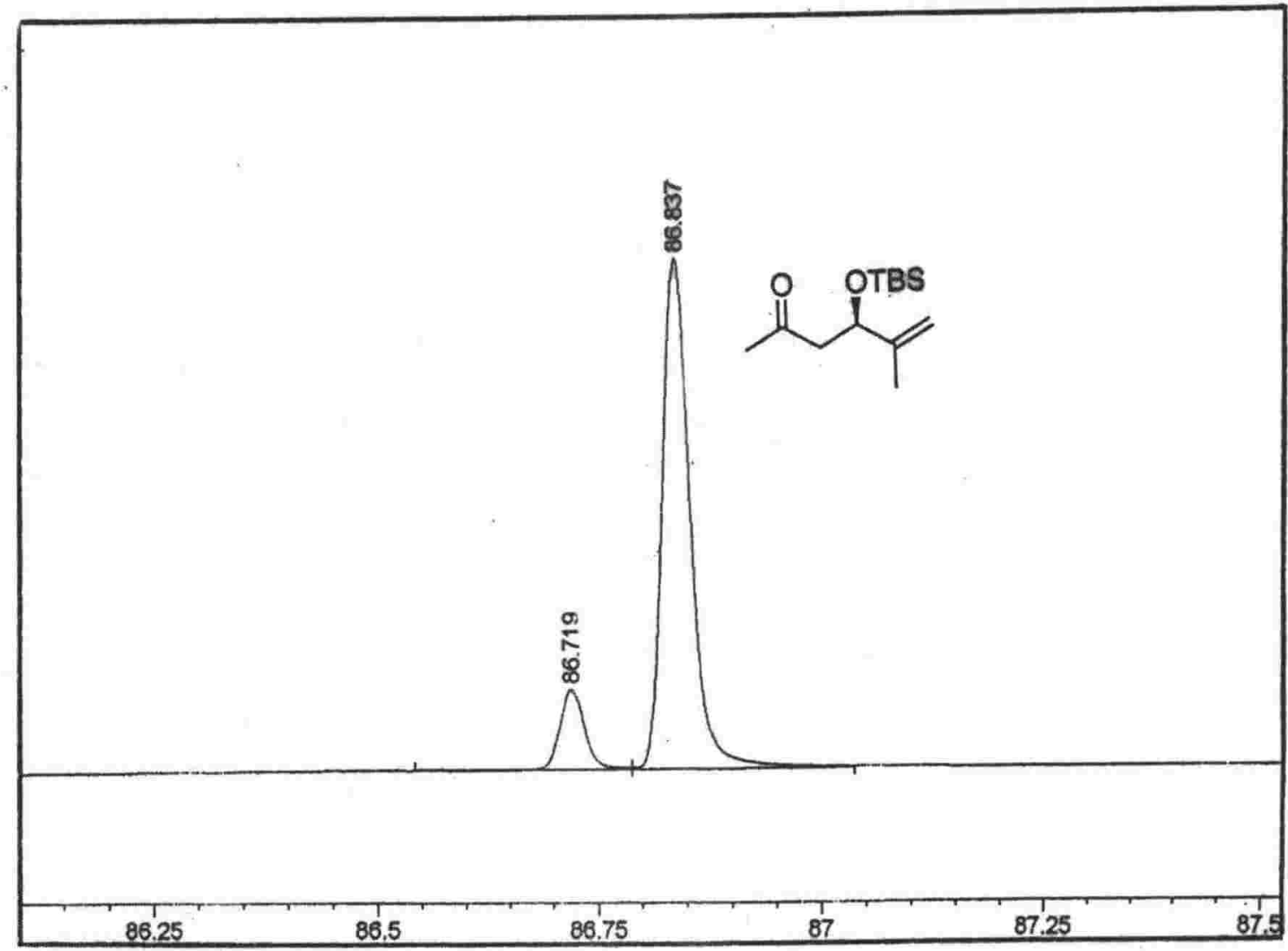

Figure 21: GC trace of the silylated enantiomers.

Unfortunately, when this methodology was repeated with acrolein as the aldehyde substrate, none of the desired aldol product was observed. This result, was however, in accordance with observations made by List, whereby significant proportions of $\alpha, \beta-$ unsaturated ketones were formed during the proline catalysed aldol reactions of $\alpha$ unsubstituted aldehydes. ${ }^{176 \mathrm{~b}}$ It is believed that this unsaturation occurs as a result of a Mannich-type condensation reaction, and not by the proline catalysed dehydration of the B-hydroxy ketone.

Nevertheless in the interim, a suitable strategy for the synthesis of $(R)$-4-hydroxy5-methylhex-5-en-2-one had been developed. Although the yields for this proline catalysed asymmetric aldol reaction are modest, due to the inexpensive, non-toxic and 
straightforward nature of this reaction, it was concluded that, for the synthesis of the $(R)$ 4-hydroxy-5-methyl-hex-5-en-2-one, this was the best strategy. ${ }^{x V I I}$ Thus, our next major challenge was to develop methodology suitable for obtaining the desired 1,5-anti aldol product. However before our proposed methodology for achieving this transformation is discussed, it is instructive to first highlight what is already known about boron mediated 1,5-anti aldol reactions.

\subsection{Boron mediated 1,5 -anti aldol reactions}

\subsection{1: A Brief Overview}

The boron mediated 1,5-anti aldol reaction commences with the enolisation of a ketone, using a base (either triethylamine or diispropylethylamine) and the appropriate boron reagent, $\mathrm{L}_{2} \mathrm{BX}$, (where $\mathrm{L}=$ alkyl ligand and $\mathrm{X}=\mathrm{Cl}$ or OTf). The enol boronate then nucleophilically attacks an aldehyde moiety, forming a boryl ether. Oxidative workup ( $\mathrm{MeOH}, \mathrm{H}_{2} \mathrm{O}_{2}$, pH 7 buffer solution) liberates the B-hydroxyketone. The notable feature of these reactions, such as the one illustrated in scheme 80 , is the often remarkable diastereoselectivity observed in the product B-hydroxy ketone. More often than not, the 1,5 -anti adduct is favoured. ${ }^{176}$

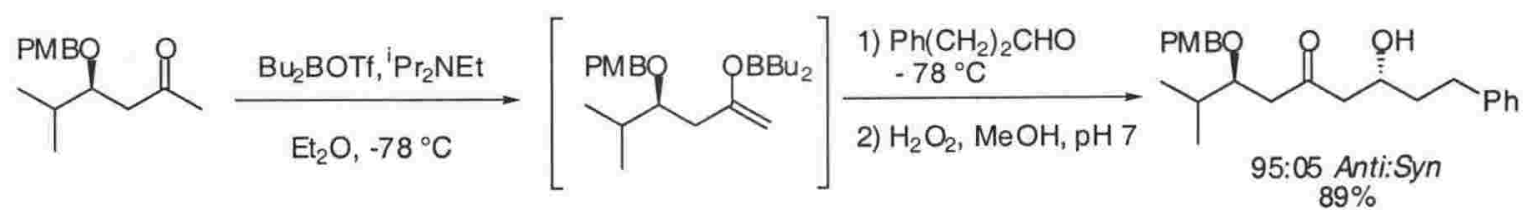

Scheme 80: The basic boron mediated 1,5-anti aldol reaction

\footnotetext{
${ }^{\mathrm{XVII}}$ Although (D) Proline is commercially available, it is more expensive than its (L) counterpart and hence an alternative procedure for the synthesis of the $(S)$ - $\beta$-hydroxy ketone will be sought. In the future, investigations will be made into the BINOL-Ti catalysed Mukaiyama aldol reactions between the silyl enol ether of acetone and methacrolein. Refer to reference 93.
} 
Although the diastereoselectivities of these reactions are enhanced by a variety of factors, including the choice of boron reagent and the local asymmetric environment, the primary influence in these reactions has long been speculated to be an electrostatic effect exerted by the B-alkoxy substituent. ${ }^{162 a, 177}$ Consequently, the nature of the B-oxygen protecting group is critical in determining the level of asymmetric induction. ${ }^{163}$ In general, high $1,5-$ anti selectivities are almost always obtained when the B-hydroxy protecting group is a para-methoxybenzyl ether or a benzylidine acetal ${ }^{162 a, 163}$ and as a result these protecting groups are used almost exclusively, figure 23. To date, no studies or models have been reported for this surprising process. However, the preference for aromatic substituents at the $\beta$ position initially suggested to us that a favourable $\pi$-stacking interactions may, at least, be partially responsible for the observed stereochemical outcome.<smiles>CC(=O)C[C@H]1CCO[C@H](c2ccccc2)O1</smiles>



1) $\mathrm{Bu}_{2} \mathrm{BOTf}$ 2) $\mathrm{Ph}\left(\mathrm{CH}_{2}\right)_{2} \mathrm{CHO}$<smiles>O=C(C[C@@H](O)CCc1ccccc1)C[C@@H]1CCO[C@H](c2ccccc2)O1</smiles>
>93:07 Anti:Syn $52 \%$



Figure 23: Examples of boron mediated 1,5-anti aldol reactions

The phrase ' $\pi$-stacking' signifies, in its simplest form, the positioning of a multiple bond (typically either C-O or C-C) parallel to and at approximately $3.5 \AA$ over an aromatic ring. ${ }^{178}$ Compelling evidence suggests that an attractive interaction is involved in these $\pi-\pi$ interactions, yet the exact nature of the $\pi$-stacking effect (either charge transfer or simple Van der Waals forces) is still a subject of some debate in the synthetic and computational arenas. ${ }^{179}$ Nevertheless, since the first synthetic application of $\pi$ stacking interactions by Corey et. al. in 1972 for the synthesis of the 
prostaglandins ${ }^{179}$ designed $\pi$-stacking effects have been encouraged for the control of asymmetric bond forming reactions, under both stoichiometric (chiral auxiliaries) and catalytic conditions, and more recently, for the purpose of assisting in molecular recognition. ${ }^{179}$

Some examples where $\pi$ stacking effects have been applied in a stoichiometric manner are given in figure 24 . These include the use of (-)-8-phenylmenthol derivatives in both asymmetric [3+2] cycloadditions catalysed by $\mathrm{Ni}(\mathrm{COD})_{2}$, equation (1), ${ }^{180}$ and in nucleophilic additions with Grignard reagents, equation (2). ${ }^{181}$ Perhaps the most focussed study to date in this area however, was reported by Evans et. al. in $1987 .{ }^{182}$ By changing the substituents on a oxazolidinone dieneophile, it was unambiguously established that appropriately orientated aromatic rings, such as that shown in equation (3), contribute to the enhanced diastereoselectivity of the Diels-Alder reactions of $\alpha, \beta$-unsaturated $N$ acyloxazolidinones.


Figure 24: $\pi$ stacking effects in asymmetric synthesis. 


\subsection{2: The proposed transition state for the boron mediated 1,5-anti induction in the synthesis of peloruside $A$}

Coupling the C-12 to C-24 fragment of pel A to the pyranose requires efficient anti-induction between $\mathrm{C}-11$ and $\mathrm{C}-15$ in which the stereochemical controlling element is the silyl protected $\beta$-hydroxy substituent, scheme 81 . Consequently, it was postulated that the proximity of the initially formed boron enolate to the phenyl substituents on the silyl tether, where $\mathrm{R}^{1}=\mathrm{Ph}$, would result in favourable $\pi$-stacking interactions and hence a transition state geometry leading to efficient 1,5-anti induction.<smiles>[R7]C1=C[C@@H](CC)CO[SiH]([R])O[C@@H]1CC(C)=O</smiles>

$\mathrm{L}_{2} \mathrm{BX}$
1) $L_{2} B X$

2)<smiles>COC(=O)C(O)C(O)C[C@H]1C[C@@H](OC)[C@H](O)[C@](O)(C(C)(C)C=O)[C@H]1O</smiles>

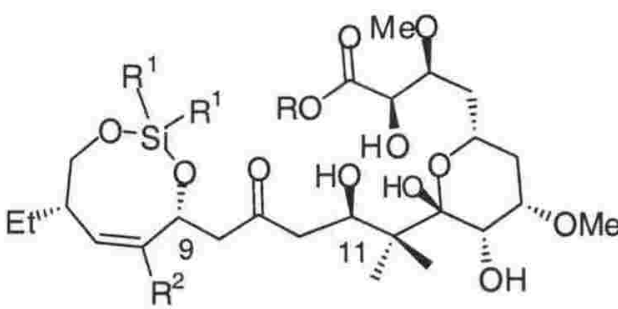

$5 d$


Scheme 81: Proposed transition state for asymmetric control

The enolate would adopt the geometry, as depicted in scheme 81 , to minimise the steric interactions between the cyclohexyl boron substituents and the phenyl substituents. The aldehyde $\mathbf{5} \mathbf{d}$ thus approaches the enolate from the opposite face to the phenyl substituent 
and chelates to the boron enolate though a twist boat transition state with the large alkyl group on the aldehyde adopting a geometry away from the phenyl ring and the aldehyde proton orientated towards the phenyl ring. Although one normally expects chelation to occur via a chair transition state, molecular calculations indicate that the twist-boat transition state for analogous boron mediated aldol reaction is energetically more favourable, by 1 to $4 \mathrm{kcal} / \mathrm{mol} .{ }^{183}$ In sum, this sequence of events leads to the desired 1,5 anti product.

\subsection{3: Studies into the synthesis of peloruside $A$}

To investigate this hypothesis, both the acyclic 66 and cyclic 65 ketone derivatives were subjected to boron mediated aldol reactions, with pivaldehyde being used as a model for the pyranose ring, scheme 82 . The enantiomerically pure derivatives, where $\mathrm{R}^{2}=\mathrm{Me}$, were synthesised from $(R)$-4-hydroxy-5-methylhex-5-en-2-one using the procedures detailed in chapter 5. By comparing both the acyclic and cyclic derivatives, insight into the supposed 'electrostatic effect' exerted by the B-alkoxy substituent could be gained. Similarly, being comparable in size, the effects of both the isopropyl and phenyl silyl tethers $\left(\mathrm{R}^{1}\right)$ were investigated as this enabled distinctions to be made between the electrostatic and steric effects of these tethers. The effects of the boron ligands, where $\mathrm{L}=$ cyclohexyl or (-)-diisopinocampheyl were also investigated. Generally these boron mediated reactions proceeded smoothly and in good yields. The diastereoselectivity of these reactions was determined by ${ }^{13} \mathrm{C}$ NMR analysis of the crude substrate, vide infra, and results of these studies are summarised in Table 4 overleaf. 
<smiles>[R7]C(=C)[C@H](CC(C)=O)O[Si]([R])([R])OCCC=C</smiles>

66
1) (-)- $-\mathrm{pcCl}, \mathrm{Et}_{2} \mathrm{O}$

$-78^{\circ} \mathrm{C}, 30 \mathrm{~min}$

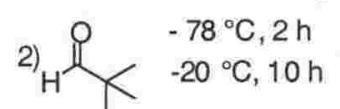

3) $\mathrm{H}_{2} \mathrm{O}_{2}, \mathrm{MeOH}, \mathrm{ph} 7$

1) $(-)-\mathrm{lpcCl}, \mathrm{Et}_{2} \mathrm{O}$

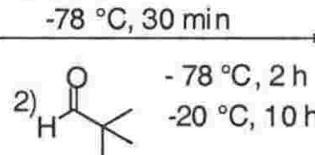

3) $\mathrm{H}_{2} \mathrm{O}_{2}, \mathrm{MeOH}, \mathrm{ph} 7$<smiles>[R7]C(=C)[C@H](CC(=O)C[C@@H](O)C(C)(C)C)O[SiH]([R])OCCC=C</smiles>

90<smiles>[R7]C(=C)[C@H](CC(=O)C[C@@H](O)C(C)(C)C)O[Si]([R])([R])OCCC=C</smiles>

91<smiles>[R]C1=CCCO[SiH]([R])C1CC(C)=O</smiles>

65

Scheme 82: Boron mediated 1,5-anti-aldol studies

Table 4: Boron mediated 1,5-anti aldol studies

\begin{tabular}{llllllll}
\hline Entry & Substrate & $\mathbf{R}^{1}$ & $\mathbf{R}^{\mathbf{2}}$ & $\begin{array}{l}\text { Boron } \\
\text { Reagent }\end{array}$ & \multicolumn{2}{c}{ Yield } & \multicolumn{2}{c}{ Diastereoselectivity } \\
\hline 1 & $\mathbf{6 6 a}$ & $i \mathrm{Pr}$ & $\mathrm{Me}$ & $(-)-\mathrm{Ipc}_{2} \mathrm{BCl}$ & $93 \%$ & $2.2: 1$ & $(\mathbf{9 0 a}: 91 \mathbf{a})$ \\
2 & $\mathbf{6 6 a}$ & $i \mathrm{Pr}$ & $\mathrm{Me}$ & $\mathrm{Cy}_{2} \mathrm{BOTf}$ & $97 \%$ & $1.2: 1$ & $(\mathbf{9 0 a}: 91 \mathbf{a})$ \\
$3^{\mathrm{a}}$ & $\mathbf{6 6 b}$ & $\mathrm{Ph}$ & $\mathrm{H}$ & $(-)-\mathrm{Ipc}_{2} \mathrm{BCl}$ & $98 \%$ & $2: 1$ & $\mathbf{( 9 0 b : 9 1 b )}$ \\
$4^{\mathrm{a}}$ & $\mathbf{6 5 a}$ & $i \mathrm{Pr}$ & $\mathrm{H}$ & $\mathrm{Cy}_{2} \mathrm{BOTf}$ & $85 \%$ & $1.2: 1$ & $\mathbf{( 9 2 a : 9 3 a )}$ \\
5 & $\mathbf{6 5 b}$ & $i \mathrm{Pr}$ & $\mathrm{Me}$ & $(-)-\mathrm{Ipc}_{2} \mathrm{BCl}$ & $88 \%$ & $1.5: 1$ & $\mathbf{( 9 2 b : 9 3 b )}$ \\
6 & $\mathbf{6 5 b}$ & $i \mathrm{Pr}$ & $\mathrm{Me}$ & $\mathrm{Cy}_{2} \mathrm{BOTf}$ & $95 \%$ & $1.2: 1$ & $\mathbf{( 9 2 b : 9 3 b )}$ \\
7 & $\mathbf{6 5 c}$ & $\mathrm{Ph}$ & $\mathrm{Me}$ & $(-)-\mathrm{Ipc}_{2} \mathrm{BCl}$ & $94 \%$ & $>99: 1$ & $\mathbf{( 9 2 c : 9 3 c )}$ \\
8 & $\mathbf{6 5 c}$ & $\mathrm{Ph}$ & $\mathrm{Me}$ & $\mathrm{Cy}_{2} \mathrm{BOTf}$ & $68 \%{ }^{\mathrm{b}}$ & $>99: 1$ & $\mathbf{( 9 2 c : 9 3 c )}$ \\
$9^{\mathrm{a}}$ & $\mathbf{6 5 d}$ & $\mathrm{Ph}$ & $\mathrm{H}$ & $(-)-\mathrm{Ipc}_{2} \mathrm{BCl}$ & $60 \%{ }^{\mathrm{b}}$ & $>99: 1$ & $\mathbf{( 9 2 d : 9 3 d )}$ \\
\hline
\end{tabular}

performed using the racemic substrate; ${ }^{\mathrm{b}}$ unoptimised.

The most striking observation from the above studies is the poor diastereoselectivities observed for all the acyclic derivatives, entries 1,2 and 3. Although moderate improvements in the diastereoselectivities are observed with the use of $(-)-\mathrm{Ipc}_{2} \mathrm{BCl}$ (compare entries 1 and 2), the differences in the ratio of anti to syn products are 
negligible. (-)-Ipc $2 \mathrm{BCl}$ is reported to improve the $\pi$-facial selectivity for the antiadducts. ${ }^{184}$ Thus, from this result alone, one can conclude that it is not the electrostatic effect of the $\beta$-siloxy substituent that is predominantly influencing $\pi$-facial discrimination in these boron mediated aldol reactions.

When comparing the aldol reactions of the cyclic derivatives (entries 4 to 9 ) some striking observations are made. With the isopropyl silyl tether (entries 4, 5 and 6), once again poor diastereoselectivities are observed. This is particularly evident in the ${ }^{13} \mathrm{C} N \mathrm{NR}$ spectra with the two diastereomers producing different chemical shifts as expected. For example the spectrum of 7,11-O-diisopropylsilanediyl-2,2-dimethyl-3-hydroxyundec-8en-5-one (entry 4 ) is shown in figure 25 (page 114) with an enlargement of the spectral window from $\delta 45$ to $76 \mathrm{ppm}$. The most notable change in the chemical environment of the two diastereomers is reflected by the chemical shifts of the newly formed methine (C3) carbons, at $\delta 75.0$ and $\delta 74.7 \mathrm{ppm}$, and the neighbouring methylene (C-4) carbons, at $\delta$ 46.5 and $\delta 46.2 \mathrm{ppm}$. However, when synthesising 7,11-O-diphenylsilanediyl-2,2dimethyl3-hydroxyundec-8-en-5-one 65d (entry 9), only one diastereomer was observed by ${ }^{13} \mathrm{C}$ NMR. The spectrum of $\mathbf{6 5} \mathbf{d}$ is shown in figure 26 (page 115) with an expansion of the spectral window from $\delta 45$ to $\delta 76$ ppm clearly indicating that only one diastereomer is present. The analogous chemical shifts of the C-3 and C-4 carbons of $\mathbf{6 5 d}$ are at $\delta 74.8$ and $\delta 46.1$ ppm respectively.

Given that the isopropyl and phenyl substituents on the silyl tether are of comparable size, it was apparent that the size of the phenyl substituents was not primarily responsible for the high levels of asymmetric induction. Furthermore, by comparing entries 7 and 8, it was evident that the chiral ligand was not responsible for the observed asymmetric induction. Thus, one could conclude that the orbital interactions between the phenyl group and the enolate were responsible for the observed stereochemical induction. However, before this statement could be made, we first needed to determine that the anti adduct had indeed been synthesised. ${ }^{\mathrm{x} I I I}$

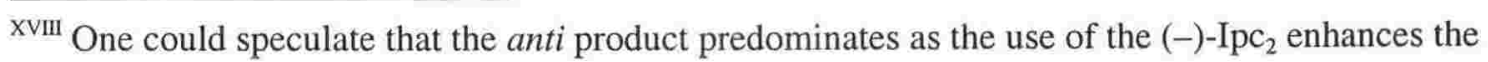
proportion of the anti product 


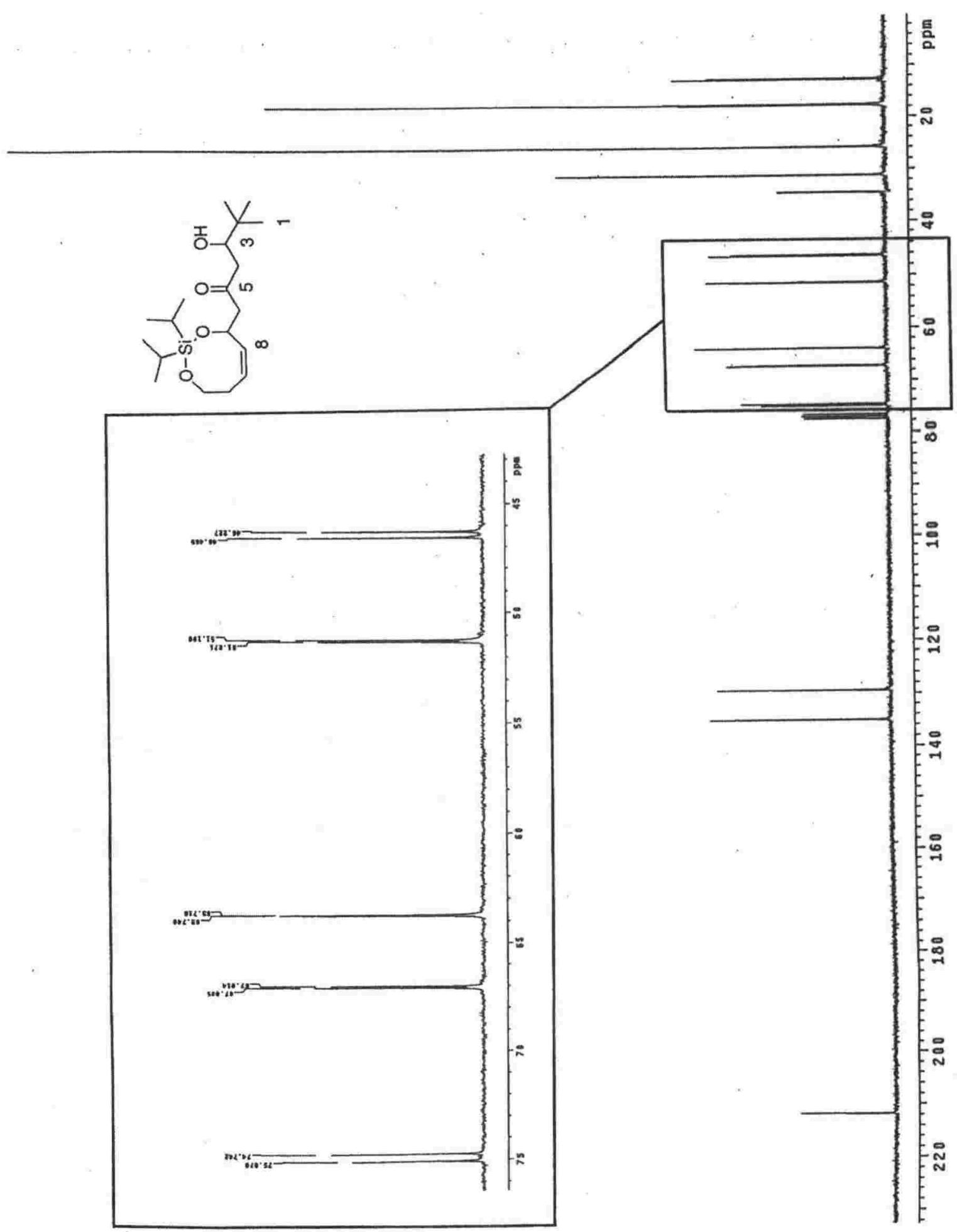

Figure 25: ${ }^{13} \mathrm{C}$ NMR of racemic 7,11-O-diisopropylsilanediyl-2,2-dimethyl-3-hydroxyundec-8-en-5-one 


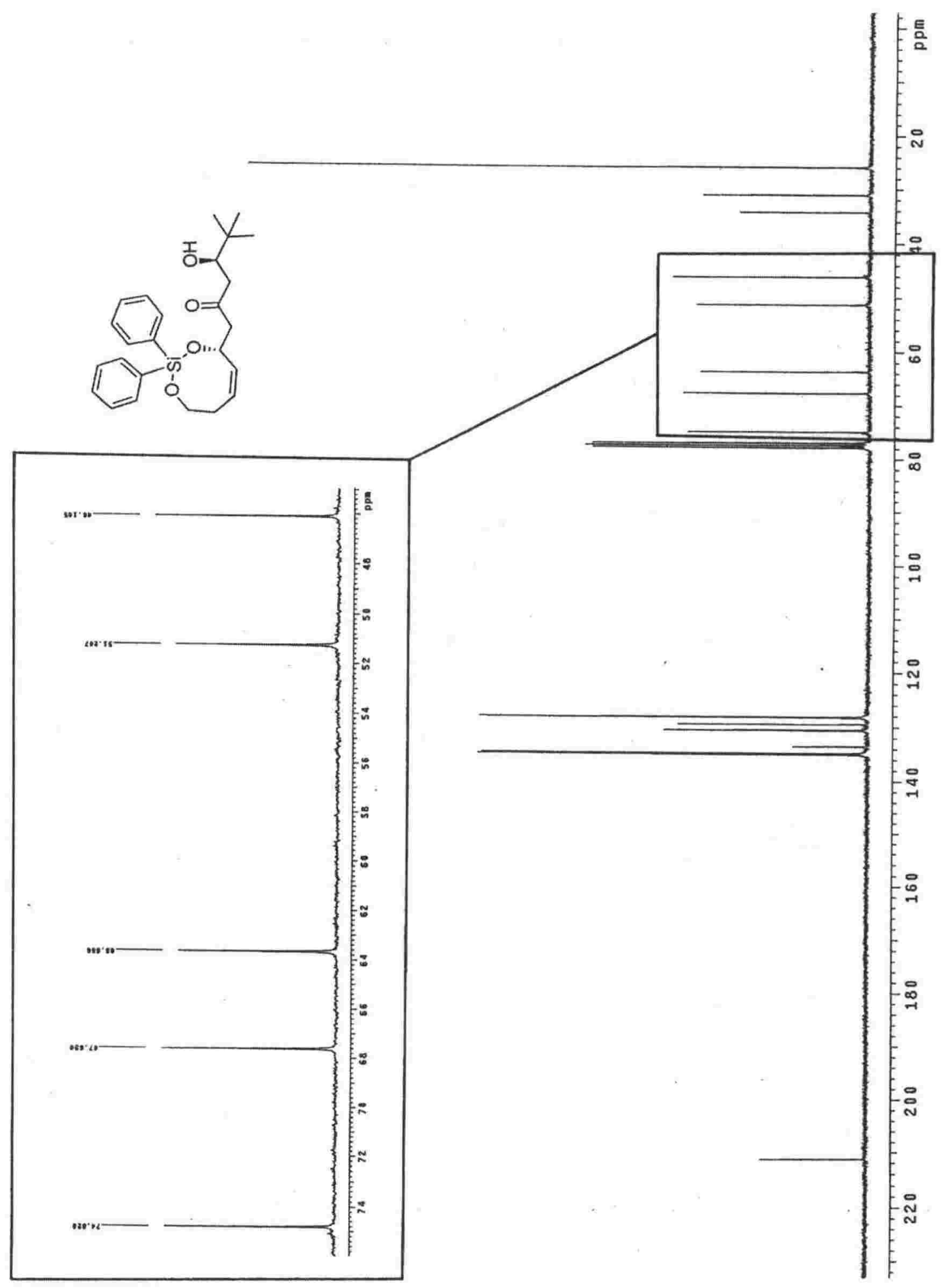

Figure 25: ${ }^{13} \mathrm{C}$ NMR of 7,11-O-diphenylsilanediyl-2,2-dimethyl-3-hydroxyundec-8-en5-one 


\subsection{4: Determination of the anti stereochemistry}

To determine the relative stereochemistry at C-3 of the aldol adduct, $92 \mathrm{c}$ was subjected to rigorous NMR analysis. A summary of the ${ }^{1} \mathrm{H},{ }^{13} \mathrm{C}, \mathrm{COSY}$ and nOe NMR spectral data for this substrate (recorded in $\mathrm{CDCl}_{3}$ ) is provided in table 5 , with the ${ }^{1} \mathrm{H}$ and ${ }^{13} \mathrm{C}$ spectra given in figures 27 and 28 , respectively.

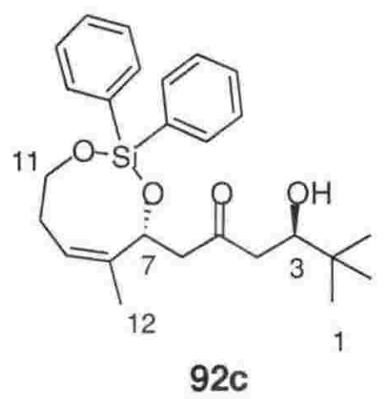

Table 5: NMR data for $92 \mathrm{c}$

\begin{tabular}{llllll}
\hline Position & $\boldsymbol{\delta}^{13} \mathrm{C}^{\mathrm{a}}$ & & $\boldsymbol{\delta}^{1} \mathbf{H}$ & COSY & nOe \\
\hline${ }^{\mathrm{T}} \mathrm{Bu}(1)$ & 25.9 & & $0.89(\mathrm{~s}, 3 \mathrm{H})$ & - & $4 \mathrm{a}, 4 \mathrm{~b}$ \\
${ }^{\mathrm{t}} \mathrm{Bu}(2)$ & 34.3 & $\mathrm{C}$ & - & - & - \\
3 & 74.8 & $\mathrm{CH}$ & $3.78(\mathrm{dd}, J=10.5,1.8 \mathrm{~Hz}, 1 \mathrm{H})$ & $4 \mathrm{a}, 4 \mathrm{~b}$ & - \\
$4 \mathrm{a}$ & 46.1 & $\mathrm{CH}_{2}$ & $2.58(\mathrm{dd}, J=17.7,10.2 \mathrm{~Hz}, 1 \mathrm{H})$ & $3,4 \mathrm{~b}$ & $1,6 \mathrm{a}$ \\
$4 \mathrm{~b}$ & - & $\mathrm{CH}_{2}$ & $2.76(\mathrm{dd}, J=17.8,2.0 \mathrm{~Hz}, 1 \mathrm{H})$ & $3,4 \mathrm{a}$ & $1,6 \mathrm{~b}$ \\
5 & 211.5 & $\mathrm{C}$ & - & - & - \\
$6 \mathrm{a}$ & 49.5 & $\mathrm{CH}_{2}$ & $2.99(\mathrm{dd}, J=14.9,9.8 \mathrm{~Hz}, 1 \mathrm{H})$ & 7 & $4 \mathrm{a}$ \\
$6 \mathrm{~b}$ & - & $\mathrm{CH}_{2}$ & $2.62(\mathrm{dd}, J=14.9,3.7 \mathrm{~Hz}, 1 \mathrm{H})$ & 7 & 12 \\
7 & 71.9 & $\mathrm{CH}$ & $4.90(\mathrm{dd}, J=9.8,3.3 \mathrm{~Hz}, 1 \mathrm{H})$ & $6 \mathrm{a}, 6 \mathrm{~b}$ & - \\
8 & 125.1 & $\mathrm{C}$ & - & - & - \\
9 & 140.7 & $\mathrm{CH}$ & $5.42(\mathrm{t}, J=9.6 \mathrm{~Hz}, 1 \mathrm{H})$ & $10 \mathrm{a}, 10 \mathrm{~b}$ & - \\
$10 \mathrm{a}$ & 30.9 & $\mathrm{CH}_{2}$ & $2.28(\mathrm{~m}, 1 \mathrm{H})$ & $10 \mathrm{~b}, 11$ & - \\
$10 \mathrm{~b}$ & - & $\mathrm{CH}_{2}$ & $2.91(\mathrm{~m}, 1 \mathrm{H})$ & $10 \mathrm{a}, 11$ & - \\
11 & 64.3 & $\mathrm{CH}_{2}$ & $4.05(\mathrm{~m}, 2 \mathrm{H})$ & $10 \mathrm{a}, 10 \mathrm{~b}$ & - \\
$12(\mathrm{Me})$ & 21.5 & $\mathrm{CH}_{3}$ & $1.60(\mathrm{~s}, 3 \mathrm{H}, 1 \mathrm{H})$ & - & 3 \\
\hline
\end{tabular}

${ }^{a}$ Multiplicity determined by HSQC Dept 


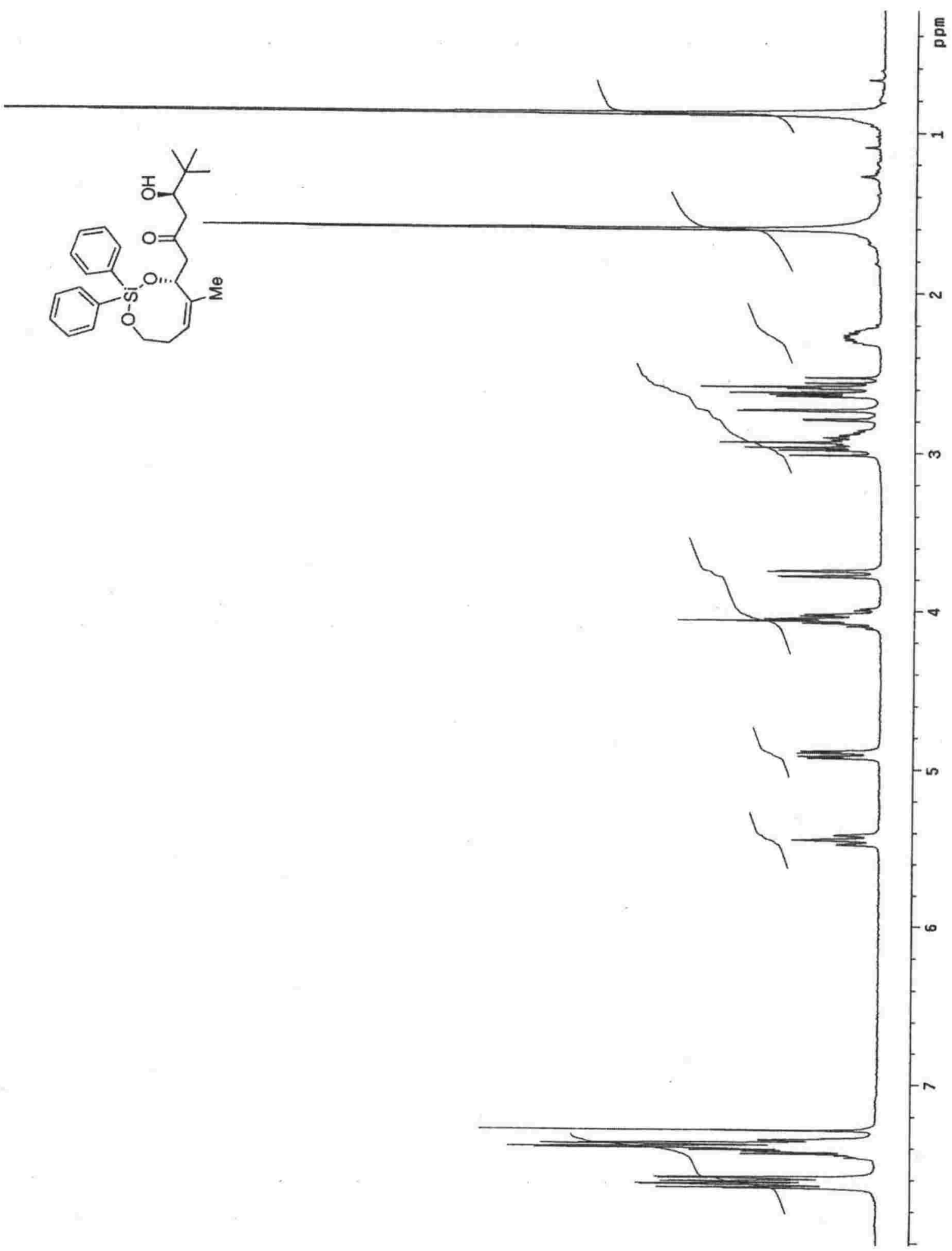

Figure $27:{ }^{1} \mathrm{H}$ NMR spectrum of $(3 R, 7 R, 8 Z)-7,11-O$-diphenylsilanediyl-2,2,8trimethylundec-8-en-5-one 


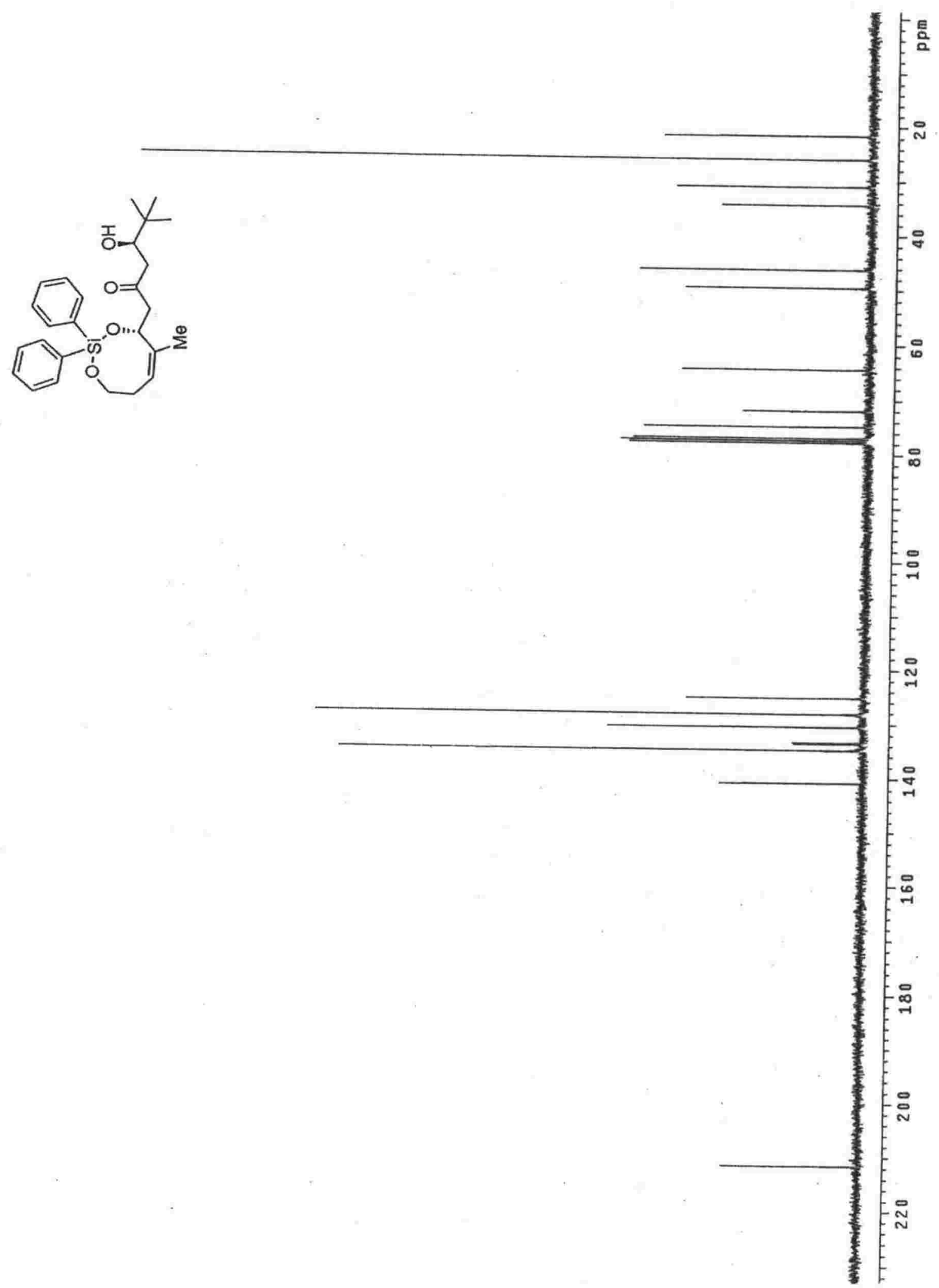

Figure 27: ${ }^{13} \mathrm{C}$ NMR spectrum of $(3 R, 7 R, 8 Z)-7,11-O$-diphenylsilanediyl-2,2,8trimethylundec-8-en-5-one 
The starting point for determining the stereochemistry at C-3, was determining the relative orientations of $\mathrm{H}-6 \mathrm{a}$ and $\mathrm{H}-6 \mathrm{~b}$, figure 28 . The large trans coupling of $9.8 \mathrm{~Hz}$ between H-7 and H-6a enabled H-6b to be differentiated, with H-6b being on the front face of the molecule as shown. The difference in the chemical environment of H-6a and $\mathrm{H}-6 \mathrm{~b}$ also indicated that there was restricted movement of the C-6 to C-8 carbon framework. Similarly, the trans coupling of $10.2 \mathrm{~Hz}$ between $\mathrm{H}-3$ and H-4a enables H-4a and $\mathrm{H}-4 \mathrm{~b}$ to be differentiated.

The crucial part in this analysis, however, was determining the conformation of the C-1 to $\mathrm{C}-5$ carbon framework. NOe's between $\mathrm{H}-1$ ('Bu) and both $\mathrm{H}-4 \mathrm{a}$ and $\mathrm{H}-4 \mathrm{~b}$ indicated that the ' $\mathrm{Bu}$ group bissects both $\mathrm{H}-4 \mathrm{a}$ and $\mathrm{H}-4 \mathrm{~b}$, yet did not distinguish between the geometry shown below or a more linear conformation with the side chain extended in a zig-zag fashion from C-5. This information was obtained from the nOe correlations between H-12 (Me) and H-6a and 3. NOes between H-12 and H-3 indicated that the C-1 to C-5 framework adopts the conformation shown below - these nOes would not be observed if a more linear conformation was favoured. NOes between C-12 and H-6b enabled us to determine that the methyl group $(\mathrm{H}-12)$ was sitting on the front face of the compound, as depicted. Thus, if the methyl substituent is on the front face of the molecule and nOes are observed between $\mathrm{C}-12$ and $\mathrm{H}-3$, then $\mathrm{H}-3$ must be on the front face of the molecule, with the hydroxyl behind. This corresponds to the anticipated 1,5-anti relationship. This analysis is further supported by the nOe between $\mathrm{H}-6 \mathrm{a}$ and $\mathrm{H}$ $4 \mathrm{a}$.

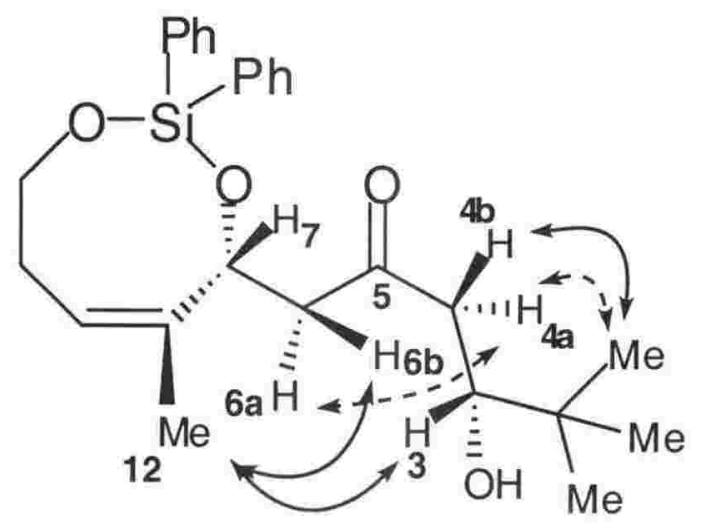

Figure 29: NOe correlations of $(3 R, 7 R, 8 Z)-7,11-O$-diphenylsilanediyl-3-hydroxy-2,2,8trimethylundec-8-en-5-one 
Although it was impossible to perform the same spectral analysis of $4,8-O$ diphenylsilanediyl-5-octen-2-one $\mathbf{9 2 d}$ due to the absence of the methyl substituent, by analogy we assumed that the 1,5-anti diastereomer was preferentially formed. In any case, a satisfactory strategy for the synthesis of the C-12 to C-24 fragment of pel A and its asymmetric coupling to the pyranose ring had been developed. Gratifyingly, this boron mediated 1,5-anti aldol reaction proceeded in both excellent yield and with excellent diastereomeric selectivity.

To provide further evidence for the involvement of $\pi$ stacking in the observed 1,5anti induction, modelling studies on the cyclic substrates were performed. ${ }^{\mathrm{XIX}}$ The methyl enol of ethers of the cyclic isopropyl derivative I and phenyl derivative II were modelled, figure 30. As seen, excellent overlap between the enol ether and a phenyl ring is occurring II, while with the isopropyl silanes I no interaction is available and the enol ether adopts an open geometry. Consequently, this results in $\pi$-facial discrimination for the phenyl substituents, while the open geometry of the isopropyl silyl derivatives is conducive to the approach of the aldehyde from either face.
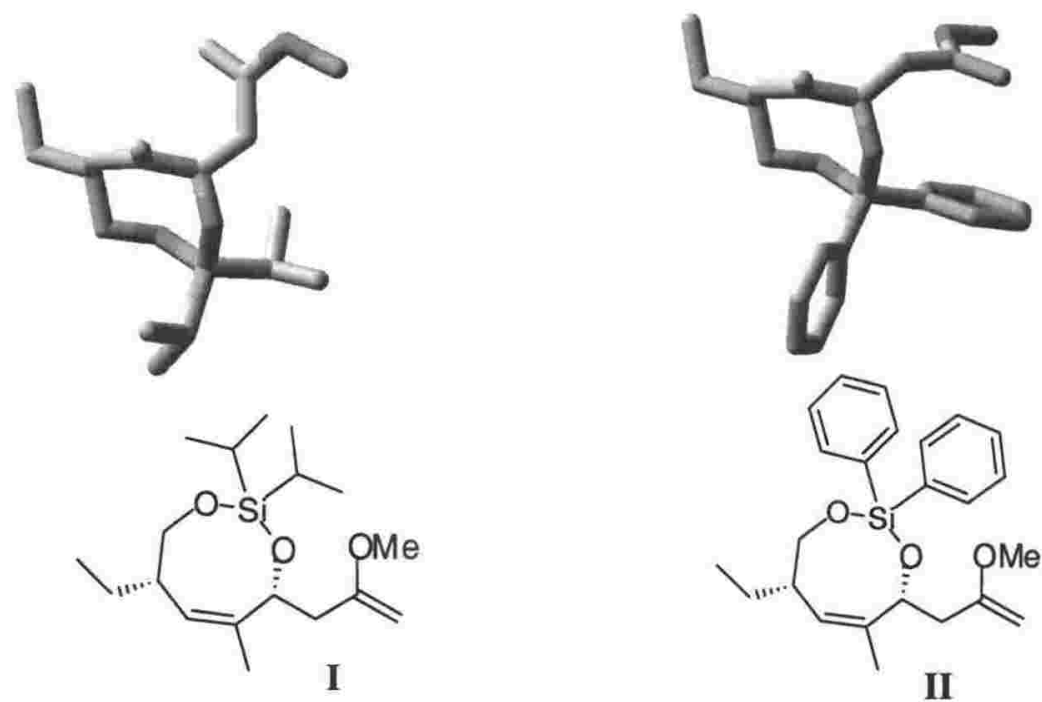

Figure 29: Molecular modelling of ${ }^{\mathrm{i}} \mathrm{Pr}_{2} \mathrm{Si}$ and $\mathrm{Ph}_{2} \mathrm{Si}$ enol ethers

\footnotetext{
${ }^{\mathrm{xIx}}$ Courtesy of Dr Paul Teesdale-Spittle, SBS, Victoria University of Wellington. Molecular models were energy minimized using the OPLSAA forcefield as implemented in Macromodel v7.2 using the Maestro v4.1 interface, and the lowest energy structures retained.
} 


\subsection{5: Putting the pieces of the puzzle together}

The high diastereoselectivity observed in the above experiments certainly justifies our hypothesis about the importance of $\pi$-stacking in certain boron-mediated 1,5-anti aldol reactions. The selectivities observed with entries 7-9 in table 7 are not due to electronic properties but are a result of the enolate being fixed in a conformation conducive to interaction with a single phenyl ring. These observations, however, raised some puzzling issues. If $\pi$-stacking interactions are responsible for the aforementioned 1,5-anti induction, could similar interactions be responsible for the previously reported 1,5-anti induction observed in acyclic systems where a para-methoxy benzyl substituent is placed at the B-position? Theoretically, a transition state, such as that depicted in scheme 83 , would account for the high 1,5-anti stereoselectivity observed. Similarly, if the transition state depicted below is responsible for the high degree of asymmetric induction for the PMB derivatives, a similar transition state could be proposed for the acyclic phenyl derivatives 66. Theoretically, these transition states could also involve aromatic-enolate interactions. Yet, referring back to table 4, entry 3 (pg 112) it is apparent that only nominal 1,5 asymmetric induction is observed in these cases.

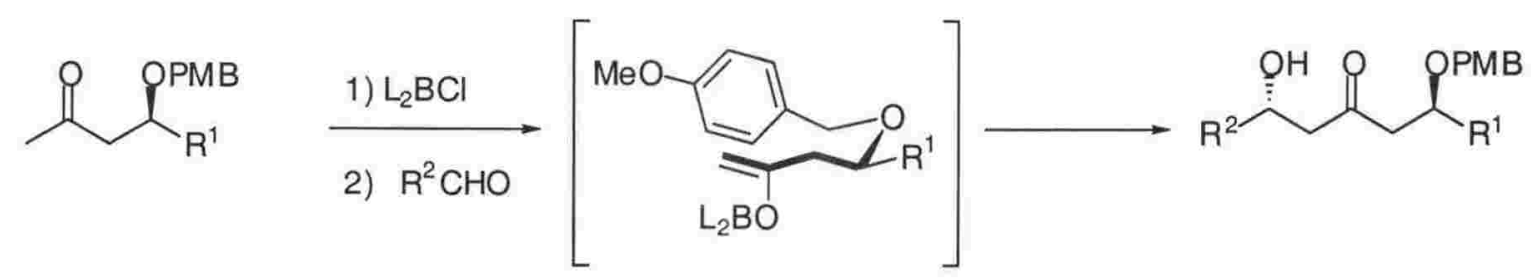

Scheme 83: Proposed conformation for the boron mediated 1,5-anti aldol reaction with substrates containing the PMB protecting group

After some consideration, it was proposed that the key difference between the above PMB mediated inductive asymmetric aldol reaction and the non-inductive reactions with the acyclic diphenyl sustituents $\mathbf{6 6}$ was the possibility of different transition states for the two aforementioned derivatives. By incorporating two phenyl moieties on a flexible 
acyclic tether, two transition state conformations are favourable for the acyclic phenyl derivative, scheme 84 .

The equatorial orientation of the olefinic side chain in conformation $\mathbf{I}$, would initially appear to favour conformation I over II. However, there may be some $\pi$-stacking interactions between this olefinic side chain and an aromatic ring, as depicted in II. In any case, having two phenyl substituents on the acyclic tether results in the possibility of at least two seemingly favourable conformers, resulting in negligible $\pi$-facial discrimination.
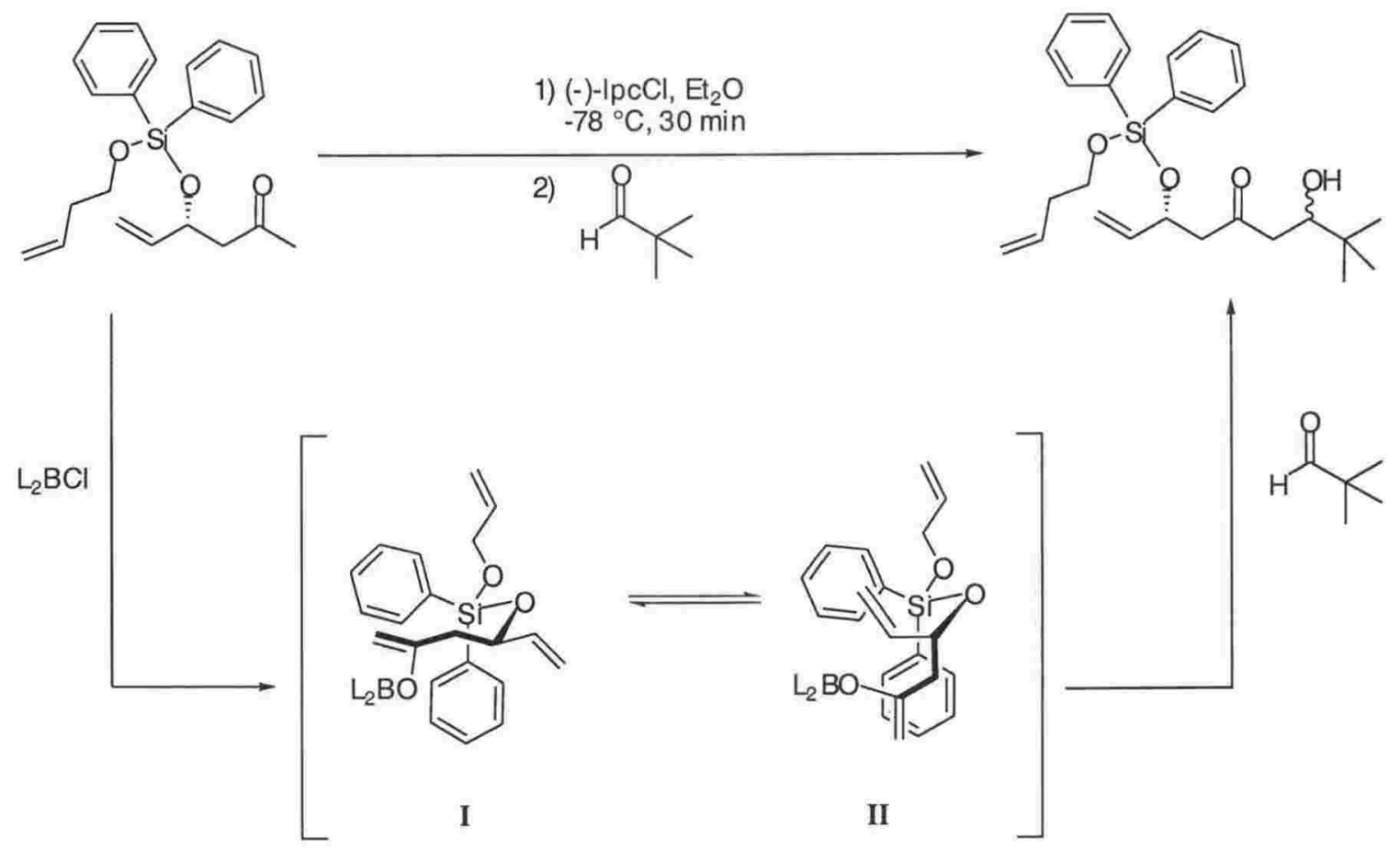

Scheme 84: Proposed conformations for the acyclic phenyl derivatives

Although some answers to the origin of 1,5-anti asymmetric induction had been gained, much still remained to adequately explain the inductive effects in other systems. We could confidently state that it is not the frequently reported electrostatic effects of the ß-oxygen that is responsible for these observations, however the exact influence of many 
of the B-hydroxy protecting groups was still unexplained. With the view that aromatic $\pi$ interactions must be at least partially responsible for the asymmetric 1,5-anti induction in acyclic systems further studies into the origin of the selectivity in boron mediated1,5-anti aldol reactions were conducted. These studies are discussed in the subsequent chapter. 


\section{Chapter 7}

\section{Studies into the origin of 1,5-anti induction in boron-mediated aldol reactions}

\section{1: Acyclic systems}

\subsection{1: The tert-butyl diphenyl silyl derivative}

As discussed in chapter 6, it was postulated that the reason for the poor diastereoselectivities observed with the diphenysilyl acyclic adduct (66b, pg 112) could be accounted for by the propensity of the substrate to exist in two conformers, as reviewed below. Although one would normally expect conformer $\mathbf{I}$ to be more favourable due to the equatorial orientation of the vinyl substituent, conformer II is simply a chain flip that may also result in additional $\pi$-stacking interactions.
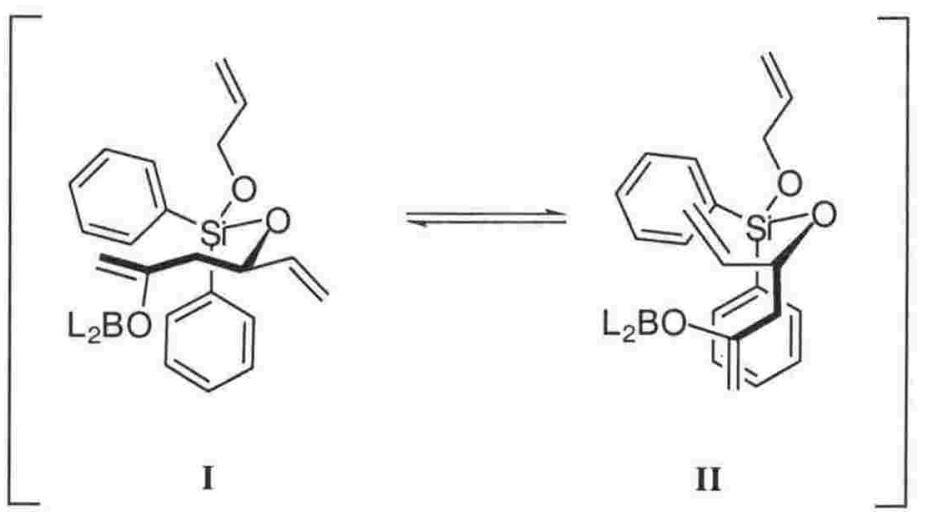

To clarify these results it was proposed that some additional aldol studies without the olefinic substituents were required. This would enable us to determine the influence, if any, of these substituents on the proposed conformations. The simplest way of conducting these reactions was to synthesise $\beta$-hydroxyketones and subsequently protect 
them with a tert-butyldiphenylsilyl substituent. Although the boron-enolates could again adopt the conformations as shown in figure 31 , the influence of the alkyl substituent, $\mathrm{R}^{1}$, may favour one conformer over the other and lead to differences in the diastereoselectivities. It was hoped that the larger, $\mathrm{R}^{1}$ substituents, such as the isopropyl or tert-butyl derivative would favour conformation I, leading to enhanced diastereoselectivies.
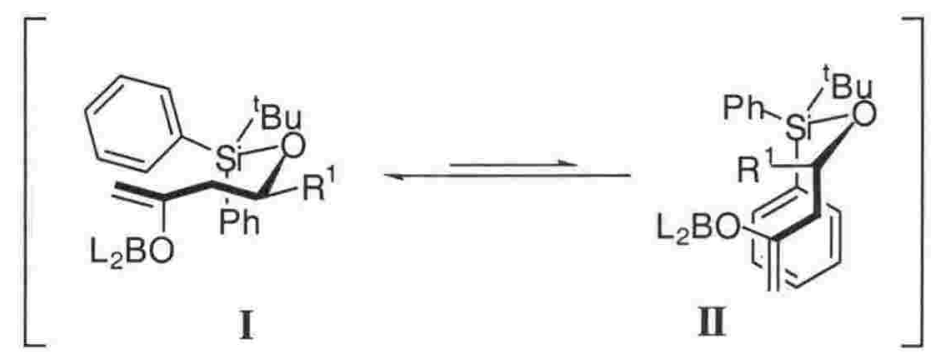

Figure 31: Possible conformations for the tert-butyldiphenylsilyl boronenol ether

Thus List' ${ }^{176 a, b}$ procedure was once again used for the synthesis of the requisite Bhydroxy ketones $\mathbf{9 4}$, which were subsequently protected as the tert-butyldiphenylsilyl derivatives 95 using standard silylation techniques, scheme 85 . Unfortunately, the most desirable adduct, where $\mathrm{R}^{1}=$ tert-butyl, could not be synthesised. Numerous attempts were made at protecting the tert-butyl B-hydroxy precursor, $94 \mathbf{b}$, but with little avail. Standard silylation techniques, as highlighted below, resulted in the isolation of starting materials, even after extended reaction periods. Even the combination of pyridine, $\mathrm{AgNO}_{3}$ and tert-butyldiphenylsilylchloride, ${ }^{185}$ which is believed to enhance silylation reactions via the formation of the intermediate silyl nitrate, ${ }^{186}$ primarily resulted in the reisolation of the starting $\beta$-hydroxy ketone. As anticipated, the use of one equivalent of potassium hydride as a base, along with catalytic amounts of the chelating macrocycle 18-Crown-6, and one equivalent of tert-butyldiphenylsilyl chloride resulted in decomposition of the starting material. ${ }^{186}$ 


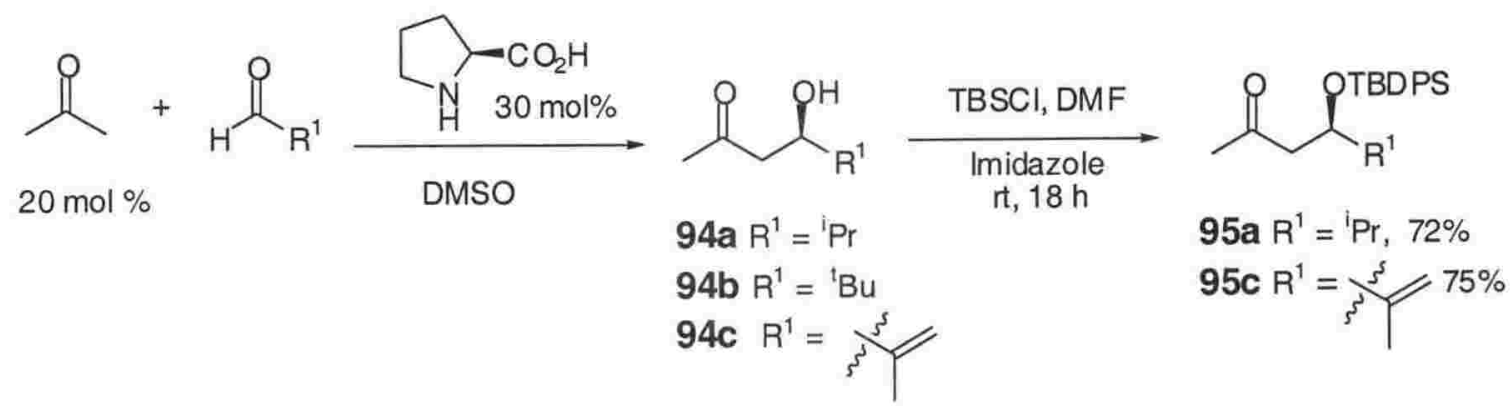

Scheme 85: Synthesis of the tert-butyldiphenyl silyl protected B-hydroxyl ketones

Nevertheless, with the suitable silylated derivatives 95a and 95c in hand, a selection of $\mathrm{Cy}_{2} \mathrm{BCl}$ mediated aldol studies were conducted, scheme 86 . Unfortunately, the alkyl substituent $\left(\mathrm{R}^{1}\right)$ appeared to have little influence on the diastereoselectivites of these reactions with ratios from $2: 1$ to $2.5: 1$ being observed, table 6 . By using both isopropylaldehyde and pivaldehyde as the aldehyde acceptor, we were able to establish that the aldehyde had only a limited effect on the observed diastereoselectivities. ${ }^{187}$

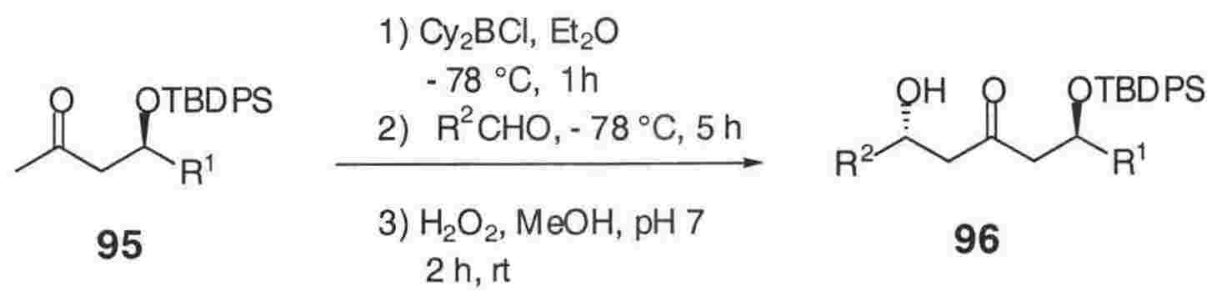

Scheme 86: Aldol studies with the tert-butyldiphenylsilyl derivatives

Table 6: Boron mediated aldol studies with the tert-butyldiphenylsilyl derivatives

\begin{tabular}{lllll}
\hline Product & $\mathbf{R}^{1}$ & $\mathbf{R}^{2}$ & Yield & Diastereoselectivity $^{\mathbf{a}}$ \\
\hline 96a & $i \mathrm{Pr}$ & $i \mathrm{Pr}$ & $88 \%$ & $2: 1$ \\
$\mathbf{9 6 b}$ & $i \mathrm{Pr}$ & $t \mathrm{Bu}$ & $86 \%$ & $2.5: 1$ \\
$96 \mathbf{c}$ & & $89 \%$ & $2: 1$ \\
& & & \\
\hline
\end{tabular}

${ }^{2}$ Diastereoselectivities determined by ${ }^{13} \mathrm{C}$ NMR analysis of the crude substrate 
Thus it was apparent that the interconversion of the two proposed conformations was a facile process. Consequently, a substituent with only one aromatic ring was required as the $\beta$-hydroxy protecting group.

\subsection{2: The dimethylphenyl silyl derivative}

To eliminate the possibility of having two favourable conformations, it was proposed that the analogous dimethylphenylsilyl derivative would more suitable at the Bposition. Thus, the B-hydroxy ketones 94 were protected as the dimethylphenyl silyl derivatives, scheme 87.

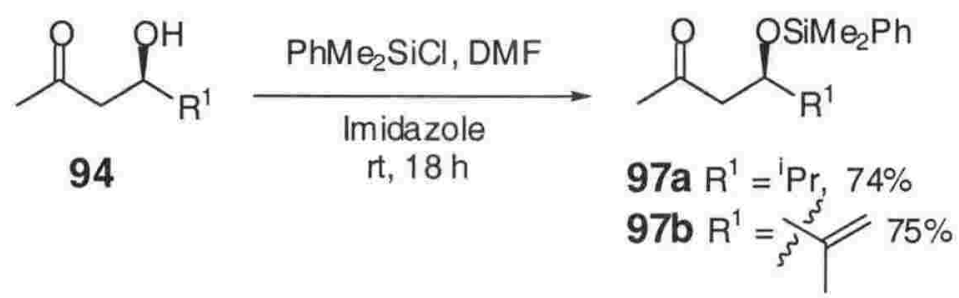

Scheme 87: Synthesis of the dimethylphenylsilyl derivatives

A selection of $\mathrm{Cy}_{2} \mathrm{BCl}$ mediated aldol reactions were then conducted with the dimethylphenyl silyl derivatives $\mathbf{9 7}$, scheme 88 . It was proposed that these silyl derivatives would participate in the boron mediated aldol reactions by adopting the conformation shown below to maximise $\pi-\pi$ interactions. Such interactions would result in the desired 1,5-anti adducts $\mathbf{9 8}$.

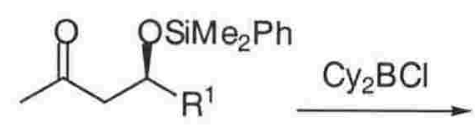

97
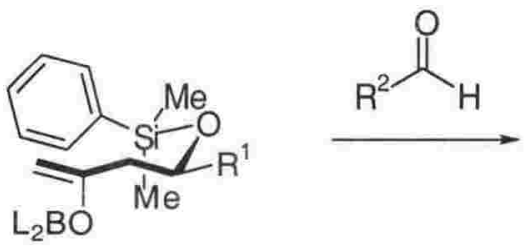<smiles>[R]C(O)CC(=O)CC([R16])OCc1ccccc1</smiles>

98

Scheme 88: Aldol studies with the dimethylphenylsilyl derivatives 
Although the yields for these reactions were good, exceptionally poor diastereoselectivities were observed, table 7 . Obviously $\pi$-stacking interactions were not involved in these particular transition states.

Table 7: Boron mediated aldol studies with the dimethylphenylsilyl derivatives

\begin{tabular}{lllll}
\hline Product & $\mathbf{R}^{1}$ & $\mathbf{R}^{2}$ & Yield & Diastereoselectivity $^{\mathrm{a}}$ \\
\hline $\mathbf{9 8 a}$ & ${ }^{\mathrm{i}} \mathrm{Pr}$ & ${ }^{\mathrm{t}} \mathrm{Bu}$ & $80 \%$ & $1.1: 1$ \\
$\mathbf{9 8 b}$ & & $82 \%$ & $1.1: 1$ \\
& & & & \\
\hline
\end{tabular}

${ }^{2}$ Diastereoselectivities determined by ${ }^{13} \mathrm{C}$ NMR analysis of the crude product

The lack of asymmetric induction in these aldol studies was initially particularly puzzling. However upon closely reviewing the literature, it became apparent that the PMB ether was used exclusively for good 1,5-anti induction in acyclic systems, suggesting that an electron-donating group is required for effective asymmetric induction and that a benzyl ether would be less effective. To investigate this supposition, the appropriately protected benzyl derivative was synthesised.

\subsection{3: The benzyl ether as a protecting group}

Synthesis of the requisite benzyl derivative began with the benzylation of $(R)-5$ methyl-4-hydroxylhexan-2-one using the previously described benzyl imidate methodology. ${ }^{153}$ This reaction proceeded smoothly, giving the desired benzylated adduct 99 in a modest $60 \%$ yield, scheme 89 . A boron-mediated aldol reaction with pivaldehyde was then conducted and, as anticipated, only moderate diastereoselectivity was observed. The ratio of diastereomers, determined to be $3: 1$ by ${ }^{13} \mathrm{C}$ NMR analysis, was significantly inferior to the almost exclusive ratios observed for the para-methoxy benzyl derivatives. 


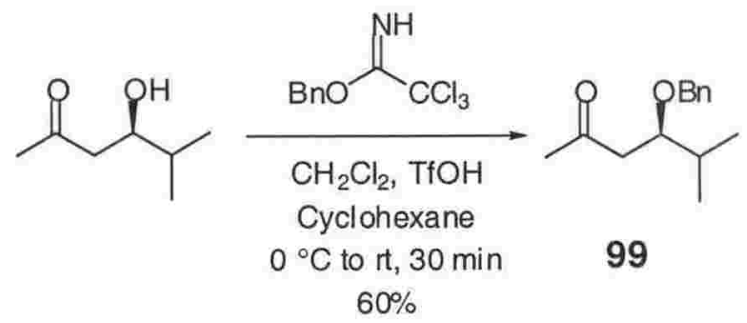

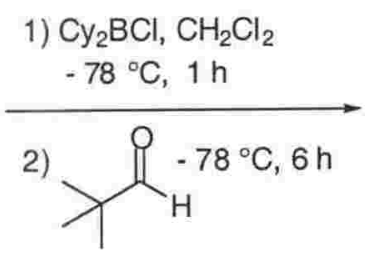

3) $\mathrm{H}_{2} \mathrm{O}_{2}, \mathrm{MeOH}, \mathrm{pH} 7$ $2 \mathrm{~h}, \mathrm{rt}, 74 \%$

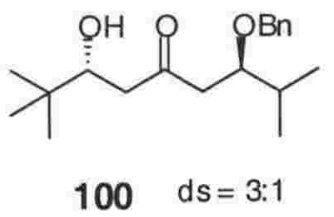

$100 \mathrm{ds}=3: 1$

Scheme 89: Aldol study with the benzyl protected derivative

As the steric size of the paramethoxy benzyl and the benzyl derivatives are comparable, this suggests that it is not a steric factor that is responsible for the differing levels of asymmetric induction observed for both derivatives. Rather, these results suggest that it is the electron donating ability of the OMe substituent that is crucial for efficient interaction between the enolate and aromatic substituent. It is a well renowned phenomenon that methoxy substituents donate electron density to an aromatic ring, and by doing so, presumably increase the capacity of the aromatic ring to participate in $\pi$ stacking interactions. For example, the ${ }^{1} \mathrm{H}$ chemical shifts of the ring protons in anisole are 6.65 , 7.14 and $6.74 \mathrm{ppm}$ respectively, for the ortho, meta and para postitions, compared to the ${ }^{1} \mathrm{H}$ chemical shift of 7.37 for benzene, ${ }^{188}$ showing that these protons are shielded by an increase in electron density.

An example of a subtle change to an electronic environment and its effect on $\pi$ stacking interactions is the fluorescent quenching abilities of the glyoxylates 101 and $102 .{ }^{189} \mathrm{By}$ removing the cyclohexyl moiety to give derivative $\mathbf{1 0 2}$, intramolecular quenching for this derivative proved to be even more efficient. Less rigidly defined conformations, such as that of glyoxylate $\mathbf{1 0 2}$, are particularly sensitive to subtle changes in their electronic environment. ${ }^{179}$ We observed a similar phenomenon for the flexible, acyclic benzyl 99 and the PMB derivatives (scheme 80, pg 107), as evidenced by a difference in the diastereoselectivities of the analogous aldol reactions.

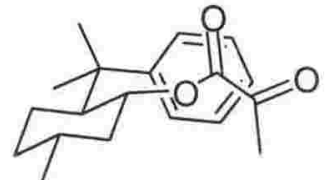

101

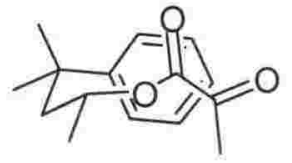

102 


\subsection{5: Conclusions}

The above results suggest that these boron mediated 1,5-anti aldol reactions are particularly sensitive to slight changes in the substituent at the B-position. If subtle $\pi$ stacking interactions are responsible for the differences between the benzyl and PMB derivatives, then the proposed transition state will also be influenced by other subtle changes to the $B$-substituent. Indeed, by considering the conformers $\mathbf{A}$ and $\mathbf{B}$ for the previously discussed silyl derivatives, one can see how the phenyl, or methyl, substituents on the silyl tethers affect the diastereoselectivities. In both $\mathbf{A}$ and $\mathbf{B}$, unfavourable 1,3diaxial interactions between the methyl, or phenyl, substituent on the silyl tether, the axial $\mathrm{H}$ (as depicted) and the boron enolate oxygen results in a less favourable, higher energy, conformation. Consequently, low diastereoselectivities are observed for the subsequent aldol reactions.

This proposition is further verified by an additional study whereby the analogous paramethoxyphenyldimethylsilyl substituted substrate $\mathbf{C}$ was synthesised and subjected to boron mediated aldol reactions, using $\mathrm{Cy}_{2} \mathrm{BCl}$ as the boron reagent. ${ }^{\mathrm{xx}}$ Again, poor diastereoselectivities, ranging from $1.2: 1$ to $2: 1$ were observed. From this observation, two important conclusions can be made. First, it is not the steric influence of the OMe substituent that is somehow enhancing the diastereoselectivity for the previously reported PMB mediated 1,5-anti aldol reactions. Second, the presence of the methyl substituents significantly disfavours the proposed conformation C. Apparently these unfavourable 1,3-diaxial interactions cannot be overcome by an increase in the electron density of the aromatic ring.

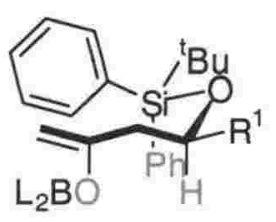

A

ds: $2: 1$ to $2.5: 1$

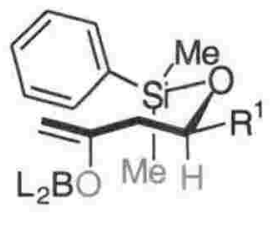

B

ds: 1.1:1

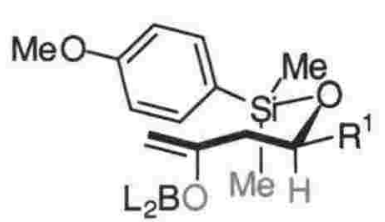

C

ds:1.2:1 to $2: 1$

\footnotetext{
${ }^{\mathrm{xx}}$ Results courtesy of Dr Y. Dong, Victoria University of Wellington
} 
Thus, in summarising the above information, a few general statements concerning boron mediated acyclic 1,5-anti aldol reactions can be made. An electron rich aromatic system is required at the B-position of the parent B-hydroxy ketone, conformation $\mathbf{D}$. Although moderate selectivity is observed with a benzyloxy substituent at the B-postion $\mathbf{E}$, diastereoselectivities are greatly enhanced by the electron rich, paramethoxybenzyl derivative. The steric effect of the paramethoxybenzyl substituent is not responsible for the high levels of 1,5-anti induction, as observed with the poor diastereoselectives obtained with the paramethoxydimethylsilyl derivative $\mathbf{C}$. Although the electron donating abilities of the paramethoxy substituent greatly enhance the diastereoselectivities for the PMB derivatives, the additional methyl substituents in $\mathbf{C}$, disfavour the formation of the $\pi$-stacked transition state and outweigh any additional positive influence of the methoxy substituent. However it is not solely these methyl substituents that result in decreased levels of 1,5-asymmetric induction, again compare aldol reactions with the $\mathrm{OBn}(\mathbf{E})$ and PMB (D) derivatives. Unfavourable 1,3-diaxial interactions and a relatively electron poor aromatic ring explains the poor diastereosectivites observed with the dimethylphenylsilyl $\mathbf{B}$ and tert-butyldiphenylsilyl A derivatives. The presence of two phenyl substituents in $\mathbf{A}$ also leads to two possible transition state conformations.

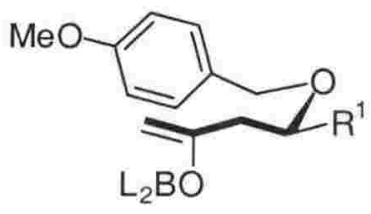

D

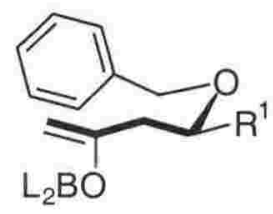

E

ds: $3: 1$

Thus to date, all of these observations are consistent with a model in which $\pi$-stacking interactions are responsible for the high degree of boron mediated 1,5-anti induction observed with the PMB derivatives. For effective $\pi$-stacking interactions, and hence efficient boron mediated 1,5-anti-induction, an electron rich aromatic system, attached to the $B$-oxygen via a methylene linkage, is required. 


\section{2: Cyclic systems}

\subsection{1: The pyranose system}

Next, we turned our attention to other cyclic systems, such as $\mathbf{1 0 3}$, in which excellent 1,5-anti induction has been observed, scheme 90. Kozmin reported selectivities of over 99:1 with pyrans of the type $\mathbf{1 0 3}$ where $\mathrm{R}^{1}=$ benzyl, and $\mathrm{R}^{2}=$ allyl. ${ }^{178} \mathrm{Kozmin}$ rationalized the high stereoselectivity to be a consequence of the B-alkoxy group and cited the studies conducted by Evans ${ }^{162}$ on alternative cyclic systems as precedence for this observation.<smiles>[R7]C1CC([R])OC(CC(C)=O)C1</smiles>

103
1) $\mathrm{L}_{2} \mathrm{BCl}$

2) $\mathrm{R}^{3} \mathrm{CHO}$<smiles>[R]C1CC([R7])OC(CC(=O)CC(O)O)C1</smiles>

104

Scheme 90: Boron mediated1,5-anti aldol studies in cyclic systems

However, due to the presence of benzyl substituent $\mathrm{R}^{1}$, we proposed that a conformation such as $\mathbf{I}$, where $\mathrm{R}^{1}=$ allyl, $\mathrm{R}^{2}=\mathrm{OBn}$, in figure 32 , was responsible for the high diastereoselectivity. Our initial impression was that $\pi$-stacking interactions were responsible for this preferred conformation, however upon closer examination, the intramolecular distance of approximately $7.5 \AA$ between the benzyl substituent and the boron enolate was not amenable to $\pi$ stacking. Thus, it appeared that with these systems, aldehyde facial discrimination was achieved by simple steric hinderance from the C-4 $\left(\mathrm{R}^{1}\right)$ substituent. This was confirmed by the molecular modelling of the methyl enol ether of $\mathbf{1 0 3}$ (where $\mathrm{R}^{1}=$ allyl, $\mathrm{R}^{2}=\mathrm{OBn}$ ), figure 32 . ${ }^{\mathrm{xx}}$

\footnotetext{
${ }^{\mathrm{XXI}}$ Courtesy of Dr Paul Teesdale Spittle, SBS, Victoria University of Wellington
} 


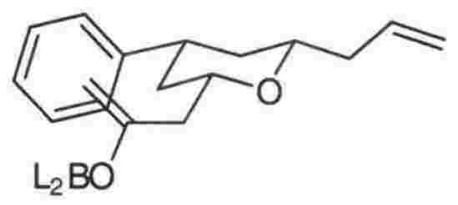

I

Figure 32: Proposed conformation for the boron enolate of $\mathbf{1 0 3}$
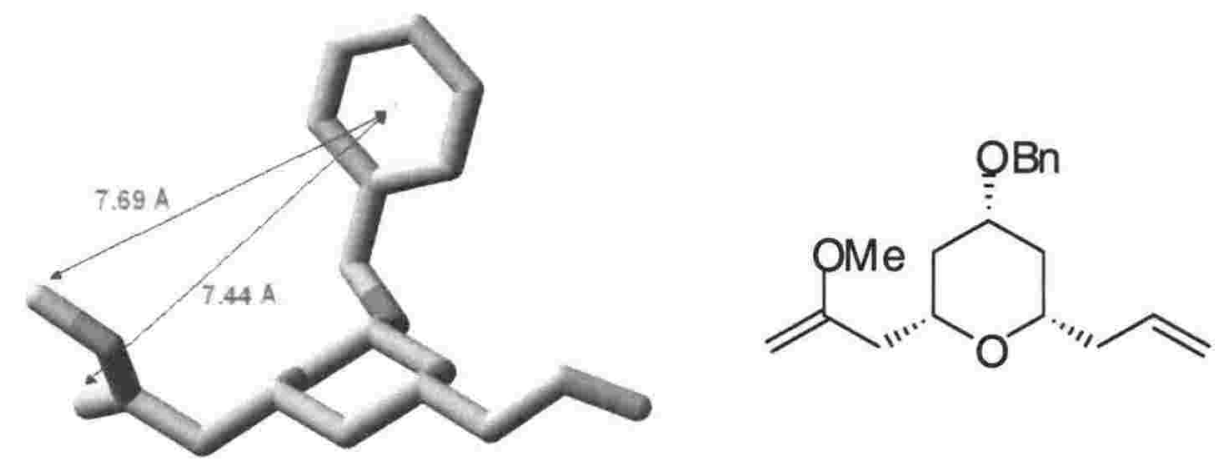

Figure 33: Molecular modelling of the methoxy enol ether of pyranose $\mathbf{1 0 3}$

To further verify this hypothesis, a similar cyclic derivative with a different substituent at C-4 was synthesised. In an attempt to simplify the system, a derivative where $\mathrm{R}^{2}=\mathrm{H}$, was made by the addition of the readily available but-3-en-1-ol to 4methoxybut-3-en-2-one, as depicted in scheme 91. Unfortunately the subsequent Prins rearrangement ${ }^{190}$ resulted in a $4: 1$ mixture of diastereomers. Presumably this diasteromeric mixture was a consequence of the absence of a $\mathrm{R}^{2}$ substituent (scheme 90), which would normally adopt an equatorial position to favour the intermediate chair transition state during the Prins rearrangement. 


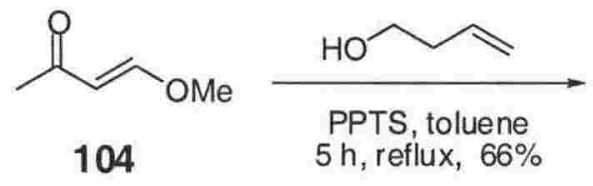

104<smiles>C=CCCO/C=C/C(C)=O</smiles>

1) TFA, $\mathrm{CH}_{2} \mathrm{Cl}_{2}$ $5^{\circ} \mathrm{C}, 48 \mathrm{~h}$

2) $\mathrm{LOH}, \mathrm{THF}, \mathrm{H}_{2} \mathrm{O}$ $10 \mathrm{~min}, 75 \%$<smiles>CC(=O)C[C@@H]1C[C@@H](O)CCO1</smiles>

Scheme 91: Attempted synthesis of aldol precursor 106

To avoid the added complications of accounting for the effects of the diastereomers during the subsequent aldol studies, the original synthesis, as reported by Kozmin was repeated, to give the intermediate pyranose adduct $\mathbf{1 0 8}$ in good diastereoselectivity, scheme 92. The free hydroxyl group was then protected, in excellent yield, with a TBS group to form the aldol precursor 109 , scheme 93.

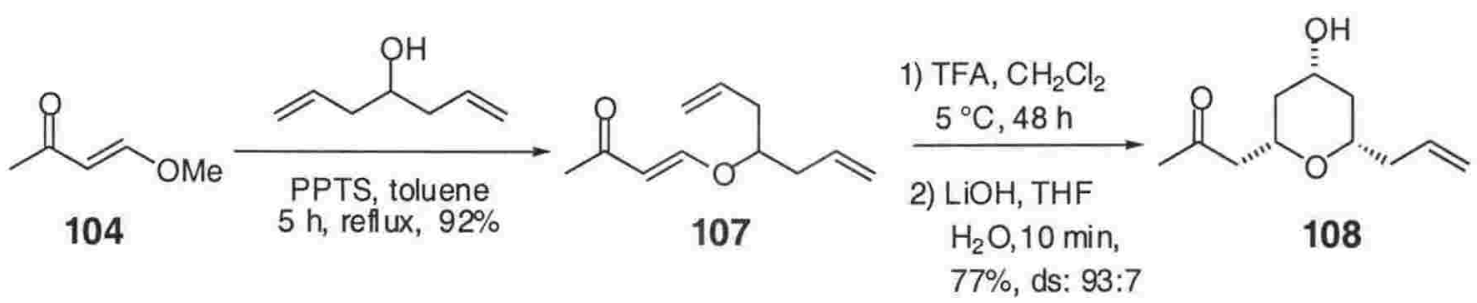

Scheme 92: Synthesis of the intermediate pyranose adduct $108^{178}$<smiles>C=CC[C@H]1C[C@@H](O)C[C@@H](CC(C)=O)O1</smiles>

108<smiles>C#CCC1CC(O)CC(CC(C)=O)O1</smiles>

108

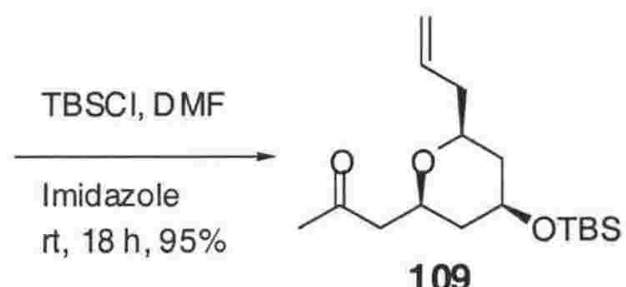

109

Scheme 93: Synthesis of novel aldol precursor 109

A boron mediated aldol reaction between 109 and isobutyraldehyde was then conducted, scheme 94, and as anticipated, excellent levels of diastereoselective induction were 
observed. ${ }^{\text {XII }}$ From these results, it can be verified that $\pi$-stacking interactions were not responsible for the observed high levels of diastereoselectivity in these cyclic systems, and it appeared that it was the steric influence of the substituent at C-4 that predominantly influenced the stereochemical outcome of these reactions. However, based on these studies alone, there was still not sufficient evidence to confidently make this proposition. An aldol study on a similar substrate without a C-4 substituent would be required to produce low levels of selectivity.<smiles>C=CC[C@H]1CC([OH2+])CC(CC(C)=O)O1</smiles>

109

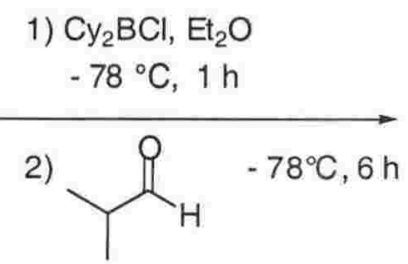

3) $\mathrm{H}_{2} \mathrm{O}_{2}, \mathrm{MeOH}, \mathrm{pH} 7$ $2 \mathrm{~h}, \mathrm{rt}, 81 \%$

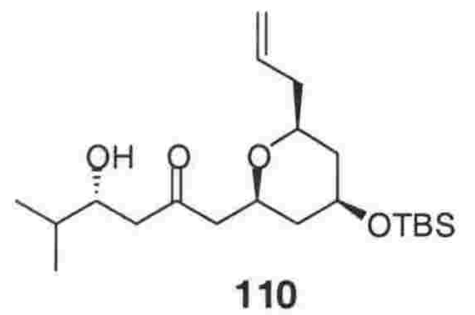

d.s $>99: 1$

Scheme 94: Boron mediated 1,5-anti aldol reaction with pyranose $\mathbf{1 0 9}$

Fortunately, a review of the literature revealed that such studies had already been conducted. During the synthesis of the C-1 to C-21 fragment of the dimeric polyketide natural product SCH 351448, De Brabander et. al. conducted a boron mediated aldol reaction using 111, scheme $95 .{ }^{191}$ Again, De Brabander cited studies by the Evans ${ }^{162 a}$ and Paterson ${ }^{163 a}$ groups as precedent for this 1,5-anti induction. However, in De Brabander's subsequent studies, only low to modest levels of diastereodifferentation were observed.

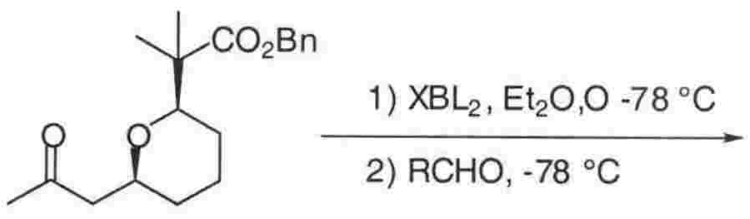

111

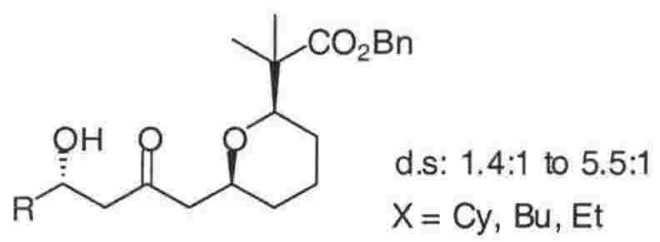

112

Scheme 95: Boron mediated 1,5-anti aldol studies conducted by De Brabander et. al.

$\overline{\mathrm{xXII}}$ Determined by ${ }^{13} \mathrm{C}$ NMR analysis of the crude product 
Although the intrinsic facial bias of the enolborinate partner was reported to be responsible for some of the more subtle differences in the levels of stereochemical induction for the different aldehyde partners, no explanation was given for the overall decreased levels of stereochemical induction when compared to the examples reported by Evans, Paterson, or Kozmin. Enhanced stereoselectivities in De Brabander's studies were only obtained after screening a variety of boron reagents with different ligands. However, after comparing our studies with those by Kozmin and De Brabander, it seemed apparent that the steric nature of the C-4 substituent greatly influences the degree of stereochemical induction. Where there is no appendage at C-4, as in De Brabander's case, poor diastereoselectives are observed.

This concept is illustrated by considering the conformation of the pyran ring and the proposed transition state shown in figure 34 . Here the enolate side chain adopts an equatorial orientation, with the larger boron substituent positioning itself furthest away from the cyclohexyl ring to minimise the electrostatic repulsive effects of the ring oxygen and the steric effects of the boron ligands. Where $\mathrm{R}^{1}$ is large, such as OTBS or OBn, the aldehyde preferentially approaches the enolate from the front face with the larger $\mathrm{R}^{3}$ substituent on the aldehyde adopting an equatorial position in the intermediate transition state. ${ }^{\mathrm{XXIII}}$ Such a transition state gives the desired anti- diastereoselectivity. A summary of the diastereoselectivities for the various $\mathrm{R}^{1}, \mathrm{R}^{2}$ and boron ligands $(\mathrm{L})$ is provided in Table 8 overleaf.
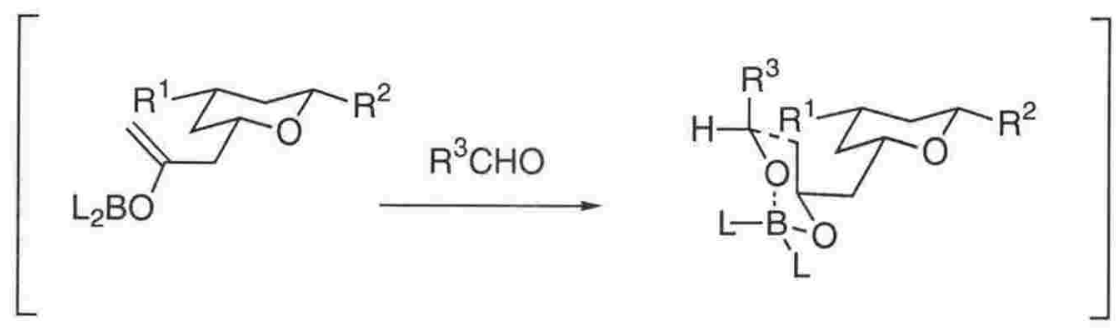

Figure 34: Proposed transition state for the boron-mediated aldol reaction

xxIII Due to the minimal energy difference between the chair and twist boat transition state, this transition state has been shown in the chair conformation as this is easier to depict pictorially and does not alter the stereochemical outcome 
Table 8: Boron mediated aldol studies with derivatives of pyranose 103

\begin{tabular}{|c|c|c|c|c|c|}
\hline $\mathbf{R}^{1}$ & $\mathbf{R}^{2}$ & $\mathbf{L}$ & RCHO & $\begin{array}{l}\text { Diastereoselectivity } \\
\text { (anti:syn) }\end{array}$ & Ref \\
\hline $\mathrm{OBn}$ & allyl & $\mathrm{Cy}$ & & $>99: 1$ & 178 \\
\hline OTBS & allyl & $\mathrm{Cy}$ & ${ }^{\mathrm{t}} \mathrm{BuCHO}$ & $>99: 1$ & 192 \\
\hline $\mathrm{H}$ & $\mathrm{CMe}_{2} \mathrm{CO}_{2} \mathrm{Bn}$ & $\mathrm{Cy}$ & EtCHO & $1.8: 1$ & 192 \\
\hline $\mathrm{H}$ & $\mathrm{CMe}_{2} \mathrm{CO}_{2} \mathrm{Bn}$ & Et & EtCHO & $5.5: 1$ & 192 \\
\hline
\end{tabular}

From this analysis, a lack of a substituent at C-4 clearly results in inherently lower diastereoselectivities as there is no $\mathrm{R}^{1}$ substituent to hinder the approach of the aldehyde from the back face. Thus, the analysis becomes a little more complicated. With no substituents to obstruct the approach of the aldehyde from the back face, it becomes more difficult to determine the relative energies of the both the anti and syn transition states. ${ }^{193}$ In these cases, the choice of ligands on the boron enolate will have a much greater influence on the preferred transition state, but without an in depth knowledge of the preferred transition state conformation, it is difficult to predict the stereochemical outcome of these aldol reactions.

\section{3: Boron mediated 1,5-anti induction - a summary}

From the studies discussed in this and the preceding chapter, some general observations concerning the origin of diastereoselectivity in boron mediated 1,5-anti aldol reactions can be made. Although the statement that these reactions are influenced by the B-hydroxy substituent' has not been disputed, it needs to be addressed with caution. A more in depth analysis of each system is required. Thus in general, we have identified and proposed transition states for three different systems that arise when conducting boron-mediated aldol reactions. These are summarised below: 
The judicious use of electron rich $\pi$ systems at the $\beta$-hydroxy protecting group is required for favourable $\pi$ interactions and hence selectivities in acyclic boron mediated 1,5-anti induction. Unfavourable 1,3-diaxial interactions are observed if there are substituents on the carbon, or silicon, linkage to these electron rich aromatic groups. These interactions greatly disfavour the formation of the proposed $\pi$-stacked transition state, resulting in negligible selectivities.

\section{ii) Rigid cyclic systems with favourable $\pi$-stacking interactions}

In some cyclic systems, such as that developed for the synthesis of pel $A$, the cyclic system is rigid enough to hold the aromatic ring in close proximity to the enolate. This leads to favourable $\pi$ stacking interactions, and hence selectivities.

iii) Cyclic systems where steric hinderance directs the approach of the aldehyde

The carbon framework of the cyclic system dictates the stereochemical outcome of the reaction. Such systems are more difficult to analyse and require careful consideration of the proposed transition states. 


\section{Chapter8:}

\section{Concluding Remarks}

Although we were unsuccessful in determining the absolute stereochemistry of peloruside A, presumably due to the small sample size or complex nature of this molecule, significant advancements have been made towards its synthesis. To-date, a successful strategy for the synthesis of the C-8 to C-24 framework has been developed. This carbon framework is depicted in figure 35 .

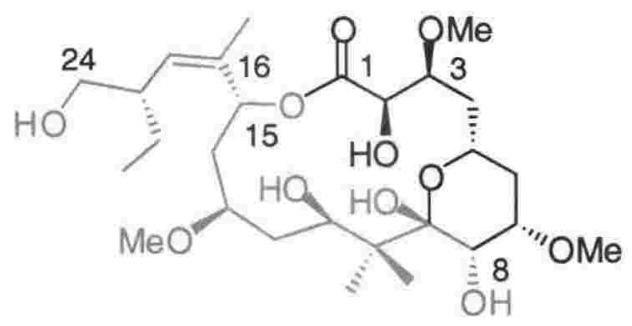

Figure 35: Peloruside A with the C-8 to C-24 framework highlighted

The synthesis of the C-8 to C-11 fragment proceeds smoothly and needs no further investigation. This five-step synthesis proceeds in good overall yield and has been successfully performed on the multigram scale.

A short, facile synthesis of the C-12 to C-24 fragment has also been developed. To date, the ethyl side chain has not been incorporated into the synthesis, however precedent by Hoyveda ${ }^{147}$ indicates that this should be a relatively uncomplicated adaptation. Nevertheless, we are equally interested in the analogue without the ethyl substituent as this may provide valuable insight into the biological activity of pel A and potentially lead to the development of simplified analogues. Similarly, our strategy facilitates the synthesis of both the C-16 methylated, and unmethylated, analogues which again will be of interest during biological testing. 
There are however, a few improvements and changes that need be made to our strategy. Since the determination of pel A's absolute stereochemistry by De Brabander, ${ }^{204}$ the most pressing adaptation required is the synthesis of the correct enantiomer of the C-12 to C-24 fragment. In particular, this requires the synthesis of the $(S)$-enantiomer of the key intermediate B-hydroxyketone 24. Although precedent by Paterson ${ }^{164}$ could be followed for the synthesis of $\mathbf{2 4}$, it would be valuable to first investigate some alternative strategies including the BINOL-Ti catalysed Mukaiyama aldol reaction between the silyl enol ether of acetone and methacrolein, or acrolein..$^{93}$

A future objective of this research is also to find a more active RCM catalyst for the synthesis of the bis-silyl ether $\mathbf{6 5}$. Not only would this reduce the amount of catalyst required but would greatly facilitate the purification process.

However, more importantly, during the synthesis of the C-12 to C-24 fragment of pel $\mathrm{A}$ and subsequent aldol studies, valuable insight into the origin of 1,5-anti induction in boron-mediated aldol reactions has been gained. ${ }^{194}$ Our studies point to three modes of operation in effect during boron-mediated aldol reactions. First, in acyclic systems, the judicious use of electron-rich $\pi$-systems as $\beta$-hydroxy protecting groups must be used to provide for favourable $\pi$ stacking and hence high selectivities. Second, in some cyclic systems containing aromatic moieties, such as those studied during the synthesis of pel A, the rigidity of the system facilitates favourable $\pi$-stacking interactions, and hence, diastereoselectivies. And finally, in other cyclic systems, simple steric hindrance is responsible for the observed selectivities. These insights will undoubtedly aid in the planning of synthetic routes during the synthesis of further natural products. 


\section{Chapter9:}

\section{Experimental Section}

\subsection{General:}

Unless otherwise stated, the following conditions apply. All reactions were performed under argon in oven-dried glassware using dry solvents and standard syringe techniques. Diethyl ether and THF were distilled from sodium benzophenone ketyl radical ion. Dichloromethane, triethylamine and methanol were distilled from calcium hydride. Diisopropylamine and pyridine were distilled from sodium hydroxide. Benzene was successively shaken with concentrated sulfuric acid, water, dilute sodium hydroxide and water, before being dried with calcium hydride and distilled. Acetone was distilled from potassium carbonate. Anhydrous dimethylformamide was purchased from Aldrich Chemical Company and used without further purification. All other reagents were of commercial quality and distilled prior to use if necessary.

Reaction progress was monitored using aluminium backed TLC plates pre-coated with silica UV254 and visualised by either UV radiation $(254 \mathrm{~nm})$, ceric ammonium molybdate dip or using an iodine tank unless otherwise stated. Purification of products via flash chromatography was conducted using a column filled with silica gel 60 (220240 mesh) with the solvent systems as indicted. ${ }^{1} \mathrm{H}$ and ${ }^{13} \mathrm{C}$ NMR spectra were recorded in $\mathrm{CDCl}_{3}$ on a Varian Inova at 300 and $75 \mathrm{MHz}$ respectively; and are referenced to solvent peaks $\left({ }^{1} \mathrm{H}\right.$ - residual $\left.\mathrm{CHCl}_{3}(7.26 \mathrm{ppm}),{ }^{13} \mathrm{C}-\mathrm{CDCl}_{3},(77.0 \mathrm{ppm})\right)$. Infrared spectra were obtained on a Biorad FTS-7 spectrometer or a Bruker Tensor 27 FTIR spectrometer. Electrospray High-resolution mass spectroscopy was recorded on a Mariner time of flight spectrometer. Diastereoselectivities were determined by averaging the ${ }^{13} \mathrm{C}$ NMR peak heights for the diastereotopic signals of the crude product. 
Experimental data is reported in chronological order. The following compounds were synthesised according to literature precedent: 3-(tert-butyldimethylsiloxy)propanol (32) ${ }^{98}$ Des Martin Periodinane, ${ }^{99}(S)$-4-benzyl-3-butyryl-2-oxazolidinone $(37)^{195},(+)$ - $(4 S)$ 3-[(2'R)-2'-((tert-butyldimethylsiloxy)-methyl)-butanoyl]-4-benzyl-2-oxazolidinone $(38),{ }^{79}(-)-(2 S)-2-\left(\left(\right.\right.$ tert-butyldimethyl-siloxy)methyl)butanol (39),${ }^{79} \quad(-)-(2 S)-2-(($ tertbutyldimethylsiloxy)methyl)-butanal (3c) ${ }^{79}$ isopropyltriphenylphosphonium bromide, ${ }^{108}$ dihydro-2-(tosyloxy)-3,3-dimethyl-(2H)-furanone (17b), ${ }^{113}$ 4-hydroxy-5-hexene-2-one (24a), ${ }^{137}$ Grubbs second generation ruthenium catalyst (62), ${ }^{141}$ benzyltrichloroacetimidate (79) $)^{134, X X I V}$, dicyclohexylboron hydride $\left(\mathrm{Cy}_{2} \mathrm{BH}\right),{ }^{196}$ dicyclohexylborontriflate $\left(\mathrm{Cy}_{2} \mathrm{BOTf}\right)^{197}$, dicyclohexylboronchloride $\left(\mathrm{Cy}_{2} \mathrm{BCl}\right),{ }^{197}$ diisopinocampheylboron hydride $\left(\mathrm{Ipc}_{2} \mathrm{BH}\right)^{198}$, diisopinocamphenylboron chloride $\left(\mathrm{Ipc}_{2} \mathrm{BCl}\right)^{199},(R)$-4-hydroxyl-5-methylhexan-2-one (94a), ${ }^{176 a, 176 b}(R)$-4-hydroxy-5,5-dimethyl-hexan-2-one (94b), ${ }^{176 a, 176 b \quad 4-}$ methoxy-3-butene-2-one (104), ${ }^{200}$ 3-butene-4-(hepta-1,6-diene-4-oxy)-2-one (107) ${ }^{178}$ and 1-(6-allyl-4-hydroxy)tetrahydropyran-2-yl)-propan-2-one (108). ${ }^{178}$

XXIV The purification procedure reported in the literature for the synthesis of benzyl trichloroacetimidate, involving the washing the initially formed filtrate with pentane, proved to be unsuitable as two immiscible layers were formed. Consequently, the residual oil was purified by rapid distillation at $0.1 \mathrm{~mm} / \mathrm{Hg}$. It is imperative that this distillation is performed rapidly as the intramolecular thermal rearrangement of benzyl trichloroacetimidate to the $\mathrm{N}$-benzyl amide is frequently observed after prolonged heating of the substrate. 


\subsection{Experimental for Chapter 2:}

3-(tert-Butyldimethylsiloxy)propanal (33). To a stirred solution of 3-(tertbutyldimethylsiloxy)propanol (32) $(2.20 \mathrm{~g}, 11.55 \mathrm{mmol})$ in $\mathrm{CH}_{2} \mathrm{Cl}_{2}(80 \mathrm{~mL})$ was added water $(200 \mu \mathrm{L})$ followed Dess-Martin periodinane $(6.30 \mathrm{~g}, 13.86 \mathrm{mmol})$. After $4 \mathrm{~h}$ at room temperature additional Dess-Martin periodinane $(6.30 \mathrm{~g}, 13.86 \mathrm{mmol})$ was added and the reaction stirred at room temperature overnight. The solution was then diluted with $100 \mathrm{~mL}$ of $\mathrm{Et}_{2} \mathrm{O}$ and treated with a 1:1 mixture of saturated $\mathrm{NaHCO}_{3}: \mathrm{NaS}_{2} \mathrm{O}_{3}(100 \mathrm{~mL})$ for 15 minutes. The ether layer was collected and the aqueous phase extracted once with ether. The combined organic layers were then washed with brine, dried over $\mathrm{MgSO}_{4}$ and the solvent carefully removed under reduced pressure. The residual oil was then purified by flash chromatography (10:1 pentane: $\left.\mathrm{Et}_{2} \mathrm{O}\right)$ to give 3-(tert-butyldimethylsiloxy)propanal (33) $(1.09 \mathrm{~g}, 50 \%)$. NMR spectral data matched that previously reported. ${ }^{201}$

2-(Hydroxymethyl)butan-1-ol (35). This was synthesised in the manner previously reported ${ }^{100}$ with the exception that the solution was refluxed in $\mathrm{Et}_{2} \mathrm{O}$ for $2.5 \mathrm{~h}$ and the product purified by bulb-to-bulb distillation.

2-((tert-Butyldimethyl-siloxy)methyl)butanol (36). To solid $\mathrm{NaH}$ (204 mg, 8.49 mmol), washed three times with hexanes, was added dropwise a solution of 2-ethyl-1,3propanediol (35) (590 mg, $5.66 \mathrm{mmol})$ in THF $(13 \mathrm{~mL})$. The reaction was stirred vigorously for $45 \mathrm{~min}$ at room temperature. Tert-butyldimethylsilylchloride $(854 \mathrm{mg}$, $5.66 \mathrm{mmol})$ in THF ( $4 \mathrm{~mL})$ was then added in one portion and the reaction stirred vigorously for 45 minutes before being quenched cautiously with water. The resulting solution was extracted with $\mathrm{Et}_{2} \mathrm{O}$, washed with $10 \% \mathrm{~K}_{2} \mathrm{CO}_{3}$, brine and dried over $\mathrm{Na}_{2} \mathrm{SO}_{4}$. After removal of the solvent under reduced pressure, the residual oil was purified by flash chromatography $(10: 1$ hexanes:EtOAc), to give $36(1.11 \mathrm{~g}, 90 \%)$ as a colourless oil. NMR spectral data matched that previously reported. ${ }^{100}$ 
2-(tert-Butyldimethylsiloxymethyl)butanal (3b). To a stirred solution of 2-(tertbutyldimethyl-siloxymethyl) butanol (37) (395 mg, $1.81 \mathrm{mmol}$ ) in $\mathrm{CH}_{2} \mathrm{Cl}_{2}(12.4 \mathrm{~mL}$ ) was added Dess-Martin periodinane $(1.15 \mathrm{~g}, 2.71 \mathrm{mmol})$ and the reaction stirred for $6 \mathrm{~h}$ at room temperature. ${ }^{1} \mathrm{H}$ NMR analysis of a small portion of the reaction product indicated that some of the starting substrate 37 was still present and a further $1.15 \mathrm{~g}$ of the periodinane was added and the reaction stirred at room temperature overnight. The solution was diluted further with $\mathrm{CH}_{2} \mathrm{Cl}_{2}(50 \mathrm{~mL})$ and filtered through a small, $0.5 \mathrm{~cm}$ thick, plug of silica gel. The residual oil was purified by flash chromatography (10:1 pentane: $\left.\mathrm{Et}_{2} \mathrm{O}\right)$ to give $\mathbf{3} \mathbf{b}(170 \mathrm{mg}, 44 \%)$. NMR spectral data matched that previously reported. ${ }^{79}$

2-(Acetoxymethyl)butanol (43b) and 2-(Acetoxymethyl)but-1-ylacetate (42). To a rapidly stirred solution of 2-(hydroxymethyl)butan-1-ol (35) in pyridine $(20 \mathrm{~mL})$ at $0{ }^{\circ} \mathrm{C}$ was added dropwise acetyl chloride $(942 \mu \mathrm{L}, 13.25 \mathrm{mmol})$. The reaction was allowed to warm to room temperature overnight before being quenched with water and extracted twice with EtOAc. The organic extracts were washed once with satd. aq. $\mathrm{NaHCO}_{3}$ solution, brine and dried over $\mathrm{Na}_{2} \mathrm{SO}_{4}$. The residual pyridine was removed by codistillation with toluene and the crude material purified by gradient flash chromatography (5:1 to 3:1 hexanes:EtOAc) to give $43 \mathrm{~b}(637 \mathrm{mg}, 40 \%)$ and $42(821 \mathrm{mg}, 40 \%)$. NMR spectral data matched those previously reported. ${ }^{100}$

2-(Acetoxymethyl)butanal (44b). This compound was synthesised using a variety of conditions, as indicated below. In all instances, the NMR spectral data matched that previously reported. ${ }^{100}$

a) Small scale Des-Martin oxidation of 2-(acetoxymethyl)butanol (43b). To a stirred solution of $43 \mathrm{~b}(8 \mathrm{mg}, 0.055 \mathrm{mmol})$ in $\mathrm{CH}_{2} \mathrm{Cl}_{2}(500 \mu \mathrm{L})$ was added Dess-Martin periodinane ( $35 \mathrm{mg}, 0.082 \mathrm{mmol}$ ). The solution was stirred for $2.5 \mathrm{~h}$ at room temperature then an additional $35 \mathrm{mg}$ of Dess-Martin periodinane was added. After an additional $3 \mathrm{~h}$ the resulting solution was loaded onto a Pasteur pipette sized silica gel column and eluted with $\mathrm{CH}_{2} \mathrm{Cl}_{2}(2 \mathrm{~mL})$ to give $44 \mathbf{b}$ as a colourless oil. 
b) Modified Swern oxidation of $\mathbf{4 3 b}$. To a solution of oxalyl chloride ( $773 \mu \mathrm{L}, 8.87$ $\mathrm{mmol})$ in $\mathrm{CH}_{2} \mathrm{Cl}_{2}(72 \mathrm{~mL})$ at $-78^{\circ} \mathrm{C}$, was added dropwise DMSO $(1.26 \mu \mathrm{L}, 17.73 \mathrm{mmol})$. The solution was stirred for 15 minutes before a solution of $\mathbf{4 3 b}(1.08 \mathrm{~g}, 7.39 \mathrm{mmol})$ in $\mathrm{CH}_{2} \mathrm{Cl}_{2}(21 \mathrm{~mL})$ was added dropwise. The resulting solution was stirred at $-78^{\circ} \mathrm{C}$ for 30 minutes, $\mathrm{Et}_{3} \mathrm{~N}(4.02 \mathrm{~mL}, 28.8 \mathrm{mmol})$ was slowly added, at which point the cloudy solution turned clear. The solution was stirred for an additional $1.25 \mathrm{~h}$ at $-78^{\circ} \mathrm{C}$ then at 0 ${ }^{\circ} \mathrm{C}$ for 5 minutes before being diluted with sat. aq. $\mathrm{NaHCO}_{3}$. The resulting solution was then extracted twice with $\mathrm{CH}_{2} \mathrm{Cl}_{2}$, and the organic extracts washed twice with a $1 \mathrm{M}$ sodium bisulfite solution, once with brine and dried over $\mathrm{MgSO}_{4}$. The solvent was removed under reduced pressure and the residual oil diluted with $\mathrm{CH}_{2} \mathrm{Cl}_{2}(20 \mathrm{~mL})$ and purified immediately by passage through a small, $1.5 \mathrm{~cm}$ thick, $3 \mathrm{~cm}$ diameter, silica gel plug. The silica plug was then washed with a further $\mathrm{CH}_{2} \mathrm{Cl}_{2},(20 \mathrm{~mL})$ and the solvent removed under reduced pressure to give $44 \mathrm{~b}(672 \mathrm{mg}, 63 \%)$ that was used without further purification.

c) From the periodate oxidative cleavage of 4-(acetoxymethyl)-2-methyl-hex-2-ene (46). 4-(acetoxymethyl)-2-methyl-hex-2-ene (46) (6 mg) was dissolved in THF (540 $\mu \mathrm{L}) \mathrm{H}_{2} \mathrm{O}$ (300 $\mu \mathrm{L}$ ). A $70 \mu \mathrm{L}$ aliquot of this solution, which equates to $0.5 \mathrm{mg}$ or $2.94 \mu \mathrm{mol}$ of 9 , was then transferred to a second flask and $3 \mu \mathrm{L}(0.3 \mu \mathrm{mol})$ of a 2.5 wt \% solution of osmium tetraoxide in tert-butanol added. The solution was stirred at room temperature for 10 minutes before $\mathrm{NaIO}_{4}(2.5 \mathrm{mg}, 11.5 \mu \mathrm{mol})$ was added. The solution was stirred vigorously at room temperature, and the progress of the reaction monitored by GC using a $60 \mathrm{~m} \times 0.25 \mathrm{~mm}$ Cyclodex-B ${ }^{\mathrm{TM}}$ column and the following conditions: Inlet: Split $275^{\circ} \mathrm{C}$; Detector: FID $320^{\circ} \mathrm{C}$; Carrier gas: hydrogen; Flow: $35.8 \mathrm{~cm}^{3} \mathrm{~s}^{-1}$; Temperature programme: Isothermal at $60^{\circ} \mathrm{C}$. The GC conditions were as follows. After $2 \mathrm{~h}$ no starting material was present and two peaks with retention times of 116.5 and 119.5 minutes were observed on the GC trace.

This experiment was previously conducted using $15 \mathrm{mg}$ of $\mathbf{4 6}$. After 3 hours the solution was diluted with $\mathrm{Et}_{2} \mathrm{O},(20 \mathrm{~mL})$, washed with brine, dried over $\mathrm{MgSO}_{4}$ and filtered. The 
solvent was then removed under reduced pressure to give $\mathbf{4 4 b}$ as a colourless oil. NMR spectral data matched that previously reported. ${ }^{100}$

4-(Acetoxymethyl)-2-methyl-hex-2-ene (47). To a mixture of isopropyltriphenylphosphonium bromide $(720 \mathrm{mg}, 1.87 \mathrm{mmol})$ in THF $(20 \mathrm{~mL})$ at $0{ }^{\circ} \mathrm{C}$ was added a $1.6 \mathrm{M}$ solution of $n$-BuLi in hexanes $(1.17 \mathrm{~mL}, 1.87 \mathrm{mmol})$. A red solution was observed due to the formation of triphenylphosphoranylidene isopropane. After the mixture was stirred for $1 \mathrm{~h}$ at room temperature, a solution of $44 \mathrm{~b}(245 \mathrm{mg}, 1.70 \mathrm{mmol})$ in dry THF $(1.5 \mathrm{~mL})$ was added dropwise. The resulting mixture was stirred at room temperature for $4 \mathrm{~h}$ then poured into $50 \mathrm{~mL}$ of hexanes. The precipitated triphenylphosphine oxide was removed by filtration and the resulting solution concentrated in vacuo. The residue was purified by gradient flash chromatography (hexane, 50:1 hexanes:EtOAc) to give 47 (120 mg, 42\%) as a colourless oil. ${ }^{1} \mathrm{H}$ NMR: $\delta 4.85(\mathrm{~d}, J=9.9 \mathrm{~Hz}, 1 \mathrm{H}), 3.92(\mathrm{~d}, J=6.9 \mathrm{~Hz}, 2 \mathrm{H}), 2.51(\mathrm{~m}$, $1 \mathrm{H}), 2.06(\mathrm{~s}, 3 \mathrm{H}), 1.74(\mathrm{~s}, 3 \mathrm{H}), 1.65(\mathrm{~s}, 3 \mathrm{H}), 1.51(\mathrm{~m}, 1 \mathrm{H}), 1.21(\mathrm{~m}, 1 \mathrm{H}), 0.87(\mathrm{t}, J=7.5$ $\mathrm{Hz}, 3 \mathrm{H}) \mathrm{ppm} .{ }^{13} \mathrm{C}$ NMR: $\delta 171.5,134.4,125.3,68.0,39.3,26.1,25.2,21.3,18.5,11.7$ ppm. IR (neat): 2966, 1739, 1230, 1035, $733 \mathrm{~cm}^{-1}$. 


\section{3: Experimental for Chapter 3:}

Dihydro-2-(allyloxy)-4,4-dimethyl-(2H)-furanone (17c). To a solution of pantolactone (17) $(10.0 \mathrm{~g}, 76.8 \mathrm{mmol})$ in $\mathrm{DMF}(80 \mathrm{~mL})$ at $0{ }^{\circ} \mathrm{C}$ was added a $1 \mathrm{M}$ solution of potassium tert-butoxide in THF $(76.8 \mathrm{~mL}, 76.8 \mathrm{mmol})$. The cooling bath was then removed and the solution stirred at room temperature for 30 minutes. A solution of allyl bromide (6.99 $\mathrm{mL}, 80.6 \mathrm{mmol})$ in THF $(7 \mathrm{~mL})$ was then added dropwise and the resulting mixture stirred at room temperature overnight before being quenched with water and extracted twice with $\mathrm{Et}_{2} \mathrm{O}$. The combined organic extracts were washed twice with sat. aq. $\mathrm{NaHCO}_{3}$, once with brine, dried over $\mathrm{MgSO}_{4}$ and the solvent removed under reduced pressure. Flash chromatography of the crude product (5:1 hexanes:EtOAc) gave 17c $(10.6$ g, $81 \%$ ) as a colourless oil. ${ }^{1} \mathrm{H}$ NMR: $\delta 5.90(\mathrm{~m}, 1 \mathrm{H}), 5.32(\mathrm{dd}, J=17.1,1.2 \mathrm{~Hz}, 1 \mathrm{H}), 5.24$ $(\mathrm{d}, J=10.2 \mathrm{~Hz}, 1 \mathrm{H}), 4.46(\mathrm{dd}, J=12.9,5.1 \mathrm{~Hz}, 1 \mathrm{H}), 4.20(\mathrm{dd}, J=12.9,6.3 \mathrm{~Hz}, 1 \mathrm{H})$, $3.99(\mathrm{~d}, J=8.7 \mathrm{~Hz}, 1 \mathrm{H}), 3.89(\mathrm{~d}, J=8.7 \mathrm{~Hz}, 1 \mathrm{H}), 3.74(\mathrm{~s}, 1 \mathrm{H}), 1.17(\mathrm{~s}, 3 \mathrm{H}), 1.10(\mathrm{~s}, 3 \mathrm{H})$ ppm. ${ }^{13} \mathrm{C}$ NMR: $\delta 175.3,133.8,118.0,80.7,76.3,71.6,40.3,23.3,19.2$ ppm. IR (neat): 2971, 1788, $1124 \mathrm{~cm}^{-1}$. HRMS: Calcd for $\mathrm{C}_{9} \mathrm{H}_{14} \mathrm{O}_{3}\left(\mathrm{M}+\mathrm{H}^{+}\right)$: 171.1016. Found: 171.1020.

2,2-Dimethyl-3-(3-propeneoxy)butane-1-4-diol (53). A $25 \mathrm{~mL}$ flask equipped with side arm and reflux condensor was charged with $\mathrm{LiAlH}_{4}(305 \mathrm{mg}, 7.85 \mathrm{mmol})$ and THF (10 $\mathrm{mL})$. A solution of $\mathbf{1 7} \mathbf{c}(891 \mathrm{mg}, 5.23 \mathrm{mmol})$ in THF $(5 \mathrm{~mL})$ was then added cautiously dropwise over 15 minutes. The resulting solution was refluxed for $3 \mathrm{~h}$ before being cooled to $0{ }^{\circ} \mathrm{C}$ and quenched by the portion-wise addition of hydrated sodium sulfate. The residue was diluted with ethanol $(50 \mathrm{~mL})$, filtered though a $0.5 \mathrm{~cm} \times 3 \mathrm{~cm}$ diameter silica gel plug, dried with anhydrous $\mathrm{Na}_{2} \mathrm{SO}_{4}$, and the solvents removed under reduced pressure. Flash chromatography of the crude product (1:1 hexanes:EtOAc) gave 53 (690 $\mathrm{mg}, 76 \%$ ) as a colourless oil. ${ }^{1} \mathrm{H}$ NMR: $\delta 5.95(\mathrm{~m}, 1 \mathrm{H}), 5.29(\mathrm{dd}, J=17.1,1.5 \mathrm{~Hz}, 1 \mathrm{H})$, 5.19 (dd, $J=10.5,1.2 \mathrm{~Hz}, 1 \mathrm{H}$ ), 4.19 (ddt, $J=12.6,5.4,1.5 \mathrm{~Hz}, 1 \mathrm{H}$ ), 4.07 (ddt, $J=12.6$, $5.4,1.5 \mathrm{~Hz}, 1 \mathrm{H}), 3.80(\mathrm{dd}, J=12.0,3.90 \mathrm{~Hz}, 1 \mathrm{H}), 3.70(\mathrm{dd}, J=12.0,4.2 \mathrm{~Hz}, 1 \mathrm{H}), 3.20$ (dd, $J=4.2,3.9 \mathrm{~Hz}, 1 \mathrm{H}), 2.36(\mathrm{bs}, 2 \mathrm{H}), 0.96(\mathrm{~s}, 3 \mathrm{H}), 0.94(\mathrm{~s}, 3 \mathrm{H}) \mathrm{ppm} .{ }^{13} \mathrm{C}$ NMR: $\delta$ 
134.7, 117.1, 85.7, 72.6, 69.1, 60.6, 39.1, 23.0, 21.3 ppm. IR (neat): 3345, 2967, 2876, $1048 \mathrm{~cm}^{-1}$. HRMS: Calcd for $\mathrm{C}_{9} \mathrm{H}_{18} \mathrm{O}_{3} 175.1334\left(\mathrm{M}+\mathrm{H}^{+}\right)$. Found 175.1329.

1,4-Dibenzyloxy-2,2-dimethyl-3-(3-propeneoxy)-butane (54). To a $25 \mathrm{~mL}$ flask containing $\mathrm{NaH}$ (491 mg, $20.5 \mathrm{mmol}$ ) (washed three times with hexanes) in DMF (6.5 $\mathrm{mL})$ at $0{ }^{\circ} \mathrm{C}$ was added a solution of $\mathbf{5 3}(713 \mathrm{mg}, 4.09 \mathrm{mmol})$ in DMF (5 mL) over five minutes. When gas evolution had ceased, benzyl bromide (998 $\mu \mathrm{l}, 8.39 \mathrm{mmol})$ was added dropwise and the reaction mixture allowed to warm to room temperature overnight. The solution was then cautiously quenched with water, extracted twice with $\mathrm{Et}_{2} \mathrm{O}$ and the combined organic extracts washed twice with sat. aq. $\mathrm{NaCO}_{3}$ solution, once with brine, dried over $\mathrm{MgSO}_{4}$ and the solvent removed under reduced pressure. Flash chromatography of the crude product (hexanes) gave the benzylated ether $54(1.30 \mathrm{~g}$, $90 \%)$ as a colourless oil. ${ }^{1} \mathrm{H}$ NMR: $\delta 7.32(\mathrm{~m}, 10 \mathrm{H}), 5.93(\mathrm{~m}, 1 \mathrm{H}), 5.24(\mathrm{dd}, J=17.1,1.8$ $\mathrm{Hz}, 1 \mathrm{H}$ ), 5.10 (dd, $J=10.2,1.5 \mathrm{~Hz}, 1 \mathrm{H}), 4.52$ (s, 2H), 4.46 (s, 2H), 4.34 (ddt, $J=12.6$, $5.4,1.5 \mathrm{~Hz}, 1 \mathrm{H}), 4.03$ (ddt, $J=12.6,5.4,1.5 \mathrm{~Hz}, 1 \mathrm{H}), 3.58(\mathrm{~m}, 2 \mathrm{H}), 3.38$ (d, $J=8.7 \mathrm{~Hz}$, $1 \mathrm{H}), 3.15$ (d, $J=8.7 \mathrm{~Hz}, 1 \mathrm{H}), 0.95$ (s, 3H) 0.92, (s, 3H) ppm. ${ }^{13} \mathrm{C}$ NMR: $\delta 138.9,138.6$, $135.8,128.3,127.8,127.7,127.4,115.7,82.5,77.2,73.2,72.0,38.7,20.9,22.2$ ppm. IR (neat): $3028,2906,2859,1092 \mathrm{~cm}^{-1}$.

1,4-Dibenzyloxy-2,2-dimethyl-3-hydroxy-butane (55). p-Toluenesulfinic acid (82 mg, $0.52 \mathrm{mmol}$ ) was added at room temperature to a solution of 54 (167 $\mathrm{mg}, 0.47 \mathrm{mmol})$ and $\mathrm{Pd}\left(\mathrm{PPh}_{3}\right)_{4}(33 \mathrm{mg}, 0.03 \mathrm{mmol})$ in THF $(4.7 \mathrm{~mL})$. The reaction mixture was stirred at room temperature for $7 \mathrm{~h}$ before $\mathrm{Et}_{3} \mathrm{~N}(13 \mu \mathrm{l})$ was added. The solvent was then removed under reduced pressure and the residue purified immediately by gradient flash chromatography (50:1 to 20:1 hexanes:EtOAc) to give $5(122 \mathrm{mg}, 83 \%)$ as a colourless oil. ${ }^{1} \mathrm{H}$ NMR: $\delta$ $7.37(\mathrm{~m}, 10 \mathrm{H}), 4.57(\mathrm{~s}, 2 \mathrm{H}), 4.50(\mathrm{~s}, 2 \mathrm{H}), 3.78(\mathrm{ddd}, J=8.4,3.3,3.0 \mathrm{~Hz}, 1 \mathrm{H}), 3.64(\mathrm{dd}, J$ $=9.6,3.0 \mathrm{~Hz}, 1 \mathrm{H}), 3.49(\mathrm{dd}, J=9.6,8.4 \mathrm{~Hz}, 1 \mathrm{H}), 3.38(\mathrm{~d}, J=8.7 \mathrm{~Hz}, 1 \mathrm{H}), 3.27(\mathrm{~d}, J=$ $8.7 \mathrm{~Hz}, 1 \mathrm{H}), 3.03(\mathrm{~d}, J=3.3 \mathrm{~Hz}, 1 \mathrm{H}), 0.97$ (s, 3H), 0.96 (s, 3H) ppm. ${ }^{13} \mathrm{C}$ NMR: $\delta$ 138.4, $129.5,128.2,127.8,127.6,127.5,78.2,75.7,73.4,73.3,71.7,37.4,21.6,20.3$ ppm. IR 
(neat): $3498,2987,2848,1105 \mathrm{~cm}^{-1}$. HRMS: Calcd for $\mathrm{C}_{20} \mathrm{H}_{26} \mathrm{O}_{3} 315.1950\left(\mathrm{M}+\mathrm{H}^{+}\right)$. Found 315.1955 .

1,4-Dibenzyloxy-3,3-dimethyl-2-butanone (8). To a solution of 54 (100 $\mathrm{mg}, 0.32 \mathrm{mmol})$ in $\mathrm{CH}_{2} \mathrm{Cl}_{2}(1.6 \mathrm{~mL})$ was added pyridinium dichromate $(181 \mathrm{mg}, 0.48 \mathrm{mmol})$, freshly activated $3 \AA$ molecular sieve powder $(260 \mathrm{mg})$ then anhydrous $\mathrm{AcOH}(32 \mu \mathrm{l}, 0.55$ $\mathrm{mmol})$. The reaction was stirred at room temperature for 15 minutes before being diluted with $\mathrm{CH}_{2} \mathrm{Cl}_{2}$ and filtered though a small $(0.5 \mathrm{~cm})$ bilayer Celite ${ }^{\mathrm{TM}} /$ silica gel plug. The filtrate was washed with water then brine and the organic phase dried over $\mathrm{MgSO}_{4}$. The solvent was removed under reduced pressure and residue purified via flash chromatography (50:1 hexanes:EtOAc) to give $8(81 \mathrm{mg}, 82 \%)$ as a colourless oil. ${ }^{1} \mathrm{H}$ NMR: $\delta 7.35$ (m, 10H), 4.58 (s, 2H), 4.48 (s, 2H), 4.38 (s, 2H), 3.44 (s, 2H), 1.17 (s, 6H) ppm. ${ }^{13} \mathrm{C}$ NMR: $\delta$ 210.8, 138.2, 137.8, 128.7, 128.6, 128.2, 128.1, 127.9, 127.8, 77.3, 73.6, 73.3, 72.4, 47.6, 22.1 ppm. IR (neat): 3031, 2967, 2932, 2869, 1723, $1100 \mathrm{~cm}^{-1}$. HRMS: Calcd for $\mathrm{C}_{20} \mathrm{H}_{24} \mathrm{O}_{3} 313.1805\left(\mathrm{M}+\mathrm{H}^{+}\right)$. Found 313.1798. 


\subsection{Experimental for Chapter 4:}

4-Hydroxy-5-methylhex-5-en-2-one (24b). To a solution of diisopropylamine (1.37 mL, $9.81 \mathrm{mmol})$ in dry THF $(40 \mathrm{~mL})$ cooled to $-78^{\circ} \mathrm{C}$ was added a $2 \mathrm{M}$ solution of $n$-BuLi in hexanes $(4.90 \mathrm{~mL}, 9.81 \mathrm{mmol})$. After stirring at $-78^{\circ} \mathrm{C}$ for $10 \mathrm{~min}$, a solution of acetone $(600 \mu \mathrm{l}, 8.17 \mathrm{mmol})$ in $\mathrm{THF}(4 \mathrm{~mL})$ was added slowly over $5 \mathrm{~min}$. Stirring at $-78^{\circ} \mathrm{C}$ was continued for $50 \mathrm{~min}$ and then a solution of methacrolein $(811 \mu \mathrm{l}, 9.81 \mathrm{mmol})$ in THF (4 $\mathrm{mL}$ ) added. The mixture was stirred at $-78{ }^{\circ} \mathrm{C}$ for a further $10 \mathrm{~min}$, followed by the addition of sat. aq. $\mathrm{NH}_{4} \mathrm{Cl}$. The mixture was then extracted twice with $\mathrm{Et}_{2} \mathrm{O}$ and the organic extracts washed with brine, dried over $\mathrm{MgSO}_{4}$ and the solvent removed under reduced pressure. Bulb-to-bulb distillation $\left(58-60{ }^{\circ} \mathrm{C}, 0.4 \mathrm{~mm} / \mathrm{Hg}\right)$ gave $24 \mathbf{b}(882 \mathrm{mg}$, $84 \%$ ) as a colourless oil. NMR spectral data matched that previously reported. ${ }^{202}$

4-(3-Butenoyl)hex-5-en-2-one (57a). To a solution of the ß-hydroxy-ketone $24 a$ (1.00 g, $9.64 \mathrm{mmol})$ in $\mathrm{CH}_{2} \mathrm{Cl}_{2}(45 \mathrm{~mL})$ was added vinylacetic acid $(819 \mu \mathrm{L}, 9.64 \mathrm{mmol})$, dicyclohexylcarbodiimide (1.99 g, $9.64 \mathrm{mmol})$ and DMAP (54 mg, $0.45 \mathrm{mmol}$ ) and the reaction stirred at room temperature overnight. The precipitated dicyclohexyl urea was removed by filtration and the precipitate washed with $60 \mathrm{~mL}$ of ice-cold $\mathrm{CH}_{2} \mathrm{Cl}_{2}$. The combined organic extracts were then washed once with water, dried over $\mathrm{MgSO}_{4}$, filtered, and the solvent removed under reduced pressure. The crude material was initially purified by flash chromatography (5:1 hexanes:EtOAc) to give $\mathbf{5 7 a}$ containing small amounts of dicyclohexyl urea. This was further purified by bulb-to-bulb distillation $\left(90-100{ }^{\circ} \mathrm{C}, 0.4\right.$ $\mathrm{mm} / \mathrm{Hg})$ to give pure $57 \mathbf{a}(1.28 \mathrm{~g}, 80 \%)$ as a colourless oil. ${ }^{1} \mathrm{H}$ NMR: $\delta 5.89(\mathrm{~m}, 2 \mathrm{H}) 5.70$ (m, 1H), $5.26(\mathrm{~m}, 4 \mathrm{H}), 3.12(\mathrm{dd}, J=1.4 \mathrm{~Hz}, 1 \mathrm{H}), 3.10(\mathrm{dd}, J=1.4 \mathrm{~Hz}, 1 \mathrm{H}), 2.88(\mathrm{dd}, J=$ $16.5,7.7 \mathrm{~Hz}, 1 \mathrm{H}), 2.71(\mathrm{dd}, J=16.5,5.5 \mathrm{~Hz}, 1 \mathrm{H}), 2.20$ (s, 3H). ${ }^{13} \mathrm{C}$ NMR: $\delta 204.5,170.3$, 135.2, 130.0, 118.6, 117.1, 70.5, 47.7, 39.1, 30.4 ppm. IR (neat): 3087, 2934, 1738, 1723, $1167 \mathrm{~cm}^{-1}$. HRMS: Calcd for $\mathrm{C}_{10} \mathrm{H}_{18} \mathrm{NO}_{3} 200.1291\left(\mathrm{M}+\mathrm{NH}_{4}^{+}\right)$. Found 200.1281 . 
4-(3-Butenoyl)-5-methylhex-5-en-2-one (57b). To a solution of 4-hydroxy-5-methyl-5hexen-2-one (24b) $(824 \mathrm{mg}, 6.43 \mathrm{mmol})$ in $\mathrm{CH}_{2} \mathrm{Cl}_{2}(30 \mathrm{~mL})$ was added vinylacetic acid (601 $\mu \mathrm{L}, 7.07 \mathrm{mmol})$, dicyclohexylcarbodiimide (1.46 g, $7.07 \mathrm{mmol})$ and DMAP (40 mg, $0.32 \mathrm{mmol}$ ) and the reaction stirred at room temperature overnight. The precipitated dicyclohexyl urea was then removed by filtration, and the precipitate washed with $50 \mathrm{~mL}$ of ice-cold $\mathrm{CH}_{2} \mathrm{Cl}_{2}$. The combined organic extracts were then washed once with water, dried over $\mathrm{MgSO}_{4}$, filtered, and the solvent removed under reduced pressure. The crude material was initially purified by flash chromatography (5:1 hexanes:EtOAc) to give $\mathbf{5 7 b}$ containing small amounts of dicyclohexyl urea. Further purification by bulb-to-bulb distillation $\left(85-95^{\circ} \mathrm{C}, 0.4 \mathrm{~mm} / \mathrm{Hg}\right)$ gave pure $\mathbf{5 7 b}(1.06 \mathrm{~g}, 84 \%)$ as a colourless oil. ${ }^{1} \mathrm{H}$ NMR: $\delta 5.92(\mathrm{~m}, 1 \mathrm{H}), 5.64(\mathrm{dd}, J=8.8,4.5 \mathrm{~Hz}, 1 \mathrm{H}), 5.21(\mathrm{~m}, 1 \mathrm{H}), 5.17(\mathrm{~m}, 1 \mathrm{H}), 5.02(\mathrm{~d}$, $J=0.85 \mathrm{~Hz}, 1 \mathrm{H}), 4.94(\mathrm{t}, J=1.4 \mathrm{~Hz}, 1 \mathrm{H}), 3.12(\mathrm{t}, J=1.4 \mathrm{~Hz}, 1 \mathrm{H}), 3.10(\mathrm{t}, J=1.4 \mathrm{~Hz}$, 1H), 2.88 (dd, $J=16.3,8.8 \mathrm{~Hz}, 1 \mathrm{H}), 2.70$ (dd, $J=16.3,4.5 \mathrm{~Hz}, 1 \mathrm{H}), 2.21$ (s, 3H), 1.77 (s, 3H) ppm. ${ }^{13} \mathrm{C}$ NMR: $\delta$ 204.8, 170.2, 142.2, 130.0, 118.6, 113.0, 72.9, 46.5, 39.1, 30.3, 18.3 ppm. IR (neat): 1736, 1719, 1360, 1158, $1057 \mathrm{~cm}^{-1}$. HRMS: Calcd for $\mathrm{C}_{11} \mathrm{H}_{20} \mathrm{NO}_{3}$ 214.1441 $\left(\mathrm{M}+\mathrm{NH}_{4}^{+}\right)$. Found 214.1438.

5-Acetonyl- $\boldsymbol{\delta}$-lact-3-en-1-one (56a). To a side-armed flask equipped with a reflux condenser containing $\mathbf{5 7 a}(56 \mathrm{mg}, 0.31 \mathrm{mmol})$ was added $\mathrm{CH}_{2} \mathrm{Cl}_{2}(20 \mathrm{~mL})$ and tricyclohexylphosphine[1,3-bis(2,4,6-trimethylphenyl)-4,5-dihydroimidazol-2-ylidene[benzylidine]ruthenium (IV) dichloride (62) (3 mg, $3.4 \mu \mathrm{mol}$ ). The resulting maroon solution was refluxed for $2.5 \mathrm{~h}$ then the solvent was removed under reduced pressure. Gradient flash chromatography (5:1 to $2: 1$ hexanes:EtOAc) of the brown residue gave 56a (43 mg, 90\%) a colourless oil. ${ }^{1} \mathrm{H}$ NMR: $\delta 5.85(\mathrm{~s}, 2 \mathrm{H}), 5.43(\mathrm{~m}, 1 \mathrm{H}), 3.09(\mathrm{~m}, 2 \mathrm{H})$, 3.01 (dd, $J=17.2,6.4 \mathrm{~Hz}, 1 \mathrm{H}), 2.81$ (dd, $J=17.2,6.5 \mathrm{~Hz}, 1 \mathrm{H}), 2.22(\mathrm{~s}, 3 \mathrm{H}) \mathrm{ppm} .{ }^{13} \mathrm{C}$ NMR: $\delta 204.3,168.2,125.6,122.5,75.4,48.4,30.7,29.8$ ppm. IR (neat):1732, 1713, 1362, 1272, 1220, $1072 \mathrm{~cm}^{-1}$. HRMS: Calcd for $\mathrm{C}_{8} \mathrm{H}_{14} \mathrm{NO}_{3} 172.0973\left(\mathrm{M}+\mathrm{NH}_{4}^{+}\right)$. Found 172.0968 . 
5-Acetonyl-4-methyl- $\delta$-lact-3-en-1-one (56b). To a side-armed flask equipped with a reflux condensor containing $\mathbf{5 7 b}(250 \mathrm{mg}, 1.27 \mathrm{mmol})$ was added $\mathrm{CH}_{2} \mathrm{Cl}_{2}(100 \mathrm{~mL})$ and tricyclohexylphosphine[1,3-bis(2,4,6-trimethylphenyl)-4,5-dihydroimidazol-2-ylidene[benzylidine]ruthenium (IV) dichloride (62) $(11 \mathrm{mg}, 0.013 \mathrm{mmol})$. The resulting maroon solution was refluxed for $2.5 \mathrm{~h}$ then the solvent was removed under reduced pressure. Gradient flash chromatography $(5: 1$ to $2: 1$ hexanes:EtOAc) of the brown residue gave 57b $(182 \mathrm{mg}, 85 \%)$ as a colourless oil. ${ }^{1} \mathrm{H}$ NMR: $\delta 5.54(\mathrm{~m}, 1 \mathrm{H}), 5.22(\mathrm{~m}, 1 \mathrm{H}), 3.06(\mathrm{~m}$, $2 \mathrm{H}), 2.83(\mathrm{~m}, 2 \mathrm{H}), 2.22(\mathrm{~s}, 3 \mathrm{H}), 1.74(\mathrm{~s}, 3 \mathrm{H}) \mathrm{ppm} .{ }^{13} \mathrm{C}$ NMR: $\delta$ 204.8, 169.0, 131.9, $117.6,78.9,46.8,31.3,30.2,18.8$ ppm. IR (neat): 1730, 1715, 1355, 1214, 1154, 1063 $\mathrm{cm}^{-1}$. HRMS: Calcd for $\mathrm{C}_{9} \mathrm{H}_{16} \mathrm{NO}_{3} 186.1134\left(\mathrm{M}+\mathrm{NH}_{4}^{+}\right)$. Found 186.1125. 


\subsection{Experimental for Chapter 5:}

But-3-en-1-oxydiphenylsilyl chloride (68a). To a stirred mixture of diphenylsilyldichloride $(330 \mu \mathrm{l}, 1.57 \mathrm{mmol})$ and $\mathrm{Et}_{3} \mathrm{~N}(241 \mu \mathrm{l}, 1.73 \mathrm{mmol})$ in $\mathrm{CH}_{2} \mathrm{Cl}_{2}(6 \mathrm{~mL})$ at $0{ }^{\circ} \mathrm{C}$ was added a solution of 3-buten-1-ol (135 $\mu \mathrm{l}, 1.57 \mathrm{mmol})$ in $\mathrm{CH}_{2} \mathrm{Cl}_{2}(2 \mathrm{~mL})$ dropwise. The reaction mixture was stirred at room temperature before being heated at reflux for 36 h. The solution was then cooled to room temperature and concentrated under reduced pressure. The resultant oil was dissolved in a 1:1 vol:vol ether:pentane mixture $(15 \mathrm{~mL})$, suction filtered, and reconcentrated to afford an oil, which was used without further purification.

But-3-en-1-oxydiisopropylsilyl chloride (68b). To a stirred mixture of diisopropylsilyldichloride $(4 \mathrm{~mL}, 22.2 \mathrm{mmol})$ and $\mathrm{Et}_{3} \mathrm{~N}(3.4 \mathrm{~mL}, 24.4 \mathrm{mmol})$ in $\mathrm{CH}_{2} \mathrm{Cl}_{2} 85 \mathrm{~mL}$ at $0{ }^{\circ} \mathrm{C}$ was added a solution of 3-buten-1-ol (1.91 mL, $22.2 \mathrm{mmol})$ in $\mathrm{CH}_{2} \mathrm{Cl}_{2}(20 \mathrm{~mL})$ over 30 $\mathrm{min}$. The reaction mixture was stirred at room temperature for $6 \mathrm{~h}$ before being heated at reflux for $48 \mathrm{~h}$. After cooling to room temperature and concentrating under reduced pressure, the resultant oil was dissolved in a 1:1 vol:vol ether:pentane mixture $(100 \mathrm{~mL})$, suction filtered and reconcentrated to afford an oil. Fractional distillation of the crude material gave $68 \mathrm{~b}$ as a colourless oil (bp $58-60^{\circ} \mathrm{C}, 1 \mathrm{~mm} / \mathrm{Hg}, 2.64 \mathrm{~g}, 54 \%$ ). ${ }^{1} \mathrm{H}$ NMR: $\delta$ $5.82(\mathrm{~m}, 1 \mathrm{H}), 5.06(\mathrm{~m}, 2 \mathrm{H}), 3.83(\mathrm{t}, J=6.6 \mathrm{~Hz}, 2 \mathrm{H}), 2.32(\mathrm{dt}, J=13.5,6.9 \mathrm{~Hz}, 2 \mathrm{H}) 1.07$ (m, 14H) ppm. ${ }^{13} \mathrm{C}$ NMR: $\delta 135.1,117.0,63.6,37.0,17.0,16.9,15.2$ ppm. IR (neat): $2954,2876,1109,994,922,886 \mathrm{~cm}^{-1}$.

But-3-en-1-oxydimethylsilyl chloride (68c). To a stirred mixture of dimethylsilyldichloride $(4.54 \mathrm{~mL}, 37.5 \mathrm{mmol})$ and $\mathrm{Et}_{3} \mathrm{~N}(5.75 \mathrm{~mL}, 41.25 \mathrm{mmol})$ in $\mathrm{CH}_{2} \mathrm{Cl}_{2}(120 \mathrm{~mL})$ at $0{ }^{\circ} \mathrm{C}$ was added a solution of 3-buten-1-ol $(3.23 \mathrm{~mL}, 37.5 \mathrm{mmol})$ in $\mathrm{CH}_{2} \mathrm{Cl}_{2}(45 \mathrm{~mL})$ ropwise over $45 \mathrm{~min}$. The reaction mixture was then stirred at room temperature for $16 \mathrm{~h}$ before being heated at reflux for $12 \mathrm{~h}$. After cooling to room 
temperature and concentrating under reduced pressure, the resultant oil was dissolved in a 1:1 vol:vol ether:pentane mixture $(120 \mathrm{~mL})$, suction filtered and reconcentrated to afford an oil. Fractional distillation of the crude material gave $1.06 \mathrm{~g}(17 \%)$ of $68 \mathrm{c}$ as a colourless oil (bp $\left.63-65^{\circ} \mathrm{C}, 7 \mathrm{~mm} / \mathrm{Hg}\right) .{ }^{1} \mathrm{H}$ NMR: $\delta 5.82(\mathrm{~m}, 1 \mathrm{H}), 5.10(\mathrm{~m}, 1 \mathrm{H}), 3.81(\mathrm{t}, J$ $=6.9 \mathrm{~Hz}, 2 \mathrm{H}), 2.36(\mathrm{dt}, J=13.5,6.9 \mathrm{~Hz}, 2 \mathrm{H}), 0.49(\mathrm{~s}, 6 \mathrm{H}) \mathrm{ppm}^{\mathrm{xxv}}$

\section{General procedure for the formation of Diphenylsilyl derivatives (66a) and (66b)}

To a $0{ }^{\circ} \mathrm{C}$ stirred solution of the crude but-3-enoxydiphenylsilylchloride (68a) $(2.5 \mathrm{mmol})$ and the appropriate $\beta$-hydroxyketone $(1.57 \mathrm{mmol})$ in $\mathrm{CH}_{2} \mathrm{Cl}_{2}(5 \mathrm{~mL})$ was added $\mathrm{Et}_{3} \mathrm{~N}(218$ $\mu 1,1.57 \mathrm{mmol}$ ) dropwise. The ice bath was then removed and the solution stirred at room temparature for $2.5 \mathrm{~h}$ before being quenched with satd. aq. $\mathrm{NaHCO}_{3}$. The solution was then extracted twice with $\mathrm{Et}_{2} \mathrm{O}$ and the organic extracts washed with water, brine, and dried over $\mathrm{MgSO}_{4}$. Filtration and concentration gave a residual oil, which was purified by flash chromatography on silica gel (20:1 hexanes:EtOAc) to give the diphenyl bissilylether as a colourless oil. Yields are reported for the total conversion from dichlorodiphenylsilylchloride (68a).

\section{4-(But-3-en-1-oxydiphenylsilyloxy)-5-methylhex-5-en-2-one (66a).}

Dichlorodiphenylsilane (68a) $(330 \mu \mathrm{l}, 1.57 \mathrm{mmol})$ gave $66 \mathbf{a}(335 \mathrm{mg}, 0.95 \mathrm{mmol})$ in an overall yield of $61 \%$. ${ }^{1} \mathrm{H}$ NMR: $\delta 7.65(\mathrm{~m}, 4 \mathrm{H}), 7.41(\mathrm{~m}, 6 \mathrm{H}), 5.84(\mathrm{~m}, 1 \mathrm{H}), 5.07(\mathrm{~m}, 2 \mathrm{H})$, $4.93(\mathrm{~s}, 1 \mathrm{H}), 4.80(\mathrm{~m}, 2 \mathrm{H}), 3.80(\mathrm{t}, J=6.9 \mathrm{~Hz}, 2 \mathrm{H}), 2.82(\mathrm{dd}, J=15,7.5 \mathrm{~Hz}, 1 \mathrm{H}), 2.58$ (dd, $J=15,5.4 \mathrm{~Hz}, 1 \mathrm{H}), 2.35$ (dt, $J=13.2,6.6 \mathrm{~Hz}, 2 \mathrm{H}), 2.10(\mathrm{~s}, 3 \mathrm{H}), 1.73(\mathrm{~s}, 3 \mathrm{H}) \mathrm{ppm}$. ${ }^{13}$ C NMR: $\delta 206.9,145.7,135.4,135.3,132.9,130.6,130.5,128.0,116.8,112.5,73.5$, 62.9, 50.5, 37.1, 31.2, 17.6 ppm. IR (neat): 3079, 2924, 2878, 1714, 1087, $719 \mathrm{~cm}^{-1}$. HRMS: Calcd. for $\mathrm{C}_{23} \mathrm{H}_{28} \mathrm{O}_{3} \mathrm{SiNa} 403.1680\left(\mathrm{M}+\mathrm{Na}^{+}\right)$. Found: 403.1699 .

\footnotetext{
${ }^{\mathrm{XXV}}$ Unfortunately only ${ }^{1} \mathrm{H}$ NMR spectral data is available. Due to the compounds unsuitability in the synthetic strategy it was deemed of little value to resynthesise this derivative for characterisation purposes only.
} 
4-(But-3-en-1-oxydiphenylsilyloxy)hex-5-en-2-one (66b). Dichlorodiphenylsilane (330 $\mu \mathrm{l}, 1.57 \mathrm{mmol})$ produced $66 \mathrm{~b}(374 \mathrm{mg}, 1.02 \mathrm{mmol})$ in an overall yield of $65 \% .{ }^{1} \mathrm{H}$ NMR: $\delta 7.65(\mathrm{~m}, 4 \mathrm{H}), 7.41(\mathrm{~m}, 6 \mathrm{H}), 5.50(\mathrm{~m}, 2 \mathrm{H}), 5.09(\mathrm{~m}, 4 \mathrm{H}), 4.84(\mathrm{dt}, J=12.9,6.0 \mathrm{~Hz}$, $1 \mathrm{H}), 3.81(\mathrm{t}, J=6.9 \mathrm{~Hz}, 2 \mathrm{H}), 2.79(\mathrm{dd}, J=15.0,6.6 \mathrm{~Hz}, 1 \mathrm{H}), 2.61(\mathrm{dd}, J=15.4,5.7 \mathrm{~Hz}$, $1 \mathrm{H}), 2.35$, (dt, $J=13.8,6.9 \mathrm{~Hz}, 2 \mathrm{H}), 2.11(\mathrm{~s}, 3 \mathrm{H}) \mathrm{ppm} .{ }^{13} \mathrm{C}$ NMR: $\delta 206.7,139.5,135.4$, $135.3,130.6,128.1,128.0,116.8,115.5,70.8,63.0,51.8,37.1,31.3$ ppm. IR (neat): 3071, 3003, 2874, 1715, 1115, 1079, 914, $717 \mathrm{~cm}^{-1}$. HRMS: Calcd. for $\mathrm{C}_{22} \mathrm{H}_{26} \mathrm{O}_{3} \mathrm{SiNa}$ : $389.1542\left(\mathrm{M}+\mathrm{Na}^{+}\right)$. Found: 389.1543 .

\section{General procedure for the formation of diisopropylsilylethers (66c) and (66d)}

To a stirred solution of but-3-en-1-oxydiisopropylsilylchloride (68b) (1.2 mmol), the appropriate $\beta$-hydroxyketone $(1 \mathrm{mmol})$, and DMAP $(0.05 \mathrm{mmol})$ in $\mathrm{CH}_{2} \mathrm{Cl}_{2}(5 \mathrm{~mL})$ was added $\mathrm{Et}_{3} \mathrm{~N}$ (1.2 mmol) dropwise. The resulting solution was stirred at room temperature for $18 \mathrm{~h}$ then quenched with satd. aq. $\mathrm{NaHCO}_{3}$ and extracted twice with $\mathrm{Et}_{2} \mathrm{O}$. The organic extracts were washed with water, brine, dried over $\mathrm{MgSO}_{4}$, filtered and concentrated under reduced pressure. The residual oil was purified by flash chromatography (20:1 hexanes:EtOAc) to give the requisite diisopropylsilylethers.

4-(But-3-en-1-oxydiisopropylsilyloxy)-5-methylhex-5-en-2-one (66c).

$\beta-$ Hydroxyketone $24 \mathrm{a}(329 \mathrm{mg}, 2.57 \mathrm{mmol})$ gave $66 \mathrm{c}(687 \mathrm{mg}, 86 \%)$ as a colourless oil. ${ }^{1} \mathrm{H}$ NMR: $\delta 5.84(\mathrm{~m}, 1 \mathrm{H}), 5.04(\mathrm{~m}, 2 \mathrm{H}), 4.81(\mathrm{~s}, 1 \mathrm{H}), 4.76(\mathrm{t}, J=6.3 \mathrm{~Hz}, 1 \mathrm{H}), 3.74(\mathrm{t}, J=6.9$ $\mathrm{Hz}, 2 \mathrm{H}), 2.74$ (dd, $J=15.0,7.2 \mathrm{~Hz}, 1 \mathrm{H}), 2.57$ (dd, $J=14.0,5.4 \mathrm{~Hz}, 1 \mathrm{H}), 2.30$, (dt, $J=$ 13.8, 6.6 Hz, 2H), 2.17 (s, 3H), $1.73(\mathrm{~s}, 3 \mathrm{H}), 1.02(\mathrm{~m}, 14 \mathrm{H}) \mathrm{ppm} .{ }^{13} \mathrm{C}$ NMR: $\delta 207.4$, $146.5,135.6,116.6,111.8,73.0,62.8,50.9,37.5,31.7,17.6,17.5,12.5,12.4$ ppm. IR (neat): 2945, 2867, 1716, 1085, 1060, 999, 883, $688 \mathrm{~cm}^{-1}$. HRMS: Calcd. for $\mathrm{C}_{17} \mathrm{H}_{32} \mathrm{O}_{3} \mathrm{SiNa} 335.2012\left(\mathrm{M}+\mathrm{Na}^{+}\right)$. Found: 335.2013. 
4-(But-3-en-1-oxydiisopropylsilyloxy)hex-5-en-2-one (66d). $\beta$-Hydroxyketone $24 \mathrm{~b}$ (243 mg, $2.13 \mathrm{mmol}$ ) gave $66 \mathrm{~d}(521 \mathrm{mg}, 82 \%)$ as a colourless oil. ${ }^{1} \mathrm{H}$ NMR: $\delta 5.84(\mathrm{~m}$, 2H), $5.20(\mathrm{~m}, 4 \mathrm{H}), 4.78(\mathrm{dt}, J=12.3,6.3 \mathrm{~Hz}, 1 \mathrm{H}), 3.75(\mathrm{t}, J=6.9 \mathrm{~Hz}, 2 \mathrm{H}), 2.74$ (dd, $J=$ $15.3,6.6 \mathrm{~Hz}, 1 \mathrm{H}), 2.57(\mathrm{dd}, J=15.3,6.3 \mathrm{~Hz}, 1 \mathrm{H}), 2.29$ (dt, $J=13.5,6.6 \mathrm{~Hz}, 2 \mathrm{H}), 2.16$ (s, $3 \mathrm{H}), 1.01(\mathrm{~m}, 14 \mathrm{H}) \mathrm{ppm} .{ }^{13} \mathrm{C}$ NMR: $\delta 207.2,140.3,135.6,116.6,114.8,70.2,62.8,52.2$, $37.5,31.7,17.6,17.5,12.5,12.4$ ppm. IR (neat): 2944, 2867, 1717, 1087, 990, 883, 689 $\mathrm{cm}^{-1}$. HRMS: Calcd. for $\mathrm{C}_{16} \mathrm{H}_{30} \mathrm{O}_{3} \mathrm{SiNa} 321.1847\left(\mathrm{M}+\mathrm{Na}^{+}\right)$. Found: 321.1856 .

4-(But-3-en-1-oxydimethylsilyloxy)hex-5-en-2-one (66e). To a stirred solution of 3buteneoxydimethylsilylchloride (68c) $(386 \mathrm{mg}, 2.34 \mathrm{mmol})$ and $\beta$-hydroxyketone 24a (243 mg, $2.13 \mathrm{mmol}$ ) in $\mathrm{CH}_{2} \mathrm{Cl}_{2}(10.5 \mathrm{~mL})$ was added $\mathrm{Et}_{3} \mathrm{~N}(386 \mu \mathrm{l}, 2.77 \mathrm{mmol}$ ) dropwise. The solution was then stirred at room temperature for $3 \mathrm{~h}$ before being quenched with satd. aq. $\mathrm{NaHCO}_{3}$, extracted twice with $\mathrm{Et}_{2} \mathrm{O}$, and the organic extracts washed with water, brine, and dried over $\mathrm{MgSO}_{4}$. Filtration and concentration gave a residual oil, which was purified by gradient flash chromatography (10:1 to 5:1 hexanes:EtOAc) to give $68 \mathrm{e}(396 \mathrm{mg}, 77 \%)$ as a colourless oil. ${ }^{1} \mathrm{H}$ NMR: $\delta 5.85(\mathrm{~m}, 2 \mathrm{H})$, $5.26(\mathrm{~m}, 4 \mathrm{H}), 4.75(\mathrm{~m}, 1 \mathrm{H}), 3.70(\mathrm{t}, J=6.9 \mathrm{~Hz}, 2 \mathrm{H}), 2.76(\mathrm{dd}, J=15.3,7.8 \mathrm{~Hz}, 1 \mathrm{H}), 2.53$ (dd, $J=15.3,5.1 \mathrm{~Hz}, 1 \mathrm{H}), 2.31(\mathrm{dt}, J=13.8,6.9 \mathrm{~Hz}, 2 \mathrm{H}), 2.19(\mathrm{~s}, 3 \mathrm{H}), 0.15(\mathrm{~s}, 3 \mathrm{H}), 0.13$ (s, 3H) ppm. ${ }^{13} \mathrm{C}$ NMR: $\delta 206.9,139.7,135.1,116.5,114.6,69.9,62.0,51.3,36.9,31.4$, 2.7, -2.8 ppm. IR (neat): 2962, 2873, 1718, 1257, 1072, 1034, $796 \mathrm{~cm}^{-1}$.

\section{General procedure for $R C M$ reactions}

To a solution of the diene $(1 \mathrm{mmol})$ in $\mathrm{CH}_{2} \mathrm{Cl}_{2}(100 \mathrm{~mL})$ was added tricyclohexylphosphine[1,3-bis(2,4,6-trimethylphenyl)-4,5-dihydroimidazol-2-ylidene[benzylidine]ruthenium (IV) dichloride (62) $\left(0.09 \mathrm{mmol}\right.$ for $\mathrm{R}^{2}=\mathrm{H}, 0.12 \mathrm{mmol}$ for $\mathrm{R}^{2}=$ $\mathrm{Me})$. The reaction mixture was stirred for $8-12 \mathrm{~h}$ at room temperature, the solvent was removed under reduced pressure and the dark residue filtered through a short pad of silica gel (10:1 hexanes:EtOAc). The filtered solution was then stirred with activated charcoal 
$(8.5 \mathrm{~g})$ for $24 \mathrm{~h}$. The mixture was filtered, concentrated in vacuo and purified by careful gradient flash chromatography (50:1 to 10:1 hexanes:EtOAc) to provide the cyclic olefin as a colourless oil.

(4R, 5Z)-4,8-O-diphenylsilanediyl-5-methyloct-5-en-2-one (65a). Diene 66a (270 mg, $0.71 \mathrm{mmol})$ gave $\mathbf{6 5 a}$ in $54 \%$ yield $(135 \mathrm{mg}) .{ }^{1} \mathrm{H}$ NMR: $\delta 7.64(\mathrm{~m}, 4 \mathrm{H}), 7.39(\mathrm{~m}, 6 \mathrm{H})$, $5.44(\mathrm{t}, J=8.1 \mathrm{~Hz}, 1 \mathrm{H}), 4.90(\mathrm{dd}, J=9.6 .3 .9 \mathrm{~Hz}, 1 \mathrm{H}), 4.05(\mathrm{~m}, 2 \mathrm{H}), 2.94(\mathrm{dd}, J=15.3$, $9.9 \mathrm{~Hz}, 1 \mathrm{H}), 2.93(\mathrm{~m}, 1 \mathrm{H}), 2.62(\mathrm{dd}, J=15.3,3.6 \mathrm{~Hz}, 1 \mathrm{H}), 2.26(\mathrm{~m}, 1 \mathrm{H}), 2.25(\mathrm{~s}, 3 \mathrm{H})$, 1.60 (s, 3H) ppm. ${ }^{13} \mathrm{C}$ NMR: $\delta$ 207.6, 140.8, 134.8, 133.8, 133.5, 130.5, 130.4, 128.1, 125.1, 71.7, 64.4, 49.6, 31.2, 30.9, 21.5 ppm. IR (neat): 3070, 2919, 2876, 1713, 1115, 1083, 716, $699 \mathrm{~cm}^{-1}$. HRMS: Cald for $\mathrm{C}_{21} \mathrm{H}_{25} \mathrm{O}_{3} \mathrm{Si} 353.1568\left(\mathrm{M}+\mathrm{H}^{+}\right)$. Found: 353.1565 .

(5Z)-4,8-O-diphenylsilanediyloct-5-en-2-one (65b). Diene 66b (80 mg, $0.22 \mathrm{mmol}$ ) gave 65b in $81 \%$ yield $(60 \mathrm{mg}) .{ }^{1} \mathrm{H}$ NMR: $\delta 7.65(\mathrm{~m}, 4 \mathrm{H}), 7.39(\mathrm{~m}, 6 \mathrm{H}), 5.73(\mathrm{~m}, 2 \mathrm{H})$, $5.13(\mathrm{~m}, 1 \mathrm{H}), 4.09(\mathrm{~m}, 1 \mathrm{H}), 3.94(\mathrm{~m}, 1 \mathrm{H}), 2.96(\mathrm{dd}, J=15.0,8.1 \mathrm{~Hz}, 1 \mathrm{H}), 2.64$ (dd, $J=$ 15.0, $4.5 \mathrm{~Hz}, 1 \mathrm{H}), 2.63(\mathrm{~m}, 1 \mathrm{H}), 2.42(\mathrm{~m}, 1 \mathrm{H}), 2.22(\mathrm{~s}, 3 \mathrm{H}) \mathrm{ppm} .{ }^{13} \mathrm{C}$ NMR: $\delta$ 207.3, 135.2 , 135.0, 134.8, 133.7, 130.5, 130.4, 129.3, 128.2, 128.1, 67.4, 63.7, 51.5, 31.3, 31.1 ppm. IR (neat): 3067, 3013, 2955, 2926, 2878, 1715, 1128, 1082, 720, $700 \mathrm{~cm}^{-1}$. HRMS: Calcd. for $\mathrm{C}_{20} \mathrm{H}_{23} \mathrm{O}_{3} \mathrm{Si} 339.1408\left(\mathrm{M}+\mathrm{H}^{+}\right)$. Found: 339.1411 .

(4R, 5Z)-4,8-O-di-(isopropyl)silanediyl-5-methyloct-5-en-2-one (65c). Diene 66c (110 $\mathrm{mg}, 0.35 \mathrm{mmol})$ gave $65 \mathrm{c}$ in $60 \%$ yield $(60 \mathrm{mg}) .{ }^{1} \mathrm{H}$ NMR: $\delta 5.42(\mathrm{~m}, 1 \mathrm{H}), 4.89(\mathrm{dd}, J=$ 9.6, 3.6 Hz, $1 \mathrm{H}), 3.9$ (m, $2 \mathrm{H}), 2.82$ (dd, $J=14.4,9.6 \mathrm{~Hz}, 1 \mathrm{H}), 2.72$ (m, $1 \mathrm{H}), 2.50$ (dd, $J$ $=14.7,3.6 \mathrm{~Hz}, 1 \mathrm{H}), 2.25(\mathrm{~s}, 3 \mathrm{H}), 2.24(\mathrm{~m} .1 \mathrm{H}), 1.74(\mathrm{~s}, 3 \mathrm{H}), 1.00(\mathrm{~m}, 14 \mathrm{H}) \mathrm{ppm} .{ }^{13} \mathrm{C}$ NMR: $\delta$ 208.1, 140.6, 125.0, 71.0, 64.3, 49.6, 31.6, 30.8, 21.1, 17.6, 17.5, 12.8, 12.5 ppm. IR (neat): 2943, 2866, 1715, 1112, 1036, 883, $693 \mathrm{~cm}^{-1}$. HRMS: Calcd for $\mathrm{C}_{15} \mathrm{H}_{29} \mathrm{O}_{3} \mathrm{Si}\left(\mathrm{M}+\mathrm{H}^{+}\right)$285.1891. Found: 285.1881. 
(5Z)-4,8-O-di-(isopropyl)silanediyloct-5-en-2-one (65d). Diene 66d (340 mg, 1.14 mmol) gave $65 \mathrm{~d}$ in $74 \%$ yield $(229 \mathrm{mg}) .{ }^{1} \mathrm{H}$ NMR: $\delta 5.72(\mathrm{~m}, 2 \mathrm{H}), 4.93(\mathrm{~m}, 1 \mathrm{H}), 3.91(\mathrm{~m}$, 2H), $2.80(\mathrm{dd}, J=14.7,8.4 \mathrm{~Hz}, 1 \mathrm{H}), 2.56(\mathrm{~m}, 1 \mathrm{H}), 2.54(\mathrm{dd}, J=14.7,4.8 \mathrm{~Hz}, 1 \mathrm{H}), 2.40$ (m, 1H), 2.23 (s, 3H), $1.02(\mathrm{~m}, 14 \mathrm{H}) \mathrm{ppm} .{ }^{13} \mathrm{C}$ NMR: $\delta 207.9,135.3,129.4,66.9,63.8$, $51.5,31.7,31.1,17.7,17.6,17.5,12.8,12.5$ ppm. IR (neat): 2944, 2867, 1715, 1120, 1011, $732 \mathrm{~cm}^{-1}$. HRMS: Calcd. for $\mathrm{C}_{14} \mathrm{H}_{27} \mathrm{O}_{3} \mathrm{Si} 271.1717\left(\mathrm{M}+\mathrm{H}^{+}\right)$. Found: 271.1724.

(5Z)-4,8-O-di-methylsilanediyloct-5-en-2-one (65e). (Unoptimised). To a solution of diene 66e $(60 \mathrm{mg}, 0.25 \mathrm{mmol})$ in $\mathrm{CH}_{2} \mathrm{Cl}_{2}(18 \mathrm{~mL})$ was added tricyclohexylkphosphine[1,3-bis(2,4,6-trimethylphenyl)-4,5-dihydroimidazol-2-ylidene-[benzylidine]ruthenium (IV) dichloride (62) (10 mg, $0.12 \mathrm{mmol})$ and the solution stirred at room temperature for $16 \mathrm{~h}$. The solvent was removed under reduced pressure, and the dark brown residue purified by gradient flash chromatography (50:1 to 10:1 hexanes:EtOAc) to give $\mathbf{6 5 e}$ (90 mg, 42\%). (Substrate characterised by ${ }^{1} \mathrm{H}$ NMR only). ${ }^{1} \mathrm{H}$ NMR: $\delta 5.78(\mathrm{~m}, 2 \mathrm{H}), 4.94$ (m, $1 \mathrm{H}), 3.94(\mathrm{~m}, 1 \mathrm{H}), 3.78(\mathrm{~m}, 1 \mathrm{H}), 2.81(\mathrm{dd}, J=15.0,8.4 \mathrm{~Hz}, 1 \mathrm{H}), 2.56(\mathrm{dd}, J=15.3,4.5$ $\mathrm{Hz}, 1 \mathrm{H}), 2.20$ (s, 3H), 0.15 (s, 3H), 0.12 (s, 3H) ppm.

But-3-ene-1-oxymethoxydi-tert-butylsilane (71). To a solution of di-tert-butylsilyl-bis(trifluoromethanesulfonate) $(500 \mu \mathrm{L}, 1.39 \mathrm{mmol})$ in $\mathrm{DMF}(5 \mathrm{~mL})$ at $-40^{\circ} \mathrm{C}$ was added dropwise a solution of but-3-en-1-ol (120 $\mu \mathrm{L}, 1.39 \mathrm{mmol})$ in DMF (5 mL). The cooling bath was removed and the reaction stirred for $2 \mathrm{~h}$ before being quenched with methanol then extracted twice with $\mathrm{Et}_{2} \mathrm{O}$. The combined organic fractions were washed three times with satd. aq. $\mathrm{NaHCO}_{3}$, once with brine and dried over $\mathrm{MgSO}_{4}$. Filtration and concentration under reduced pressure gave a residual oil which was purified by flash chromatography (50:1 hexanes:EtOAc) to give $71(240 \mathrm{mg}, 71 \%)$ as a colourless oil. ${ }^{1} \mathrm{H}$ NMR: $\delta 5.88(\mathrm{~m}, 1 \mathrm{H}), 5.08(\mathrm{~m}, 2 \mathrm{H}), 3.91(\mathrm{t}, J=6.6 \mathrm{~Hz}, 2 \mathrm{H}), 3.66(\mathrm{~s}, 3 \mathrm{H}), 2.36(\mathrm{dt}, J=$ 13.2, $6.6 \mathrm{~Hz}, 2 \mathrm{H}), 1.04$ (s, 18H) ppm. ${ }^{13} \mathrm{C}$ NMR: $\delta 135.7,116.6,63.7,52.3,37.7,28.1$, $21.4 \mathrm{ppm}$. 
But-3-ene-1-oxyallyloxy-di-tert-butylsilane (72). To a solution of di-tert-butylsilyl-bis(trifluoromethanesulfonate) $(300 \mu \mathrm{L}, 0.82 \mathrm{mmol})$ in DMF $(3 \mathrm{~mL})$ at $-40^{\circ} \mathrm{C}$ was added dropwise a solution of but-3-en-1-ol $(71 \mu \mathrm{L}, 0.82 \mathrm{mmol})$ in DMF $(1.5 \mathrm{~mL})$. The cooling bath was removed and the reaction allowed to gradually warm to room temperature. After $1 \mathrm{~h}$, pyridine ( $67 \mu \mathrm{L}, 0.82 \mathrm{mmol})$ then allyl alcohol $(112 \mu \mathrm{L}, 1.65 \mathrm{mmol})$ were added and the reaction stirred for a further $30 \mathrm{~min}$ before being quenched by pyridine $(67 \mu \mathrm{L}, 0.82$ $\mathrm{mmol}$ ). The solution was diluted with water, extracted twice with $\mathrm{Et}_{2} \mathrm{O}$, and the combined organic fractions washed three times with satd. aq. $\mathrm{NaHCO}_{3}$, once with brine and dried over $\mathrm{MgSO}_{4}$ before being filtered and the solvents removed under reduced pressure. The residual oil was purified by flash chromatography (hexanes) to give 72 (145 $\mathrm{mg}, 65 \%$ ) as a colourless oil. ${ }^{1} \mathrm{H}$ NMR: $\delta 5.92(\mathrm{~m}, 2 \mathrm{H}), 5.35(\mathrm{~m}, 1 \mathrm{H}), 5.10(\mathrm{~m}, 3 \mathrm{H}), 4.40$ (dd, $J=3.9$, $1.8 \mathrm{~Hz}, 2 \mathrm{H}), 3.88(\mathrm{t}, J=6.9 \mathrm{~Hz}, 2 \mathrm{H}), 2.34(\mathrm{~d}, \mathrm{t}, J=13.2,6.6 \mathrm{~Hz}, 2 \mathrm{H}), 1.04(\mathrm{~s}, 18 \mathrm{H}) \mathrm{ppm}$. ${ }^{13} \mathrm{C}$ NMR:137.6, 135.8, 116.6, 113.7, 64.6, 63.6, 37.7, 28.1, $21.4 \delta$ ppm. IR (neat): 2968 , $2933,2858,1093,913,825 \mathrm{~cm}^{-1}$.

4-tert-Butyldimethylsiloxy-5-methyl-5-hexen-2-one (75a). To a solution of 4-hydroxyl5-methy-hex-5-ene-2-one (24a) (300 mg, $2.34 \mathrm{mmol})$ in DMF (2.5 mL) was added tertbutyldimethylsilylchloride (388 mg, $2.58 \mathrm{mmol})$ and imidazole $(351 \mathrm{mg}, 5.15 \mathrm{mmol})$. After stirring at room temperature for $24 \mathrm{~h}$, the solution was quenched with water and extracted twice with $\mathrm{Et}_{2} \mathrm{O}$. The organic extracts were washed twice with satd. aq. $\mathrm{NaHCO}_{3}$, once with brine and dried over $\mathrm{MgSO}_{4}$ before being filtered and the solvent removed under reduced pressure. The residual oil was purified by gradient flash chromatography (hexanes to $50: 1$ : hexanes:EtOAc) to give $75 \mathbf{a}(413 \mathrm{mg}, 73 \%)$ as a colourless oil. ${ }^{1} \mathrm{H}$ NMR: $\delta 4.95$ (s, $\left.1 \mathrm{H}\right), 4.78$ (s, 1H), 4.54 (dd, $\left.J=8.4,3.9 \mathrm{~Hz}, 1 \mathrm{H}\right), 2.74$ (dd, $J=14.7,8.7 \mathrm{~Hz}, 1 \mathrm{H}), 2.39$ (dd, $J=18.3,3.9 \mathrm{~Hz}, 1 \mathrm{H}), 2.16$ (s, 3H), 1.69, (s, 3H), 0.85 (s, 9H), 0.02 (s, 3H), 0.00, (s, 3H) ppm. ${ }^{13} \mathrm{C}$ NMR: $\delta 207.8,147.0,111.5,73.7,50.6$, 32.0, 26.0, 18.3, 17.46, -4.6, -5.1 ppm. IR (neat): 2930, 2857, 1360, 1718, 1251, 1074, $835,776 \mathrm{~cm}^{-1}$. HRMS: Calcd. for $\mathrm{C}_{13} \mathrm{H}_{27} \mathrm{O}_{2} \mathrm{Si} 243.1774\left(\mathrm{M}+\mathrm{H}^{+}\right)$: Found. 243.1780 . 
4-tert-Butyldimethylsiloxyhex-5-en-2-one (75b). To a solution of 4-hydroxy-5-hexen-2one (24b) (300 mg, $2.63 \mathrm{mmol})$ in DMF (2 mL) was added tert-butyldimethylsilylchloride (436 mg, $2.89 \mathrm{mmol}$ ) and imidazole (394 mg, $5.78 \mathrm{mmol}$ ). After stirring at room temperature for $24 \mathrm{~h}$, the solution was quenched with satd. aq. $\mathrm{NH}_{4} \mathrm{Cl}$ and extracted twice with $\mathrm{Et}_{2} \mathrm{O}$. The organic extracts were washed with brine, dried over $\mathrm{MgSO}_{4}$, filtered and the solvents removed under reduced pressure. The residual oil was purified by flash chromatography (50:1 hexanes:EtOAc) to give $\mathbf{7 5 b}(446 \mathrm{mg}, 74 \%)$ as a colourless oil. ${ }^{1} \mathrm{H}$ NMR: $\delta 5.78(\mathrm{dd}, J=12.0,6.0 \mathrm{~Hz}, 1 \mathrm{H}), 5.15(\mathrm{~m}, 1 \mathrm{H}), 5.01(\mathrm{~m}, 1 \mathrm{H}), 4.55(\mathrm{~m}, 1 \mathrm{H}), 2.43$ (dd, $J=15.0,9.0 \mathrm{~Hz}, 1 \mathrm{H}), 2.43(\mathrm{dd}, J=15.0,3.0 \mathrm{~Hz}, 1 \mathrm{H}), 1.90(\mathrm{~s}, 3 \mathrm{H}), 0.83(\mathrm{~s}, 9 \mathrm{H}),-0.2$ (s, 6H) ppm. ${ }^{13} \mathrm{C}$ NMR: $\delta 207.4,140.6,114.5,70.7,51.9,32.0,26.0,18.3,-4.2,-4.9$ ppm. IR (neat): 2956, 2930, 2858, 1718, 1078, 834, $776 \mathrm{~cm}^{-1}$. HRMS: Calcd. for $\mathrm{C}_{12} \mathrm{H}_{25} \mathrm{O}_{2} \mathrm{SiNa}$ $229.1624\left(\mathrm{M}+\mathrm{Na}^{+}\right)$. Found: 229.1618 .

4-tert-Butyldiphenylsiloxy-5-methylhex-5-en-2-one (75c). To a solution of 4-hydroxy5-methylhex-5-en-2-one (24a) (437 mg, $3.4 \mathrm{mmol})$ in DMF (4.5 mL) was added tertbutyldiphenylsilylchloride ( $976 \mu \mathrm{L}, 3.75 \mathrm{mmol}$ ) and imidazole (464 mg, $6.82 \mathrm{mmol}$ ). After stirring at room temperature for $36 \mathrm{~h}$, the solution was quenched with water and extracted twice with $\mathrm{Et}_{2} \mathrm{O}$. The organic extracts were then washed twice with satd. aq. $\mathrm{NaHCO}_{3}$, once with brine and dried over $\mathrm{MgSO}_{4}$ before being filtered and the solvent removed under reduced pressure. The residual oil was purified by gradient flash chromatography (hexanes to 50:1: hexanes:EtOAc) to give $75 \mathrm{c}(920 \mathrm{mg}, 75 \%)$ as a colourless oil. ${ }^{1} \mathrm{H}$ NMR: $\delta 7.65(\mathrm{~m}, 4 \mathrm{H}), 7.38(\mathrm{~m}, 6 \mathrm{H}), 4.78(\mathrm{~s}, 1 \mathrm{H}), 4.72(\mathrm{~s}, 1 \mathrm{H}), 4.59$ (t, $J=6 \mathrm{~Hz}, 1 \mathrm{H}), 2.61(\mathrm{dd}, J=14.7,6.0 \mathrm{~Hz}, 1 \mathrm{H}), 2.52(\mathrm{dd}, J=14.7,6.6 \mathrm{~Hz}, 1 \mathrm{H}), 2.10$ (s, $3 \mathrm{H}), 1.68$ (s, 3H), 1.06 (s, 9H) ppm. ${ }^{13} \mathrm{C}$ NMR: $\delta 206.8,145.8,136.2,134.1,133.7,130.0$, 128.0, 127.8, 127.7, 112.6, 74.1, 50.8, 31.1, 27.2, 19.6, 17.4 ppm. IR (neat): 3072, 2931, $2857,1713,1427,1360,1162,1106,1063,739,700 \mathrm{~cm}^{-1}$. HRMS: Calcd. for $\mathrm{C}_{23} \mathrm{H}_{30} \mathrm{O}_{2} \mathrm{SiNa} 389.1922\left(\mathrm{M}+\mathrm{Na}^{+}\right)$. Found: 389.1907 . 
4-O-(Methoxymethyloxy)-5-methylhex-5-en-2-one (75d). To a solution of 4-hydroxy-5methylhex-5-ene-2-one (24a) $(1.0 \mathrm{~g}, 7.80 \mathrm{mmol})$, in $N, N$-diisopropylethylamine $(2.72 \mathrm{~mL})$ at $0{ }^{\circ} \mathrm{C}$ was added methoxymethylchloride $(593 \mu \mathrm{L}, 7.80 \mathrm{mmol})$ dropwise. The reaction was stirred for $3 \mathrm{~h}$, gradually warming to room temperature, before being quenched with water and extracted twice with $\mathrm{Et}_{2} \mathrm{O}$. The organic extracts were washed with satd. aq. $\mathrm{NaHCO}_{3}$, brine, dried over $\mathrm{MgSO}_{4}$, filtrated and concentrated under reduced pressure. The residual oil was purified by flash chromatography (5:1 hexanes:EtOAc) to give 75d (430 $\mathrm{mg}, 32 \%$, unoptimised) as a colourless oil. ${ }^{\mathrm{I}} \mathrm{H} \mathrm{NMR}: \delta 5.01(\mathrm{~s}, 1 \mathrm{H}), 4.94(\mathrm{t}, J=0.6 \mathrm{~Hz}$, $1 \mathrm{H}), 4.60$ (d, $J=6.6 \mathrm{~Hz}, 1 \mathrm{H}), 4.53$ (dd, $J=9.3,3.9 \mathrm{~Hz}, 1 \mathrm{H}), 4.48(\mathrm{~d}, J=6.6 \mathrm{~Hz}, 1 \mathrm{H})$, 3.34 (s, 3H), 2.82 (dd, $J=15.6,9.3 \mathrm{~Hz}, 1 \mathrm{H}), 2.46$ (dd, $J=15.9,3.9 \mathrm{~Hz}, 1 \mathrm{H}), 2.20(\mathrm{~s}, 3 \mathrm{H})$, 1.68 (s, 3H) ppm. ${ }^{13} \mathrm{C}$ NMR: $\delta 206.8,143.2,114.7,94.0,76.0,56.0,48.2,31.2,17.3$ ppm. IR (neat): 2947, 2824, 1715, 1096, 1026, $914 \mathrm{~cm}^{-1}$.

4-O-(Methoxymethyloxy)hex-5-en-2-one (75e). To a solution of 4-hydroxy-5-hexene-2one (24b) (350 mg, $3.07 \mathrm{mmol})$, in $N, N$-diisopropylethylamine (1.06 mL, $6.13 \mathrm{mmol})$ at $0{ }^{\circ} \mathrm{C}$ was added methoxymethylchloride $(221 \mu \mathrm{L}, 2.91 \mathrm{mmol})$ dropwise. The reaction was stirred for $3 \mathrm{~h}$, gradually warming to room temperature, before being quenched with water and extracted twice with $\mathrm{Et}_{2} \mathrm{O}$. The organic extracts were washed with satd. aq. $\mathrm{NaHCO}_{3}$, brine, dried over $\mathrm{MgSO}_{4}$, filtrated and concentrated under reduced pressure. The residual oil was purified by flash chromatography (5:1 hexanes:EtOAc) to give 75 e $(221 \mathrm{mg}, 48 \%)$ as a colourless oil. ${ }^{1} \mathrm{H}$ NMR: $\delta 5.71(\mathrm{dd}, J=10.5,7.8 \mathrm{~Hz}, 1 \mathrm{H}), 5.22(\mathrm{~m}, 2 \mathrm{H}), 4.67(\mathrm{~d}, J=$ $6.9 \mathrm{~Hz}, 1 \mathrm{H}), 4.52(\mathrm{~d}, J=6.9 \mathrm{~Hz}, 1 \mathrm{H}), 3.34(\mathrm{~s}, 3 \mathrm{H}), 2.80(\mathrm{dd}, J=15.9,8.7 \mathrm{~Hz}, 1 \mathrm{H}), 2.53$ (dd, $J=15.6,4.81 \mathrm{H}), 2.19$ (s, 3H) ppm, ${ }^{13} \mathrm{C}$ NMR: $\delta 206.3,136.9,117.8,94.1,73.4$, $55.6,49.2,31.1 \mathrm{ppm}$. IR (neat): 2935, 2894, 1716, 1027, $919 \mathrm{~cm}^{-1}$. 


\section{Representative procedure for aldol reactions}

To a solution of diisopropylamine or tetramethylpiperidine (1.05 mmol) in dry THF (3 $\mathrm{mL}$ ) cooled to $-78{ }^{\circ} \mathrm{C}$ was added butyllithium (1.00 mmol, $1.6 \mathrm{M}$ solution in hexanes) dropwise. After stirring at $-78^{\circ} \mathrm{C}$ for $10 \mathrm{~min}$, a solution of the ketone $(1.00 \mathrm{mmol})$ in THF ( $3 \mathrm{~mL}$ ) was added dropwise. Stirring was continued for $50 \mathrm{~min}$ before a solution of pivaldehyde $(1.20 \mathrm{mmol})$ in THF $(2 \mathrm{~mL})$ was added. The resultant solution was then

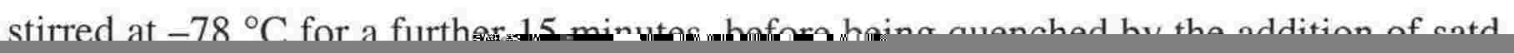


$0.92(\mathrm{~s}, 18 \mathrm{H}), 0.90(\mathrm{~s}, 9 \mathrm{H}), 0.88(\mathrm{~s}, 9 \mathrm{H}), 0.06(\mathrm{~s}, 6 \mathrm{H}), 0.05(\mathrm{~s}, 6 \mathrm{H}) \mathrm{ppm} .{ }^{13} \mathrm{C}$ NMR: $\delta$ $211.5,140.5,114.8,74.9,74.6,70.9,70.8,52.0,51.8,46.7,46.4,26.1,34.1,25.9,18.4$, $4.2,-4.8 \mathrm{ppm}$. IR (neat): $3500,2956,2858,1707,1076,835,776 \mathrm{~cm}^{-1}$.

7-tert-Butyldiphenylsiloxy-3-hydroxy-2,2,8-trimethylnon-8-ene-5-one (78c). Ketone $75 \mathrm{c}(755 \mathrm{mg}, 3.11 \mathrm{mmol})$ gave $78 \mathrm{c}(681 \mathrm{mg}, 67 \%)$ as a colourless oil after purification by gradient flash chromatography (50:1 to 10:1 hexanes:EtOAc). Spectral data reported for the racemic product. ${ }^{1} \mathrm{H}$ NMR: $\delta 4.99$ (s, $\left.1 \mathrm{H}\right), 4.82(\mathrm{~s}, 1 \mathrm{H}), 4.56(\mathrm{dd}, J=8.7,3.9 \mathrm{~Hz}, 1 \mathrm{H})$, $3.71(\mathrm{~m}, 1 \mathrm{H}), 3.05(\mathrm{~d}, J=2.7 \mathrm{~Hz}, 1 \mathrm{H}), 2.84-2.43(\mathrm{~m}, 4 \mathrm{H}), 1.73(\mathrm{~s}, 3 \mathrm{H}), 0.92(\mathrm{~s}, 9 \mathrm{H})$, 0.89 (s, 9H), 0.06 (s, 3H), 0.03 (s, 3H) ppm. ${ }^{13} \mathrm{C}$ NMR: $\delta 211.8,211.5,146.8,111.7,75.0$, $74.6,73.7,73.6,50.5,46.7,46.5,34.3,34.2,26.0,25.9,18.7,17.6,-4.6,-5.1$ ppm. IR (neat): $3507,2955,2858,1709,1072,835,776 \mathrm{~cm}^{-1}$.

7-O-(Methoxymethyloxy)-3-hydroxy-2,2,8-trimethylnon-8-ene-5-one (78d). Ketone 75d (390 mg, $2.26 \mathrm{mmol}$ ) gave 78d (325 mg, 56\%) as a colourless oil after purification by gradient flash chromatography ( $5: 1$ to $3: 1$ hexanes:EtOAc) on a silica gel column neutralised with $0.01 \% \mathrm{Et}_{3} \mathrm{~N}$ in 5:1 hexane:EtOAc. Spectral data reported for the racemic product. ${ }^{1} \mathrm{H}$ NMR: $\delta 5.05$ (s, $\left.1 \mathrm{H}\right), 4.98(\mathrm{~s}, 1 \mathrm{H}), 4.62(\mathrm{~d}, J=6.6 \mathrm{~Hz}, 1 \mathrm{H}), 4.60(\mathrm{~m}, 1 \mathrm{H})$, $4.50(\mathrm{~d}, J=6.6 \mathrm{~Hz}, 1 \mathrm{H}), 3.76(\mathrm{~m}, 1 \mathrm{H}), 3.36(\mathrm{~d}, J=2.4 \mathrm{~Hz}, 1 \mathrm{H}), 2.89(\mathrm{~m}, 1 \mathrm{H}), 2.74-2.46$ $(\mathrm{m}, 3 \mathrm{H}), 1.71(\mathrm{~s}, 3 \mathrm{H}), 0.93$ (s, 9H) ppm. ${ }^{13} \mathrm{C}$ NMR: $\delta 206.2,114.8,94.0,76.0,75.8,75.1$, $74.8,56.1,48.1,48.0,45.8,32.4,25.9,17.4$ ppm. (The compound decomposed before further spectral data could be obtained).

3-Benzyloxy-7-tert-butyldimethylsiloxy-2,2,8-trimethylnon-8-ene-5-one (80b). To a solution of aldol adduct $78 \mathrm{~b}(662 \mathrm{mg}, 2.0 \mathrm{mmol})$ in cyclohexane $(20 \mathrm{~mL})$ cooled to $0{ }^{\circ} \mathrm{C}$ was added benzyltrichloroacetimidate (79) $(385 \mu \mathrm{l}, 2.12 \mathrm{mmol})$ followed by freshly 
distilled trifluoromethanesulfonic acid $(8.8 \mu \mathrm{l}, 0.10 \mathrm{mmol})$ dropwise. A cream precipitate was immediately observed. The resulting mixture was stirred at room temperature for 30 min before being quenched with water then extracted twice with hexanes. The organic extracts were washed with satd. aq. $\mathrm{NaHCO}_{3}$, brine, dried over $\mathrm{MgSO}_{4}$, filtered and concentrated under reduced pressure. Purification of the crude material by gradient flash chromatography (hexanes to $50: 1$; hexanes:EtOAc) gave $\mathbf{8 0 b}(601 \mathrm{mg}, 72 \%)$ as a colourless oil. Spectral data reported for the racemic product. ${ }^{1} \mathrm{H}$ NMR: $\delta 7.33(\mathrm{~m}, 5 \mathrm{H})$, 4.97 (s, 1H), 4.79 (s, 1H), $4.55(\mathrm{~m}, 3 \mathrm{H}), 3.76(\mathrm{~m}, 1 \mathrm{H}), 2.84-2.37(\mathrm{~m}, 4 \mathrm{H}), 1.74(\mathrm{~s}, 3 \mathrm{H})$, 0.95 (s, 9H), 0.87 (s, 9H), 0.05 (s, 3H), 0.04 (s, 3H) ppm. ${ }^{13} \mathrm{C}$ NMR: $\delta$ 208.9, 208.6, $147.1,147.0,139.4,139.3,128.5,128.1,127.7,127.5,127.2,111.6,111.5,82.9,82.8$, $74.4,74.0,73.4,73.1,51.0,47.1,46.3,35.9,35.8,26.4,26.3,26.0,17.6,17.5,-4.6,-5.0$ ppm. IR (neat): $2955,2859,1717,1073,835 \mathrm{~cm}^{-1}$.

3-Benzyloxy-7-tert-butyldiphenylsiloxy-2,2,8-trimethylnon-8-ene-5-one (80c). To a solution of aldol adduct $78 \mathrm{c}(172 \mathrm{mg}, 0.48 \mathrm{mmol})$ in cyclohexane $(5 \mathrm{~mL})$ cooled to $0{ }^{\circ} \mathrm{C}$ was added benzyltrichloroacetimidate $(92 \mu \mathrm{l}, 0.51 \mathrm{mmol})$ followed by freshly distilled trifluoromethanesulfonic acid $(5 \mu \mathrm{l}, 0.06 \mathrm{mmol})$ dropwise. A cream precipitate was immediately observed and the resulting mixture was stirred at room temperature for 30 min before being quenched with water. The solution was then extracted with hexanes and the organic extracts washed with satd. aq. $\mathrm{NaHCO}_{3}$, brine, dried over $\mathrm{MgSO}_{4}$, filtered and concentrated under reduced pressure. Purification of the crude material by gradient flash chromatography (hexanes to $50: 1$; hexanes:EtOAc) gave $80 \mathrm{c}(143 \mathrm{mg}, 71 \%$ ) as a colourless oil. Spectral data is reported for the racemic product. ${ }^{1} \mathrm{H}$ NMR: $\delta 7.69(\mathrm{~m}, 4 \mathrm{H})$, $7.34(\mathrm{~m}, 11 \mathrm{H}), 4.80(\mathrm{~m}, 1 \mathrm{H}), 4.74(\mathrm{~m}, 1 \mathrm{H}), 4.62(\mathrm{~m}, 1 \mathrm{H}), 4.46(\mathrm{~m}, 2 \mathrm{H}), 3.62(\mathrm{dd}, J=7.8$, $3.9 \mathrm{~Hz}, 1 \mathrm{H}), 2.71-2.25(\mathrm{~m}, 4 \mathrm{H}), 1.71(\mathrm{~s}, 3 \mathrm{H}), 1.08(\mathrm{~s}, 9 \mathrm{H}), 0.89(\mathrm{~s}, 3 \mathrm{H}), 0.88$ (s, 3H) ppm. ${ }^{13} \mathrm{C}$ NMR: $\delta$ 208.0, 207.8, 145.8, 145.6, 139.4, 136.3, 136.2, 134.1, 133.6, 130.0, $129.9,128.4,127.9,127.8,127.7,127.5,112.7,82.8,82.6,74.1,74.0,73.9,51.1,50.9$, $46.0,45.8,35.8,27.2,26.4,26.3,19.6,17.5,17.4$ ppm. IR (neat): 3070, 2956, 2859, $1714,1106,1067,736,700 \mathrm{~cm}^{-1}$. 
4-(But-3-en-1-oxydiisopropylsilyloxy)-7-hydroxy-8,8-dimethyl-non-8-ene-5-one (82).

To a solution tetramethylpiperidine $(60 \mu \mathrm{L}, 0.36 \mathrm{mmol})$ in dry THF (1.5 mL) cooled to $-78^{\circ} \mathrm{C}$ was added $n$-BuLi (222 $\mu \mathrm{L}, 0.36 \mathrm{mmol}, 1.6 \mathrm{M}$ solution in hexanes). After stirring at $-78^{\circ} \mathrm{C}$ for $10 \mathrm{~min}$ a solution of the ketone $\mathbf{6 6} \mathrm{d}(106 \mathrm{mg}, 0.36 \mathrm{mmol})$ in THF $(2 \mathrm{~mL})$ was added dropwise. Stirring was continued for $90 \mathrm{~min}$ before pivaldehyde ( $58 \mu \mathrm{L}, 0.53$ mmol) was added dropwise, and the solution was stirred at $-78^{\circ} \mathrm{C}$ for a further $30 \mathrm{~min}$ before being quenched with water. The solution was then extracted twice with $\mathrm{Et}_{2} \mathrm{O}$ and the organic extracts washed with brine, dried over $\mathrm{MgSO}_{4}$ and the solvent removed under reduced pressure. The residual oil was purified by gradient flash chromatography $(50: 1$ to 10:1 hexanes:EtOAc) to give $\mathbf{8 2}(80 \mathrm{mg}, 59 \%)$ as a colourless oil. Spectral data reported for the racemic product. ${ }^{1} \mathrm{H}$ NMR: $\delta 5.84(\mathrm{~m}, 2 \mathrm{H}), 5.12(\mathrm{~m}, 4 \mathrm{H}), 4.83(\mathrm{~m}, 1 \mathrm{H}), 3.78(\mathrm{t}, J$ $=6.6 \mathrm{~Hz}, 2 \mathrm{H}), 2.93(\mathrm{~d}, J=3 \mathrm{~Hz}, 1 \mathrm{H}), 2.78-2.52(\mathrm{~m}, 4 \mathrm{H}), 2.33(\mathrm{dt}, J=13.5,6.6 \mathrm{~Hz}, 2 \mathrm{H})$, $1.04(\mathrm{~s}, 6 \mathrm{H}), 1.03(\mathrm{~s}, 6 \mathrm{H}), 1.00(\mathrm{~m}, 2 \mathrm{H}), 0.92$ (s, 9H) ppm. ${ }^{13} \mathrm{C}$ NMR: $\delta 210.8,139.9$, $135.3,116.5,114.8,114.7,74.6,70.0,69.9,62.6,52.0,51.8,46.0,45.8,37.2,34.1,34.0$, 25.6, 17.4, 17.3, 17.1, 17.0, 12.2 ppm. IR (neat): 3500, 2946, 2868, 1707, 1092, 1094, $885,691 \mathrm{~cm}^{-1}$.

(8Z)-7,11- $O$-diisopropylsilanediyl-3-hydroxy-2,2-dimethyl-undec-8-ene-5-one (81d). Strategy A. To a solution of diene $\mathbf{8 2 d}(60 \mathrm{mg}, 0.16 \mathrm{mmol})$ in $\mathrm{CH}_{2} \mathrm{Cl}_{2}(10 \mathrm{~mL})$ was added tricyclohexylphosphine[1,3-bis(2,4,6-trimethylphenyl)-4,5-dihydroimidazol-2ylidene-[benzylidine]ruthenium (IV) dichloride (62) (13 $\mathrm{mg}, 0.016 \mathrm{mmol}$ ) and the reaction stirred for $8 \mathrm{~h}$ at room temperature. The solvent was then removed under reduced pressure and the resultant brown oil purified rigorously three times by flash chromatography (10:1 hexanes:EtOAc) to give $\mathbf{8 1 d}(43 \mathrm{mg}, 77 \%)$ as a colourless oil. Spectral data is reported for the racemic product. ${ }^{1} \mathrm{H}$ NMR: $\delta 5.71(\mathrm{~m}, 2 \mathrm{H}), 4.93(\mathrm{~m}, 1 \mathrm{H})$, $3.88(\mathrm{~m}, 2 \mathrm{H}) 3.71(\mathrm{~m}, 1 \mathrm{H}), 3.05(\mathrm{~d}, J=3.3 \mathrm{~Hz}, 1 \mathrm{H}), 3.00(\mathrm{~d}, J=3.0 \mathrm{~Hz}, 1 \mathrm{H}), 2.87-2.32$ $(\mathrm{m}, 6 \mathrm{H}), 1.00(\mathrm{~m}, 14 \mathrm{H}), 0.91(\mathrm{~s}, 3 \mathrm{H}) \mathrm{ppm} .{ }^{13} \mathrm{C}$ NMR: $\delta 211.8,211.7,135.1,135.0,129.5$, $129.4,75.1,74.7,67.1,67.0,63.7,51.3,51.2,46.5,46.2,34.3,31.1,25.0,17.7,17.6$, $17.5,12.7,12.5$ ppm. IR (neat): $3502,2945,2869,1706,1112,1064,1004,695 \mathrm{~cm}^{-1}$. 
(8Z)-7,11-O-diisopropylsilanediyl-3-hydroxy-2,2-dimethyl-undec-8-ene-5-one (81d).

Strategy B. To a solution of tetramethylpiperidine $(143 \mu \mathrm{L}, 0.85 \mathrm{mmol})$ in dry THF (3 $\mathrm{mL})$ cooled to $-78^{\circ} \mathrm{C}$ was added $n$-BuLi $(529 \mu \mathrm{L}, 0.86 \mathrm{mmol}, 1.6 \mathrm{M}$ solution in hexanes). After stirring at $-78{ }^{\circ} \mathrm{C}$ for $10 \mathrm{~min}$ a solution of bis-silyl ether $65 \mathbf{d}(229 \mathrm{mg}, 0.86 \mathrm{mmol})$ in THF ( $3 \mathrm{~mL}$ ) was added dropwise. Stirring was continued for $45 \mathrm{~min}$ before pivaldehyde $(110 \mu \mathrm{L}, 1.02 \mathrm{mmol})$ was added dropwise and the resultant solution stirred for a further $15 \mathrm{~min}$ before being quenched by the addition of water. The solution was then extracted twice with $\mathrm{Et}_{2} \mathrm{O}$ and the organic extracts washed with brine, dried over $\mathrm{MgSO}_{4}$ and the solvent removed under reduced pressure. The residual product was purified by flash chromatography (10:1 hexanes:EtOAc) to give 81d (90 mg, 30\%, unoptimised) as a colourless oil. Spectral data matched that reported above. 


\section{6: Experimental for Chapter 6:}

(R)-4-Hydroxy-5-methyl-5-hexen-2-one (24). L-proline (438 mg, $3.76 \mathrm{mmol}$ ) was added to a solution of DMSO:acetone $(4: 1)(125 \mathrm{~mL})$ and the resulting suspension stirred for $15 \mathrm{~min}$ at room temperature. Freshly distilled methacrolein $(1.04 \mathrm{~mL}, 12.5 \mathrm{mmol})$ was then added. After being stirred at room temperature overnight, the resulting yellow solution was quenched with satd. aq. $\mathrm{NH}_{4} \mathrm{Cl}$, extracted twice with $\mathrm{Et}_{2} \mathrm{O}$ and the organic extracts washed with brine, dried over $\mathrm{MgSO}_{4}$ and filtered. The solvent was removed under reduced pressure and the residual oil purified by flash chromatography on a silica gel column neutralised with $0.01 \% \mathrm{Et}_{3} \mathrm{~N}$ (3:1 hexanes:EtOAc) to give $24(611 \mathrm{mg}, 38 \%$ ) which was used immediately. ${ }^{203}$ The enantiomeric excess of this reaction was determined by silylation followed by chiral GC analysis, as detailed below.

(R)-4-tert-Butyldimethylsilyl-5-methyl-hex-5-en-2-one. To a solution of the $\beta$-hydroxy ketone 24 (200 mg, $1.56 \mathrm{mmol})$ in DMF (3 mL) was added tert-butyldimethylsilylchloride (259 mg, $1.72 \mathrm{mmol})$ and imidazole $(212 \mathrm{mg}, 3.12 \mathrm{mmol})$. After stirring at room temperature for $24 \mathrm{~h}$, the solution was quenched with water, extracted twice with $\mathrm{Et}_{2} \mathrm{O}$, and the organic extracts washed with brine, dried over $\mathrm{MgSO}_{4}$, filtered and the solvents removed under reduced pressure. The residual oil was purified by flash chromatography (50:1 hexanes: EtOAc) to give $(R)$-4-tertbutyldimethylsilyl-5-methyl-hex-5-en-2-one (202 mg); 74\% ee determined using a $60 \mathrm{~m} \times 0.25 \mathrm{~mm}$ Cyclodex-B ${ }^{\mathrm{TM}}$ column and the following conditions: Inlet: Split $275^{\circ} \mathrm{C}$; Detector: FID $320^{\circ} \mathrm{C}$; Carrier gas: hydrogen; Flow: $35.8 \mathrm{~cm}^{3} \mathrm{~s}^{-1}$; Temperature programme: Isothermal at $75^{\circ} \mathrm{C}$. ${ }^{1} \mathrm{H}$ NMR: $\delta 4.95(\mathrm{~s}, 1 \mathrm{H})$, $4.78(\mathrm{~s}, 1 \mathrm{H}), 4.54(\mathrm{dd}, J=8.4,3.9 \mathrm{~Hz}, 1 \mathrm{H}), 2.74(\mathrm{dd}, J=14.7,8.7 \mathrm{~Hz}, 1 \mathrm{H}), 2.39(\mathrm{dd}, J=$ $18.3,3.9, \mathrm{~Hz}, 1 \mathrm{H}), 2.16$ (s, 3H), 1.69, (s, 3H), 0.85 (s, 9H), 0.02 (s, 3H), 0.00, (s, 3H) ppm. ${ }^{13} \mathrm{C}$ NMR: $\delta 207.8,147.0,111.5,73.7,50.6,32.0,26.0,18.3,17.46,-4.6,-5.1 \mathrm{ppm}$. IR (neat): 2930, 2857, 1360, 1718, 1251, 1074, 835, $776 \mathrm{~cm}^{-1}$. HRMS: Calcd for $\mathrm{C}_{13} \mathrm{H}_{27} \mathrm{O}_{2} \mathrm{Si} 243.1774\left(\mathrm{M}+\mathrm{H}^{+}\right)$. Found. 243.1780. 


\section{Representative procedure for aldol reactions}

To a stirred solution of $\mathrm{Cy}_{2} \mathrm{OBTf}$ or $(-)-\mathrm{Ipc}_{2} \mathrm{BCl}(1.5 \mathrm{mmol})$ in $4 \mathrm{~mL}$ of anhydrous $\mathrm{Et}_{2} \mathrm{O}$ at $-78{ }^{\circ} \mathrm{C}$ or $0{ }^{\circ} \mathrm{C}$ respectively, was slowly added $\mathrm{Et}_{3} \mathrm{~N}(1.7 \mathrm{mmol})$. A solution of the ketone $(1 \mathrm{mmol})$ in $\mathrm{Et}_{2} \mathrm{O}(4 \mathrm{~mL})$ was then added via cannula. The reaction mixture was stirred for 30 minutes, before being cooled to $-78{ }^{\circ} \mathrm{C}$ (if necessary) at which point pivaldehyde $(2 \mathrm{mmol})$ was added slowly dropwise. The solution was stirred at $-78{ }^{\circ} \mathrm{C}$ for $2 \mathrm{~h}$ then for $10 \mathrm{~h}$ at $-20^{\circ} \mathrm{C}$ before being quenched at $-20{ }^{\circ} \mathrm{C}$ by the addition of $\mathrm{pH} 7$ buffer solution, methanol and hydrogen peroxide (30\% solution). The solution was then stirred at room temperature for $3 \mathrm{~h}$ before being extracted twice with $\mathrm{Et}_{2} \mathrm{O}$. The organic extracts were washed with water, brine, dried over $\mathrm{MgSO}_{4}$, filtered and concentrated under reduced pressure. Purification of the crude material by gradient flash chromatography (50:1 to 10:1 hexanes:EtOAc) gave the aldol adducts as colourless oils.

\section{3-(3-Butenoxydiisopropylsilyloxy)-7-hydroxy-2,8,8-trimethyl-non-1-ene-5-one}

(90a/91a). (Entry 1, table 4): Reaction of $66 \mathbf{a}(50 \mathrm{mg}, 0.16 \mathrm{mmol})$ with (-)- $\mathrm{Ipc}_{2} \mathrm{BCl}$ gave 90a/91a in $93 \%(59 \mathrm{mg})$, ds $=2.2: 1$. Spectral data is reported for the combined diastereomers. ' H NMR: $\delta 5.86(\mathrm{~m}, 1 \mathrm{H}), 5.06(\mathrm{~m}, 2 \mathrm{H}), 5.00(\mathrm{~s}, 1 \mathrm{H}), 4.84(\mathrm{~s}, 1 \mathrm{H}), 4.78(\mathrm{t}$, $J=6.3 \mathrm{~Hz}, 1 \mathrm{H}), 3.74(\mathrm{~m}, 2 \mathrm{H}), 2.79-2.44(\mathrm{~m}, 4 \mathrm{H}), 2.31(\mathrm{dt}, J=13.5,6.9 \mathrm{~Hz}, 2 \mathrm{H}), 1.75$ $(\mathrm{s}, 3 \mathrm{H}), 1.03(\mathrm{~m}, 14 \mathrm{H}), 0.92$ (s, 9H) ppm. ${ }^{13} \mathrm{C}$ NMR: $\delta 211.2,211.1,146.4,135.5,116.6$, $112.0,111.9,74.9,74.8,72.9,62.9,50.8,50.7,46.2,46.1,37.5,34.3,26.0,25.9,17.6$, 17.5, 17.3, 12.5, 12.4 ppm. IR (neat): $3530,2948,2868,1709,1101,909 \mathrm{~cm}^{-1}$. HRMS:

Calcd. for $\mathrm{C}_{22} \mathrm{H}_{42} \mathrm{O}_{4} \mathrm{SiNa} 421.2734\left(\mathrm{M}+\mathrm{Na}^{+}\right)$. Found: 421.2744 .

\section{3-(3-Butenoxydiisopropylsilyloxy)-7-hydroxy-2,8,8-trimethyl-non-1-ene-5-one}

(90a/91a). (Entry 2, table 4). Reaction of (66a) (50 mg, $0.16 \mathrm{mmol})$ with $\mathrm{Cy}_{2} \mathrm{BOTf}$ gave 90a/91a in $97 \%$ (61 mg), ds $=1.2: 1$. 
3-(3-Butenoxydiphenylsilyloxy)-7-hydroxy-8,8-dimethylnon-1-ene-5-one (90b/91b). (Entry 3, table 4). Reaction of $\mathbf{6 6 b}(62 \mathrm{mg}, 0.17 \mathrm{mmol})$ with (-)- $\mathrm{Ipc}_{2} \mathrm{BCl}$ gave $\mathbf{9 0 b} / \mathbf{9 1 b}$ in $98 \%(75 \mathrm{mg})$, ds $=2: 1$. Spectral data is reported for the combined diastereomers. ${ }^{1} \mathrm{H}$ NMR: $\delta 7.65(\mathrm{~m}, 2 \mathrm{H}), 7.43(\mathrm{~m}, 3 \mathrm{H}), 5.89(\mathrm{~m}, 2 \mathrm{H}), 5.25(\mathrm{~m}, 4 \mathrm{H}), 4.84(\mathrm{dd} J=12.0,6.0$ $\mathrm{Hz}, 1 \mathrm{H}), 3.81(\mathrm{t}, J=12 \mathrm{~Hz}, 1 \mathrm{H}), 3.69(\mathrm{~m}, 1 \mathrm{H}), 2.9-2.4(\mathrm{~m}, 4 \mathrm{H}), 2.35$ (dt, $J=15.0,6.9$ $\mathrm{Hz}, 2 \mathrm{H}), 0.88$ (s, 9H) ppm. ${ }^{13} \mathrm{C}$ NMR: $\delta 210.6,210.5,139.3,135.3,135.2,132.8,132.7$, $130.7,130.6,128.1,128.0,116.9,115.6,75.0,70.7,63.0,51.8,51.6,45.9,45.7,37.1$, 34.4, 34.3, 25.9. IR (neat): 3517, 3073, 2954, 2875, 1707, 1125, 1115, 1086, 741, 720, $698 \mathrm{~cm}^{-1}$. HRMS: Calcd for $\mathrm{C}_{27} \mathrm{H}_{36} \mathrm{O}_{4} \mathrm{SiNa} 475.2267\left(\mathrm{M}+\mathrm{Na}^{+}\right)$. Found: 475.2275 .

\section{4-(3-Butenoxydiisopropylsilyloxy)-7-hydroxy-8,8-dimethyl-non-8-ene-5-one}

(92a/93a). (Entry 4, table 4). Reaction of 65a (71 mg, $0.26 \mathrm{mmol}$ ) with $\mathrm{Cy}_{2} \mathrm{BOTf}$ gave 192a/93a in $85 \%(79 \mathrm{mg})$, ds $=2: 1$. Spectral data reported for the racemic product. ${ }^{1} \mathrm{H}$ NMR: $\delta 5.84(\mathrm{~m}, 2 \mathrm{H}), 5.12(\mathrm{~m}, 4 \mathrm{H}), 4.83(\mathrm{~m}, 1 \mathrm{H}), 3.78(\mathrm{t}, J=6.6 \mathrm{~Hz}, 2 \mathrm{H}), 2.93(\mathrm{~d}, J=$ $3 \mathrm{~Hz}, 1 \mathrm{H}), 2.78-2.52(\mathrm{~m}, 4 \mathrm{H}), 2.33(\mathrm{dt}, J=13.5,6.6 \mathrm{~Hz}, 2 \mathrm{H}), 1.04(\mathrm{~s}, 6 \mathrm{H}), 1.03(\mathrm{~s}, 6 \mathrm{H})$, 1.00 (m, 2H), 0.92 (s, 9H) ppm. ${ }^{13} \mathrm{C}$ NMR: $\delta 210.8,139.9,135.3,116.5,114.8,114.7$, 74.6, 70.0, 69.9, 62.6, 52.0, 51.8, 46.0, 45.8, 37.2, 34.1, 34.0, 25.6, 17.4, 17.3, 17.1, 17.0, 12.2 ppm. IR (neat): $3500,2946,2868,1707,1092,1094,885,691 \mathrm{~cm}^{-1}$.

\section{(3RS,7R,8Z)-7,11- $O$-diisopropylsilanediyl-3-hydroxy-2,2,8-trimethyl-undec-8-ene-5-} one (92b/93b). (Entry 5, table 4) Reaction of 65b (106 mg, $0.37 \mathrm{mmol}$ ) with (-)-Ipc $\mathrm{ICl}_{2}$ gave $\mathbf{9 2 b} / 93 \mathbf{b}$ in $88 \%(120 \mathrm{mg})$, ds $=1.5: 1$. Spectral data reported for one diastereomer, separated by flash chromatography. ${ }^{1} \mathrm{H}$ NMR: $\delta 5.43(\mathrm{t}, J=8.7 \mathrm{~Hz}, 1 \mathrm{H}), 4.89$ (dd, $J=9.6$, 3.3, Hz, 1H), $3.85(\mathrm{~m}, 2 \mathrm{H}), 3.00-2.48(\mathrm{~m}, 5 \mathrm{H}), 2.22(\mathrm{~m}, 1 \mathrm{H}), 1.75(\mathrm{~s}, 3 \mathrm{H}), 1.02(\mathrm{~m}$, $14 \mathrm{H}), 0.93$ (s, 9H) ppm. ${ }^{13} \mathrm{C}$ NMR: $\delta 211.9,140.5,125.0,74.7,71.2,64.3,49.5,46.4$, $34.3,30.8,25.9,21.2,17.7,17.6,17.5,17.4,12.8,12.5$ ppm. IR (neat): $3479,2954,2868$, 1709, 1114, 1006, 940, 733, $696 \mathrm{~cm}^{-1}$. HRMS: Calcd for $\mathrm{C}_{20} \mathrm{H}_{39} \mathrm{O}_{4} \mathrm{Si} 371.2602\left(\mathrm{M}+\mathrm{H}^{+}\right)$. Found: 371.2612 . 
(3RS,7R,8Z)-7,11- $O$-diisopropylsilanediyl-3-hydroxy-2,2,8-trimethylundec-8-ene-5one (92b/93b). (Entry 6, table 4) Reaction of $\mathbf{6 5 b}$ (30 mg, $0.11 \mathrm{mmol}$ ) with $\mathrm{Cy}_{2} \mathrm{BOTf}$ gave $\mathbf{9 2 b} / \mathbf{9 3 b}$ in $95 \%(37 \mathrm{mg})$, ds $=1.2: 1$. Spectral data matched that reported above.

(3R,7R,8Z)-7,11- $O$-diphenylsilanediyl-3-hydroxy-2,2,8-trimethylundec-8-ene-5-one (92c). (Entry 7, table 4) Reaction of $\mathbf{6 5 c}(100 \mathrm{mg}, 0.28 \mathrm{mmol})$ with (-)- $\mathrm{Ipc}_{2} \mathrm{BCl}$ gave $92 \mathrm{c}$ in $94 \%$ (115 mg), ds = 99>1. ${ }^{1} \mathrm{H}$ NMR: $\delta 7.61(\mathrm{~m}, 4 \mathrm{H}), 7.38(\mathrm{~m}, 6 \mathrm{H}), 5.44(\mathrm{t}, J=9.6 \mathrm{~Hz}$, $1 \mathrm{H}), 4.90$ (dd, $J=9.6,3.3 \mathrm{~Hz}, 1 \mathrm{H}), 4.06(\mathrm{~m}, 2 \mathrm{H}), 3.76(\mathrm{dd}, J=10.5,1.8 \mathrm{~Hz}, 1 \mathrm{H}), 2.99$ (dd, $J=14.9,9.8 \mathrm{~Hz}, 1 \mathrm{H}), 2.91(\mathrm{~m}, 1 \mathrm{H}), 2.76(\mathrm{dd}, J=17.8,1.8 \mathrm{~Hz}, 1 \mathrm{H}), 2.62$ (dd, $J=$ $14.7,3.6 \mathrm{~Hz}, 1 \mathrm{H}), 2.58$ (dd, $J=17.7,10.2 \mathrm{~Hz}, 1 \mathrm{H}), 2.28(\mathrm{~m}, 1 \mathrm{H}), 1.61(\mathrm{~s}, 3 \mathrm{H}), 0.89$ (s, $9 \mathrm{H}) \mathrm{ppm} .{ }^{13} \mathrm{C}$ NMR: $\delta 211.5,140.7,134.8,134.7,133.6,133.3,130.5,130.4,128.1,74.8$, $71.9,64.3,49.5,46.1,34.3,30.9,25.9,21.5$ ppm. IR (neat): 3480, 3070, 2955, 2873, 2247, 1706, 1116, 1073, 909, 730, 716, $699 \mathrm{~cm}^{-1}$. HRMS: Calcd for $\mathrm{C}_{26} \mathrm{H}_{34} \mathrm{O}_{4} \mathrm{SiNa}$ $461.2120\left(\mathrm{M}+\mathrm{Na}^{+}\right)$. Found: 461.2119 .

\section{(3R,7R,8Z)-7,11-O-diphenylsilanediyl-3-hydroxy-2,2,8-trimethylundec-8-ene-5-one} (92c). (Entry 8, table 4). (Unoptimised). Reaction of 65c (127 mg, $0.36 \mathrm{mmol}$ ) with $\mathrm{Cy}_{2}$ BOTf gave $92 \mathrm{c}$ in $68 \%(108 \mathrm{mg}), \mathrm{ds}=99>1$. Spectral data matched that reported above.

(3R,7R,8Z)-7,11- $O$-diphenylsilanediyl-3-hydroxy-2,2-dimethylundec-8ene-5-one (92d). (Entry 9, table 4). (Unoptimised). Reaction of 92d (114 mg, $0.34 \mathrm{mmol}$ ) with (-)$\mathrm{Ipc}_{2} \mathrm{BCl}$ gave 19d in 60\% (86 mg), ds = 99>1. ${ }^{1} \mathrm{H}$ NMR: $\delta 7.64(\mathrm{~m}, 4 \mathrm{H}), 7.40(\mathrm{~m}, 6 \mathrm{H})$, $5.74(\mathrm{~m}, 2 \mathrm{H}), 5.14(\mathrm{~m}, 1 \mathrm{H}), 4.09(\mathrm{~m}, 1 \mathrm{H}), 3.94(\mathrm{~m}, 1 \mathrm{H}), 3.74(\mathrm{~d}, J=10.2, \mathrm{~Hz}, 1 \mathrm{H}), 3.03-$ 2.39 (m, 6H), 0.88 (s, 9H) ppm. ${ }^{13} \mathrm{C}$ NMR: $\delta 211.2,135.0,134.7,133.5,133.4,130.6$, $130.4,129.4,128.2,128.1,74.8,67.6,63.7,51.3,46.1,34.3,31.0,25.9$ ppm. IR (neat): $3509,3019,2959,2873,1709,1125,1070,723 \mathrm{~cm}^{-1}$. HRMS: Calcd. for $\mathrm{C}_{25} \mathrm{H}_{32} \mathrm{O}_{4} \mathrm{SiNa}$ $447.1961\left(\mathrm{M}+\mathrm{Na}^{+}\right)$. Found: 447.1962. 


\subsection{Experimental for chapter 7:}

Representative procedure for the synthesis of the tert-butyldiphenylsiloxy ketones

To a solution of the $\beta$-hydroxyketone $(1 \mathrm{mmol})$ in DMF $(1.5 \mathrm{~mL})$ was added tertbutyldiphenylsilylchloride $(1.1 \mathrm{mmol})$ and imidazole $(2 \mathrm{mmol})$. After stirring at room temperature for $24 \mathrm{~h}$ the solution was quenched with water then extracted twice with $\mathrm{Et}_{2} \mathrm{O}$. The organic extracts were washed with brine, dried over $\mathrm{MgSO}_{4}$, filtered and the solvents removed under reduced pressure. The residual oil was purified by flash chromatography, (50:1 hexanes:EtOAc) to give the silylated adducts as colourless oils.

(4R)-4-tert-Butyldiphenylsiloxy-5-methylhexan-2-one (95a). 4-hydroxy-5-methylhexane-2-one (94a) (646 mg, $5.0 \mathrm{mmol}$ ) gave 95a (1.32 g, 72\% yield). ${ }^{1} \mathrm{H}$ NMR: $\delta 7.70$ (m, 4H), $7.42(\mathrm{~m}, 6 \mathrm{H}), 4.18(\mathrm{~m}, 1 \mathrm{H}), 2.56(\mathrm{dd}, J=15.9,6.6 \mathrm{~Hz}, 1 \mathrm{H}), 2.42(\mathrm{dd}, J=15.9$, $5.4 \mathrm{~Hz}, 1 \mathrm{H}), 1.88(\mathrm{~s}, 3 \mathrm{H}), 1.72(\mathrm{~m}, 1 \mathrm{H}), 1.06(\mathrm{~s}, 9 \mathrm{H}), 0.94$ (d, J = 6.6 Hz), 0.81 (d, J = 6.9 $\mathrm{Hz})$ ppm. ${ }^{13} \mathrm{C}$ NMR: $\delta 207.6,136.3,1362,134.5,134.3,129.9,127.8,127.7,73.9,47.8$, $33.7,30.8,27.3,19.8,18.0,17.5$ ppm. IR (neat): 3072, 2960, 2932, 2857, 1715, 1660 , 1110, 1068, 740, 703 $\mathrm{cm}^{-1}$. HRMS: Calcd for $\mathrm{C}_{23} \mathrm{H}_{33} \mathrm{O}_{2} \mathrm{Si} 369.2305\left(\mathrm{M}+\mathrm{H}^{+}\right)$. Found: 369.2245 .

(R)-4-tert-Butyldiphenylsiloxy-5-methylhex-5-en-2-one (95c). 4-hydroxy-5-methylhex5-ene-2-one (94c) (546 mg, $4.2 \mathrm{mmol})$ gave $1.15 \mathrm{~g}$ (75\%) of 95c. ${ }^{1} \mathrm{H}$ NMR: $\delta 7.65$ (m, $4 \mathrm{H}), 7.38(\mathrm{~m}, 6 \mathrm{H}), 4.78(\mathrm{~s}, 1 \mathrm{H}), 4.72(\mathrm{~s}, 1 \mathrm{H}), 4.59(\mathrm{t}, J=6 \mathrm{~Hz}, 1 \mathrm{H}), 2.61$ (dd, $J=14.7$, $6.0 \mathrm{~Hz}, 1 \mathrm{H}), 2.52(\mathrm{dd}, J=14.7,6.6 \mathrm{~Hz}, 1 \mathrm{H}), 2.10(\mathrm{~s}, 3 \mathrm{H}), 1.68(\mathrm{~s}, 3 \mathrm{H}), 1.06(\mathrm{~s}, 9 \mathrm{H}) \mathrm{ppm}$. ${ }^{13}$ C NMR: $\delta 206.8,145.8,136.2,134.1,133.7,130.0,128.0,127.8,127.7,112.6,74.1$, 50.8, 31.1, 27.2, 19.6, 17.4 ppm. IR (neat): 3072, 2931, 2857, 1713, 1427, 1360, 1162, 1106, 1063, 739, $700 \mathrm{~cm}^{-1}$. HRMS: Calcd. for $\mathrm{C}_{23} \mathrm{H}_{30} \mathrm{O}_{2} \mathrm{SiNa} 389.1922\left(\mathrm{M}+\mathrm{Na}^{+}\right)$. Found: 389.1907. 


\section{Representative procedure for aldol reactions}

All aldol reactions reported in this section were conducted in the following manner: To a stirred solution of $\mathrm{Cy}_{2} \mathrm{BCl}(1.5 \mathrm{mmol})$ in anhydrous $\mathrm{Et}_{2} \mathrm{O}(4 \mathrm{~mL})$ at $0{ }^{\circ} \mathrm{C}$ was slowly added $\mathrm{Et}_{3} \mathrm{~N}(1.7 \mathrm{mmol})$. A solution of the ketone $(1 \mathrm{mmol})$ in $\mathrm{Et}_{2} \mathrm{O}(2 \mathrm{~mL})$ was then added via cannula. The reaction mixture was stirred at $0{ }^{\circ} \mathrm{C}$ for $30 \mathrm{~min}$ before being cooled to $-78^{\circ} \mathrm{C}$ at which point the aldehyde $(1.5 \mathrm{mmol})$ was slowly added dropwise. The solution was then stirred at $-78^{\circ} \mathrm{C}$ for $5-6 \mathrm{~h}$, before being quenched at $-78^{\circ} \mathrm{C}$ by the addition of $\mathrm{pH} 7$ buffer solution, methanol and hydrogen peroxide (30\% solution). The solution was stirred at room temperature for $2.5 \mathrm{~h}$ then extracted twice with $\mathrm{Et}_{2} \mathrm{O}$. The organic extracts were washed with water, brine, dried over $\mathrm{MgSO}_{4}$, filtered and concentrated under reduced pressure. Purification of the crude material by gradient flash chromatography (50:1 to 10:1 hexanes:EtOAc) gave the aldol adducts as colourless oils.

(3RS,7R)-7-tert-Butyldiphenylsiloxy-3-hydroxy-2,2,8-trimethylnonan-5-one (96a). The aldol reaction of $95 \mathrm{a}(201 \mathrm{mg}, 0.52 \mathrm{mmol})$ with pivaldehyde gave $96 \mathbf{a}$ (201 $\mathrm{mg}, 88 \%$ yield), $\mathrm{ds}=2: 1$. Spectral data reported for one diastereomer, separated by flash chromatography. 'H NMR: $\delta 7.74$ (dd, $J=7.2,1.2 \mathrm{~Hz}, 2 \mathrm{H}), 7.68(\mathrm{dd}, J=7.5,1.2 \mathrm{~Hz}, 2 \mathrm{H})$, $7.42(\mathrm{~m}, 6 \mathrm{H}), 4.20(\mathrm{~m}, 1 \mathrm{H}), 3.52(\mathrm{dd}, J=9.0,2.1 \mathrm{~Hz}, 1 \mathrm{H}), 2.61(\mathrm{dd}, J=16.2,6.6 \mathrm{~Hz}$, $1 \mathrm{H}), 2.43$ (dd, $J=16.2,4.8 \mathrm{~Hz}, 1 \mathrm{H}), 2.28(\mathrm{~m}, 2 \mathrm{H}), 1.73(\mathrm{~m}, 1 \mathrm{H}), 1.06(\mathrm{~s}, 9 \mathrm{H}), 0.95$ (d, $J$ $=6.3 \mathrm{~Hz}, 3 \mathrm{H}), 0.86(\mathrm{bs}, 6 \mathrm{H}), 0.81(\mathrm{~d}, \mathrm{~J}=6.6 \mathrm{~Hz}) \mathrm{ppm} .{ }^{13} \mathrm{C} \mathrm{NMR}: \delta 211.3,136.3,136.2$, $134.6,134.1,129.9,127.8,74.7,73.6,47.7,45.2,34.2,33.7,27.3,25.9,19.7,17.9,17.6$ ppm. IR (neat): 3502, 3072, 2957, 2858, 1709, 1110, 1057, 823, $702 \mathrm{~cm}^{-1}$. HRMS: Calcd for $\mathrm{C}_{28} \mathrm{H}_{42} \mathrm{O}_{3} \mathrm{SiNa} 477.2854\left(\mathrm{M}+\mathrm{Na}^{+}\right)$. Found: 477.2796 .

(3RS,7R)-7-tert-Butyldiphenylsiloxy-3-hydroxy-2,8-dimethylnonan-5-one (96b). The aldol reaction of $95 \mathbf{a}(119 \mathrm{mg}, 0.32 \mathrm{mmol})$ and isobutylaldehyde gave $\mathbf{9 6 \mathbf { b }}(125 \mathrm{mg}, 86 \%$ yield), ds $=2.5: 1$. Spectral data reported for one diastereomer (separated by flash chromatography). ' $\mathrm{H}$ NMR: $\delta 7.69$ (dd, $J=7.8,1.5 \mathrm{~Hz}, 2 \mathrm{H}), 7.63$ (dd, $J=7.8,1.5 \mathrm{~Hz}$, 
2H), $7.37(\mathrm{~m}, 6 \mathrm{H}), 4.16(\mathrm{~m}, 1 \mathrm{H}), 3.55(\mathrm{dt}, J=11.7,6.0 \mathrm{~Hz}, 1 \mathrm{H}), 2.79$ (bs, $1 \mathrm{H}), 2.55$ $2.24(\mathrm{~m}, 4 \mathrm{H}), 1.68(\mathrm{~m}, 1 \mathrm{H}), 1.54(\mathrm{~m}, 1 \mathrm{H}), 1.02(\mathrm{~s}, 9 \mathrm{H}), 0.90(\mathrm{~d}, J=6.9 \mathrm{~Hz}, 3 \mathrm{H}), 0.84$ (d, $J=6.9 \mathrm{~Hz}, 3 \mathrm{H}), 0.81(\mathrm{~d}, J=6.9 \mathrm{~Hz}, 3 \mathrm{H}), 0.77(\mathrm{~d}, \mathrm{~J}=6.9 \mathrm{~Hz}) \mathrm{ppm} .{ }^{13} \mathrm{C}$ NMR: $\delta 211.2$, $136.3,136.2$, 134.6, 134.1, 130.0, 129.8, 127.8, 127.7, 73.6, 72.0, 47.6, 47.1, 33.7, 33.0, 27.3, 19.7, 18.6, 18.0, 17.9, 17.6 ppm. IR (neat): 3478, 3072, 2929, 2932, 2859, 1707, 1109, 1006, 760, 701 $\mathrm{cm}^{-1}$. HRMS: Calcd for $\mathrm{C}_{27} \mathrm{H}_{40} \mathrm{O}_{3} \mathrm{SiNa} 463.2653\left(\mathrm{M}+\mathrm{Na}^{+}\right)$. Found: 463.2638 .

\section{(3RS,7R)-7-tert-Butyldiphenylsiloxy-2,8-dimethyl-3-hydroxynona-1-8-diene-5-one}

(96c). The aldol reaction of $95 \mathbf{c}(453 \mathrm{mg}, 1.23 \mathrm{mmol})$ with methacrolein gave $96 \mathbf{c}$ (505 mg, $89 \%$ yield), ds $=1.2: 1$. Spectral data reported for the combined diastereomers. ${ }^{1} \mathrm{H}$ NMR: $\delta 7.67(\mathrm{~m}, 4 \mathrm{H}), 7.40(\mathrm{~m}, 6 \mathrm{H}), 4.95$ (s, 1H), $4.83(\mathrm{bs}, 2 \mathrm{H}), 4.77(\mathrm{~s}, 1 \mathrm{H}), 4.61(\mathrm{~m}$, $1 \mathrm{H}), 4.32$ (m, 1H), 3.90 (bs, 1H), $2.73-2.2 .40(\mathrm{~m}, 4 \mathrm{H}), 1.70(\mathrm{~s}, 3 \mathrm{H}), 1.68(\mathrm{~s}, 3 \mathrm{H}), 1.07$ (s, 9H). ${ }^{13} \mathrm{C}$ NMR: $\delta$ 209.5, 145.7, 136.2, 136.1, 134.1, 133.5, 130.1, 130.0, 127.9, 127.8, $112.7,111.4,73.8,73.7,70.9,50.5,49.0,48.8,27.2,19.6,18.6,18.5,17.5$ ppm. IR (neat): $3450,3073,2932,2858,1709,1111,1072,822,702,612 \mathrm{~cm}^{-1}$. HRMS: Calcd for $\mathrm{C}_{27} \mathrm{H}_{37} \mathrm{O}_{3} \mathrm{Si}: 437.2528\left(\mathrm{M}+\mathrm{H}^{+}\right)$. Found: 437.2507.

\section{Representative procedure for the synthesis of the dimethylphenylsiloxy ketones}

To a solution of the $\beta$-hydroxyketone $(1 \mathrm{mmol})$ in DMF $(1.5 \mathrm{~mL})$ was added dimethylphenylsilylchloride $(1.1 \mathrm{mmol})$ and imidazole $(2.2 \mathrm{mmol})$. After stirring at room temperature for $24 \mathrm{~h}$, the solution was quenched with water then extracted twice with $\mathrm{Et}_{2} \mathrm{O}$. The organic extracts were washed with brine, dried over $\mathrm{MgSO}_{4}$, filtered and the solvents removed under reduced pressure. The residual oil was purified by flash chromatography (20:1 hexanes:EtOAc) to give the silylated adducts as colourless oils. 
(R)-4-Dimethylphenylsiloxy-5-methyl-hexan-2-one (97a). Silylation of $(R)$-4-hydroxy5-methyl-hexan-2-one (94a) (450 mg, $3.48 \mathrm{mmol})$ gave $97 \mathbf{a}(682 \mathrm{mg}, 74 \%$ yield) after purification by flash chromatography. ${ }^{1} \mathrm{H}$ NMR: $\delta 7.57(\mathrm{~m}, 2 \mathrm{H}), 7.34(\mathrm{~m}, 3 \mathrm{H}), 4.06$ (ddd, $J=12.3,8.1,3.9 \mathrm{~Hz}, 1 \mathrm{H}), 2.60(\mathrm{~d}, J=15.6,8.1 \mathrm{~Hz}, 1 \mathrm{H}), 2.36(\mathrm{dd}, J=15.6,3.9 \mathrm{~Hz}, 1 \mathrm{H})$, 2.14, s (3H), $1.66(\mathrm{~m}, 1 \mathrm{H}), 0.85(\mathrm{~d}, J=6.9 \mathrm{~Hz}, 3 \mathrm{H}), 0.82$ (d, $J=6.9 \mathrm{~Hz}, 3 \mathrm{H}), 0.37$ (s, $3 \mathrm{H}), 0.36$ (s, 3H) ppm. ${ }^{13} \mathrm{C}$ NMR: $\delta 207.9,138.2,133.3,129.3,127.6,73.5,47.3,33.6$, $31.2,17.7,17.5,-1.3,-1.4$ ppm. IR (neat): 3071, 2960, 1717, 1357, 1250, 1117, 1079, $1051,829,782,699 \mathrm{~cm}^{-1}$.

(4R)-4-Dimethylphenylsiloxy-5-methylhex-5-en-2-one (97b). Silylation of (4R)-4hydroxy-5-methylhex-5-en-2-one (94a) (250 mg, $1.95 \mathrm{mmol}$ ) gave 97b (404 mg, 72\% yield) as a colourless oil after purification by flash chromatography. ${ }^{1} \mathrm{H}$ NMR: $\delta 7.55$ (m, 2H), $7.36(\mathrm{~m}, 3 \mathrm{H}), 4.90$ (s, 1H), 4.77 (s, 1H), 4.57 (dd, $J=8.7,3.9 \mathrm{~Hz}, 1 \mathrm{H}), 2.75$ (dd, $J=$ 14.7, $8.7 \mathrm{~Hz}, 1 \mathrm{H}), 2.42(\mathrm{dd}, J=14.7,3.9 \mathrm{~Hz}, 1 \mathrm{H}), 2.10$ (s, 3H), 1.67 (s, 1H), 0.36 (s, 3H), 0.35 (s, 3H) ppm. ${ }^{13} \mathrm{C}$ NMR: $\delta 206.9,146.1,137.5,133.3,129.4,127.5,111.4,73.2,50.0$, 31.1, 17.2, -1.5, -1.8 ppm; IR (neat): 3072, 2958, 1714, 1251, 1162, 1070, 830, 814, 783, $699 \mathrm{~cm}^{-1}$. HRMS: Cald for $\mathrm{C}_{15} \mathrm{H}_{22} \mathrm{O}_{2} \mathrm{NSi} 280.1719\left(\mathrm{M}+\mathrm{NH}_{4}^{+}\right)$. Found: 280.1727 .

(3RS,7R)-3-Hydroxy-2,2,8-trimethyl-7-dimethylphenylsiloxy-nonane-5-one (98a). The aldol reaction of ketone $97 \mathrm{a}(100 \mathrm{mg}, 0.38 \mathrm{mmol})$ with pivaldehyde gave the corresponding aldol adduct $98 \mathbf{a}(106 \mathrm{mg}, 80 \%$ yield), ds: $1: 1.1$. Spectral data reported for one diastereomer (separated by flash chromatography). ${ }^{1} \mathrm{H}$ NMR: $\delta 7.58(\mathrm{~m}, 2 \mathrm{H}), 7.38$ $(\mathrm{m}, 3 \mathrm{H}), 4.10$ (ddd, $J=9.0,8.4,3.6 \mathrm{~Hz}, 1 \mathrm{H}), 3.63(\mathrm{dd}, J=10.4,1.9 \mathrm{~Hz}, 1 \mathrm{H}), 2.71-2.31$ $(\mathrm{m}, 4 \mathrm{H}), 1.68(\mathrm{~m}, 1 \mathrm{H}), 0.88(\mathrm{~s}, 9 \mathrm{H}), 0.84(\mathrm{~m}, 6 \mathrm{H}), 0.39(\mathrm{~s}, 6 \mathrm{H}) \mathrm{ppm} .{ }^{13} \mathrm{C}$ NMR: $\delta 212.3$, $138.2,133.5,130.0,127.7,74.9,73.7,47.1,45.8,34.1,33.6,25.6,17.9,17.5,-1.2,-1.4$ ppm. IR (neat): $3500,2959,2905,2873,1709,1252,1117,1056,829,785,744,700 \mathrm{~cm}^{-1}$. 
(3RS,7R)-3-Hydroxy-2,8-dimethyl-7-dimethylphenylsiloxynona-1,8-dien-5-one (98b). The aldol reaction of ketone $\mathbf{9 7 b}(100 \mathrm{mg}, 0.38 \mathrm{mmol})$ with methacrolein gave the corresponding aldol adduct $\mathbf{9 8 b}$ (104 $\mathrm{mg}, 82 \%$ yield), ds: $1: 1.1$. Spectral data reported for the combined diastereomers. ${ }^{1} \mathrm{H}$ NMR: $\delta 7.59(\mathrm{~m}, 2 \mathrm{H}), 7.40(\mathrm{~m}, 3 \mathrm{H}), 5.00(\mathrm{~d}, J=0.6 \mathrm{~Hz}$, $1 \mathrm{H}), 4.94(\mathrm{~s}, 1 \mathrm{H}), 4.86(\mathrm{~s}, 1 \mathrm{H}), 4.81(\mathrm{t}, J=1.2 \mathrm{~Hz}, 1 \mathrm{H}), 4.61(\mathrm{~m}, 1 \mathrm{H}), 4.45(\mathrm{t}, J=6.3 \mathrm{~Hz}$, $1 \mathrm{H}), 2.85-2.41$ (m, 4H), 1.72 (d, $J=3.6 \mathrm{~Hz}, 3 \mathrm{H}), 1.69$ (s, 3H), 0.40 (s, 6H), 0.39 (s, $3 \mathrm{H}), 0.38$ (s, 3H) ppm; ${ }^{13} \mathrm{C}$ NMR: $\delta 210.4,210.3,146.4,146.3,145.8,133.8,133.3$, $130.0,129.9,128.1,128.0,112.2,112.1,111.4,73.7,71.3,71.0,50.3,50.2,49.7,49.6$, 18.6, 18.5, -1.0, - 1.1, -1.5 ppm. IR (neat): 3420, 3072, 2958, 1708, 1251, 1117, 1071, 829, 784, $700 \mathrm{~cm}^{-1}$. HRMS: Cald for $\mathrm{C}_{19} \mathrm{H}_{32} \mathrm{NO}_{3} \mathrm{Si} 350.2135\left(\mathrm{M}+\mathrm{NH}_{4}^{+}\right)$. Found: 350.2146 .

(R)-4-Benzyloxy-5-methyl-hexan-2-one (99). To a solution of $(R)$-4-hydroxy-5-methylhexane-2-one ( $240 \mathrm{mg}, 1.86 \mathrm{mmol})$ in $6 \mathrm{~mL}$ of $\mathrm{CH}_{2} \mathrm{Cl}_{2}$ :cyclohexane $(2: 1)$ cooled to $0{ }^{\circ} \mathrm{C}$ was added benzyl trichloroacetimidate (79) $(345 \mu \mathrm{l}, 1.86 \mathrm{mmol})$ followed by freshly distilled trifluoromethanesulfonic acid $(33 \mu \mathrm{l}, 0.37 \mathrm{mmol})$ dropwise. The appearance of a cream precipitate was immediately observed and the resulting mixture stirred at room temperature for $30 \mathrm{~min}$ before being quenched with water $(5 \mathrm{~mL})$. The solution was then extracted twice with hexanes and the organic extracts washed with satd. aq. $\mathrm{NaHCO}_{3}$, brine, dried over $\mathrm{MgSO}_{4}$, filtered and concentrated under reduced pressure. Purification of the crude material by flash chromatography (20:1 hexanes:EtOAc) gave 99 (216 mg, $60 \%$ ) as a colourless oil. ${ }^{1} \mathrm{H}$ NMR: $\delta 7.33(\mathrm{~m}, 5 \mathrm{H}), 4.57(\mathrm{~d}, J=11.4 \mathrm{~Hz}, 1 \mathrm{H}), 4.51(\mathrm{~d}, J=$ $11.4 \mathrm{~Hz}, 1 \mathrm{H}), 3.83(\mathrm{~m}, 1 \mathrm{H}), 2.71(\mathrm{~d}, J=15.9,8.4 \mathrm{~Hz}, 1 \mathrm{H}), 4.46(\mathrm{~d}, J=15.9,3.6 \mathrm{~Hz}, 1 \mathrm{H})$, $2.16(\mathrm{~s}, 3 \mathrm{H}), 1.98(\mathrm{~m}, 1 \mathrm{H}), 0.96(\mathrm{~d}, J=6.9 \mathrm{~Hz}, 6 \mathrm{H}) \mathrm{ppm} .{ }^{13} \mathrm{C}$ NMR: $\delta 207.8,138.4,128.5$, $128.0,127.4,127.3,127.2,79.9,71.8,44.6,30.9,30.6,17.9,17.2$ ppm. IR (neat): 3032 , 2960, 2874, 1712, 1355, 1070, 733, $697 \mathrm{~cm}^{-1}$. HRMS: Cald for $\mathrm{C}_{14} \mathrm{H}_{21} \mathrm{O}_{2} 221.1548(\mathrm{M}+$ $\mathrm{H}^{+}$). Found: 221.1536 . 
(3RS,7R)-7-Benzyloxy-3-hydroxy-2,2,8-trimethylnonan-5-one (100). The aldol reaction of ketone $99(240 \mathrm{mg}, 1.09 \mathrm{mmol})$ with pivaldehyde gave the corresponding aldol adduct 100 (246 mg, 74\% yield), ds: 3:1. Spectral data reported for the combined diastereomers). ${ }^{1} \mathrm{H}$ NMR: $\delta 7.33$ (m, 5H), 4.53 (m, 2H), 3.85 (m, 1H), 3.73 (dd, $J=10.2$, $1.8 \mathrm{~Hz}, 1 \mathrm{H}), 2.79-2.42(\mathrm{~m}, 4 \mathrm{H}), 1.98(\mathrm{~m}, 1 \mathrm{H}), 0.95$ (d, $J=6.9 \mathrm{~Hz}, 6 \mathrm{H}), 0.89$ (s, 9H) ppm. ${ }^{13} \mathrm{C}$ NMR: $\delta 212.3,138.8,129.2,128.6,128.3,128.2,128.0,127.9,127.8,80.7$, $80.5,74.9,72.5,72.4,46.2,45.5,45.2,45.1$, 34.4, 31.1, 25.9, 18.6, 18.5, 17.7, 17.6 ppm. IR (neat): 3500, 3032, 2959, 2871, 1706, 1067, 734, $697 \mathrm{~cm}^{-1}$. HRMS: Cald for $\mathrm{C}_{19} \mathrm{H}_{34} \mathrm{NO}_{3} 324.2529\left(\mathrm{M}+\mathrm{NH}_{4}^{+}\right)$Found:324.2533.

4-(But-1-ene-4-yloxy)-but-3-ene-2-one (105). A $50 \mathrm{~mL}$ round bottom flask equipped with stir bar, $8 \mathrm{~cm}$ Vigreux column and distillation head was charged with but-3-enol (1.07 mL, $12.49 \mathrm{mmol}$ ), 4-methoxybut-3-en-2-one (1.53 mL, $14.98 \mathrm{mmol}$ ), pyridinium $p$ toluenesulfonate $(31 \mathrm{mg}, 0.12 \mathrm{mmol})$ and toluene $(25 \mathrm{~mL})$. The reaction mixture was heated for $4 \mathrm{~h}$ at a gentle reflux, allowing for a slow distillation of the methanol-toluene mixture. After cooling the solution to room temperature, the solvent was removed under reduced pressure to give a red/brown oil. The crude material was purified by flash chromatography (10:1 hexanes:EtOAc) to give $105(1.15 \mathrm{~g}, 66 \%)$ as an orange oil. ${ }^{1} \mathrm{H}$ NMR: $\delta 7.50(\mathrm{~d}, J=12.6 \mathrm{~Hz}, 1 \mathrm{H}), 5.76(\mathrm{~m}, 1 \mathrm{H}), 5.55(\mathrm{~d}, J=12.6 \mathrm{~Hz}, 1 \mathrm{H}), 5.09(\mathrm{~m}, 2 \mathrm{H})$, $3.88(\mathrm{t}, J=6.6 \mathrm{~Hz}, 1 \mathrm{H}), 2.43(\mathrm{dt}, J=13.2,6.6, \mathrm{~Hz}, 2 \mathrm{H}), 2.14(\mathrm{~s}, 3 \mathrm{H}) \mathrm{ppm} .{ }^{13} \mathrm{C}$ NMR: $\delta$ $197.2,162.3,133.2,117.6,107.1,70.0,33.0,27.6$ ppm. IR (neat): 3079, 2947, 1618, $1591,1202,1147,957,920 \mathrm{~cm}^{-1}$.

\section{1-[(2S,4R,6S)-(6-Allyl-4-(tert-butyldimethylsiloxy)tetrahydro-pyran-2-yl]-propan-2-}

one (109). To a solution of alcohol $108(200 \mathrm{mg}, 1.01 \mathrm{mmol})$ in DMF (3 mL)was added tert-butyldimethylsilylchloride (167 mg, $1.11 \mathrm{mmol}$ ) and imidazole (137 mg, $2.02 \mathrm{mmol})$. After stirring at room temperature for $24 \mathrm{~h}$, the solution was quenched with water and 
extracted twice with $\mathrm{Et}_{2} \mathrm{O}$. The organic extracts were then washed with brine, dried over $\mathrm{MgSO}_{4}$, filtered and the solvents removed under reduced pressure. The residual oil was purified by gradient flash chromatography (hexanes:20:1; hexanxes:EtOAc) to give $\mathbf{1 0 9}$ (300 mg, 95\%) as a colourless oil. ${ }^{1} \mathrm{H}$ NMR: $\delta 5.07(\mathrm{~m}, 1 \mathrm{H}), 5.03(\mathrm{~m}, 2 \mathrm{H}), 3.75(\mathrm{~m}, 2 \mathrm{H})$, 3.35 (m, 1H), 2.71 (dd, $J=15.6,7.8 \mathrm{~Hz}, 1 \mathrm{H}), 2.41$ (dd, $J=15.6,4.8 \mathrm{~Hz}, 1 \mathrm{H}), 2.26$ (m, $1 \mathrm{H}), 2.17(\mathrm{~s}, 3 \mathrm{H}), 2.16(\mathrm{~m}, 1 \mathrm{H}), 1.80(\mathrm{~m}, 2 \mathrm{H}), 1.20(\mathrm{~m}, 2 \mathrm{H}), 0.89(\mathrm{~s}, 9 \mathrm{H}), 0.05(\mathrm{~s}, 6 \mathrm{H})$ ppm. ${ }^{13} \mathrm{C}$ NMR: $\delta 207.7,134.9,117.0,75.4,72.3,68.6,49.9,41.6,41.1,40.6,31.4,26.0$, 18.3, -4.4 ppm. IR (neat): 2929, 2856, 1716, 1251, 1124, 1068, 835, $774 \mathrm{~cm}^{-1}$. HRMS: Cald for $\mathrm{C}_{17} \mathrm{H}_{33} \mathrm{O}_{3} \mathrm{Si} 313.2205\left(\mathrm{M}+\mathrm{H}^{+}\right)$. Found: 313.2194 .

(4R)-1-[(2S,4R,6S)-(6-Allyl-4-(tert-butyldimethylsilyloxy)tetrahydro-pyran-2-yl]-4hydroxy-5,5-dimethylhexan-2-one (110). Reaction of ketone 109 (287 mg, $0.92 \mathrm{mmol}$ ) with pivaldehyde gave the corresponding aldol adduct $110(296 \mathrm{mg}, 81 \%)$ as a colourless oil; d.s: >95:5. ${ }^{1} \mathrm{H}$ NMR: $\delta 5.74(\mathrm{~m}, 1 \mathrm{H}), 5.02(\mathrm{~m}, 2 \mathrm{H}), 3.37(\mathrm{~m}, 3 \mathrm{H}), 3.32(\mathrm{~m}, 1 \mathrm{H}), 2.95$ $(\mathrm{d}, J=3 \mathrm{~Hz}, 1 \mathrm{H}), 2.73(\mathrm{dd}, J=15.0,8.4 \mathrm{~Hz}, 1 \mathrm{H}), 2.65(\mathrm{~m}, 1 \mathrm{H}), 2.46(\mathrm{dd}, J=17.1,10.5$, $\mathrm{Hz}, 1 \mathrm{H}), 2.36$ (dd, $J=15.0,3.91 \mathrm{H}), 2.22(\mathrm{~m}, 1 \mathrm{H}), 2.14(\mathrm{~m}, 1 \mathrm{H}), 1.77(\mathrm{~m}, 2 \mathrm{H}), 1.19(\mathrm{~m}$, $2 \mathrm{H}), 0.88(\mathrm{~s}, 9 \mathrm{H}), 0.85(\mathrm{~s}, 9 \mathrm{H}), 0.03(\mathrm{~s}, 6 \mathrm{H}) \mathrm{ppm} .{ }^{13} \mathrm{C}$ NMR: $\delta 211.2,134.4,116.9,75.1$, $74.5,72.3,68.3,49.2,46.1,41.3,40.7,40.1,34.1,25.7,25.6,18.0,-4.7$ ppm. IR (neat): $3478,2952,2857,1708,1643,1071,835,774 \mathrm{~cm}^{-1}$. HRMS: Cald for $\mathrm{C}_{22} \mathrm{H}_{43} \mathrm{O}_{4} \mathrm{Si}$ $399.2928\left(\mathrm{M}+\mathrm{H}^{+}\right)$Found: 399.2925. 


\section{Appendix}

\section{Progress towards the synthesis of peloruside A}

The unique biological activity of peloruside $\mathrm{A}$ has resulted in this compound being a particularly attractive target for many synthetic chemists. Accordingly, in the past few years, several publications regarding the total or partial synthesis of peloruside $A$ have appeared in the literature. Provided below is a review of those publications that appeared prior to the submission of this thesis.

Paterson, Di Francesco and Kühn ${ }^{206}$ reported the first partial synthesis of peloruside A in January 2003, and of the strategies reported thus far, this partial synthesis most closely resembles the strategy pursued by Stocker, Teesdale-Spittle and Hoberg. ${ }^{193}$ The retrosynthetic disconnects for Paterson's synthesis of peloruside A are depicted in scheme 96. Here peloruside A has been divided into three key segments; the C-12 to C-24 fragment 113, the C-7 to C-11 fragment 114 and the C-1 to C-6 fragment $\mathbf{1 1 5}$.

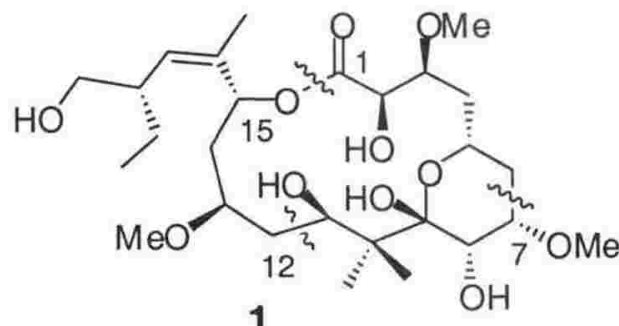

1

$$
\Downarrow
$$

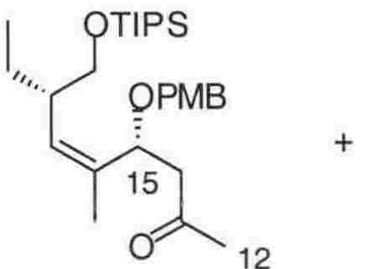

113

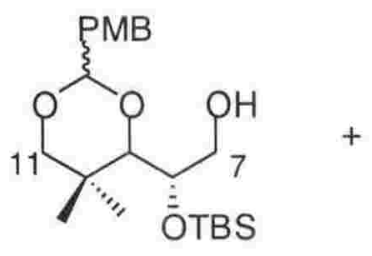

114

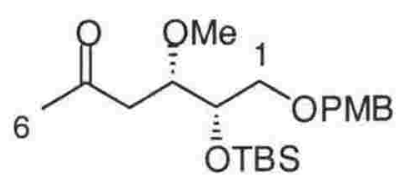

115

Scheme 96: Paterson, Di Francesco and Kühn's retrosynthesis of peloruside A 
The similarity between Paterson's strategy for the synthesis of the C-12 to C-24 fragment of peloruside A and the strategy pursued by Stocker et. al. for the synthesis of the similar carbon framework is most noteworthy. These two fragments are reviewed in figure 36 .

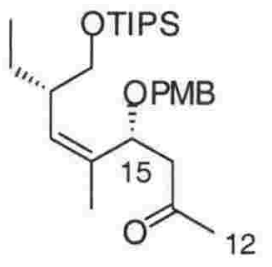

113

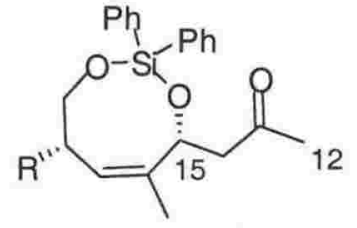

22a $R=E t$ $22 b R=H$

Figure 36: The C-12 to C-24 fragments for Paterson's and Stocker's synthesis of peloruside $\mathrm{A}$

Paterson's synthesis for $\mathbf{1 1 3}$ commences with the $(S)$-lactate-derived ketone $\mathbf{1 1 4}$ - itself synthesised in three steps in a $64 \%$ yield, scheme 97 . A ten step synthesis is required for the formation of $\mathbf{1 1 3}$ and although the individual yields for each of the ten reactions are excellent, due to the linear nature of this strategy, $\mathbf{1 1 3}$ is synthesised in an overall yield of $18 \%$. In contrast, $22 \mathbf{b}$ is synthesised in four linear steps with an overall yield of $33 \%$. Paterson subsequently utilizes boron mediated 1,5-anti aldol methodology to achieve the desired 1,5 anti- relationship between C-15 and C-11. However, in contrast to the synthesis developed by Stocker et. al., whereby the cyclic silyl tether of $\mathbf{2 2}$ induces 1,5anti induction, Paterson relied upon the more highly precedented PMB protecting group at the B-position of $\mathbf{1 1 3}$ for diastereoselective induction.

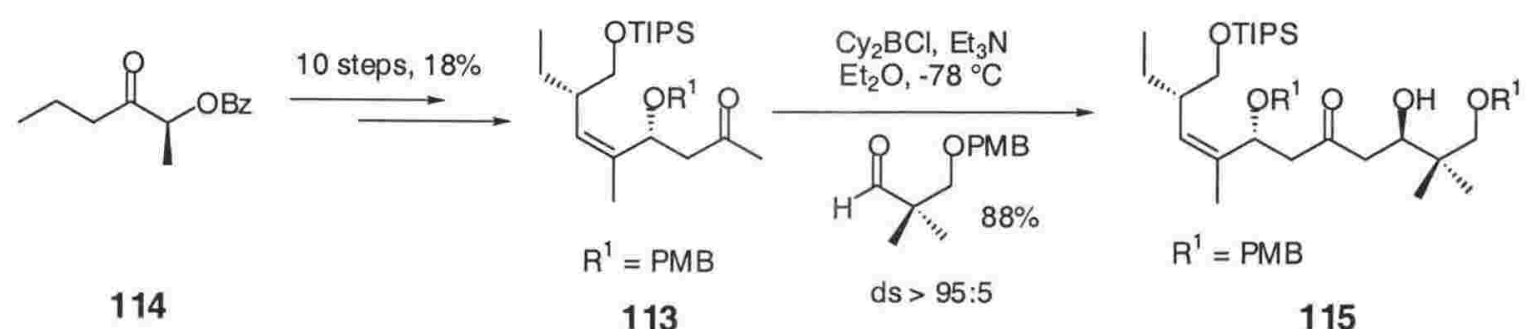

Scheme 97: Paterson, Di Francesco and Kühn's synthesis and aldol studies for the C-12 to $\mathrm{C}-24$ fragment of peloruside $\mathrm{A}$ 
Paterson's synthesis of the C-12 to C-7 fragment of peloruside A starts with neopentylglycol 116. Glycal 116 is subjected to mono benzylation, a Swern oxidation, a Horner-Wadsworth Evans homologation and a Sharpless dihydroxylation, to give key intermediate 117. Perhaps the most interesting feature of this strategy however, was the subsequent DDQ mediated oxidative cyclization of 117 that resulted in an unexpected mixture of PMP acetals 118 and 119. Fortunately though, equilibration of this mixture of acetals with CSA afforded solely the desired, and thermodynamically more stable, sixmembered cyclic acetal 119.

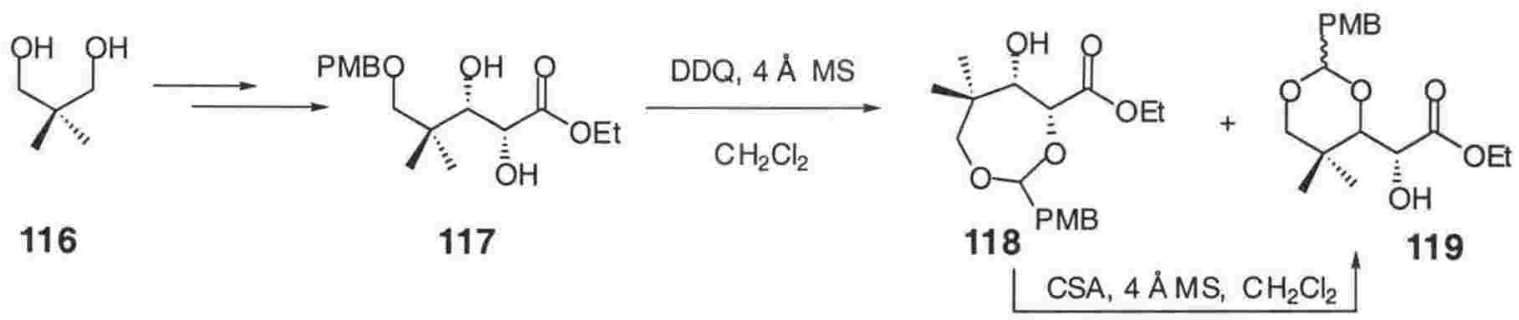

Scheme 98: Paterson, Di Francesco and Kühn's synthesis of the C-11 to C-7 fragment of peloruside $A$

The protection, reduction and oxidation of $\mathbf{1 1 9}$ gave the key retrosynthetic fragment $\mathbf{1 2 0}$, which was subsequently subjected to a variety of aldol reactions, with acetone as a model substrate, in order to ascertain which conditions would favour formation of the desired anti diastereomer 121a. (+)- $\mathrm{Ipc}_{2} \mathrm{BCl}$ was reported to give the highest, although still rather modest, 3:1 ratio of diastereomers, scheme 99.<smiles>CC(C)(C)[C@H]1OCC(C)(C)[C@H]([C@@H]([18OH])C=O)O1</smiles>

120

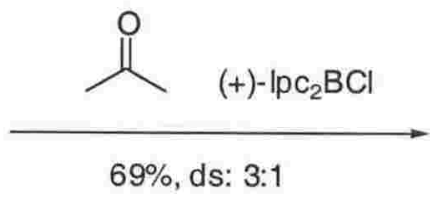

$69 \%$, ds: $3: 1$

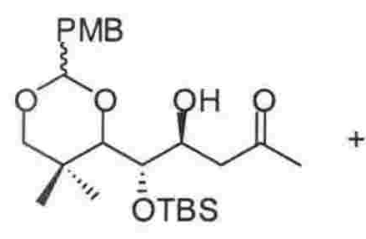

$121 a$<smiles>CC(=O)C[C@H](O)[C@H]([SeH])[C@H]1O[C@H]([13CH3])OCC1(C)C</smiles>

121b

Scheme 99: Model aldol studies with key intermediate 120. 
Finally, Paterson's synthesis of the C-6 to C-1 fragment of peloruside A commenced with methylacetoacetate $\mathbf{1 2 2}$, with the key retrosynthetic fragment 115 being synthesised in 12 steps, yet with an excellent overall yield of $28 \%$, scheme 100 . The desired C-2 and C-3 syn relationship was established by a Sharpless asymmetric dihydroxylation. Again, a boron-mediated 1,5-anti aldol reaction was used to control the diastereoselectivity of the subsequent aldol reaction. As depicted in scheme 100, isopropylaldehyde was used as a model substrate, and although the desired anti adduct 123 was synthesised in $61 \%$ yield, due to the presence of the methoxy substituent at the $\beta$ position, the diastereoselectivities were again, rather modest.

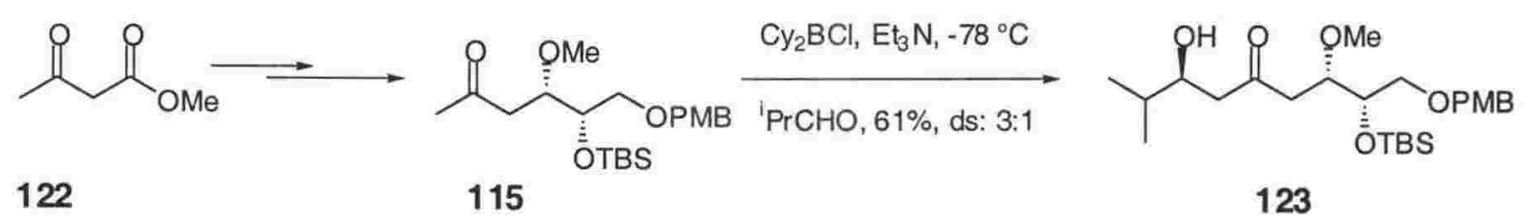

Scheme 100: Synthesis of the C-6 to C-1 fragment of peloruside A and model aldol studies

Shortly following Paterson's fragment synthesis of peloruside A, De Brabander, Liao and $\mathrm{Wu}$ published the first, and to date, only total synthesis of peloruside $\mathrm{A} .^{205}$ Although somewhat of a linear strategy was adopted, which in turn resulted in an overall yield of less than $2 \%$, this was nonetheless elegant work given the complexity of the target molecule. Additionally, by completing the first total synthesis, De Brabander was able to establish the absolute stereochemistry of peloruside $\mathrm{A}$ - this being the opposite to that initially reported by West and Northcote. ${ }^{15}$

Only two fundamental disconnects were made by De Brabander in his retrosynthetic analysis, figure 37. It is interesting to note that De Brabander's synthesis of the C-14 to C-24 side chain commenced with the zirconium catalysed ethylmagnesation of 2,5-dihydrofuran 124, in

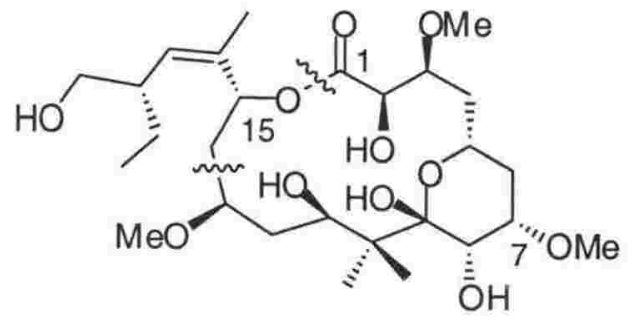

Figure 37: De Brabander's retrosynthesis 
much the same manner as that proposed by Stocker et. al. The homoallylic alcohol $\mathbf{1 2 5}$ was subsequently acylated with methacryloyl chloride, subjected to RCM (using Grubb's second generation catalyst), the resulting lactone treated with methyl lithium and the ensuing hydroxyl silylated to give $\mathbf{1 2 6}$ in an overall yield of approximately $17 \%$, scheme 101.

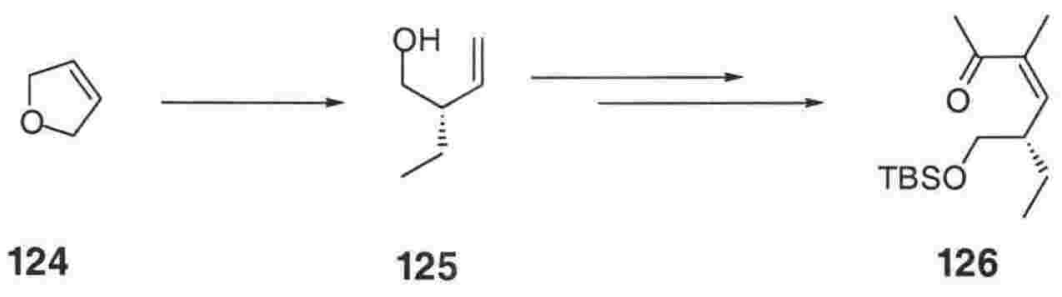

Scheme 101: De Brabander's synthesis of the C-14 to C-24 fragment of peloruside A

De Brabander's synthesis for the remainder of peloruside A began with the silylation and dihydroxylation of the previously reported alcohol 127 , scheme $102 . \mathrm{A} \mathrm{Pb}(\mathrm{OAc})_{4}$ assisted cleavage of the ensuing diol then gave alcohol 128. A five step, linear strategy was then followed to give dihydropyranone 129 , in $47 \%$ overall yield.

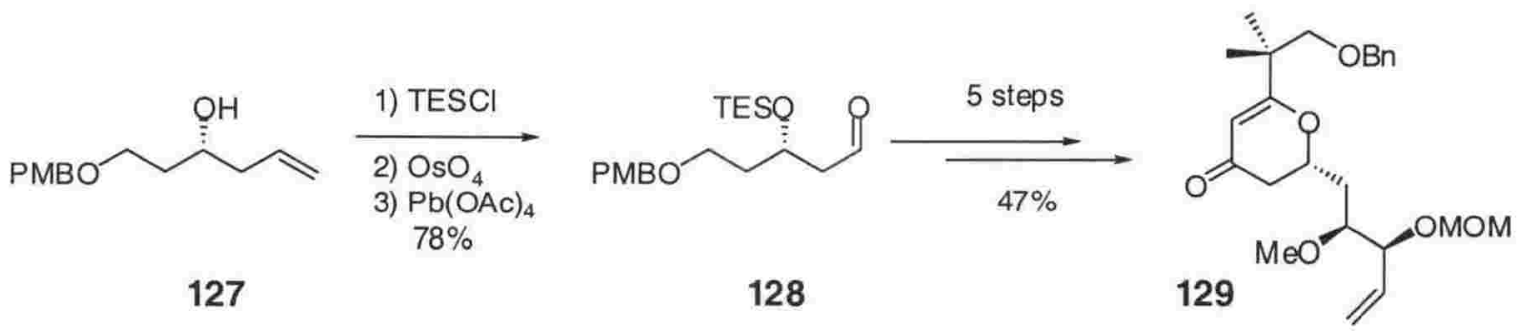

Scheme 102: De Brabander's synthesis of the key intermediate dihydropyranone 129.

Formation of 129 thus provided a key starting point for establishing the correct stereochemistry of the pyranose ring of peloruside A. A five step strategy involving a Luche reduction, a hydroxyl-directed epoxidation followed by an in situ methylation, selective methylation of the ensuing equatorial hydroxyl and finally silylation of the remaining axial hydroxyl gave pyranose 130, scheme 103. A series of functional group modifications then gave methyl ester $\mathbf{1 3 1}$. 


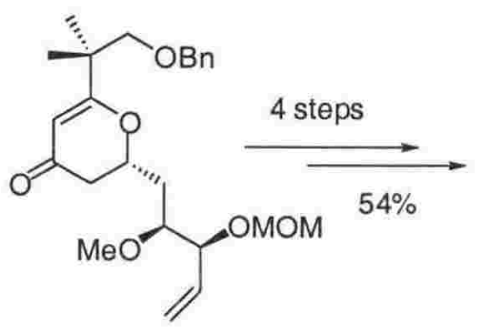

129

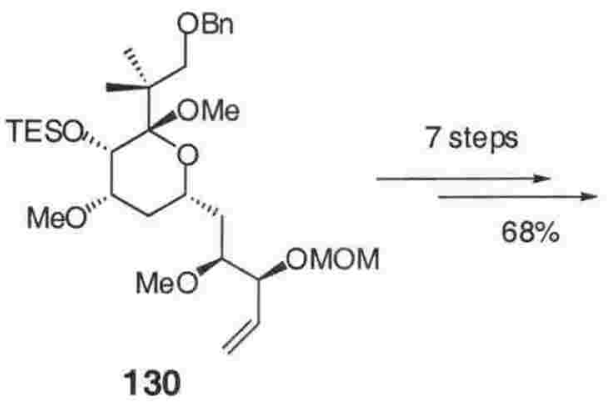

130

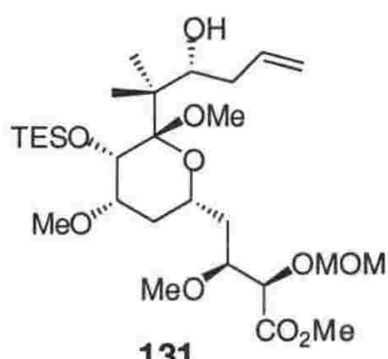

131

Scheme 103: De Brabander's synthesis of the key intermediate methyl ester $\mathbf{1 3 1 .}$

The final transformations required for De Branbander's synthesis of peloruside A first required a diastereoselective aldol reaction with the previously synthesised C-14 to C-24 fragment, scheme 104. Although the aldol reaction of $\mathbf{1 3 1}$ with $\mathbf{1 2 6}$ was rather unselective, the 2:1 ratio of diastereomers was nonetheless welcomed as previous problems had been encountered when attempting to synthesise peloruside by an alternative strategy. It is also interesting to note, that this aldol reaction was conducted in the presence of an unprotected hydroxyl group.
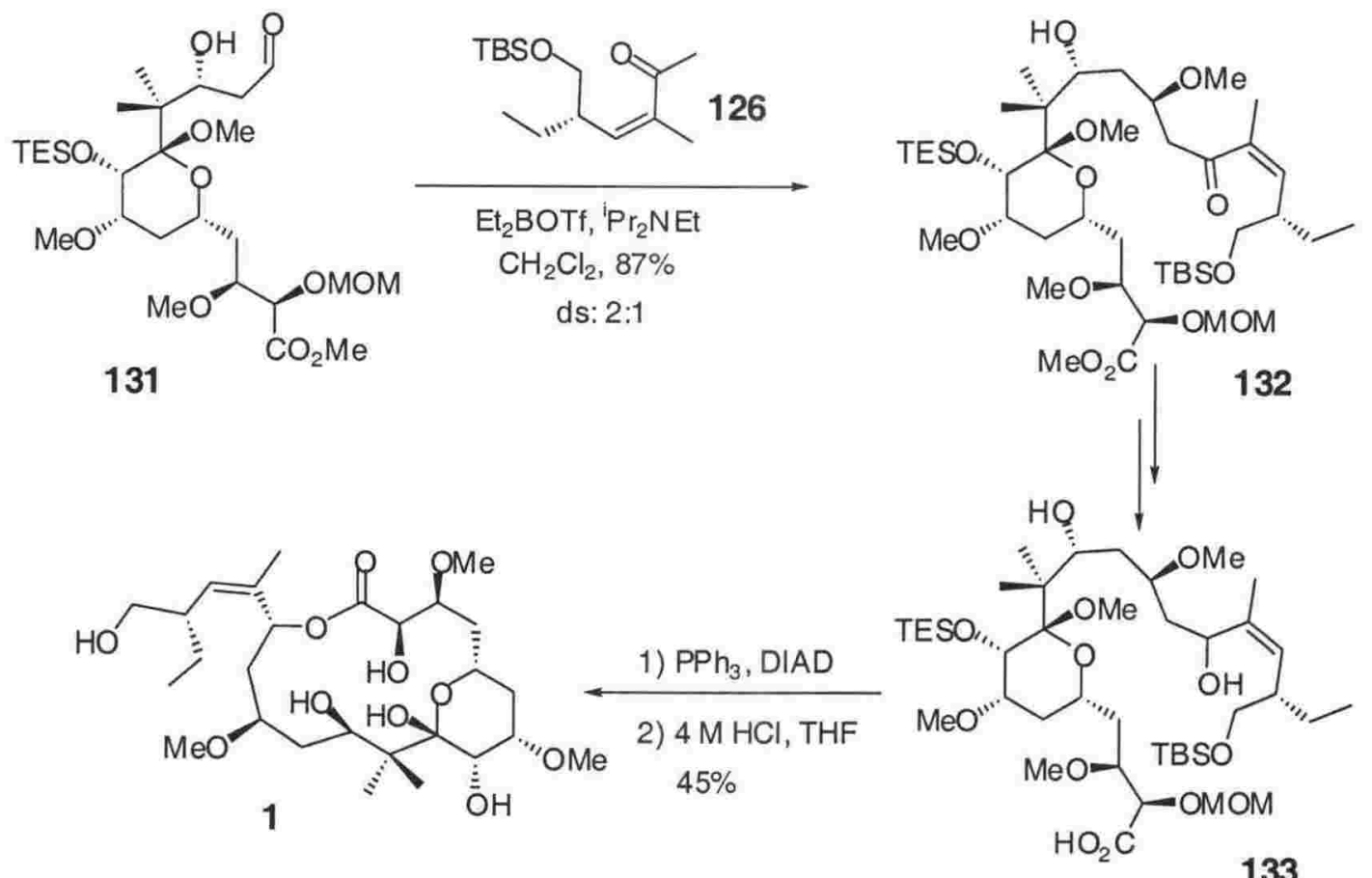

1) $\mathrm{PPh}_{3}$, DIAD
2) $4 \mathrm{M} \mathrm{HCl}, \mathrm{THF}$
$45 \%$

133

Scheme 104: The final chemical transformations required in De Brabander's total synthesis of peloruside $\mathrm{A}$ 
Perhaps the most salient feature of De Brabander's synthesis of peloruside A however, was the final Mitsunobu-type lactonisation, scheme 104. It was found that the same desired lactone was produced, regardless of the stereochemistry at C-15. It was subsequently speculated that geometrical/conformation constraints preclude formation of the $\mathrm{C}-15$ epimeric lactone, forcing the cyclisation to occur via an alkoxyphosphonium intermediate, or the less familiar, acyloxyphosphonium intermediate.

Following De Brabander's total synthesis of peloruside A, Ghosh and Kim reported, in two separate papers, a synthesis for the C-1 to C-9 and C-10 to C-24 fragments of peloruside A. ${ }^{207,}{ }^{208}$ These fragments are highlighted in scheme 105.

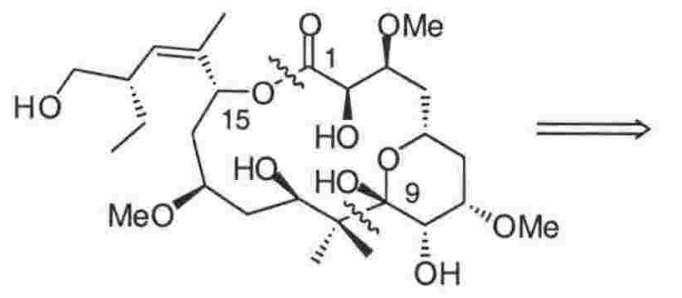$$
\text { H }
$$

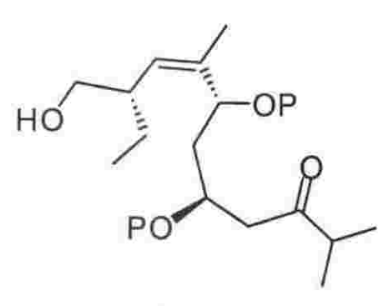

134

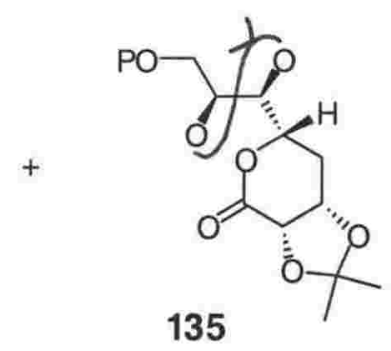

135

Scheme 105: Ghosh and Kim's retrosynthetic analysis of peloruside A

Of the two aforementioned fragments, synthesis of the C-1 to C-9 fragment $\mathbf{1 3 5}$ was reported first - commencing with the preparation of the $\alpha, \beta$-unsaturated ester 136 using known procedures. After installation of the C-2 to C-3 syn relationship using Sharpless asymmetric dihyroxylation methodology, a series of basic functional group transformations lead to aldehyde $\mathbf{1 3 7}$ in a $47 \%$ overall yield, scheme 106. Allylation of 137 using allyl magnesium bromide, followed by oxidation of the resulting alcohol gave ketone 138.

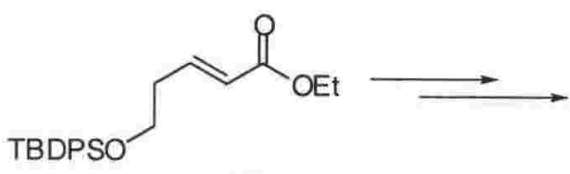

136

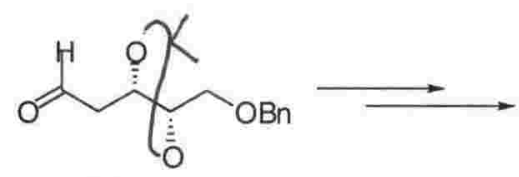

137<smiles>C=CCC(=O)C[C@@H](O)[C@@](C)(COCc1ccccc1)C(C)(C)C</smiles>

138

Scheme 106: Gosh and Kim's partial synthesis of the C-1 to C-9 fragment of peloruside 
A unique feature of this C-1 to C-9 fragment synthesis was the subsequent chelation controlled asymmetric reduction of ketone 138 using a solution of $\mathrm{LiAl}_{4}$ and $\mathrm{LiI}$ in diethylether, scheme 107. As depicted in transition state 138a, the observed diastereoselectivity can be rationalised by the presence of the gem-dimethyl group on the $\beta$-face, resulting in the reduction of the carbonyl from the less hindered $\alpha$-face. Subsequent esterification with acrolyl chloride and RCM of the corresponding acrylated ester provided the $\alpha, \beta$-unsaturated $\delta$-lactone 140. A six-step strategy was then used to convert 140 into the desired C-1 to C-9 fragment 135. Key features of this transformation include the saponification of lactone $\mathbf{1 4 0}$ and an asymmetric dihydroxylation (using A-D mix) to give the C-7 and C-8 syn relationship.

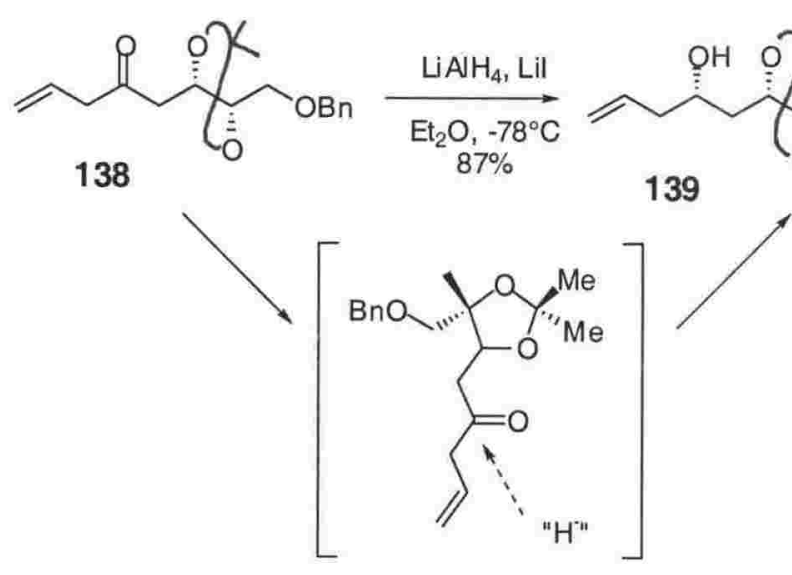

$138 \mathrm{a}$
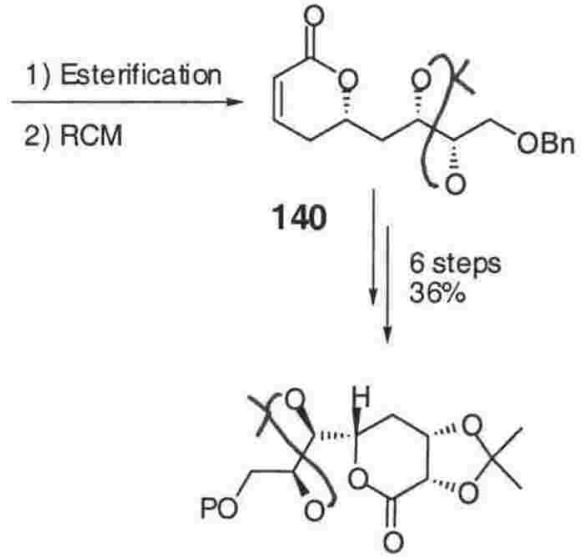

135

Scheme 107: Gosh and Kim's synthesis of the C-1 to C-9 fragment of peloruside A

Gosh's synthesis of the C-10 to C-24 fragment of peloruside A commenced with the asymmetric allylation of Evan's chiral oxazolidinone 144, using a slightly different procedure to that reported in this thesis for the synthesis of the enantiomerically pure side chain 39 (refer to page 37, chapter 2), scheme 108. Following some basic functional group transformations, the subsequent installation of the $\mathrm{Z}$ olefin using Horner-Emmons protocol and further functional group manipulations, the correct stereochemistry at C-15 was then established by using Brown's asymmetric allylboration protocol. Esterification and RCM protocol followed to give the $\alpha, B$-unsaturated lactone $\mathbf{1 4 2 .}$ 
<smiles>CC#CCCCC(=O)N1C(=O)OC[C@H]1Cc1ccccc1</smiles>

141<smiles>CC[C@H](/C=C(/C)[C@H]1CC=CC(=O)O1)COCc1ccccc1</smiles>

142
1) $\mathrm{H}_{2} \mathrm{O}_{2}, 6 \mathrm{~N} \mathrm{NaOH}$

2) $\mathrm{NaBH}_{4}, \mathrm{PeSeSePh}, \mathrm{AcOH}$

3)TBDPSCl
$74 \%$<smiles>CC[C@H](/C=C(/[C@H](CC)COc1ccccc1)[C@H](O)OCc1ccccc1)CC(=O)O</smiles>

143
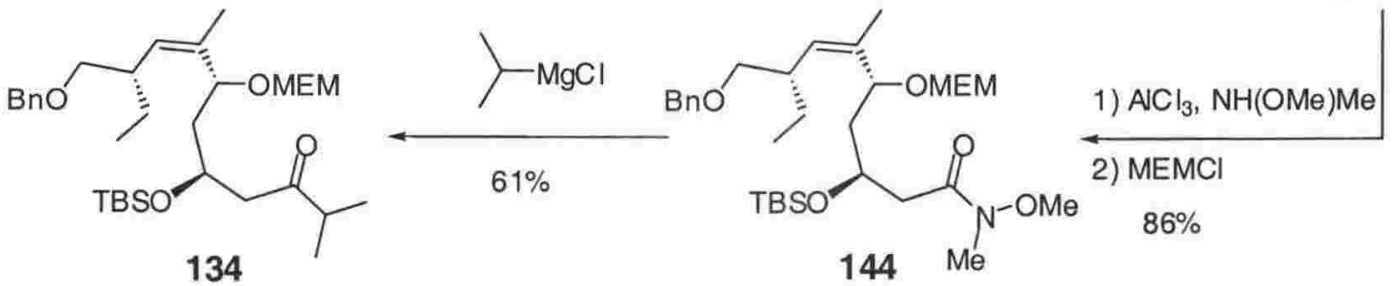

Scheme 108: Gosh and Kim's synthesis of the C-10 to C-24 fragment of peloruside A

An elegant feature of Gosh's strategy for the installation of the correct stereochemistry at C-13 involved the stereoselective epoxidation of $\mathbf{1 4 2}$ and treatment of the resulting epoxide with diphenyldiselenide and sodium borohydride, scheme 108. Protection of the ensuing alcohol then gave $\mathbf{1 4 3}$ in a $\mathbf{7 4 \%}$ overall yield for the three steps. It was initially anticipated that lactone 143 could be directly opened with isopropylmagnesium chloride, however this procedure proved elusive. Thus conversion of lactone $\mathbf{1 4 3}$ to the Weinred amide 144 was first required before synthesis of the final fragment 134 could be achieved.

Taylor and $\mathrm{Jin}^{209}$ reported the first partial synthesis of the correct enantiomer of peloruside A. Although De Brabander had determined the absolute stereochemistry of peloruside A, Taylor and Jin previously compated the structural and conformational features of peloruside A and epothilone B using NMR and computer modelling techniques. Their modelling results also indicated that the enantiomer opposite to that initially reported by West and Northcote ${ }^{15}$ was the correct enantiomer of peloruside A. Taylor and Jin initially focussed on the synthesis of the C-8 to C-24 fragment of peloruside A, as depicted in scheme 109. 

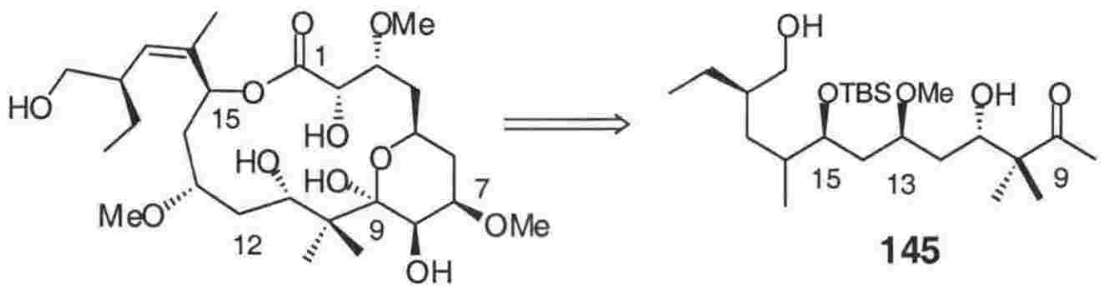

145

Scheme 109: Taylor and Jin's target C-8 to C-24 fragment of peloruside A

Taylor's synthesis of the C-8 to C-24 fragment of peloruside A commenced in a manner very similar to that previously reported by Gosh. Thus an asymmetric alkylation reaction using Evans's oxazolidinone, a Still-Gennari olefination and Brown's asymmetric allylation, were the key methodologies used during the nine-step synthesis of alcohol 147. Establishment of the 1,3-syn relationship between C-13 and C-15 was achieved by an elegant regioselective, iodine-induced, carbonate cyclization to give carbonate $\mathbf{1 4 8}$ in excellent yield, scheme 110. Exposure of $\mathbf{1 4 8}$ to basic methanol solution and protection efficiently produced the syn-epoxy ether 149. Ether 149 was then subjected to nucleophilic attack by the lithium anion of dithiane, methylated in situ, and the dithiane moiety hydrolysed to give aldehyde $\mathbf{1 5 0}$.<smiles>CCCC(=O)N1C(=O)OC[C@@H]1Br</smiles>

146

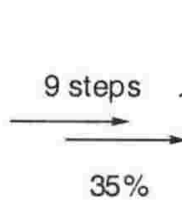<smiles>C=CC[C@H](O)/C(C)=C\C(CC)CO[Na]</smiles>

147

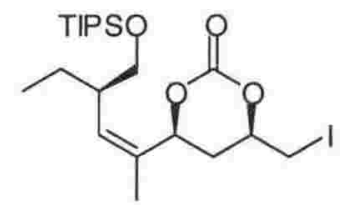

148

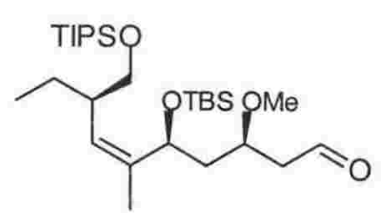

150
1) 1,3-dithiane, $n B$ uLi $\mathrm{HMPA},\left(\mathrm{CH}_{3} \mathrm{O}\right)_{2} \mathrm{SO}_{2}, 85 \%$

2) $\mathrm{Mel}, \mathrm{CH}_{3} \mathrm{CN} / \mathrm{H}_{2} \mathrm{O}, 90 \%$
1) Boc-ON, $91 \%$ 2) $\mathrm{NIS}, \mathrm{CH}_{3} \mathrm{CH}, 92 \%$<smiles>[B]OC(CC(CO)CO)/C(C)=C\C(CC)COC1CO1</smiles>

149

Scheme 110: Synthesis of key intermediate aldehyde 150. 
The final requirement in Taylor's synthesis of the C- 8 to C-24 fragment of peloruside A involved a Mukaiyama aldol reaction of the B-methoxy aldehyde $\mathbf{1 5 0}$ with the TMS silyl enol ether 151, scheme 111. It is interesting to note that even though an achiral lewis acid was used, good 1,3 anti diastereoselectivity was nevertheless observed in this reaction.

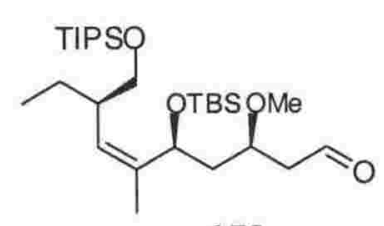

150

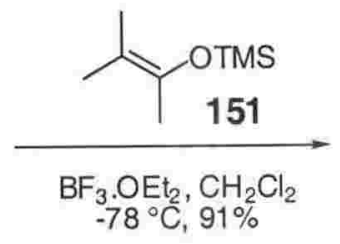

$-78^{\circ} \mathrm{C}, 91 \%$

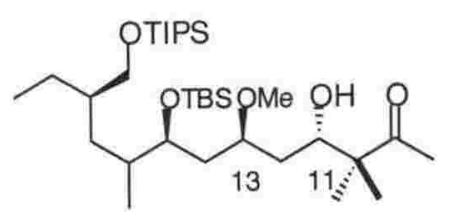

145 anti:syn (8:1)

Scheme 111: Synthesis of the C-8 to C-24 fragment of peloruside A

The final strategy towards the synthesis of peloruside A to be discussed in this thesis is the recently published partial synthesis of Pagenkopf, Engers and Bassindale. ${ }^{210}$ This strategy has similar disconnects to those proposed by Stocker, Teesdale-Spittle and Hoberg. ${ }^{193}$ These key disconnects are depicted in scheme 112.

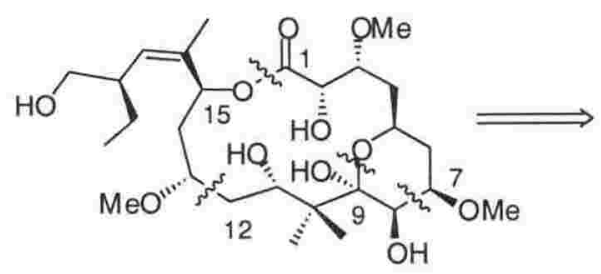

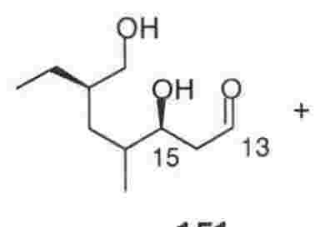

151

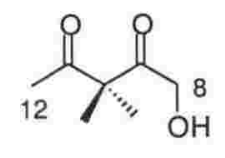

152

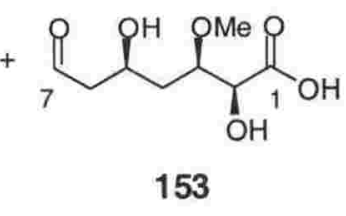

153

Scheme 112: Pagenkopf, Engers and Bassindale's retrosynthesis of peloruside A

The unique difference between Pagenkopf's strategy, and the other strategies reported for the synthesis of peloruside A however, is Pagenkopf's use of the commercially available triacetal D-glucal for providing the chiral backbone for the pyranose segment of peloruside A. This strategy results in an elegant approach towards the synthesis of peloruside A. 
Thus, triacetal D-glucal $\mathbf{1 5 4}$ was converted in five steps to the known pyranose $\mathbf{1 5 5}$, scheme 113. This transformation involved formation of a methoxy acetal, following treatment of glucal 154 with $10 \mathrm{~mol} \% \mathrm{Ph}_{3} \mathrm{P} \cdot \mathrm{HBr}$ and methanol, acetate cleavage, benzylidine acetal formation, methylation, and selective cleavage of the primary benzylidine acetal under Hanessian-Hullar radial bromination conditions. Allylation of 155, followed by dihydroxylation and $\mathrm{NaIO}_{4}$ oxidative cleavage of the resulting diol gave the desired C-1 to C-7 fragment 156. Indeed, this eight-step synthesis has been performed on the $50 \mathrm{~g}$ scale, with a respectable overall yield of $46 \%$.

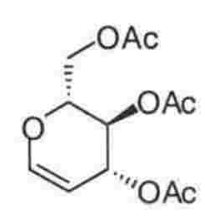

154

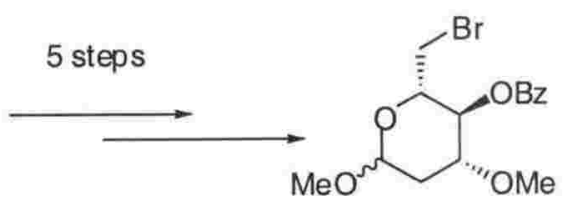

155
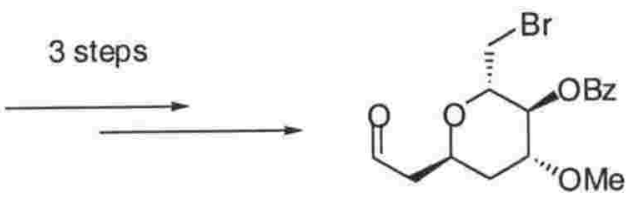

156

Scheme 113: Pagenkopf's synthesis of the C-1 to C-7 fragment of peloruside A

An equivalent of the central C-8 to C-12 piece was synthesised in $69 \%$ overall yield by the $S_{\mathrm{E}} 2^{\prime}$ addition of an allylic stannane generated in situ from Barbier-type reaction of 157 with $\mathrm{SnCl}_{2} .2 \mathrm{H}_{2} \mathrm{O}$ and benzyloxy acetaldehyde 158 , scheme 114 . A Swern oxidation then completed the synthesis of 159 .

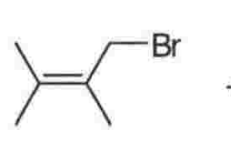

157<smiles>O=CCOCc1ccccc1</smiles>

158

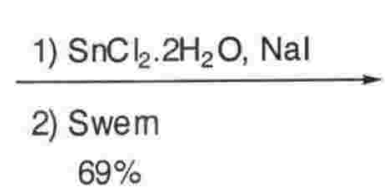

$69 \%$

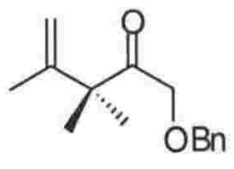

159

Scheme 114: Synthesis of the C-8 to C-12 fragment

Perhaps the most challenging step in Pakenkopf's strategy was in achieving the desired 1,2-anti relationship between C-7 and C-8 during the coupling of 156 and 159. Thus a range of Lewis acids were explored during the coupling of $\mathbf{1 5 6}$ and the TMS enolate 159a, with $\mathrm{BF}_{3}$. OEt giving the best, but still modest, diastereoselectivity, scheme 115 . 
<smiles>C=C(C)C(C)(C)/C(=C\OCc1ccccc1)O[Na]</smiles>

$159 a$

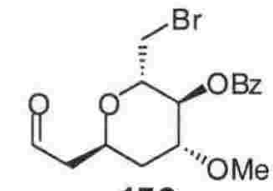

156

$\mathrm{BF}_{3}$. OEt, $\mathrm{CH}_{2} \mathrm{Cl}_{2}$

Scheme 115: Establishment of the C-7 to C-8 anti relationship

Diastereomer 160a was then methylated and subjected to a suspension of zinc and copper in ethanol which resulted in Vasella ring cleavage by spontaneous B-elimination of the alkyl zinc generated in situ by reduction of the alkyl bromide with the zinc-copper couple, scheme 116. For ease of characterisation, it was then anticipated that conditions could be established such that equilibration would favour the hemiacetal $\mathbf{1 6 2}$. Unfortunately though, reliably obtaining $\mathbf{1 6 2}$ proved elusive, indicating that the pyranose fragment of peloruside may first need to be deprotected for cyclisation to occur.

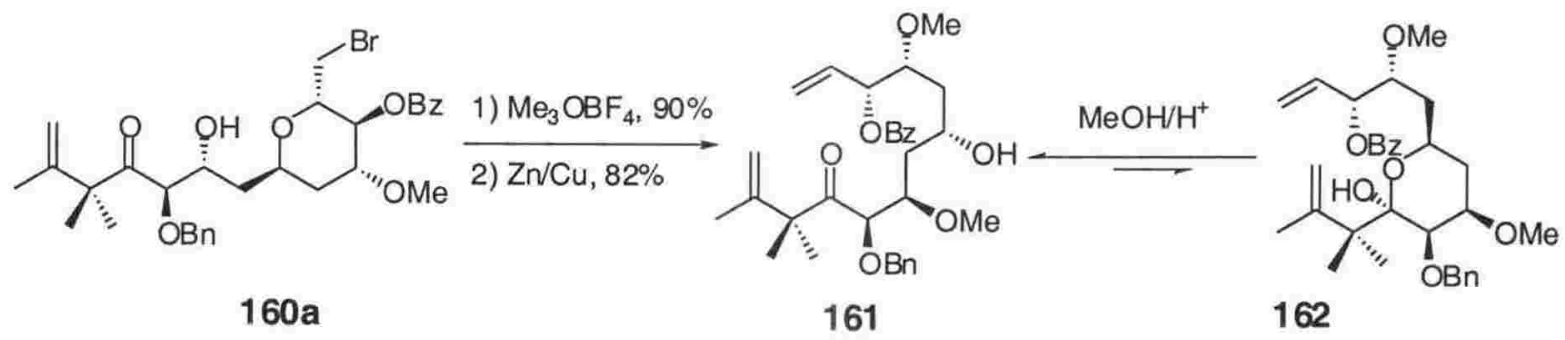

Scheme 116: Attempted cyclisation of the C-1 to C-12 fragment of peloruside A

Finally, a series of basic functional group manipulations involving an ozonolysis, oxidation, and esterification of the resulting carboxylic acid gave the complete $\mathrm{C}-1$ to $\mathrm{C}$ 12 carbon framework, scheme 117. By comparison to the previous synthesises of peloruside A, establishment of the correct stereochemistry in the C-5 to C-10 region appears to be the most challenging aspect of the synthesis. Thus the strategy adopted by Pakenkopf, Engers and Bassindale represents an elegant solution to this problem. 


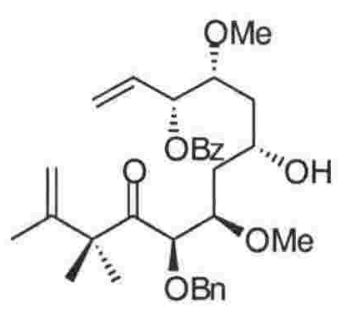

161

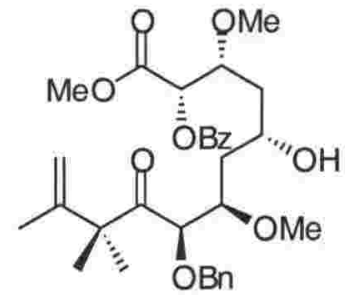

163

Scheme 117: Pagenkopf's synthesis of the C-1 to C-12 fragment of peloruside A 


\section{References}

1. Nicolaou, K.C.; Roschangar, F.; Vourloumis, D. Angew. Chem., Int. Ed. Engl. 1998, 37, 2014.

2. Information retrieved from the Internet homepage of the Cancer Society of New Zealand.(Acurate as of 01/01/04) http:www.cancernz.org.nz

3. Wani, M. C.; Taylor, H. L.; Wall, M. E.; Coggon, P.; McPhail, A. T. J. Am. Chem. Soc. 1971, 93, 2325.

4. Pettit, G. R.; Herald, C. L.; Doubek, D. L.; Herald, D. L. J. Am. Chem. Soc. 1982, 104, 6846.

5. Schiff, P. B.; Fant, J.; Horwitz, S. B. Nature 1979, 277, 665.

6. Reinecke, P.; Schmitz, M.; Schneider, E. M.; Gabbert, H. E.; Gerharz, C. D. Cancer Invest. 2000, 18, 614.

7. Fahey, J. Taxol's Next Stand, Forbes; New York; 2001.

8. Cowden, C.J; Paterson, I. Nature (Lond.) 1997, 387, 238.

9. Giannakakou, P.; Sackett, D.L.; Kang, Y.K.; Zhan, Z.; Buters, J. T. M.; Fojo, T.; Poruchynsky, M.S. J. Biol. Chem. 1997, 272, 17118.

10. Rowinsky, E.K.; Einsenhauer, E.A.; Chaudhry, V.; Arbuck, S.G.; Donehower, R.C. Semin. Oncol. 1993, $20,1$.

11. Rowinsky, E.K.; Donehower, R.C. J. Natl. Cancer Inst. 1991, 83, 1778.

12. Höfle, G.; Bedorf, N.; Gerth, K.; Reichenbach H. (GBF), DE-B 4138042, 1993, [Chem. Abstr. 1993, 120, 52841.]

13. Bollag, D.M; McQueney,P.A.; Zhu, J.; Hensens, O.; Koupal, L.; Liesch, J.; Goetz, M.; Larzarides, E.; Woods, C.M. Cancer Res. 1995, 55, 2325.

14. Kowalski, R.J.; Giannakakou, P.; Hamel, E. J. Biol. Chem. 1997, 272, 2534.

15. West, L. M.; Northcote, P. T. J. Org. Chem. 2000, 65, 445.

16. Hood, K. A.; Bäckström, B. T.; West, L. M.; Northcote, P. T.; Berridge, M. V.; Miller, J. H. Anti-Cancer Drug Design, 2001, 16, 155.

17. Hood, K.A.; West, L. M.; Rouwé, B.; Northcote, P. T.; Berridge, M. V.; Wakefield, St. J.; Miller, J. H. Cancer Res. 2002, 62, 3356. 
18. Mooberry, S.L.; Tein, G.; Hernandez, A. H.; Plubrukarn, A.; Davidson, B. S. Cancer Res. 1999, 59, 653.

19. Long, B. H.; Carboni, J. M.; Wasserman, A. J.; Cornell, L. A.; Casazza, A. M.; Jensen, P. R., Lindel; T., Fenical, W.; Fairchild, C. R. Cancer Res. 1998, 58, 1111.

20. Combeau, C; Provost, J.; Lancelin, F.; Tournoux, Y.; Prod'Homme, F.; Herman, F.; Lavelle, F.; Leboul, J.; Vuilhorgne, M. Molecular Pharmacology, 2000, 57, 553.

21. Ter Haar, E.; Kowalski, R. J.; Hamel, E.; Lin, C. M.; Longley, R. E.; Gunasekera, S. P.; Rosenkranz, H. S.; Day, B. W. Biochemistry 1996, 35, 24.

22. Kowalski, R.J.; Giannakakou, P.; Gunasekera, S.P.; Longley, R.E.; Day, B. W.; Hamel, E. Molecular Pharmacology 1997, 52, 613.

23. Kawamura, K. I.; Grabowski, D.; Weizer, K.; Bukowski, R.; Ganapathi, R, J. Br. J. Cancer 1996, 73, 183.

24. Martello, L.A.; McDaid, H. M.; Regl, D.L.; Yang, C.P.; Meng, D.; Pettus, T.R.; Kaufman, M. D.; Arimoto, H.; Danishefsky, S. J.; Smith III, A. B.; Horwitz, S. B. Clin. Cancer Res. 2000, 6, 1978.

25. Northcote, P. T.; personal communication.

26. Nogales, E.; Wolf, S.; Downig, K.H. Nature 1998, 391, 199.

27. Margollis, R.L.; Wilson, L. Cell 1978, 13, 1.

28. Nogales, E.; Wolf, S.; Downig, K.H. Nature 1998, 391, 199.

29. Mitchison, T.; Kirschner, M.W.; Nature 1984, 312, 237.

30. Nogales, E. Annu. Rev. Biochem. 2000, 69, 277.

31. a) Kirschner, M.W., Mitchinson, T. Cell 1986, 45, 329. b) Kirschner, M.W., Mitchinson, T. Nature 1986, 324, 621.

32. McIntosh, J. M.; Hering, G. E. Annu. Rev. Cell Biol. 1991, 7, 403.

33. Brinkley, B.R. Annu. Rev. Cell Bio. 1985, 1, 145.

34. a) McIntosh, R.J.; Grishchuk, E. L.; West, R.R. Annu. Rev. Cell Dev. Biol. 2002, 18,193 b) Russell, P.J. Genetics, 1998, 5th Ed, Benjamin Cummings, 50-56.

35. Lobert, S.; Ingram, J. W. Correia J. J.; Cancer Research 1999, 59, 4816.

36. Wilson, L.; Jordan, M.A. Chem. Biol. 1995, 2, 569.

37. Sackett, D. L. Pharmacol. Ther. 1993, 59, 163.

38. Hamel, E. Pharmacol. Ther. 1992, 55, 31. 
39. Yamada, K.; Kigoshi, H. Bull. Chem. Soc. Jpn. 1997, 70, 1479.

40. Pettit, G. R. Prog. Chem. Org. Nat. Prod. 1997, 70, 1.

41. Smith, C. D.; Zhang, X.; Mooberry, S. L.; Patterson, G. M. L.; Moore, R. E. Cancer Res. 1994, 54, 3779.

42. Fighetti, M.; Rubino, S.; Cappuccinelli, P.; Monaco, G. Microbiologica (Bologna), 1988, 11, 269.

43. Gerwick, W. H.; Proteau, P. J.; Nagle, D. G.; Hamel, E.; Blokin, A.; Slate, D. L. J. Org. Chem. 1994, 59, 1243.

44 Schiff, P.B.; Horwitz, S. B. Proc. Natl. Acad. Sci. USA, 1980, 77, 1561.

45. Dustin, P. Microtubules $2^{\text {nd }}$ Edition, Springer-Verlag, Berlin; 1984, 484.

46. Derry, W.B.; Wilson, L.; Jordan, M.A. Biochem. 1995, 34, 2203.

47. Bollag, D. M.; McQueney, P. A.; Zhu, J.; Hensens, O.; Koupal, L.; Liesch, J.; Goetz, M.; Lazarides, E.; Woods, C. M. Cancer Res. 1995, 55, 2325.

48. Nicolaou, K.C.; Ritzén, A.; Namoto, K. Chem. Commun. 2001, 1523.

49. ter Haar, E.; Rosenkranz H. S.; Hamel, E., Day, B.W. Bioorg. Med. Chem. 1996, $4,1659$.

50. Lindel, T.; Jensen, P.R.; Fenical, W.; Long, B. H.; Casazza, A. M.; Carboni, J.; Fairchild, C.R. J. Am. Chem. Soc. 1997, 119, 8744.

51. a) Dhamodharan, R.; Jordan, M. A.; Thrower, D.; Wilson, L.; Wadsworth, P. Mol. Biol. Cell 1995, 6, 1215. b) Jordan, M. A.; Toso, R. J.; Thrower, D.; Wilson, L. Proc. Nat. Acad. Sci (Wash.), 1993, 90, 9552. c) Jordan, M. A.; Wilson, L. Curr. Opin. Cell Biol. 1998, 10, 123. d) Jordan, M.A.; Thrower, D.; Wilson, L. Cancer Res. 1991, 51, 2212. e) Jordan, M. A.; Thrower, D.; Wilson, L. J. Cell Sci. 1992, 102,401. f) Toso, R. J.; Jordon, M. A.; Farrell, K. W.; Matsumoto, B.; Wilson, L. Biochemistry, 1993, 32, 1285.

52. Caplow, M.; Zeeberg, B. Eur. J. Biochem. 1982, 127, 319.

53. Wilson, L., Miller, H.P., Farrell, K. W., Synder, K. B., Thompson, W. C., Purich, D. L., Biochemistry, 1985, 24, 5254.

54. Abal, M.; Andreu, J.M.; Barrasoain, I. Curr. Cancer Drug Targets 2003; 3, 193.

55. Trielli, M.O.; Andreassen, P.R.; Lacroix, F.B.; Margolis, R.L. The Journal of Cell Biology 1996, 135, 689.

56. Hartwell, L.H.; Weinert, T.A. Science (Wash. DC). 1989, 246, 629.

57. Blagosklonny, M. V.; Fojo, T. Int. J. Cancer, 1999, 83, 151. 
58. Blagosklonny, M. V.; Giannakakou, P.; El-Deiry, W. S.; Kingston, D. G. I.; Higgs, P. I.; Neckers, L.; Fojo, T. Cancer Res. 1997, 57, 130.

59. Haldar, S.; Basu, A.; Croce, C. M. Cancer Res. 1997, 57, 229.

60. Bhalla, K.; Ibrado, A. M.; Tourkina, E.; Tang, C.; Maloney, M. E.; Huang, T. Leukemia 1993, 7, 1028.

61. Woods, C.M.; Zhu, J.; McQueeny, P. A.; Bollag, D.; Lazarides, E. Mol. Med., 1995, 1, 506.

62. Ginnakakou, P.; Robery, R.; Fojo, T.; Blagosklonny; M.V. Oncogene 2001, 20, 3806.

63. Hood, K.A. PhD Thesis, Victoria University of Wellington, 2002.

64. Herst, P, Tan, A, Berridge, M. Unpublished observation.

65. West, L.M. PhD Thesis, Victoria University of Wellington, 2001.

66. Wilson, L.; Meza, I. J. Cell Biol. 1973, 58, 709.

67. a) Nogales, E.; Wolf, S. G.; Khan, I. A.; Luduena, R. F.; Downing, K. H. Nature (Lond.), 1995, 375, 424. b) Nogales, E.; Wolf, S. G.; Downing, K. H. J. Struct. Biol. 1997, 118, 119.

68. Parness, J.; Horwitz, S. B. J. Cell Biol. 1981, 91, 479.

69. Kowalski, R.J.; ter Haar, E.; Longley, R.E.; Gunasekera, S. P.; Lin, C.M.; Day, B.W., Mol. Biol. Cell, 1995, 6, 368. b) Kowalski, R.J.; Giannakakou, P.; Gunasekera, S.P.; Longley, R.E.; Day, B. W.; Hamel, E. Molecular Pharmacology, 1997, 52, 613.

70. Pryor, D.E.; O’Brate, A.; Bilcer, G., Diaz, J., F.; Wang, Y.; Wang, Y.; Kabaki, M.; Jung, M. K.; Andreu, J. M.; Ghosh, A. K.; Giannakakou, P.; Hamel, E. Biochemistry, 2002, 41, 9109.

71. Horwitz S. B. Trends Pharmacol Sci. 1992, 13, 134.

72. Giannakakou, P.; Sackett, D.L.; Kang, Y.K.; Zhan, Z.; Buters, J. T. M.; Fojo, T.; Poruchynsky, M.S. J. Biol. Chem. 1997, 272, 17118.

73. a) Gottesman, M. M.; Pastan, I. Annu. Rev. Biochem. 1993, 62, 385. b) Bradley, G.; Ling, V. Cancer Metastasis Rev. 1994, 13, 223.

74. a) Giannakakou, P.; Gussio, R.; Nogales, E.; Downing, K. H.; Zaharevitz, D.; Bollbuck, B.; Poy, G.; Sackett, D.; Nicolaou, K. C.; Fojo, T. Proc. Natl. Acad. Sci. U.S.A., 2000, 97, 2904. b) Kowalski, R. J.; Giannakakou, P.; Hamel, E. J. Biol. Chem. 1997, 272, 2534. c) Nicolaou, K.C.; Winssinger, N.; Pastor, J.;

Ninkovic, S.; Sarabia, F.; He, Y.; Vourloumis, D.; Yang, Z.; Li, T.; Giannakakou, P.; Hamel, E. Nature (Lond.), 1997, 387, 268. 
75. Jordan, A.; Hadfield, J. A.; Lawrence, N. J.; McGown, A. T. Med. Res. Rev. 1998, $18,259$.

76. Baguley, B. Auckland Cancer Research Centre, unpublished observations.

77. Ohtani; I.; Kusumi, T.; Kashman, Y.; Kakisawa, H. J. Am. Chem. Soc., 1991, 113, 4092.

78. a) Dale, J.A.; Mosher, H.S. J. Am. Chem. Soc. 1973, 95, 512. b) Sullivan, G.R.; Dale, J.A.; Moscher, H.S. J. Org. Chem. 1973, 38, 2143.

79. Ihara, M.; Setsu, F.; Shohda, M.; Taniguchi, N.; Tokunaga, Y.; Fukumoto, K.; J. Org. Chem. 1994, 59, 5317.

80. Smith, A.B.; Safonov, I.G.; Corbett, R.M.; J. Am. Chem. Soc. 2001, 123, 12426.

81 Procter, G. Asymmetric Synthesis, Oxford University Press, 1996, pg 69-73, 204-5

82. a) Reetz, M.T. Angew. Chem. Int. Ed. Engl. 1984, 23, 556. b) Masamune, S.;

Ellingboe, J.W.; Choy, W. J. Am. Chem. Soc. 1982, 104, 5526. c) Masumune, S.; Choy, W. Aldrichimica Acta, 1982,15, 47.

83. a) Zimmerman, H.E.; Traxler, D.M. J. Am. Chem. Soc. 1957, 79, 1920; b) Procter, G. Asymmetric Synthesis, Oxford University Press, 1996, pg 29, 72

84. a) Wood, A.I.; Holt, D.J.; Dominguez, M.C.; Jenkins, P.R. J. Org. Chem. 1998, 63, 8522. b) Hanessian, S.; Pougny, R.; Boessenkool, I.K. Tetrahedron 1984, 40, 1289.

85. Robinson, A. G.; Blood, A. E.; Hagemeyer Jr., H. J. (Eastman Kodak Company, Rochester, N.Y.), Dihydro-5-hydroxy-4,4-dialkyl-3-(2H)-furanones, US patent: \# 3,558,664, filed Feb., 12, 1969, Ser.no. 798,810.

86. a) Corey, E.J.; Venkateswarlu, A. J. Am. Chem. Soc., 1972, 94, 6190. b) Chaudhary, S.K.; Hernandez. O. Tetrahedron Lett., 1979, 99. c) Ogilvie, K.K.; Shifman, A.L.; Penney, C.L. Can. J. Chem., 1979, 57,2230.

87. a)Takai, K.; Heathcock, C. H. J. Org. Chem. 1985, 50, 3247 b) Shimizu, M.; Ishida, T.; Fujisawa, T. Chem. Lett. 1994, 1403 c) Waldemar, A.; Arias Encarnacion, L.A. Synthesis 1979, 388 d) Davies, S. G.; Middlemiss, D.; Naylor, A.; Willis, M. Terahedron. Lett. 1989, 30, 2971

88. (a) Brown, H.C; Jadhav, P.K. J. Am. Chem. Soc.1983, 105, 2092; (b) Brown, H.C.; Jadhav, P.K.; Perumal, P.T. Tetrahedron Lett. 1984, 25, 5111; (c) Brown, H.C.; Jadkav, P.K.; Bhat, K.S. J. Am. Chem. Soc. 1988, 110, 1535.

89. Harrison, B. A.; Verdine, G. Org. Lett., 2001, 3, 2157.

90. Boiteau, J., Van de Weghe, P., Eustache, J. Tetrahedron Lett., 2001, 42, 239. 
91. Hoye, T.R.; Caruso, A.J.; Dellaria Jr, J. F.; Kurth, M. J. J. Am. Chem. Soc. 1982, 104,6704 .

92. Mancuso, A.J.; Huang, S.-L.; Swern, D. J. Org. Chem. 1978, 43, 2480.

93. a)Mikami, K.; Matsukawa, S. J. Am. Chem. Soc. 1994, 116, 4077. b) Mikami, K.; Matsukawa,S.; Nagashima, M.; Funabashi, H.; Morishima, H. Tetrahedron Lett., 1997, 38, 579. c) Keck, G.E.; Krishnamurthy, D. J. Am. Chem. Soc. 1995, 117, 2363.

94. a) Torisawa, Y.; Shibasaki, M.; Ikegami, S. Chem. Pharm. Bull. 1983, 31, 2607.

b) Wood, W.W.; Rashid, A.; Tetrahedron Lett. 1987, 28, 1933. c) Hatakeyama, S.; Irie, H.; Shintani, T.; Noguchi, Y.; Yamada, H.; Nishitawa, M. Tetrahedron 1994, 50, 13369. d) Shekani, M.S.; Khan, K.M.; Mahmood, K.; Shah, P.M.; Malik, S. Tetrahedron Lett. 1990, 31, 1669.

95. a) Evans, D.A.; Bartroli, J.; Shih, T.L. J. Am. Chem. Soc. 1981, 103, 2127. b) Evans, D.A.; Rieger, D.L.; Bilodeau, M.T.; Urpi, F. J. Am. Chem. Soc. 1991, 113,1047. c) Crimmins, M.T; King, B.W; Tabet, E.A.; Chaudhary K.; J. Org. Chem 2001, 66, 894. d) Keck, G.E.; Palani, A.; McHardy, S.F. J. Org. Chem. 1994, 59, 3113.

96. Inanaga, J.; Hirata, K.; Saeki, H.; Katsuki, T.; Yamaguchi, M. Bull. Bull. Chem. Soc. Jap. 1979, 52, 1989.

97. Evans, D.A.; Hoyveda, H.A. J. Am. Chem. Soc. 1990, 112, 6447.

98. McDougal, P.G.; Rico, J.G.;Young, I.O.; Condon, B.D. J. Org.Chem. 1986, 51, 3388.

99. (a) Dess, D. B.; Martin, J. C. J. Am. Chem. Soc., 1991, 113, 7277. (b) Ireland, R. E.; Longbin L. J. Org. Chem., 1993, 58, 2899.

100. Gaucher, A.; Ollivier, J.; Marguerite, J.; Paugam, R.; Salaun, J. Can. J. Chem. 1994, 72, 1312.

101. Greene, T. W.; Wuts, P. G. M. Protective Groups in Organic Synthesis, John Wiley \& Sons, 1999, pg 127.

102. Nakano, T.; Terada, T.; Yasutake, I.; Ogawa, M. Synthesis, 1986, 9, 774.

103. For examples see; (a) Kosley, R.W., Jr.; Cherill, R.J. J. Org. Chem., 1989, 54, 2972; (b) Bhakuni, D.S.; Jain, S. Tetrahedron, 1980, 36, 3107; (c) Chatterjee, A.; Majumder, P.L.; Mukhergee, R.; Saha, S.K.; Talapatra, S.K. Tetrahedron Lett., $1965,21,1539$.

104. Stahi, E. Thin Layer Chromatography, $2^{\text {nd }}$ ed, 1967, Springer-Verlag.

105. (a)Georg, G.I.; Kant, J.; Gill, H.S. J. Am. Chem. Soc.; 1987, 109, 1129; (b) Mori, Y.; Kohchi, Y.; Suzuki, M. J. Org. Chem.; 1991, 56, 631;(c)Demuth, M.; Ritterskamp, P.; Weigt, E.; Schaffner, K. J. Am. Chem.; 1986, 108, 4149. 
106. Wittig, P. Pure Appl. Chem. 1964, 9, 245.

107. Vassilikogiannakis, G.; Chronakis, N. J. Am. Chem. Soc. 1998, 120, 9911.

108. Lavallée, P.; Ruel, R.; Grenier, L.; Bissonnette, M. Tetrahdron Lett, 1986, 27, 679.

109. Wilson, N. S.; Keay, B. A. J. Org. Chem., 1996, 61, 2918.

110. Batchelor, R.; Victoria University of Wellington; unpublished observations.

111. (a) Wuts, P. G. M.; Bigelow, S. S.; J. Org. Chem., 1988, 53, 5023; (b) Franke, F.; Guthrie, R. D. Aust. J. Chem., 1978, 31, 1285; (c) Torisawa, Y.; Shibasaki, K.; Ikegami, S. Tetrahedron Lett., 1979, 1865; (d) Ogilvie, K. K.; Beaucage, S. L.; Schifman, A. L.; Theriaul, N. Y.; Sadana, K. L. Can. J. Chem., 1978, 56, 2768; (e) Jones, S. S.; Reese, C. B. J. Chem. Soc., Perkin Trans. 1, 1979, 2762.

112. (a) Crich, D.; Ritchie, T. J. Carbohydr. Res., 1990, 197, 324, and references cited therein; (b) Friesen, R. W.; Daljeet, A. K. Tetrahedron Lett., 1990, 31, 6133.

113. Lavallée, P.; Ruel, R.; Grenier, L.; Bissonnette, M. Tetrahedron. Lett., 1986, 27, 679.

114. Chandrasekhar, S.; Mohanty, P. K.; Ramachander, T. Synlett. 1999, 7, 1063

115. March, J. Advanced Organic Chemistry: Reactions, Mechanisms, and Structure, McGraw-Hill, 1968, p 317.

116. Gigg, R.; Warren, C. D. J. Chem. Soc., 1968, 1903.

117. Cunningham, J.; Gigg, R.;Warren, C. D. Tetrahedron Lett., 1964, 1191.

118. Boss, R.; Scheffold, R. Angew. Chem., Int. Ed. Engl. 1976, 15, 558.

119. Nakayama, K.; Uoto, K.; Higashi, K.; Soga, T.; Kusama, T. Chem. Pharm. Bull., 1992, 40, 1718.

120. Corey, E.J.; Suggs, W.J. J. Org. Chem., 1973, 38, 3224.

121. Thomas, R. M.; Mohan, G. H.; Iyengar, D. S. Tetrahedron Lett., 1997, 38, 4721.

122. Kariyone, K.; Yazawa, H. Tetrahedron Lett., 1970, 2885.

123. Kadman, S. M.; Nayak, S. K.; Banerji, A. Tetrahedron Lett., 1992, 33, 5129.

124. Smith, A. B.; Rivero, R. A.; Hale, K. J.; Vaccaro, H. A. J. Am. Chem. Soc., 1991, 113,2092

125. Mereyala, H. B.; Guntha, S. Tetrahedron Lett., 1993, 34, 6929.

126. Gulab, S. A.; personal communication 
127. Choudary, B. M.; Prasad, A. D.; Swapna, V.; Valli, V. L. K.; Bhuma, V. Tetrahedron, 1992, 48, 953.

128. Choudary, B. M.; Prasad, A. D.; Bhuma, V.; Swapna, V. J.Org.Chem., 1992, 57, 5841.

129. Greene, T. W., Wuts, P. G. M., Protective groups in organic synthesis, John Wiley \& Sons, 3rd Edition, 1999, pgs 68-72.

130. Miyashita, M.; Hara. S.; Yoshikoshi, A. J. Org. Chem., 1987, 52, 2602.

131. a) Richardson, D. P.; Wilson, W.; Mattson, R. J.; Powers, D. M.; Dolan, B. T. J. Chem. Ed., 1991, 68, 951. b) Mitsunobu, O. Synthesis, 1981, 1.

132. Poos, G.I; Arth, G.E.; Beyler, R.E.; Sarett, L.H.; J. Am. Chem. Soc. 1953,75, 422

133. (a) Czernecki, S.; Georgoulis, C.; Stevens, C. L.; Vijayakumaran, K. Tetrahedron Lett. 1985, 26, 1699; (b)Herscovici, J.; Antonakis, K. J. Chem. Soc., Chem. Commun. 1980, 561; (c) Hersocovici, J.; Egron, M-J.; Antonakid, K. J. Chem. Soc. Perkin Trans. I. 1982, 1967. (d) Defaye, J.; Gadelle, A.; Angyal, S. J. Carbohydr. Res. 1984, 126, 165.

134. Honda, M.; Hiromasa, M.; Nagakura, I.; J. Org. Chem. 1997, 62, 8932.

135. Opatz, T.; Kunz, H. Tetrahedron Lett. 2000, 41, 10185.

136. Baars, S.M.; Hoberg, J.O. Personal communication.

137. Smith, A. B.; Levenberg, P. A. Synthesis, 1981, 567.

138. (a) Schrock, R.R.; Murdzek, J. S.; Bazan, G. C.; Robbins, J.; Dimare, M., O'Regan, M. J. Am. Chem. Soc. 1990, 112, 3875. (b) Bazan, G. C.; Khosravi, E.; Schrock, R. R.; Feast, W. J.; Gibson, V. C.; O’Regan, M. B.; Thomas, J. K.; Davis, W. M. J. Am. Chem. Soc. 1990, 112, 8378. (c) Bazan, G. C., Oskam, J. H.; Cho, H. N.; Park, L. Y.; Schrock, R. R. J. Am. Chem. Soc. 1991, 113, 6899.

139. (a) Schwab, P.; France, M.B.; Ziller, J. W.; Grubbs, R. H.; Angew. Chem., Int. Ed. Engl. 1995, 34, 2039. (b) Schawb, P.; Grubbs, R. H.; Ziller, J. W. J. Am. Chem. Soc. 1996, 118, 100.

140. Kirkland, T. A.; Grubbs, R.H. J. Org. Chem. 1997, 62, 7310.

141. Scholl, M.; Sheng, D.; Lee, C. H.; Grubbs, R. H. Org. Lett. 1999, 1, 953.

142. Dias, E.L.; Nguyen, S. T.; Grubbs, R. H. J. Am. Chem. Soc. 1997, 119, 3887.

143. Louie, J.; Grubbs, R. H. Angew. Chem. Int. Ed. Engl. 2001, 40, 247.

144. Briot, A.; Bujard, M.; Gouverneur, V.; Nolan, S.P.; Mioskowski, C. Org. Lett. $2000,2,1517$.

145. a) Cho., J. H.; Kim, B.M. Org. Lett., 2003, 5, 531; b) Colombier, C.; Fortin, R.; Mortin, H.E.; Skrydstrup, T.; Beau, J.M. Tetrahedron Lett. 1994, 35, 8167; c) 
Gillard, J.W.; Fortin, H.E.; Yoakim, C.; Quesnelle, C.A.; Daignault, S.; Guindon, Y. J. Org. Chem., 1988, 53, 2602; c) Rover, S.; Wipf, P. Tetrahedron Lett. 1999, 40, 5667. d) Bear, B.R.; Parnes, J.S.; Shea, K.J. Org. Lett. 2003, 5, 1613; e) Harrison, B.A.; Gierasch, T.M.; Neilan, C.; Pasternak, G.W.; Verline, G.L. J. Am. Chem. Soc. 2002, 124, 13352; f) Postema, M.H.D.; Piper, J.L., Tetrahedron Lett. 2002, 43, 7095 .

146. See: Hoye, T.R.; Promo, M.A. Tetrahedron Lett. 1999, 40, 1429; for the synthesis of a seven membered (non-substituted) cyclic diphenyl sily tethered ether

147. Xu,Z.; Johannes, C. W.; Houri, A.F.; La, D. S.; Cogan, D.A.; Hofilena, G.E.; Hoveyda, A. H. J. Am. Chem. Soc. 1997, 119, 10302.

148. Grossman, R.B. The art of writing organic reaction mechanisms; Springer; 2003, p 71 .

149. Boiteau, J.G.; Van de Weghe, P.; Eustache, J. Tetrahedron Lett. 2001, 42, 239.

150. Pearson, A.J.; Kim, J.B. Org. Lett. 2002, 4, 2837.

151. Ohno, K.; Nishiyama, H.; Nagase, H. Tetrahedron Lett. 1979, 4405.

152. Khun, R.; Low, I.; Trishmann, H. Chem. Ber. 1957, 90, 203.

153. a) Winder, U. Synthesis 1987,568 ; b) Iversen, T.; Bundle, D.R. J. Chem. Soc. Chem. Commun. 1981, 1240; c) Wessel, H.P.; Iversen, T.; Bundle, D.R. J. Chem. Soc. Perkin Trans. 1. 1985, 2247.

154. Wessel, H.P.; Iversen, T.; Bundle, D.R. J. Chem. Soc. Perkin Trans. 1. 1985, 2247.

155. Maynard, H.D.; Grubbs, R.H. Tetrahedron Lett., 1999, 40, 4137.

156. Kiely, A.F.; Jernelius, J.A.; Schrock, R.R.; Hoveyda, A.H. J. Am. Chem. Soc., 2002, 124, 2686.

157. Paquette, L.A.; Schloss, J.D.; Efremov, I.; Fabris, F.; Gallou, F.; Mendez-Andino, J.; Yang, J. Org. Lett. 2000, 2, 1259.

158. Ahn, Y.M.; Yang, J. Org. Lett. 2001, 3, 1411.

159. Cho., J. H.; Kim, B.M. Org. Lett., 2003, 5, 531.

160. Wakamatsu, H.; Blechert, S. Angew. Chem. Int. Ed.Engl. 2002, 41, 2403.

161. Schürer, S.C.; Gessler, S., Buschmann, N.; Blechert, S. Angew. Chem. Int. Ed.Engl. 2000, 39, 3898.

162. a)Evans, D.A.; Coleman, P.J.; Cote, B. J. Org. Chem. 1997, 62, 788-789; b) Evans, D.A.; Fitch, D.M.; Smith, T.E.; Cee, V.J. J. Am. Chem. Soc. 2000, 122, 
10033; c) Evans, D.A.; Trotter, B.W.; Coleman, P.J.; Cote, B.; Dias, L.C.; Rajapakse, H.A.; Tyler, N. Tetrahedron 1999, 55, 8671.

163. a) Paterson, I.; Gibson, K.R.; Oballa, R.M.; Tetrahedron Lett. 1996, 37, 8585; b) Paterson, I.; Collett, L.A. Tetrahedron Lett. 2001, 42, 1187; c) Paterson, I.; Emilia Di Francesco, M.; Kuhn, T. Org. Lett. 2003, 5, 509; d) Paterson, I.; Oballa, R.M.; Norcross, R.D.; Tetrahedron Lett. 1996, 37, 8581.

164. Paterson, I.; Goodman, J.M.; Lister, M.A.; Schumann, R.C. Tetrahedron, 1990, 46,4663 .

165. Stecher, H.; Faber, K. Synthesis, 1997, 1.

166. For examples of enzymatic resolution of alcohols coupled with rutheniumcatalysed racemisation of the substrate alcohol see a) Larsson, A.L.E.; Persson, B.A.; Bäckvall, J.E. Angew. Chem. Int. Ed. Engl. 1997, 36, 1211; b) Persson, B.A.; Larsson, A.L.E.; Le Ray, M.; Bäckvall, J.E. J. Am. Chem. Soc. 1999, 121, 1645 ; c) Lee, D.; Huh, E.A.; Kim, K.J.; Jung, H.M.; Koh, J.H.; Park, J. Org. Lett. 2000, 2, 2377.

167. For a review on the non-enzymatic kinetic resolution of secondary alcohols see; Somfai, P. Angew. Chem. Int. Ed. Engl. 1997, 36, 2731

168. a) Ferreira, E.M.; Stolz, B.M. J. Am. Chem. Soc. 2001, 123, 7725; b)Mueller, J.A.; Jensen, D.R.; Sigman, M.S. H. J. Am. Chem. Soc. 2002, 124, 8202; c)Jensen, D.R.; Pugsley, J.S.; Sigman, M.S. J. Am. Chem. Soc. 2001, 123, 7475; d) Jensen, D.R.; Sigman, M.S. Org. Lett. 2003, 5, 63; e) Bagdanoff, J.T.; Ferreira, E.M.; Stoltz, B.M. Org. Lett. 2003, 5, 835.

169. Gao, Y.; Klunder, J.M.; Hanson, R.M.; Masammune, H.; Ko, S.Y.; Sharpless, B.K. J. Am. Chem. Soc. 1987, 109, 5765.

170. a) Bellemin-Laponnaz, S.; Tweddell, J.; Ruble, J.C.; Breitling, F.M.; Fu, G.C. Chem. Commun,. 2000, 1009; b) Ruble, J.C.; Lanthan, H.A.; Fu, G.C. J. Am. Chem. Soc. 1997, 119, 1492; c) Ruble, J.C.; Tweddell, J.; Fu, G.C. J. Org. Chem. 1998, 63, 2794.

171. a) Adam, W.; Saha-Möller, C.R.; Schmid, K.S. Tetrahedron Asymm. 1999, 10, 315; b) Gustafsson, J.; Sandström, J.; Sterer, O. Tetrahedron Asymm. 1995, 6, 595; c) Rotticci, D.; Orrenius, C.; hult, K.; Norin, T. Tetrahedron Asymm. 1997, 8,359 .

172. Laumen, K.; Breitgoff, D.; Schneider, M.P. J. Chem. Soc., Chem. Commun. 1988, 1459.

173. Kazlauskas, R.J.; Weissfloch, A.N.E.; Rappaport, A.T.; Cuccia, L.A. J.Org. Chem. 1991, 56, 2656.

174. Yamada, Y.M.A.; Yoshikawa, N.; Sasai, H.; Shibasaki, M. Angew. Chem. Int. Ed. Engl. 1997, 36, 1871. 
175. Trost, B. M.; Silcoff, E. R.; Ito, H. Org. Lett. 2001, 3, 2497.

176. a) List, B.; Lerner, R. A.; Barbas III, C.F.; J. Am. Chem. Soc. 2000, 122, 2395; b) List, B.; Pojarliev, P.; Castello, C. Org. Lett. 2001, 3, 573; c) Notz, W.; List, B. J. Am. Chem. Soc. 2000, 122, 7386.

177. Evans has however reported high 1,5-syn selectivities when using the syn selective chlorophenylboryl enolates; see: a) Evans, D.A.; Calter, M.A.; Tetrahedron Lett. 1993, 34, 6871; b) Evans, D.A.; Fitch, D.M.; J.Org. Chem. 1997, 62, 454.

178. Kozmin, S.A.; Org. Lett. 2001, 3, 755.

179. Jones, G. B.; Chapman, B.J. Synthesis, 1995, 475.

180. Corey, E.J.; Becker, K.B.; Varma, R. K. J. Am. Chem. Soc. 1972, 94, 8616.

181. Binger, P.; Brinkmann, A.; Richter, W. J. Tetrahedron Lett. 1983, 24, 3599.

182. Whitsell, J.K.; Bhattacharya, A.; Henke, K. J. Chem. Soc., Chem. Comm. 1982, 988.

183. Evans, D. A.; Chapman, K.T.; Tan Haung, D.; Kawaguchi, A. T. Angew. Chem. Int. Ed. Engl. 1987, 26, 1184.

184. The twist-boat transition state is based on Houk's and Evan's calculations. See reference 177b and Li, Y.; Padden-Row, M.N.; Houk, K.N. J. Org. Chem. 1990, 55,481 .

185. For an example, see reference 163a.

186. a) Hardinger, S. A.; Wijaya, N. Tetrahedron Lett. 1993, 34, 3821; b) Bhatt, R.K.; Chauhan, K.; Wheelan, P.; Murphy, R. C.; Falck, J. R. J. Am. Chem. Soc. 1994, 116,5050 .

187. Braish, T.F.; Fuchs, P.L. Synth. Communications, 1986, 16, 111.

188. For similar studies on the influence of the aldehyde acceptor on boron mediated 1,3-anti reactions see: Ramachandran, P.V.; Xu, W.C.; Brown, H.C. Tetrahedron Lett. 1996, 37, 4911.

189. Abraham, R.J.; Reid, M.; J. Chem. Soc., Perkin Trans 2, 2002, 1081.

190. Whitesell, J.K.; Younathan, J. N.; Hurst, J.R.; Fox, M. A. J. Org. Chem. 1985, 50, 5499.

191. Nussbaumer, C.; Frater, G. Helv. Chim. Acta, 1987,70, 396.

192. Bhattacharjee, A.; Soltani, O.; De Brabander, J.K. Org. Lett. 2002, 4, 481.

193. Stocker, B.L.; Dong, Y.; Hoberg, J.O.; paper in preparation 
194. The Curtin-Hammlet principle states that product composition, in a reaction which yields one product from one conformational isomer and another product starting with another conformational isomer of the same substrate, does not depend on the population of the ground states but only on the relative energies of the respective transition states. Refer to a) Eleil, E.L. Stereoselectivity of Carbon Compounds, McGrall-Hill, NY, 1962, p 151-152, 237-238 b) Gage, J.R.; Evans, D.A. Org. Synth. 1989,68,77; b) Hammond, G.S. J. Am. Chem. Soc., 1955, 77, 334.

195. Stocker, B.L.; Teesdale-Spittle, P.; Hoberg, J.O. Eur. J. Org. Chem. 2004, 2, 330.

196. Evans, D.A.; Urpi, F.; Somers, T.C.; Clark, J.S.; Bilodeau, M.T. J. Am. Chem. Soc. 1990, 112, 8215; b) Gage, J.R.; Evans, D.A. Org. Synth. 1989,68,77.

197. Brown, H.C.; Dhar, R.K.; Ganesan, K.; Singaram, B. J. Org. Chem. 1992, 57, 499.

198. Hegedus, L.S. Organic Synthesis, vol 79, 2002, pg 103.

199. a) Brown, H.C.; Singaram, B.; J. Org. Chem. 1984, 49, 945; or b) Brown, H.C.; Desai, M.C.; Jadhav, P. K. J. Org. Chem. 1982, 47, 5065.

200. Brown, H,.C.; Chandrasekharan, L.; Ramachandran, P.V. J. Am. Chem. Soc. 1988, 110, 1539.

201. Ciufolini, M. A.; Ning, X. J. Chem. Soc., Chem. Comm.; 1994, 1867.

202. Hall, D.G.; Caillé, A.S.; Drowin, M.; Lamothe, S.; Müller, R.; Deslongchamps, P. Synthesis 1995, 9, 1081 .

203. Paterson, I.; Goodman, J.M.; Lister, M. A.; Schumann, R. C.; McClure, C.K.; Norcross, R.D. Tetrahedron 1990, 46, 4663.

204. Spectral data matched that previously reported; see reference 203.

205. Liao, W.; Wu, Y.; De Brabander, J. K. Angew. Chemie, Int. Ed. Engl. 2003, 42, 1648.

206. Paterson, I.; Di Francesco, M. E.; Kühn, T. Org. Lett. 2003, 5, 599.

207. Gosh, A. K.; Kim, J.-H. Tetrahedron Lett. 2003, 44, 3967

208. Gosh, A. K.; Kim, J.-H. Tetrahedron Lett. 2003, 44, 7659

209. Taylor, R. E.; Jin, M. Org. Lett. 2003, 5, 4959.

210. Engers, D.W.; Bassindale, M.J.; Pagenkopf, B. L.; Org. Lett. 2004, 6, 1057. 